\title{
Understanding Competitive Endogenous RNA Network Mechanism in Type I Diabetes Mellitus Using Computational and Bioinformatics Approaches
}

\author{
Xuanzi $\mathrm{Yi}^{1, *}$ \\ Xu Cheng ${ }^{2,3, *}$ \\ 'Department of Medicine II, Division of \\ Endocrinology and Diabetology, Medical \\ Center University of Freiburg, Faculty of \\ Medicine, University of Freiburg, \\ Freiburg, 79106, Germany; ${ }^{2}$ Department \\ of Urology, Xiangya Hospital, Central \\ South University, Changsha, Hunan, \\ People's Republic of China; ${ }^{3}$ Medical \\ Center University of Freiburg, Faculty of \\ Medicine, University of Freiburg, \\ Freiburg, 79106, Germany \\ *These authors contributed equally to \\ this work
}

Background: Type 1 diabetes mellitus (T1DM), an autoimmune disease with a genetic tendency, has an increasing prevalence. Long non-coding RNA (lncRNA) and circular RNA (circRNA) are receiving increasing attention in disease pathogenesis. However, their roles in T1DM are poorly understood. The present study aimed at identifying signature lncRNAs and circRNAs and investigating their roles in T1DM using the competing endogenous RNA (ceRNA) network analysis.

Methods: The T1DM expression profile was downloaded from Gene Expression Omnibus (GEO) database to identify the differentially expressed circRNAs, lncRNAs, and mRNAs. The biological functions of these differentially expressed circRNAs, IncRNAs, and mRNAs were analyzed by the Gene Ontology (GO) and Kyoto Encyclopedia of Genes and Genomes (KEGG) enrichment analysis. Targeting relationships of circRNA-miRNA, lncRNA-miRNA, and miRNA-mRNA were predicted, and the circRNA-lncRNA-miRNA-mRNA ceRNA regulatory network was established. Finally, qRT-PCR was applied to identify the effect of hsa_circ_0002202 inhibition on the IFN-I induced macrophage inflammation.

Results: A total of 178 circRNAs, 404 lncRNAs, and 73 mRNAs were identified to be abnormally expressed in T1DM samples. Functional enrichment analysis results indicated that the differentially expressed genes were mainly enriched in extracellular matrix components and macrophage activation. CeRNA regulatory network showed that circRNAs and lncRNAs regulate mRNAs through integrate multiple miRNAs. In addition, in vitro experiments showed that hsa_circ_0002202 inhibition suppressed the type I interferon (IFN-I)induced macrophage inflammation.

Conclusion: In the present study, the circRNA-lncRNA-miRNA-mRNA ceRNA regulatory network in T1DM was established for the first time. We also found that hsa_circ_0002202 inhibition suppressed the IFN-I-induced macrophage inflammation. Our study may lay a foundation for future studies on the ceRNA regulatory network in T1DM.

Keywords: type 1 diabetes mellitus, ceRNA network, peripheral blood mononuclear cell, macrophage

\section{Introduction}

Type 1 diabetes mellitus (T1DM), also known as insulin-dependent diabetes mellitus, is a chronic autoimmune disease characterized by impaired islet function and decreased insulin secretion. ${ }^{1,2}$ The cause of T1DM involves various factors, including epigenetic, individual genetics, and environmental triggers. ${ }^{3-6}$ T1DM is diagnosed at all ages. ${ }^{7,8}$ Among them, approximately 78,000 young people are diagnosed with T1DM annually worldwide. ${ }^{9}$ Moreover, recent studies revealed
Department of Medicine II, Division of Endocrinology and Diabetology, Medical Center University of Freiburg, Faculty of Medicine, University of Freiburg, Hugstetter Str. 55, Freiburg, 79106, Germany

Tel/Fax +49 76I 270-73270

Email xuanzi.yi@uniklinik-Freiburg.de 
that the incidence of T1DM is on the rise at the age of 10-14, and the number of children and adolescents with T1DM worldwide is estimated to continue to increase. ${ }^{10,11}$

Patients with T1DM usually suffer from the disease for a longer time, which requires them to inject insulin daily and perform continuous blood glucose monitoring, resulting in huge lifetime costs and time requirements. ${ }^{12}$ At the same time, T1DM has serious complications such as ketoacidosis, heart disease, stroke, kidney failure, and blindness. ${ }^{13-17}$ These long-term effects are likely to spread to other areas of the patients' lives, which will cause economic impact and social burdens. ${ }^{12,18}$ Thus, it is necessary to diagnose T1DM early. However, the traditional diagnostic methods of diabetes are no longer satisfactory. ${ }^{19}$ Therefore, it is urgent to understand the etiology and pathogenesis of T1DM for more effective diagnosis and treatment.

Through the recent researches on the post-transcriptional regulatory mechanism, non-coding RNA (ncRNA), which does not have traditional RNA functions in protein translation, was discovered. ${ }^{20}$ Among them, the long noncoding RNA (lncRNA) is a kind of linear non-coding RNA with a length of more than 200 nucleotides, ${ }^{21}$ and circular RNA (circRNA) is a kind of endogenous noncoding RNA with a closed-loop structure. ${ }^{22}$ Previous studies have shown the vital roles of various lncRNAs and circRNAs in T1DM. IncRNA Lnc13 is up-regulated in $\beta$ cells and contributes to the pathogenesis of T1DM by increasing pancreatic $\beta$-cell inflammation. ${ }^{23}$ Ding et al reported that IncRNA MALAT1 induces the dysfunction of $\beta$-cells in T1DM. ${ }^{24}$ Another study by Zhang et al found that circRNA circPPM1F modulates M1 macrophage activation and pancreatic islet inflammation in $\mathrm{T}_{1 \mathrm{DM}}{ }^{25}$ Therefore, examining the expression of lncRNAs and circRNAs and exploring their underlying mechanism in T1DM opens avenues to a better understanding of the T1DM pathogenesis.

Both IncRNA and circRNA can act as microRNA (miRNA) sponges to compete with the same corresponding miRNA response element (MRE), thus controlling subsequent miRNA post-transcriptional regulation and forming competitive endogenous RNA (ceRNA) regulatory network. ${ }^{26-28}$ The ceRNA regulatory networks in diabetes have been widely reported. ${ }^{29-32}$ While in T1DM, few studies regarding the ceRNA regulatory network were reported. $\mathrm{Li}$ et al and Luo et al identified the circRNA-miRNA-mRNA network in T1DM via bioinformatic analysis. ${ }^{22,33}$ Shi et al reported the lncRNA-miRNA-
mRNA regulatory networks in T1DM. ${ }^{34}$ Thus, further studies aimed at clarifying ceRNA-based molecular mechanisms in T1DM are needed to provide potential opportunities for better understanding the pathogenesis and treatment of T1DM.

With the popularization of high-throughput sequencing, bioinformatics analysis has been widely used in multiple disease research. Researchers can analyze data from various public databases and explore the regulatory mechanism underlying the disease. Here, we identified the differentially expressed circRNAs (DEcircRNAs), lncRNAs (DElncRNAs), mRNAs (DEmRNAs) in T1DM using expression profile GSE133225 and GSE133217 downloaded from the Gene Expression Omnibus (GEO) database. ${ }^{35}$ Subsequently, Gene Ontology (GO) and Kyoto Encyclopedia of Genes and Genomes (KEGG) enrichment analyses were performed to detect the function of these differentially expressed genes. Then, the circRNAmiRNA, IncRNA-miRNA, and miRNA-mRNA interaction were predicted, and the circRNA-IncRNA-miRNA-mRNA ceRNA regulatory network was constructed. This is the first time to construct the ceRNA regulatory network by comprehensive screening of circRNA, lncRNA, miRNA, and mRNA in T1DM.

\section{Materials and Methods Data Sources}

Two individual cohorts from the GEO database (http:// www.ncbi.nlm.nih.gov/geo/) were included in this study. One is the circRNA, IncRNA, and mRNA expression profile GSE133225, and the other one is the miRNA expression profile GSE133217. Both expression profiles were provided by Zhang Caiyan and Zhou Yufeng, ${ }^{25,36}$ and included 4 peripheral blood mononuclear cells (PBMCs) samples from T1DM patients and 4 PBMCs samples from healthy volunteers.

\section{Summary of Statistical Analysis}

In the current study, we first analyzed the circRNA, IncRNA, miRNA, and mRNA expression using the microarray GSE133225 and GSE133217. Next, according to the filtrate threshold $(P$ value $<0.05$ and $\log 2$ (fold change) $>$ $1)$, the differentially expressed circRNA, lncRNA, and mRNA were screened out. GO and KEGG enrichment analyses were performed to better comprehend the mechanisms of T1DM. Then, the target prediction was performed with the online web tools Circinteractome, 
LncBase, and starBase, and the circRNA-lncRNAmiRNA-mRNA interaction network was established. Finally, circRNA hsa_circ_0002202 was selected for further verification. A brief workflow was shown in Figure 1.

\section{Differential Expression Analysis}

R software (Ver. 3.5.0, https://www.r-project.org/) was employed for microarray analysis. The "affy" package was used for normalizing microarray expression profiles as described before. ${ }^{37}$ Then, empirical Bayes moderated $t$-test in the "limma" package ${ }^{38}$ was applied to screen the differentially expressed genes (DEGs) between T1DM samples and normal samples with $P$ value $<0.05$ and $\log 2$ (fold change) $>1$ as the threshold. The code for the differential expression analysis was shown in $\underline{\text { Supplementary File } 1 .}$

\section{Functional and Pathway Enrichment Analysis of Differentially Expressed circRNAs, IncRNAs, and mRNAs}

To better comprehend the mechanisms of T1DM, GO (http://www.geneontology.org/) analysis and KEGG (http://www.kegg.jp/) pathway analysis of the differentially expressed circRNA, IncRNA, and mRNAs was

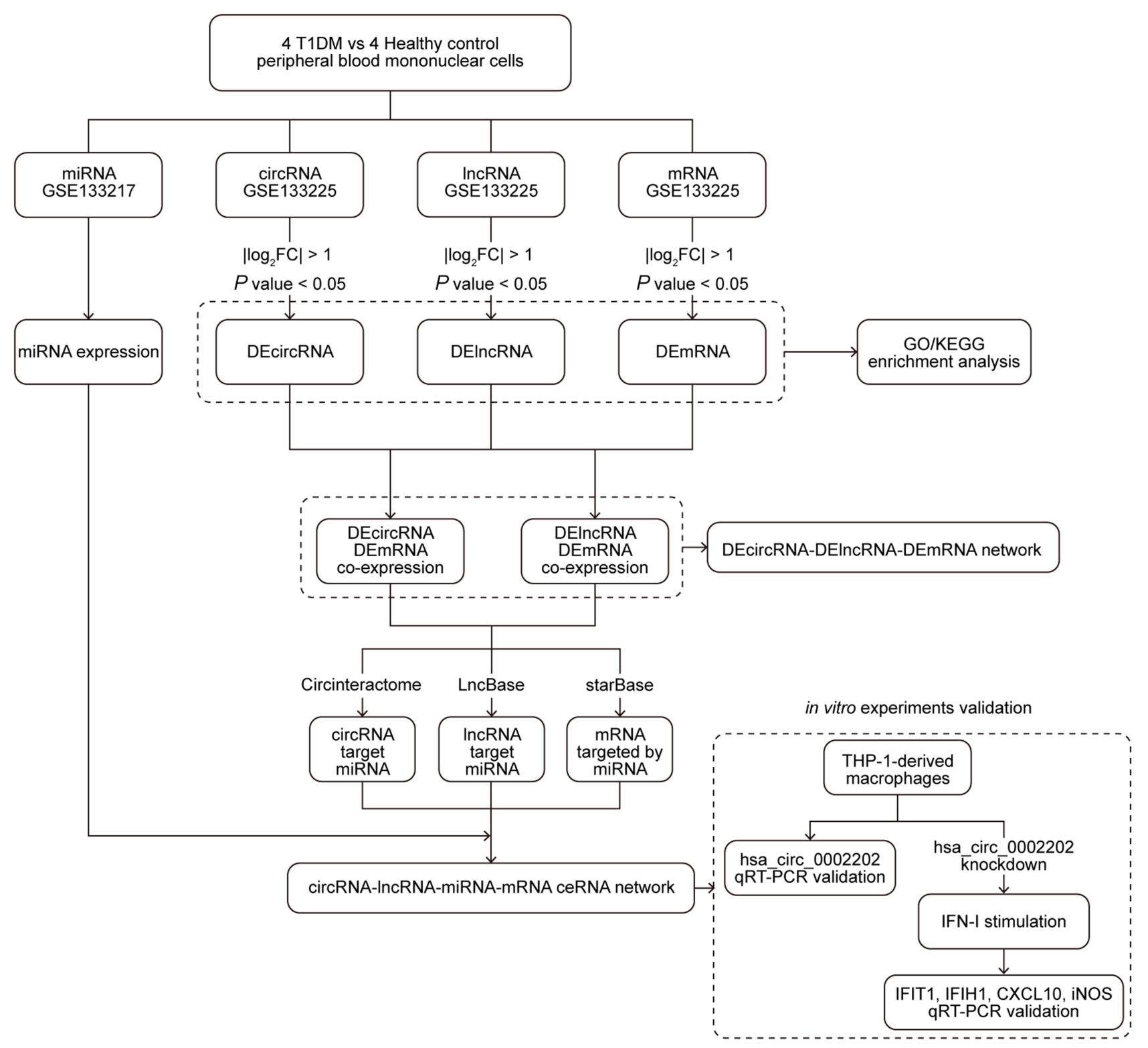

Figure I The analysis flow chart of this study. 
conducted with the "clusterProfiler" package ${ }^{39}$ using $P<$ 0.05 (adjusted using Benjamin and Hochberg method) as the threshold. Specifically, GO analysis is used to construct gene annotation base on biological processes (BP, a biological objective to which the gene or gene product contributes), cellular components (CC, the place in the cell where a gene product is active), and molecular functions (MF, the biochemical activity (including specific binding to ligands or structures) of a gene product), ${ }^{40}$ and KEGG analysis is used to interpret the potential functions and pathways. ${ }^{41}$ The code for the enrichment analysis was shown in Supplementary File 2. For the enrichment analysis for differentially expressed circRNAs, we used their parental genes. For the enrichment analysis for differentially expressed lncRNAs, we used their associated genes.

\section{Construction of circRNA-IncRNA- miRNA-mRNA ceRNA Regulatory} Network

The expression correlation of differentially expressed circRNAs, lncRNAs, and mRNAs was calculated using Pearson correlation coefficients with the "psych" package (https://cran.r-project.org/web/packages/psych/). The code for the correlation analysis was shown in Supplementary File 3. circRNA-mRNA and lncRNA-mRNA with $r>0.7$ and $P<0.05$ were selected for further study. Then, the Circinteractome web tool (https://circinteractome.nia.nih. gov/) was applied to predict the circRNA-miRNA interactions. LncBase web tool (http://carolina.imis.athena-inno vation.gr/diana_tools/web/index.php?r=lncbasev2/index) was applied to predict the lncRNA-miRNA interactions. starBase web tool (http://starbase.sysu.edu.cn/) was applied to predict the miRNA-mRNA interactions. Finally, a circRNA-lncRNA-miRNA-mRNA interaction network visualized by Cytoscape software ${ }^{42}$ based on the ceRNA theory.

\section{Cell Culture and Treatment}

Human THP-1 cell line from Procell Life Science\&Technology Co., Ltd. (Wuhan, China) was cultured in the RPMI-1640 medium supplied with 10\% fetal bovine serum (FBS, Gibco, Gaithersburg, MD, USA) and $1 \%$ penicillin-streptomycin (Gibco) at $37^{\circ} \mathrm{C}$ with $5 \% \mathrm{CO}_{2}$. Then, according to a previous study, ${ }^{36}$ THP-1-derived macrophages were induced by treating with $50 \mathrm{ng} / \mathrm{mL}$ phorbol 12-myristate 13-acetate (PMA) for $48 \mathrm{~h}$. Cell transfection was performed using Lipofectamine 3000 reagent (Invitrogen, USA) with the chemically synthesized circRNA small interfering RNAs (si-circ, sequence: 5'AGTACAACAATTCAGGTGATT-3'), and small interfering RNAs negative control (si-NC, sequence: 5'UUCUCCGAACGUGUCACGUTT-3'). After $48 \mathrm{~h}$ of transfection, the macrophages were treated with type I interferon (IFN-I, 1000 units/mL, PBL Assay Science, USA) to induce the inflammation.

\section{Quantitative Real-Time PCR (qRT-PCR)}

Total RNA was extracted from cells with Trizol (Invitrogen, USA). Next, cDNA was reverse transcribed with PrimeScript First Strand cDNA Synthesis Kit (Takara, Japan). Then, the qRT-PCR reaction was performed with SYBR ${ }^{\circledR}$ Premix Ex Taq ${ }^{\mathrm{TM}}$ II (Takara, Japan) on the LightCycler 96 PCR system (Roche, Rotkreuz, Switzerland). $\beta$-actin was selected as the internal control according to the geNorm ${ }^{43}$ and normFinder ${ }^{44}$ algorithms. The level of RNA was quantified by the $2^{-\Delta \Delta C T}$ method. Primers were displayed in Table 1.

\section{Nuclear and Cytoplasmic Separation}

In brief, nuclear and cytoplasmic RNAs were extracted using the PARIS kit (Invitrogen, Carlsbad, CA, USA). Then, the RNA extractions were subjected to qRT-PCR.

Table I Primers Used for qRT-PCR

\begin{tabular}{|l|l|}
\hline Name & Sequence (5'-3') \\
\hline IFITI forward & GCCTTGCTGAAGTGTGGAGGAA \\
IFITI reverse & ATCCAGGCGATAGGCAGAGATC \\
IFIHI forward & GACTCGGGAATTCGTGGAGG \\
IFIHI reverse & CTCAAACGATGGAGAGGGCA \\
iNOS forward & GCTCTACACCTCCAATGTGACC \\
iNOS reverse & CTGCCGAGATTTGAGCCTCATG \\
CXCLI0 forward & GGTGAGAAGAGATGTCTGAATCC \\
CXCLI0 reverse & GTCCATCCTTGGAAGCACTGCA \\
hsa_circ_0002202 forward & GACAAAAGCATGGGGTGTGAG \\
hsa_circ_0002202 reverse & GCCTCTTCCCATCACCTGAAT \\
GAPDH forward & GTCTCCTCTGACTTCAACAGCG \\
GAPDH reverse & ACCACCCTGTTGCTGTAGCCAA \\
I8S rRNA forward & ACACGGACAGGATTGACAGA \\
I8S rRNA reverse & GGACATCTAAGGGCATCACA \\
$\beta$-actin forward & CACCATTGGCAATGAGCGGTTC \\
$\beta$-actin reverse & AGGTCTTTGCGGATGTCCACGT \\
$\beta$-tubulin forward & TGGACTCTGTTCGCTCAGGT \\
$\beta$-tubulin reverse & TGCCTCCTTCCGTACCACAT \\
U6 forward & CTCGCTTCGGCAGCACA \\
U6 reverse & AACGCTTCACGAATTTGCGT \\
\hline
\end{tabular}


U6 was used as the internal control of the nucleus, and $18 \mathrm{~S}$ rRNA was used as the internal control of the cytoplasm.

\section{Statistical Analysis}

The qRT-PCR experiments were performed in triplicate, and the results were shown as mean \pm SD. Statistical difference was analyzed using one-way ANOVA with GraphPad Prism (Ver. 8.0, GraphPad Software, Inc). $P<$ 0.05 was considered to be statistically significant.

\section{Results}

\section{Identification of Differentially Expressed circRNA in TIDM and Healthy Patients}

By analyzing the circRNA expression in microarray GSE133225 (Figure 2A and B), we identified 178 differentially expressed circRNAs (DEcircRNAs, 77 up-regulated and 101 down-regulated) (Table 2). We also analyzed the chromosomal positions of these DEcircRNAs, and the top 5 chromosomes are as follows: $\operatorname{chr} 1(18,10.11 \%)$, chr2 $(15,8.43 \%)$, chr3 (12, 6.74\%), chr7 $(12,6.74) \%)$, chr10 (11, 6.18\%). Functional enrichment analysis of DEcircRNAs was performed using the "clusterProfiler" tool. The results showed that the most significantly enriched GO terms were related to collagen fibril organization (GO: 0030199), membrane raft (GO: 0045121), and extracellular matrix structural constituent conferring tensile strength (GO: 0030020) (Figure $2 \mathrm{C}$ and Table 3). For the KEGG pathway analysis, the DEcircRNAs were enriched in protein digestion and absorption (hsa04974) (Table 4).

\section{Identification of Differentially Expressed IncRNA in TIDM and Healthy Patients}

Similarly, we analyzed the lncRNA expression with the microarray GSE133225. As shown in the volcano plot (Figure 3A) and heatmap (Figure 3B), 404 differentially expressed lncRNAs were screened (DElncRNAs, 199 up-regulated and 205 down-regulated) (Table 5). Meanwhile, the top 5 chromosomal positions of these DElncRNAs are as follows: chr1 $(34,8.42 \%), \operatorname{chr} 2(29$, 7.18\%), chr17 (25, 6.19\%), chr3 (25, 6.19\%), chr10 (24, $5.94 \%$ ). The GO enrichment analysis results showed that the DElncRNAs were enriched in macrophage activation (GO:0042116) and microvillus (GO:0005902) (Figure $3 \mathrm{C}$ and Table 6).
Identification of Differentially Expressed mRNA in TIDM and Healthy Patients

Subsequently, the differentially expressed mRNAs between the normal and T1DM groups were analyzed. As the volcano plot (Figure 4A) and heatmap (Figure 4B) indicated, there were 73 differentially expressed mRNAs (DEmRNAs, 31 up-regulated and 42 down-regulated) (Table 7). Most of them were located at chr2 $(7,9.59 \%), \operatorname{chr} 7(6,8.22 \%), \operatorname{chr} 17(5$, $6.85 \%), \operatorname{chr} 20(5,6.85 \%), \operatorname{chr} 12(4,5.48 \%)$. In addition, GO enrichment analysis showed that the DEmRNAs were enriched in prostaglandin receptor activity (GO:0004955), prostanoid receptor activity (GO:0004954), and BMP binding (GO:0036122) (Figure 4C and Table 8).

\section{The circRNA-IncRNA-miRNA-mRNA Network in TIDM}

Due to the large number of differentially expressed circRNAs and IncRNAs, we further analyzed their correlation with the DEmRNAs expression and constructed the circRNA-lncRNA-mRNA network using $r>0.7$ as the screening criteria (Figure 5). Meanwhile, according to the ceRNA hypothesis, IncRNA and circRNA can function as miRNA sponges that bind to miRNA and inhibit the regulation of miRNAs on its target mRNAs, thereby indirectly regulating gene expression. Thus, we predicted the miRNAs targeting the DEcircRNAs and DElncRNAs and analyzed their expression using the dataset GSE133217 (Table 9). After screening, the circRNA-lncRNA-miRNA-mRNA network was visualized using Cytoscape software (Figure 6). For example, hsa_circ_0002202 was identified as ceRNA of miR-4953p, miR-668-3p, miR-508-3p, miR-487a-3p, which targeted GREM2. IncRNA LINC01007 regulated HSPB8 by competing for miR-33b-5p, miR-1321, and miR455-3p.

\section{Hsa_circ_0002202 Inhibition Suppressed the IFN-I-Induced Inflammation}

To confirm our above discovery and conceive future study designs, we chose hsa_circ_0002202 with more miRNA targets for in vitro experiments. We first used the geNorm and normFinder algorithms to select the reference gene for normalization. As shown in Supplementary Figure 1, $\beta$-actin was the most stable reference gene. Then, subcellular distribution analysis 
A

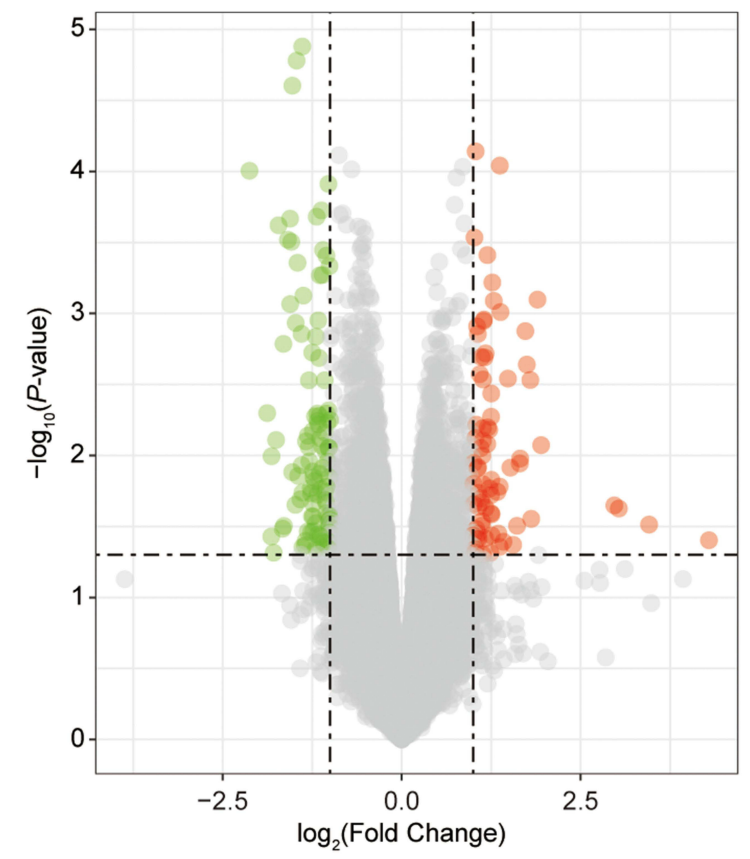

B

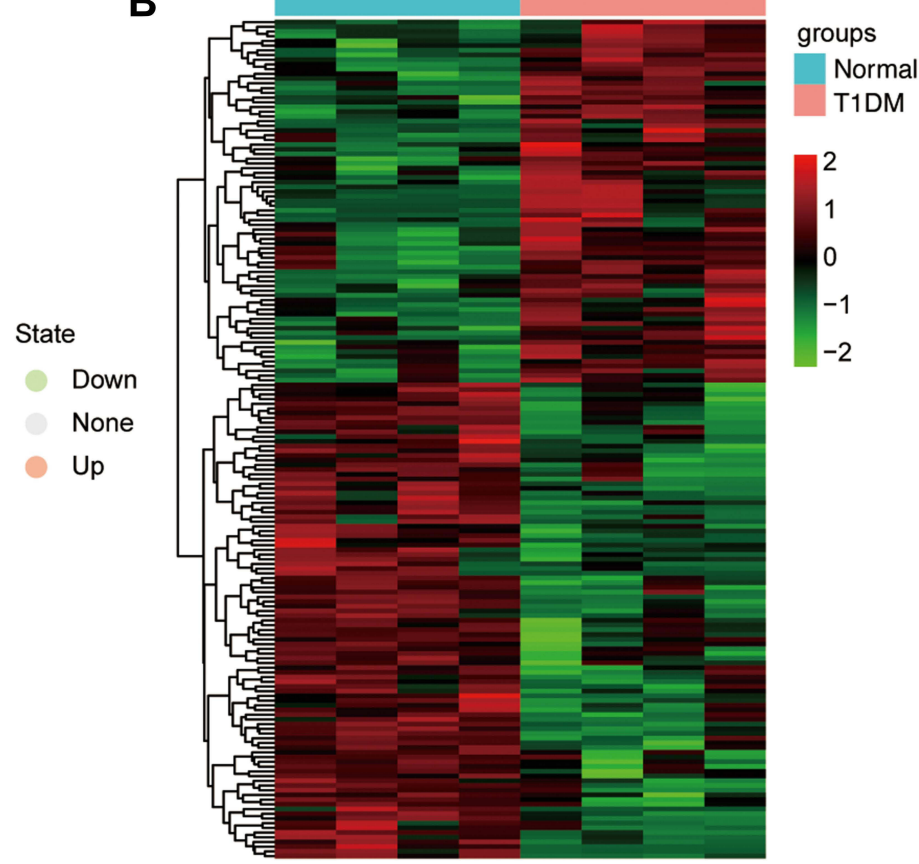

C

Enriched GO Terms

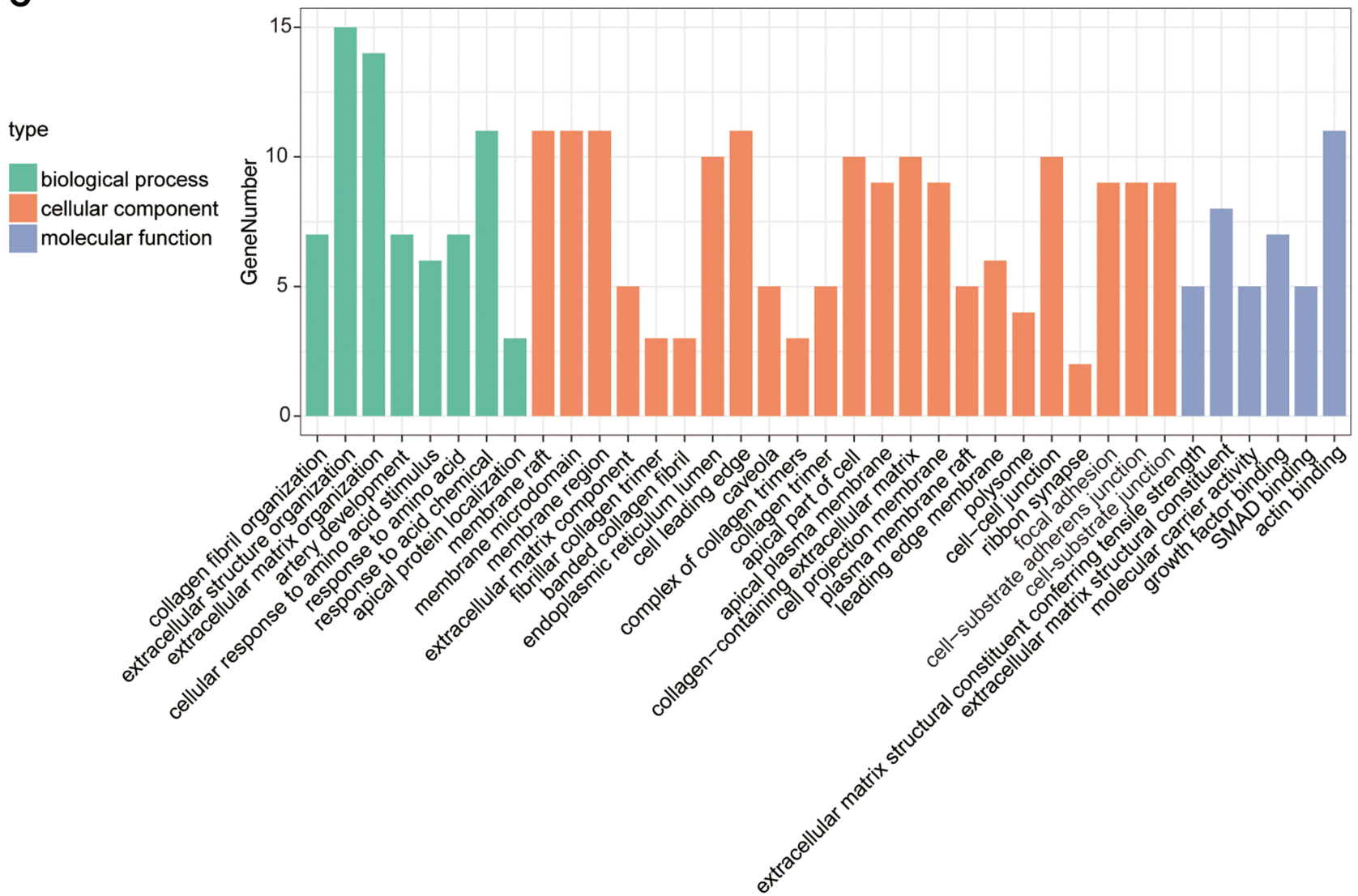

Figure 2 The differentially expressed circRNAs in TIDM and healthy patients. (A) Volcano plot of DEcircRNAs between normal and TIDM groups. Red and green indicate up- and downregulation, respectively. (B) Heatmap of DEcircRNAs between normal and TIDM groups. Red color represents increased expression, and green color represents decreased expression. The darker the color, the greater the difference of circRNA expression. (C) Enrichment analysis of the DEcircRNAs in the categories biological process, cellular component, and molecular function. 
Table 2 Differentially Expressed circRNAs Between the Control Group and TIDM Group

\begin{tabular}{|c|c|c|c|c|}
\hline circRNAs & Log2FoldChange & Regulation & P-value & P-adj \\
\hline hsa_circ_0060875 & -2.1258337 & Down & $9.90 \times 10^{-5}$ & 0.36011603 \\
\hline hsa_circ_009II 20 & -1.8790129 & Down & 0.00502998 & 0.61872814 \\
\hline hsa_circ_0018219 & -1.8216577 & Down & 0.03718813 & 0.6896574 \\
\hline hsa_circ_0083266 & -1.8178634 & Down & 0.01012685 & 0.6404092 \\
\hline hsa_circ_008I949 & $-|.787727|$ & Down & 0.04837352 & 0.69864211 \\
\hline hsa_circ_00I7702 & $-1.75444 \mid 7$ & Down & 0.00777686 & $0.637 \mid 225$ \\
\hline hsa_circ_00898I7 & -1.7206724 & Down & $0.0002394 \mid$ & 0.36707996 \\
\hline hsa_circ_006I76I & -1.6617103 & Down & 0.03278591 & 0.6886992 \\
\hline hsa_circ_0043949 & -1.6515359 & Down & 0.00163728 & 0.49940512 \\
\hline hsa_circ_0026805 & -1.6448912 & Down & 0.03133761 & 0.6886992 \\
\hline hsa_circ_0066856 & -1.5895376 & Down & 0.00030271 & 0.36707996 \\
\hline hsa_circ_0010600 & $-|.558822|$ & Down & 0.00021508 & 0.36707996 \\
\hline hsa_circ_0089863 & -1.5576906 & Down & 0.00085911 & 0.45375667 \\
\hline hsa_circ_00I 2945 & -1.5426338 & Down & 0.00031327 & 0.36707996 \\
\hline hsa_circ_0087II 8 & -1.5293394 & Down & 0.01309075 & 0.6461669 \\
\hline hsa_circ_0025477 & -1.5269283 & Down & $2.49 \times 10^{-5}$ & 0.27197545 \\
\hline hsa_circ_0035318 & $-1.484608 \mid$ & Down & 0.02228095 & 0.67318317 \\
\hline hsa_circ_00 II 345 & -1.4808367 & Down & 0.00116665 & 0.47332912 \\
\hline hsa_circ_0029046 & -1.4680823 & Down & $1.66 \times 10^{-5}$ & 0.27136735 \\
\hline hsa_circ_0070052 & -1.4551817 & Down & 0.00043961 & 0.37883821 \\
\hline hsa_circ_0055707 & $-1.446 \mid 302$ & Down & 0.01391048 & 0.6461669 \\
\hline hsa_circ_00I8927 & -1.4141948 & Down & 0.0203531 & 0.66888734 \\
\hline hsa_circ_0053289 & $-1.399247 \mid$ & Down & 0.00139236 & 0.49940512 \\
\hline hsa_circ_00557/3 & $-1.396499 \mid$ & Down & 0.01169841 & 0.6461669 \\
\hline hsa_circ_0092322 & -1.3886845 & Down & $1.32 \times 10^{-5}$ & $0.27 \mid 36735$ \\
\hline hsa_circ_0045848 & -1.3854297 & Down & 0.04413029 & 0.69594076 \\
\hline hsa_circ_0069584 & -1.3853501 & Down & 0.01824692 & $0.6566285 I$ \\
\hline hsa_circ_000844I & -1.3733694 & Down & 0.00074912 & 0.44105722 \\
\hline hsa_circ_0073007 & $-1.35 \mid 8705$ & Down & 0.04300145 & 0.69594076 \\
\hline hsa_circ_0030977 & -1.3421675 & Down & 0.03991266 & 0.69072451 \\
\hline hsa_circ_0088254 & -1.3367378 & Down & 0.00795582 & 0.63723776 \\
\hline hsa_circ_00I 2093 & $-|.3| 272 \mid$ & Down & 0.03432713 & 0.6886992 \\
\hline hsa_circ_0034676 & -1.3119179 & Down & 0.0072544 & $0.637 \mid 225$ \\
\hline hsa_circ_0082293 & -1.2977776 & Down & 0.00872193 & 0.63723776 \\
\hline hsa_circ_0080325 & $-|.29575| \mid$ & Down & 0.0029555 & 0.52887377 \\
\hline hsa_circ_00047/2 & -1.2890482 & Down & 0.01102659 & 0.6461669 \\
\hline hsa_circ_006838I & -1.270335 & Down & 0.01699615 & 0.65626057 \\
\hline hsa_circ_0042502 & $-1.265 \mid 427$ & Down & 0.03467318 & 0.6886992 \\
\hline hsa_circ_00254I 3 & -1.2635234 & Down & 0.0184742 & 0.6573827 \\
\hline hsa_circ_0068488 & -1.2544915 & Down & 0.02684532 & 0.6886992 \\
\hline hsa_circ_0072670 & -1.24989 & Down & 0.00188517 & $0.52606 / 24$ \\
\hline hsa_circ_0078706 & -1.2409986 & Down & 0.02179647 & 0.67318317 \\
\hline hsa_circ_0025473 & -1.2401025 & Down & 0.00671056 & $0.637 \mid 225$ \\
\hline hsa_circ_0080593 & -1.2388893 & Down & 0.0129599 & 0.6461669 \\
\hline hsa_circ_0082426 & -1.236374 & Down & 0.02682174 & 0.6886992 \\
\hline hsa_circ_0002456 & -1.2308499 & Down & 0.0340347 & 0.6886992 \\
\hline hsa_circ_0035624 & -1.229398 & Down & 0.01432306 & 0.6461669 \\
\hline hsa_circ_0027593 & -1.2143428 & Down & 0.01510762 & 0.6461669 \\
\hline hsa_circ_0033522 & -1.2109569 & Down & 0.00530799 & 0.61994831 \\
\hline hsa_circ_00708I3 & -1.2004595 & Down & 0.04540634 & 0.69594076 \\
\hline hsa_circ_0089089 & -1.197761 & Down & 0.03000448 & 0.6886992 \\
\hline
\end{tabular}

(Continued) 
Table 2 (Continued).

\begin{tabular}{|c|c|c|c|c|}
\hline circRNAs & Log2FoldChange & Regulation & P-value & P-adj \\
\hline hsa_circ_0008732 & -1.1974429 & Down & 0.0014563 & 0.49940512 \\
\hline hsa_circ_0083243 & $-1.1873 \mid 78$ & Down & 0.00020845 & 0.36707996 \\
\hline hsa_circ_0044570 & -1.1776689 & Down & 0.00506876 & 0.61872814 \\
\hline hsa_circ_005I 957 & $-1.176653 \mid$ & Down & 0.0188451 & 0.66175152 \\
\hline hsa_circ_0007872 & -1.1753034 & Down & 0.00571746 & 0.6277625 \\
\hline hsa_circ_0003 | 46 & -1.1698955 & Down & 0.03610804 & 0.6896574 \\
\hline hsa_circ_0088267 & -1.1654894 & Down & 0.0060454 & 0.62927103 \\
\hline hsa_circ_0020296 & -1.1650009 & Down & 0.00111814 & 0.47332912 \\
\hline hsa_circ_00I 2285 & -1.153328 & Down & 0.00205924 & 0.52606124 \\
\hline hsa_circ_0086765 & -1.1459122 & Down & 0.00525476 & 0.61994831 \\
\hline hsa_circ_008464l & -1.1433833 & Down & 0.01349551 & 0.6461669 \\
\hline hsa_circ_00I5060 & -1.1429878 & Down & $0.0005387 \mid$ & 0.41025776 \\
\hline hsa_circ_0065I73 & -1.1388487 & Down & 0.00755684 & $0.637 \mid 225$ \\
\hline hsa_circ_0043616 & -1.1332645 & Down & 0.03696064 & 0.6896574 \\
\hline hsa_circ_0047903 & -1.1276276 & Down & 0.01716979 & 0.65626057 \\
\hline hsa_circ_0018827 & -1.1202055 & Down & 0.00018818 & 0.36707996 \\
\hline hsa_circ_00I5069 & -1.1175352 & Down & 0.04417262 & 0.69594076 \\
\hline hsa_circ_00328I3 & -1.1131832 & Down & $0.0085554 I$ & 0.63723776 \\
\hline hsa_circ_0019143 & -1.112285 & Down & 0.04029593 & $0.6907245 I$ \\
\hline hsa_circ_0008537 & -1.1053958 & Down & 0.00053611 & 0.41025776 \\
\hline hsa_circ_0002529 & $-1.104834 \mid$ & Down & 0.01225173 & 0.6461669 \\
\hline hsa_circ_0046840 & -1.0988722 & Down & 0.00035777 & 0.36707996 \\
\hline hsa_circ_0028284 & -1.0905105 & Down & $0.04 I 20974$ & 0.69432323 \\
\hline hsa_circ_0019432 & -1.0752782 & Down & 0.00295458 & 0.52887377 \\
\hline hsa_circ_0047303 & -1.0705012 & Down & 0.00689965 & $0.637 \mid 225$ \\
\hline hsa_circ_0072I 37 & -1.0684678 & Down & 0.01800286 & 0.65626057 \\
\hline hsa_circ_0024I 30 & -1.0663774 & Down & 0.0205363 & 0.6691564 \\
\hline hsa_circ_0022919 & -1.0578978 & Down & 0.0093951 & 0.63723776 \\
\hline hsa_circ_0022238 & -1.0545557 & Down & 0.02150931 & 0.67318317 \\
\hline hsa_circ_0086805 & -1.0531799 & Down & 0.01662828 & $0.6547 \mid 981$ \\
\hline hsa_circ_0077069 & -1.0513113 & Down & 0.02550238 & 0.68798091 \\
\hline hsa_circ_0057374 & -1.0509624 & Down & 0.00039234 & 0.36707996 \\
\hline hsa_circ_006932I & $-1.049 \mid 406$ & Down & 0.00507185 & 0.61872814 \\
\hline hsa_circ_000747I & -1.0383975 & Down & 0.01103246 & 0.6461669 \\
\hline hsa_circ_0032858 & -1.0382432 & Down & $0.005883 \mathrm{II}$ & 0.62927103 \\
\hline hsa_circ_0033892 & -1.0300829 & Down & 0.04042024 & 0.69072451 \\
\hline hsa_circ_0024575 & -1.023773 & Down & 0.04526006 & 0.69594076 \\
\hline hsa_circ_0083836 & -1.0232697 & Down & 0.00482139 & 0.60677969 \\
\hline hsa_circ_0064419 & -1.0230977 & Down & 0.00012256 & 0.3648499 \\
\hline hsa_circ_0030657 & -1.0226422 & Down & 0.00883236 & 0.63723776 \\
\hline hsa_circ_0087729 & -1.0196745 & Down & 0.03005932 & 0.6886992 \\
\hline hsa_circ_0020964 & -1.0153533 & Down & 0.02651006 & 0.6886992 \\
\hline hsa_circ_0078325 & -1.0146273 & Down & 0.0142381 & 0.6461669 \\
\hline hsa_circ_0068030 & -1.0135546 & Down & 0.00881102 & 0.63723776 \\
\hline hsa_circ_0087884 & -1.0107733 & Down & $0.0287400 \mathrm{I}$ & 0.6886992 \\
\hline hsa_circ_0067/27 & -1.0102228 & Down & 0.03491637 & 0.6886992 \\
\hline hsa_circ_0077292 & $-1.009844 \mid$ & Down & 0.03166391 & 0.6886992 \\
\hline hsa_circ_0050829 & -1.0079665 & Down & 0.00046365 & 0.38552675 \\
\hline hsa_circ_0088545 & $-|.0038| 4 \mid$ & Down & 0.02816448 & 0.6886992 \\
\hline hsa_circ_0066559 & -1.0001944 & Down & 0.00562765 & 0.62317278 \\
\hline
\end{tabular}

(Continued) 
Table 2 (Continued).

\begin{tabular}{|c|c|c|c|c|}
\hline circRNAs & Log2FoldChange & Regulation & P-value & P-adj \\
\hline hsa_circ_003069l & 1.00075362 & Up & 0.0365078 I & 0.6896574 \\
\hline hsa_circ_0073355 & 1.00406779 & Up & 0.01584992 & 0.65471981 \\
\hline hsa_circ_0060973 & 1.00552219 & Up & 0.01129814 & 0.6461669 \\
\hline hsa_circ_007|490 & 1.00837299 & Up & 0.02198034 & 0.67318317 \\
\hline hsa_circ_0073332 & 1.01653192 & Up & 0.00029189 & 0.36707996 \\
\hline hsa_circ_004I 267 & 1.02731163 & Up & 0.04826044 & 0.69864211 \\
\hline hsa_circ_0034557 & I.02903097 & $U_{p}$ & 0.04566388 & 0.69594076 \\
\hline hsa_circ_00I4206 & 1.03151729 & Up & 0.00754327 & $0.637 I 225$ \\
\hline hsa_circ_0034188 & 1.03292459 & Up & $7.23 \times 10^{-5}$ & 0.36011603 \\
\hline hsa_circ_0084862 & 1.04447426 & Up & 0.03297313 & 0.6886992 \\
\hline hsa_circ_0043575 & $1.0445 \mid 466$ & Up & 0.00606669 & 0.62927103 \\
\hline hsa_circ_0066752 & 1.05303312 & Up & 0.00123282 & 0.48639953 \\
\hline hsa_circ_0018918 & 1.05369965 & Up & 0.03867368 & 0.6896574 \\
\hline hsa_circ_0030816 & 1.06017092 & Up & 0.01231415 & 0.6461669 \\
\hline hsa_circ_0002308 & 1.06451001 & Up & 0.00139462 & 0.49940512 \\
\hline hsa_circ_0057940 & 1.06723667 & Up & 0.0355786 & 0.6886992 \\
\hline hsa_circ_0084763 & 1.06852044 & Up & 0.0121094 & 0.6461669 \\
\hline hsa_circ_0079554 & I.06923574 & Up & 0.02296689 & 0.67670006 \\
\hline hsa_circ_0003310 & I.07580527 & Up & 0.03868275 & 0.6896574 \\
\hline hsa_circ_0072445 & 1.0780832 & Up & 0.01833869 & 0.6573827 \\
\hline hsa_circ_0004607 & 1.09052865 & Up & 0.0026797 & 0.52887377 \\
\hline hsa_circ_0074026 & I. 10437363 & Up & 0.00887386 & 0.63723776 \\
\hline hsa_circ_00I 3280 & I.II294406 & Up & 0.02142065 & 0.67318317 \\
\hline hsa_circ_0000324 & I.II328742 & Up & 0.02004466 & 0.6663805 \\
\hline hsa_circ_00I I 437 & 1.11571417 & Up & 0.00203065 & 0.52606124 \\
\hline hsa_circ_0038I62 & I.12089009 & Up & $0.03|203|$ & 0.6886992 \\
\hline hsa_circ_000656I & 1.12140856 & Up & 0.02905904 & 0.6886992 \\
\hline hsa_circ_001932I & I. 12534202 & Up & 0.01003317 & 0.6404092 \\
\hline hsa_circ_0066588 & I.13259964 & Up & $0.00644 \mid 24$ & $0.637 I 225$ \\
\hline hsa_circ_0068846 & I.13350867 & Up & 0.04980974 & 0.69965823 \\
\hline hsa_circ_004894I & I.13392642 & Up & 0.00783921 & $0.637 I 225$ \\
\hline hsa_circ_00I 3276 & 1.1353225 & Up & 0.01594875 & $0.6547|98|$ \\
\hline hsa_circ_0084803 & 1.13561704 & Up & 0.00291642 & 0.52887377 \\
\hline hsa_circ_00097/8 & I.1450I876 & Up & 0.00113062 & 0.47332912 \\
\hline hsa_circ_0052890 & I.14899657 & Up & 0.00110269 & 0.47332912 \\
\hline hsa_circ_0052578 & I.1574625I & Up & 0.00204063 & $0.52606 \mid 24$ \\
\hline hsa_circ_0004247 & 1.16577189 & Up & 0.03834653 & 0.6896574 \\
\hline hsa_circ_0006366 & I.1706086| & Up & 0.02336843 & 0.67886043 \\
\hline hsa_circ_0004970 & 1.17164435 & Up & 0.00191073 & $0.52606 \mid 24$ \\
\hline hsa_circ_0003760 & I. 19544727 & Up & 0.00830217 & 0.63723776 \\
\hline hsa_circ_0077040 & I.19660605 & Up & 0.00038828 & 0.36707996 \\
\hline hsa_circ_0064649 & 1.20323629 & Up & 0.00634396 & 0.63530743 \\
\hline hsa_circ_005792I & 1.22298163 & Up & 0.00658344 & $0.637 \mid 225$ \\
\hline hsa_circ_0010167 & 1.22782484 & Up & $0.0170556 \mid$ & 0.65626057 \\
\hline hsa_circ_0002626 & $1.237964 \mid 8$ & Up & 0.04850693 & 0.69864211 \\
\hline hsa_circ_00048I4 & $1.24697 \mid 28$ & Up & 0.01902014 & 0.66175152 \\
\hline hsa_circ_0073356 & 1.24865998 & Up & 0.0053345 & 0.61994831 \\
\hline hsa_circ_0045I 23 & 1.24884849 & Up & 0.02550036 & 0.6879809 I \\
\hline hsa_circ_0069213 & 1.25118583 & Up & 0.00366065 & 0.58881715 \\
\hline hsa_circ_0079548 & 1.25190508 & Up & 0.01484117 & 0.6461669 \\
\hline
\end{tabular}

(Continued) 
Table 2 (Continued).

\begin{tabular}{|c|c|c|c|c|}
\hline circRNAs & Log2FoldChange & Regulation & P-value & P-adj \\
\hline hsa_circ_0090098 & $|.2553684|$ & Up & 0.02596742 & 0.6886992 \\
\hline hsa_circ_0030624 & 1.25599738 & $U_{p}$ & 0.03651889 & 0.6896574 \\
\hline hsa_circ_0069324 & 1.26605445 & Up & 0.00060556 & 0.42191899 \\
\hline hsa_circ_0060335 & 1.28618107 & $U_{p}$ & $0.0008157 \mid$ & 0.44105722 \\
\hline hsa_circ_0058206 & $1.33626|6|$ & $U_{p}$ & 0.01802886 & 0.65626057 \\
\hline hsa_circ_0056882 & I.34999287 & $U_{p}$ & 0.03545574 & 0.6886992 \\
\hline hsa_circ_00|3437 & 1.36836499 & Up & 0.01654656 & $0.6547|98|$ \\
\hline hsa_circ_0046700 & 1.36944609 & $U_{p}$ & 0.0439923 & 0.69594076 \\
\hline hsa_circ_0082549 & 1.37107512 & $U_{p}$ & $9.10 \times 10^{-5}$ & 0.36011603 \\
\hline hsa_circ_0057436 & I.37990777 & $U_{p}$ & 0.00097797 & 0.47332912 \\
\hline hsa_circ_004887I & 1.42092532 & Up & 0.04047976 & $0.69077|5|$ \\
\hline hsa_circ_0009443 & 1.48425272 & Up & 0.00287515 & 0.52887377 \\
\hline hsa_circ_0060450 & $1.51788 \mid 73$ & Up & 0.01218226 & 0.6461669 \\
\hline hsa_circ_0060456 & 1.55691545 & Up & 0.04257115 & 0.69594076 \\
\hline hsa_circ_0026352 & 1.61091952 & $U_{p}$ & 0.0312629 & 0.6886992 \\
\hline hsa_circ_005662I & 1.65069395 & $U_{p}$ & 0.01137625 & 0.6461669 \\
\hline hsa_circ_0057880 & 1.65520964 & $U_{p}$ & 0.01050849 & 0.64298015 \\
\hline hsa_circ_0073340 & 1.72721874 & $U_{p}$ & 0.00133111 & 0.49533889 \\
\hline hsa_circ_0029674 & I.74809879 & $U_{p}$ & 0.00229149 & 0.52887377 \\
\hline hsa_circ_0021068 & $1.7969 \mid 824$ & Up & 0.00294272 & 0.52887377 \\
\hline hsa_circ_0053947 & 1.80953972 & $U_{p}$ & 0.02793328 & 0.6886992 \\
\hline hsa_circ_0002202 & 1.89666917 & $U_{p}$ & 0.00079917 & 0.44105722 \\
\hline hsa_circ_0079556 & 1.94764347 & $U_{p}$ & 0.00845628 & 0.63723776 \\
\hline hsa_circ_00I5729 & 2.96958438 & Up & 0.02245883 & 0.67318317 \\
\hline hsa_circ_0092238 & 3.03306764 & $U_{p}$ & 0.02362915 & 0.67886653 \\
\hline hsa_circ_0092236 & 3.45575278 & $U_{p}$ & 0.03065391 & 0.6886992 \\
\hline hsa_circ_009222I & 4.29199121 & $U_{p}$ & 0.03950529 & 0.68996252 \\
\hline
\end{tabular}

illustrated that hsa_circ_0002202 is mainly located in the cytoplasm in THP-1-derived macrophages, with the remaining proportion in the nucleus (Figure 7A). Transfection of si-circ significantly decreased hsa_circ_0002202 expression in THP-1-derived macrophages (Figure 7B). Afterward, qRT-PCR results showed that hsa_circ_0002202 knockdown significantly suppressed the expression of IFIT1, IFIH1, CXCL10, and iNOS under IFN-I stimulation, suggesting an inhibitory effect of hsa_circ_0002202 knockdown on IFN-I-induced inflammation (Figure 7C).

\section{Discussion}

Despite great efforts to find treatment strategies, T1DM is still a terrible threat to human health, and its prevalence is still rising worldwide. To better understand the exact mechanisms of T1DM and promote the discovery of new biomarkers, we analyzed the gene expression data from GEO public database. Furthermore, we established a circRNAlncRNA-miRNA-mRNA ceRNA regulatory network in T1DM, which elucidated the underlying pathogenesis of T1DM.

The post-transcriptional regulation of gene expression mediated by the ceRNA regulatory network during the diseases process has attracted wide attention from researchers. In diabetes and diabetic complications, the ceRNA regulatory network in pathophysiological processes has been widely reported. For example, lncRNA MT1P3 promoted p2y12 expression by sponging miR-126 to promote platelet activation and aggregation in type 2 diabetes. $^{45}$ lncRNA H2k2/miR-449a/b/Trim11 signaling pathway promotes mesangial cell proliferation in diabetic nephropathy. ${ }^{46}$ Hsa_circ_010567/miR-141/TGF- $\beta 1$ axis promotes myocardial fibrosis in diabetic mice. ${ }^{47}$ The complex interaction network of multiple factors may be more theoretically meaningful for revealing the internal mechanism of T1DM. But few studies focused on the ceRNA 
Table 3 Results of Gene Ontology (GO) Enrichment Analysis of Differentially Expressed circRNAs

\begin{tabular}{|c|c|c|c|c|}
\hline Ontology & Term & Description & Gene Number & P-adj \\
\hline BP & GO:0030199 & Collagen fibril organization & 7 & 0.00044787 \\
\hline BP & GO:0043062 & extracellular structure Organization & 15 & 0.00076629 \\
\hline BP & GO:0030198 & Extracellular matrix organization & 14 & 0.00076629 \\
\hline BP & GO:0060840 & Artery development & 7 & 0.00694781 \\
\hline BP & GO:007I230 & Cellular response to amino acid stimulus & 6 & 0.00694781 \\
\hline BP & GO:0043200 & Response to amino acid & 7 & 0.01121075 \\
\hline BP & GO:000II0I & Response to acid chemical & 11 & 0.02282114 \\
\hline BP & GO:0045I76 & Apical protein localization & 3 & 0.02282114 \\
\hline $\mathrm{CC}$ & GO:0045I2I & Membrane raft & 11 & 0.00284762 \\
\hline $\mathrm{CC}$ & GO:0098857 & Membrane microdomain & 11 & 0.00284762 \\
\hline $\mathrm{CC}$ & GO:0098589 & Membrane region & 11 & 0.00284762 \\
\hline $\mathrm{CC}$ & GO:0044420 & Extracellular matrix component & 5 & 0.00284762 \\
\hline $\mathrm{CC}$ & GO:0005583 & Fibrillar collagen trimer & 3 & 0.00333723 \\
\hline CC & GO:0098643 & Banded collagen fibril & 3 & 0.00333723 \\
\hline CC & GO:0005788 & Endoplasmic reticulum lumen & 10 & 0.00425887 \\
\hline CC & GO:0031252 & Cell leading edge & $\mathrm{II}$ & 0.0074448 I \\
\hline $\mathrm{CC}$ & GO:000590I & Caveola & 5 & 0.01094539 \\
\hline $\mathrm{CC}$ & GO:0098644 & Complex of collagen trimers & 3 & 0.01126914 \\
\hline CC & GO:000558I & Collagen trimer & 5 & 0.01321773 \\
\hline $\mathrm{CC}$ & GO:0045I77 & Apical part of cell & 10 & 0.01385878 \\
\hline CC & GO:0016324 & Apical plasma membrane & 9 & 0.01385878 \\
\hline CC & GO:0062023 & Collagen-containing extracellular matrix & 10 & 0.01868493 \\
\hline $\mathrm{CC}$ & GO:0031253 & Cell projection membrane & 9 & 0.02141473 \\
\hline CC & GO:0044853 & Plasma membrane raft & 5 & 0.02530242 \\
\hline $\mathrm{CC}$ & GO:0031256 & Leading edge membrane & 6 & 0.03027327 \\
\hline CC & GO:0005844 & Polysome & 4 & 0.03442097 \\
\hline $\mathrm{CC}$ & GO:00059II & Cell-cell junction & 10 & 0.03442097 \\
\hline $\mathrm{CC}$ & GO:0097470 & Ribbon synapse & 2 & 0.03835081 \\
\hline $\mathrm{CC}$ & GO:0005925 & Focal adhesion & 9 & 0.04570731 \\
\hline CC & GO:0005924 & Cell-substrate adherens junction & 9 & 0.04581649 \\
\hline $\mathrm{CC}$ & GO:0030055 & Cell-substrate junction & 9 & 0.04674156 \\
\hline MF & GO:0030020 & Extracellular matrix structural constituent conferring tensile strength & 5 & 0.00708066 \\
\hline MF & GO:000520I & Extracellular matrix structural constituent & 8 & 0.00916654 \\
\hline MF & GO:0I40I04 & Molecular carrier activity & 5 & 0.00916654 \\
\hline MF & GO:0019838 & Growth factor binding & 7 & 0.01134742 \\
\hline MF & GO:0046332 & SMAD binding & 5 & 0.03542754 \\
\hline MF & GO:0003779 & Actin binding & 11 & 0.04505555 \\
\hline
\end{tabular}

Abbreviations: BP, biological process; CC, cellular component; MF, molecular function.

Table 4 Results of KEGG Enrichment Analysis of Differentially Expressed circRNAs

\begin{tabular}{|l|l|l|l|}
\hline Term & Description & Gene Number & P-adj \\
\hline hsa04974 & Protein digestion and absorption & 7 & 0.009083535 \\
\hline
\end{tabular}

regulatory networks in T1DM. Meanwhile, previous studies on T1DM ceRNA regulatory networks only discussed the role of either $\operatorname{lncRNA}{ }^{48}$ or circRNA, ${ }^{33}$ and did not consider both of them simultaneously. Therefore, this study was systematic and comprehensive for revealing the T1DM mechanism.

In the current study, we identified 178 DEcircRNAs, 404 DElncRNAs, and 73 DEmRNAs from the 


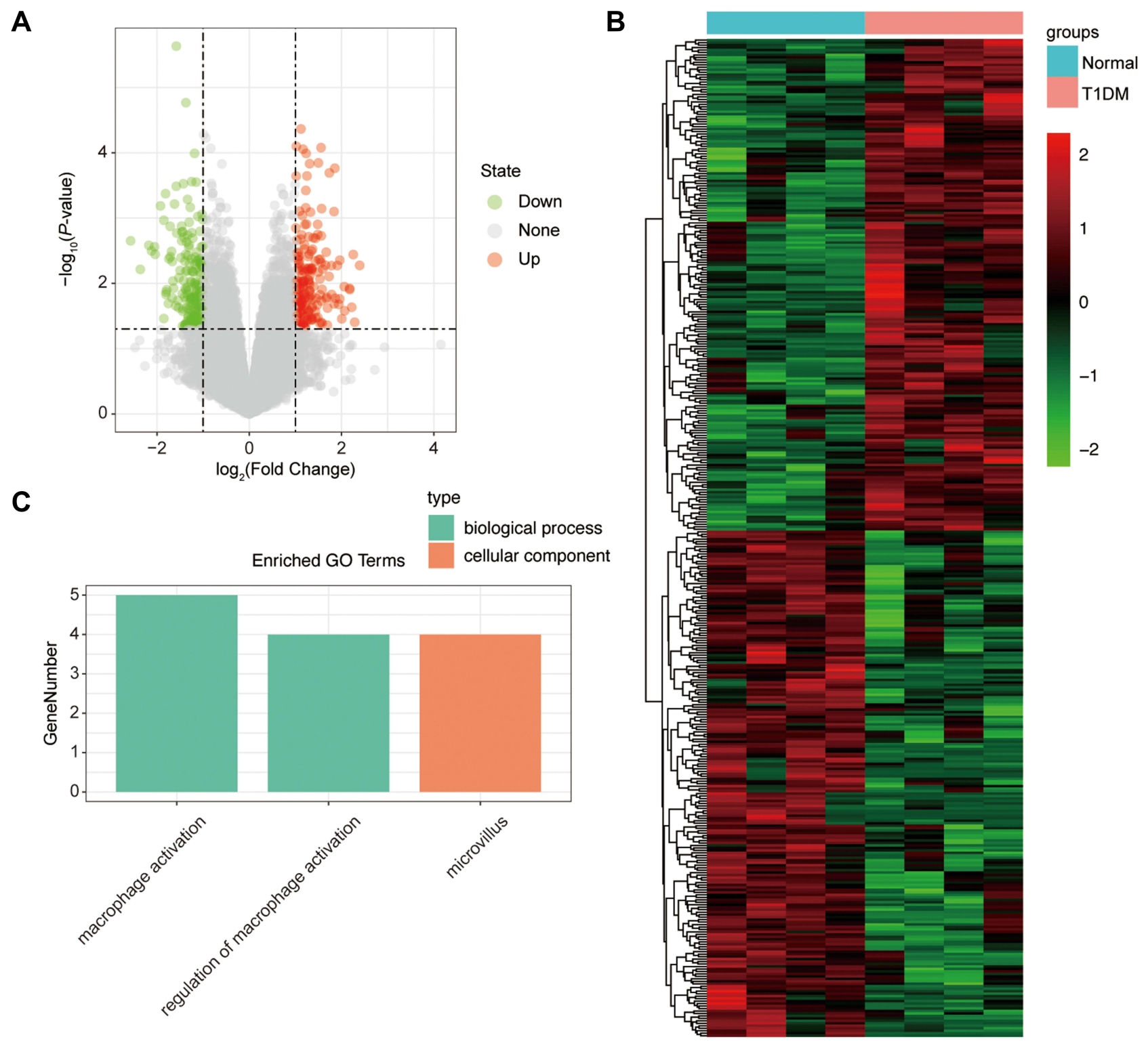

Figure 3 The differentially expressed IncRNAs in TIDM and healthy patients. (A) Volcano plot of DElncRNAs between normal and TIDM groups. Red and green indicate up- and downregulation, respectively. (B) Heatmap of DElncRNAs between normal and TIDM groups. Red color represents increased expression, and green color represents decreased expression. The darker the color, the greater the difference of IncRNA expression. (C) Enrichment analysis of the DElncRNAs in the categories biological process and cellular component.

GSE133225 database. Meanwhile, we analyzed the miRNA chip GSE133217 to construct the interaction network of circRNA, IncRNA, miRNA, and mRNA. This network suggested that circRNA and lncRNA play a central regulatory role in T1DM. One circRNA and IncRNA can be associated with multiple identical miRNAs to regulate more mRNAs synergistically. Among the circRNAs and lncRNAs, circRNA hsa_circ_0002202 and lncRNA AL356740.2-201 (ENST00000601559.1) linked most miRNAs. Here, we selected hsa_circ_0002202 for the in vitro experiment verification. Hsa_circ_0002202 is formed from SMAD4A. A previous study has reported the oncogene role of circSAMD4A in osteosarcoma. ${ }^{49}$ Liu et al found that circSAMD4A acts as an adipogenesis promoting factor. ${ }^{50}$ In this study, we found that hsa_circ_0002202 was up-regulated in T1DM PBMCs samples, suggesting that hsa_circ_0002202 may take part in the T1DM procession. As the circRNAs show their sponge function in the cytoplasm, ${ }^{26}$ we detected 
Table 5 Differentially Expressed IncRNAs Between the Control Group and TIDM Group

\begin{tabular}{|c|c|c|c|c|}
\hline IncRNAs & Log2FoldChange & Regulation & P-value & P-adj \\
\hline Inc-MARCI-2:I & -2.571855 & Down & 0.002228 & 0.49624236 \\
\hline Inc-PSMG I-3:2 & -2.3628597 & Down & 0.00610319 & 0.55695089 \\
\hline CCND2-ASI-20I & -2.1767994 & Down & 0.00261656 & 0.51224337 \\
\hline Inc-ANGPTL2-7:I & -2.1177664 & Down & 0.00318539 & 0.53930742 \\
\hline AL356740.2-20I & -2.0532982 & Down & 0.0027546 & 0.51224337 \\
\hline Inc-APBA2-1:3 & -2.0384649 & Down & 0.00358789 & 0.54892347 \\
\hline Inc-SELIL3-I0:I & -1.9247783 & Down & $0.00065 \mid 27$ & 0.39679443 \\
\hline ENPP7PI3 & -1.8523605 & Down & 0.03454029 & 0.72695785 \\
\hline Inc-MBNL2-2:I & -1.8516299 & Down & 0.00108059 & 0.42609515 \\
\hline Inc-CTD-2523DI3.I.I-2:3 & -1.8151432 & Down & 0.00042329 & 0.33998788 \\
\hline LOCI0I929733 & -1.805357 & Down & 0.01482033 & 0.65476375 \\
\hline ACOII 287.I-203 & -1.8043227 & Down & 0.01357893 & 0.63919136 \\
\hline ACOI3553.4-20I & -1.7883164 & Down & 0.01251521 & 0.63775317 \\
\hline AL02I368.3-20I & -1.7524563 & Down & 0.02035199 & 0.68302181 \\
\hline Inc-CD5L-I:I & -1.7425319 & Down & 0.00420523 & 0.55695089 \\
\hline SEMA6A-ASI-206 & -1.7140352 & Down & 0.00134151 & 0.4460612 \\
\hline LINC00299-203 & -1.71106 & Down & 0.00391051 & 0.55546359 \\
\hline Inc-FRZB-2:2 & -1.6847279 & Down & 0.00324333 & 0.53930742 \\
\hline GGNBPI & $-1.67 \mid 9447$ & Down & 0.0166596 & 0.65620897 \\
\hline Inc-UNC5CL-7:I & -1.6687674 & Down & 0.00461045 & 0.55695089 \\
\hline Inc-MRPS5-I3:I & -1.6546035 & Down & 0.00921325 & 0.6151086 \\
\hline Inc-Clorf64-I:I & -1.6395822 & Down & 0.01190126 & 0.63705043 \\
\hline ALI36979.I-20I & $-|.620287|$ & Down & 0.0006092 & 0.39679443 \\
\hline Inc-CNGBI-I:3 & -1.5925938 & Down & 0.02212688 & 0.6889443 \\
\hline AL02I937.6-20I & -1.5827605 & Down & 0.00032605 & 0.33391458 \\
\hline Inc-RASGRPI-6:I & -1.5821617 & Down & $2.33 \times 10^{-6}$ & 0.05960188 \\
\hline Inc-ANO2-4:I & -1.5733225 & Down & 0.00170014 & 0.48365219 \\
\hline LINC0047I & -1.5517992 & Down & 0.01828625 & 0.66352772 \\
\hline AP00I363.I-20I & -1.5323863 & Down & 0.02355621 & 0.69616967 \\
\hline ACOII405.I-20I & -1.523797 & Down & 0.00820535 & I \\
\hline ACI08935.I-20I & -1.5001012 & Down & 0.00635053 & 0.55695089 \\
\hline Inc-TBCID3C-4:I & -1.4786853 & Down & 0.00403532 & 0.55546359 \\
\hline Inc-LIPI-6:I & -1.4669252 & Down & 0.00132802 & 0.4460612 \\
\hline Inc-FCHSD2-4:I & -1.4647594 & Down & 0.00223289 & 0.49624236 \\
\hline ACI04590.I-20I & -1.458406 & Down & 0.00163683 & 0.47784335 \\
\hline Inc-NDFIP2-8:I & -1.4500676 & Down & 0.01442379 & 0.65361455 \\
\hline Inc-AL359878.I-I:I & -1.4473997 & Down & 0.04508299 & 0.74859413 \\
\hline Inc-NUP98-2:I & -1.4448238 & Down & 0.02667345 & 0.70331657 \\
\hline Inc-DTHDI-2:I & $-1.444 \mid 469$ & Down & 0.00161419 & 0.47784335 \\
\hline Inc-DCAF4LI-I:I & -1.4367953 & Down & 0.02450173 & 0.69781796 \\
\hline AC022296.I-20I & $-1.4277 \mid 72$ & Down & 0.00029813 & 0.33391458 \\
\hline LINC02279-20I & -1.4213164 & Down & $0.0090728 I$ & 0.6151086 \\
\hline C5orf66-ASI & -1.4124554 & Down & 0.00199206 & 0.49624236 \\
\hline Inc-IRF5-2:I & $-1.40879 \mid 8$ & Down & 0.04752968 & 0.75874445 \\
\hline Inc-FXYD2-I:2 & $-1.40|659|$ & Down & 0.01605233 & 0.65476375 \\
\hline ENST00000434742 & -1.3965605 & Down & 0.04266873 & 0.74199989 \\
\hline LINC007I0-2I0 & -1.3926583 & Down & 0.00459619 & 0.55695089 \\
\hline Inc-RUNXITI-6:I & -1.390228 & Down & 0.04310897 & 0.74348205 \\
\hline Inc-REPI5-2:I & $-1.3887 \mid 83$ & Down & 0.01204392 & 0.63775317 \\
\hline
\end{tabular}

(Continued) 
Table 5 (Continued).

\begin{tabular}{|c|c|c|c|c|}
\hline IncRNAs & Log2FoldChange & Regulation & P-value & P-adj \\
\hline Inc-GLCCII-5:I & -1.3790449 & Down & $0.0036005 \mathrm{I}$ & 0.54892347 \\
\hline Inc-TDRD5-2:2 & $-1.3779|4|$ & Down & 0.00653613 & $0.5634496 \mathrm{I}$ \\
\hline Inc-TIPARP-I:I & -1.3745656 & Down & $1.71 \times 10^{-5}$ & 0.219024 \\
\hline NONHSATI 44810 & -1.3733309 & Down & 0.04223393 & 0.74199989 \\
\hline Inc-C8orf| 2-I:5 & -1.3670614 & Down & 0.01262556 & 0.63775317 \\
\hline FLJ42393-20I & -1.360109 & Down & 0.01787249 & 0.65667837 \\
\hline AL03II I86.I-20I & -1.359927 & Down & 0.00231239 & 0.50172959 \\
\hline NONHSAT0782I 7 & -1.3517092 & Down & 0.03830547 & 0.73920545 \\
\hline HSFYIPI & -1.3512769 & Down & 0.02395161 & 0.69616967 \\
\hline AL365295.I-203 & -1.3483624 & Down & 0.00642502 & 0.55846139 \\
\hline Inc-ST8SIA4-9:I & -1.3434237 & Down & $0.0427|07|$ & 0.74199989 \\
\hline LINC0I46I & -1.3413799 & Down & 0.01143714 & 0.63705043 \\
\hline Inc-GPR27-I6:I & -1.3407603 & Down & 0.01468499 & 0.65476375 \\
\hline AC005772.I-20I & -1.3381628 & Down & 0.00051768 & 0.37869313 \\
\hline MIR2II7 & -1.3345777 & Down & 0.00182095 & 0.49597572 \\
\hline Inc-GPR I80-8:I & -1.3325294 & Down & 0.04785484 & 0.75909133 \\
\hline Inc-CDHI9-I:I & -1.32977|| & Down & 0.00373595 & 0.55546359 \\
\hline Inc-MYLIP-I:I & -1.3230214 & Down & 0.03290443 & 0.7208645 \\
\hline Inc-AC006|56.I-6:I & -1.3212148 & Down & 0.03163903 & 0.72031743 \\
\hline LINC00326-202 & $-1.3|3| 65 \mid$ & Down & 0.002781 & 0.51224337 \\
\hline Inc-THSD7A-5:I & -1.3104044 & Down & 0.01270662 & 0.63775317 \\
\hline AL5I3320.I-20I & -1.3078099 & Down & 0.02483242 & 0.69822202 \\
\hline LOCI00288570 & -1.302538 & Down & 0.00116144 & 0.42609515 \\
\hline OR4FI3P & -1.3002494 & Down & 0.00258605 & 0.51224337 \\
\hline SKAPI-ASI-20I & -1.2965689 & Down & 0.0399885 & 0.74199989 \\
\hline Inc-ZC3HI2B-8:I & -1.2960126 & Down & 0.00485753 & 0.55695089 \\
\hline Inc-DDX58-6:2 & -1.2919894 & Down & 0.04664872 & 0.75453224 \\
\hline Inc-MRPL9-I:I & -1.2821415 & Down & 0.0193777 & 0.67753348 \\
\hline Inc-C6orfl 95-20:I & -1.2797904 & Down & 0.00068424 & 0.39679443 \\
\hline LINC00563 & -1.2751075 & Down & 0.04014392 & 0.74199989 \\
\hline RNU6-693P-20I & -1.2734215 & Down & 0.00931818 & 0.6151086 \\
\hline Inc-SERP2-I3:I & -1.2711219 & Down & 0.03525604 & 0.72695785 \\
\hline Inc-NKD2-3:8 & -1.2692144 & Down & $0.0174 \mid 431$ & 0.65667837 \\
\hline Inc-CSNKIAI-6:I & -1.2661357 & Down & 0.03246677 & 0.72031743 \\
\hline Inc-TMEMI78-4:I & -1.2653338 & Down & 0.01654808 & 0.65588218 \\
\hline ACOI0280.I-20I & -1.2633516 & Down & 0.02288955 & 0.69353994 \\
\hline ACOII333.I-20I & -1.2601339 & Down & 0.01734914 & 0.65667837 \\
\hline Inc-SRY-I2:I & -1.255766 & Down & 0.04179039 & 0.74199989 \\
\hline LOCI0192954I & -1.2543068 & Down & 0.00422143 & 0.55695089 \\
\hline Inc-IQCG-I:I & -1.2510293 & Down & 0.00960776 & 0.6151086 \\
\hline Inc-TRAF7-I:4 & -1.250778 & Down & 0.02651159 & 0.7025969 \\
\hline AC06805I.I-202 & -1.250693 & Down & 0.01630728 & 0.65588218 \\
\hline Inc-WSBI-9:I & -1.2499003 & Down & 0.00027697 & 0.33391458 \\
\hline HIPKI-ASI-202 & -1.247658 & Down & 0.00646044 & $0.5588059 \mid$ \\
\hline Inc-ANKRD65-4:I & -1.2456674 & Down & 0.00167491 & $0.48 \mid 82856$ \\
\hline Inc-RASDI-2:I & -1.2336926 & Down & 0.03531618 & 0.72695785 \\
\hline Inc-PTPNI2-I:I & -1.2251669 & Down & 0.00203611 & 0.49624236 \\
\hline Inc-ASAH2B-6:I & $-|.224933|$ & Down & 0.02282058 & 0.69285761 \\
\hline Inc-VSTM2B-7:I & -1.2187025 & Down & 0.00664315 & 0.56471029 \\
\hline
\end{tabular}

(Continued) 
Table 5 (Continued).

\begin{tabular}{|c|c|c|c|c|}
\hline IncRNAs & Log2FoldChange & Regulation & P-value & P-adj \\
\hline AC009I45.3-20I & -1.2150544 & Down & 0.00669705 & 0.56471029 \\
\hline Inc-EXOC4-2:I & -1.2094249 & Down & 0.03638874 & 0.73000405 \\
\hline Inc-ASFIA-7:I & $-1.209|28|$ & Down & 0.04945525 & 0.76055974 \\
\hline LOCI01927915 & -1.2090211 & Down & 0.04151546 & 0.74199989 \\
\hline MIR520D & -1.2003733 & Down & 0.01614125 & 0.65476375 \\
\hline Inc-RPII-I48O2I.3.I-3:I & -1.1989594 & Down & 0.03101553 & 0.72031743 \\
\hline Inc-VCPIPI-2:I & -1.1940207 & Down & 0.01680361 & 0.65667837 \\
\hline Inc-MANEA-I5:I & -1.1934022 & Down & 0.03073549 & 0.71864918 \\
\hline AP0072I6.I-20I & -1.1933507 & Down & 0.03903039 & 0.74199989 \\
\hline Inc-BOC-I:I & -1.1894086 & Down & 0.03214827 & 0.72031743 \\
\hline Inc-ALCAM-I6:I & -1.1864006 & Down & 0.00010229 & 0.23823842 \\
\hline AC243965.I-20I & -1.1825172 & Down & 0.00992456 & 0.61994889 \\
\hline Inc-SNRPEP2-I:I & -1.1777925 & Down & 0.00505178 & 0.55695089 \\
\hline MIR8063 & -1.1720035 & Down & 0.03554046 & 0.72695785 \\
\hline LINC0I497 & -1.1712102 & Down & 0.00396132 & 0.55546359 \\
\hline AP005202.I-20I & -1.1711452 & Down & 0.02687222 & 0.70508418 \\
\hline Inc-CA5A-I4:I & -1.170243 & Down & 0.01880508 & 0.66963349 \\
\hline TBCID26-ASI-20I & -1.1690859 & Down & 0.03794326 & 0.73763201 \\
\hline Inc-RHOB-6:4 & -1.164896 & Down & 0.00952576 & 0.6151086 \\
\hline Inc-TMEM235-I:I & $-1.164446 \mid$ & Down & 0.02080375 & 0.68520443 \\
\hline Inc-NKAIN3-I:I & -1.1639521 & Down & 0.01613077 & 0.65476375 \\
\hline Inc-TFAP2A-I2:I & -1.1638797 & Down & $0.045838 \mathrm{I}$ & $0.752507 \mathrm{II}$ \\
\hline NONHSAT080748 & -1.1637059 & Down & 0.04242998 & 0.74199989 \\
\hline Inc-WDRI-2:I & $-1.16|243|$ & Down & 0.01413228 & $0.646 \mid 2284$ \\
\hline Inc-STYKI-2:I & -1.160814 & Down & 0.03332132 & 0.72302337 \\
\hline Inc-C2CD4A-8:3 & $-|.1589| 7 \mid$ & Down & 0.03249489 & 0.72031743 \\
\hline Inc-RAB9A-4:I & -1.1570636 & Down & 0.04423563 & 0.7477493 I \\
\hline Inc-GRIK4-2:I & -1.1570003 & Down & 0.01043825 & 0.62720663 \\
\hline AL356/24.I-2I2 & -1.1529537 & Down & 0.0059984 & 0.55695089 \\
\hline Inc-GRIDI-I0:2 & -1.1509303 & Down & 0.00028173 & 0.33391458 \\
\hline Inc-DMRTAI-2I:I & $-1 .|49458|$ & Down & 0.02080202 & 0.68520443 \\
\hline COPG2ITI & $-I .|48850|$ & Down & 0.03280207 & 0.7208645 \\
\hline GUSBPI & -1.1442627 & Down & 0.03300713 & 0.7208645 \\
\hline Inc-AC0I625I.I-I8:I & $-1.1426 \mid 14$ & Down & 0.01124953 & 0.63705043 \\
\hline Inc-IGHMBP2-2:I & -1.1410125 & Down & 0.01522257 & 0.65476375 \\
\hline Inc-CLKI-5:I & -1.1408544 & Down & 0.00456639 & 0.55695089 \\
\hline Inc-DDIT4L-I:I & -1.139392 & Down & 0.0033263 & 0.54489032 \\
\hline Inc-ATP8A2-4:I & $-1.130985 \mid$ & Down & 0.01285546 & 0.63775317 \\
\hline AC002550.I-20I & -1.1258339 & Down & 0.03121112 & 0.72031743 \\
\hline Inc-BX255923.I-4:I & $-|.125447|$ & Down & 0.02958836 & 0.71263333 \\
\hline LRRC37A6P & -1.1246433 & Down & 0.01039373 & 0.62720663 \\
\hline Inc-THBD-3:I & -1.1181096 & Down & 0.01823629 & 0.66321547 \\
\hline SMIM2-ITI & -1.1152686 & Down & 0.04821159 & 0.75909133 \\
\hline Inc-EIF2AK3-I0:I & -1.1129693 & Down & 0.00094609 & 0.41823037 \\
\hline AC072039.2-20I & -1.1023779 & Down & 0.04427089 & 0.7477493 I \\
\hline AC073655.I-20I & -1.1010143 & Down & 0.00438297 & 0.55695089 \\
\hline Inc-HECA-8:3 & $-I .100756 \mid$ & Down & 0.01730192 & 0.65667837 \\
\hline Inc-CAMK2N2-I:I & -1.100534 & Down & 0.02254575 & $0.69|304| 2$ \\
\hline
\end{tabular}

(Continued) 
Table 5 (Continued).

\begin{tabular}{|c|c|c|c|c|}
\hline IncRNAs & Log2FoldChange & Regulation & P-value & P-adj \\
\hline Inc-RET-2:I & -1.0994363 & Down & 0.02204275 & 0.6889443 \\
\hline AC006482.I-20I & -1.0943276 & Down & 0.01606638 & 0.65476375 \\
\hline NONHSAT066757 & -1.0893525 & Down & 0.02619728 & 0.7025969 \\
\hline Inc-PCIFI-I:I & -1.0831588 & Down & 0.03331068 & 0.72302337 \\
\hline Inc-RGS9-1:9 & -1.0808278 & Down & 0.04328182 & 0.74348205 \\
\hline Inc-TMEM63B-3:I & -1.0806828 & Down & 0.03297302 & 0.7208645 \\
\hline AL354726.I-20I & -1.0779947 & Down & 0.03804582 & 0.7382395 \\
\hline Inc-VPS45-5:I & -1.0767237 & Down & 0.03551093 & 0.72695785 \\
\hline MIRI00HG-204 & -1.0749286 & Down & 0.00478367 & 0.55695089 \\
\hline Inc-ERMN-3:I & -1.0724303 & Down & 0.01323341 & 0.63919136 \\
\hline Inc-POMI2ILI2-9:I & $-1.070 \mid 442$ & Down & 0.01424926 & $0.6479997 \mid$ \\
\hline LINC02399-20I & -1.0677909 & Down & 0.00159699 & 0.47784335 \\
\hline ZNF385D-AS2 & $-1.06750 \mid 4$ & Down & 0.03541215 & 0.72695785 \\
\hline Inc-CCNJL-3:I & -1.0661945 & Down & 0.03740759 & 0.73503181 \\
\hline Inc-SLC4A3-7:4 & -1.0657752 & Down & 0.00420941 & 0.55695089 \\
\hline AC096666.I-20I & -1.0645687 & Down & 0.0475234 & 0.75874445 \\
\hline TRAPPCI2-ASI-20I & -1.0621034 & Down & 0.00527019 & 0.55695089 \\
\hline AC0I0973.I-20I & -1.0620397 & Down & 0.01113385 & 0.63705043 \\
\hline Inc-PYGOI-3:I & -1.0617362 & Down & $0.0073504 I$ & 0.57770209 \\
\hline NONHSATI06I26 & -1.0615194 & Down & 0.00454088 & 0.55695089 \\
\hline Inc-ZNF33A-7:I & -1.0611652 & Down & 0.02394318 & 0.69616967 \\
\hline AC02I097.I-20I & $-1.060772 \mid$ & Down & 0.00678068 & 0.56733936 \\
\hline Inc-SNXII-9:I & -1.0565877 & Down & 0.04932529 & 0.75985275 \\
\hline LINC00092-202 & -1.0542115 & Down & 0.00493655 & 0.55695089 \\
\hline LOCI00I304I7 & $-1.044 \mid 384$ & Down & 0.02061929 & 0.68461156 \\
\hline Inc-DACTI-2:I & -1.0422563 & Down & 0.00276522 & 0.51224337 \\
\hline Inc-CACNAIE-5:3 & -1.0418233 & Down & 0.032085 & 0.72031743 \\
\hline Inc-KCNJ2-2:I & -1.0409158 & Down & 0.03770802 & 0.73763201 \\
\hline ZNF45I-ASI-202 & $-1.0407 \mid 45$ & Down & 0.0199397 & 0.68003654 \\
\hline AC022509.I-203 & -1.0393619 & Down & 0.04632896 & 0.75407344 \\
\hline ALI62253.I-20I & -1.039287 & Down & 0.04553105 & 0.75046636 \\
\hline LINC0I339 & $-1.037676 \mid$ & Down & 0.01322858 & 0.63919136 \\
\hline RNU4-30P-20I & -1.03405 & Down & 0.04484526 & 0.74859413 \\
\hline Inc-ZNF527-3:I & -1.0331657 & Down & 0.00495913 & 0.55695089 \\
\hline Inc-VSTM4-I:3 & -1.0311378 & Down & 0.03884105 & 0.74199989 \\
\hline Inc-ORMDL2-3:I & -1.0308522 & Down & 0.03578571 & $0.728316 \mathrm{I}$ \\
\hline Inc-AKIPI-5:I & -1.0271559 & Down & 0.02023333 & 0.68302181 \\
\hline AL35909I.4-20I & -1.0270896 & Down & 0.04632883 & 0.75407344 \\
\hline Inc-PPIAL4G-24:I & -1.0236308 & Down & 0.02813286 & 0.70952942 \\
\hline LOCI00506679 & -1.0230377 & Down & 0.00087856 & 0.41823037 \\
\hline Inc-TSSCI-I2:I & -1.0215854 & Down & 0.00560415 & 0.55695089 \\
\hline LOCI00505984 & -1.0208834 & Down & 0.0255792 & 0.7025969 \\
\hline Inc-FAM50B-I2:I & -1.0196309 & Down & 0.02412553 & 0.69616967 \\
\hline Inc-ALG9-I:I & $-1.017695 \mid$ & Down & 0.00552229 & 0.55695089 \\
\hline AC096669.I-20I & -1.0157852 & Down & 0.03891937 & 0.74199989 \\
\hline Inc-ZNFI35-I:I & -1.0126176 & Down & 0.02399892 & 0.69616967 \\
\hline Inc-C2orf65-7:I & -1.0125274 & Down & 0.03958279 & 0.74199989 \\
\hline NONHSAT093043 & -1.0122218 & Down & 0.0244619 & 0.69781796 \\
\hline Inc-MARCKS-2:I & -1.0108829 & Down & 0.01484675 & 0.65476375 \\
\hline
\end{tabular}

(Continued) 
Table 5 (Continued).

\begin{tabular}{|c|c|c|c|c|}
\hline IncRNAs & Log2FoldChange & Regulation & P-value & P-adj \\
\hline Inc-ERII-5:I & -1.0096415 & Down & 0.00105398 & 0.42609515 \\
\hline Inc-AC0I| $484 . I-I: I$ & -1.0085847 & Down & 0.02523925 & 0.69822202 \\
\hline Inc-CI5orf4I-I6:I & -1.0085698 & Down & 0.00270442 & 0.51224337 \\
\hline AC006II6.7-20I & -1.0082255 & Down & 0.0092013 & 0.6151086 \\
\hline AL354760.I-20I & -1.007775 & Down & 0.02302132 & $0.6936895 \mathrm{I}$ \\
\hline Inc-HS3ST3AI-6:I & -1.005627 & Down & 0.01277075 & 0.63775317 \\
\hline LINC0I007 & -1.0051638 & Down & $0.04 I 74436$ & 0.74199989 \\
\hline Inc-FSTL5-2:I & -1.0044016 & Down & 0.02732332 & 0.70738927 \\
\hline Inc-ORI0G2-7:I & -1.0040498 & Down & 0.02589504 & 0.7025969 \\
\hline Inc-SCNIIA-I:3 & -1.0003752 & Down & 0.01675468 & 0.65667837 \\
\hline KRT8P4I & 1.00323605 & Up & 0.0157024 & 0.65476375 \\
\hline Inc-TOMM70A-2:I & $1.00437 \mid I I$ & $U_{p}$ & $0.0209401 \mathrm{I}$ & 0.68579407 \\
\hline ST20-ASI-20I & 1.00542536 & $U_{p}$ & 0.00950719 & 0.6151086 \\
\hline Inc-MCM3AP-2:I & 1.00595783 & Up & 0.04176408 & 0.74199989 \\
\hline NONHSATI 03382 & 1.00610895 & Up & 0.01949203 & 0.67753348 \\
\hline Inc-ZNF729-I:I & $1.008780 \mid 4$ & Up & 0.00141575 & $0.46 \mid 26795$ \\
\hline Inc-ZNF6|3-I:5 & $1.0088 \mid 485$ & Up & 0.00992771 & 0.61994889 \\
\hline ZKSCAN7-ASI-20I & 1.01240919 & Up & 0.01157844 & 0.63705043 \\
\hline LINC00I73 & $1.0140048 \mid$ & Up & 0.04488089 & $0.748594 I 3$ \\
\hline Inc-SCARFI-I:I & 1.0156664 & Up & 0.00981816 & 0.61762722 \\
\hline NONHSAT0I8354 & 1.01593666 & Up & 0.03530828 & 0.72695785 \\
\hline Inc-FMN2-5:I & 1.01602171 & Up & 0.0002276 & 0.32373463 \\
\hline Inc-SGIPI-3:I & 1.01645757 & $U_{p}$ & 0.01641438 & 0.65588218 \\
\hline LINC0I640-20I & 1.01688724 & $U_{p}$ & 0.04089227 & 0.74199989 \\
\hline AC09I906.I-20I & 1.01953621 & Up & $0.0327 \mid 348$ & 0.7208645 \\
\hline Inc-FAM98A-4:I & $1.0202178 \mid$ & $U_{p}$ & $7.97 \times 10^{-5}$ & 0.23823842 \\
\hline LINC0253I-20I & 1.0290261 & Up & 0.0217835 & 0.6875777 \\
\hline Inc-MKKS-2:I & 1.02955928 & Up & 0.0127837 & 0.63775317 \\
\hline Inc-VPS45-I:I & 1.03014965 & Up & 0.02882574 & 0.71150768 \\
\hline Inc-ATP6AP2-5:I & 1.03058486 & Up & 0.01059718 & 0.62720663 \\
\hline Inc-SSXI-3:I & 1.03120136 & $U_{p}$ & 0.00345991 & 0.54892347 \\
\hline Inc-TMEMI44-6:I & 1.03146297 & Up & 0.00218147 & 0.49624236 \\
\hline AC064805.I-203 & 1.03207632 & Up & 0.01476632 & 0.65476375 \\
\hline AL04978I.I-20I & 1.03367342 & Up & 0.03188715 & 0.72031743 \\
\hline Inc-CD38-5:I & $1.03537 \mid 23$ & Up & $0.024 \mid 3739$ & 0.69616967 \\
\hline Inc-RDHI0-3:I & 1.0354704 & Up & 0.00905218 & 0.6151086 \\
\hline MIR527 & 1.03634444 & Up & 0.04629443 & 0.75407344 \\
\hline Inc-IL36B-3:I & 1.04032276 & Up & 0.03473821 & 0.72695785 \\
\hline Inc-AP3SI-8: I & 1.04706344 & Up & 0.01681226 & 0.65667837 \\
\hline Inc-AC00438I.6.I-2:2 & 1.04706933 & Up & 0.03502406 & 0.72695785 \\
\hline Inc-ARLI I-I:I & $1.05|2366|$ & Up & 0.04263733 & 0.74199989 \\
\hline Inc-DEFB | |6-8:I & 1.05366198 & Up & 0.02045757 & $0.68302|8|$ \\
\hline Inc-COBLLI-2:I & $1.053738 \mid 8$ & Up & 0.00428499 & 0.55695089 \\
\hline Inc-TLXI-6:4 & $1.05385 \mid 44$ & Up & 0.02973516 & 0.71383843 \\
\hline Inc-FAMI35A-3:I & 1.0543549 & Up & 0.00588203 & 0.55695089 \\
\hline Inc-TFF3-2:I & 1.0556941 & Up & 0.00929413 & 0.6151086 \\
\hline Inc-NBPF6-I:5 & 1.06191301 & Up & 0.0039231 & 0.55546359 \\
\hline LINC00470-208 & I.06395774 & $U_{p}$ & 0.02171233 & 0.6875777 \\
\hline Inc-FREM3-4:I & 1.06877234 & $U_{p}$ & 0.03813847 & 0.7386227 \\
\hline
\end{tabular}

(Continued) 
Table 5 (Continued).

\begin{tabular}{|c|c|c|c|c|}
\hline IncRNAs & Log2FoldChange & Regulation & P-value & P-adj \\
\hline Inc-ZAP70-2:44 & 1.0688365 & Up & 0.01504184 & 0.65476375 \\
\hline Inc-ZSCAN5A-I:I & 1.06985443 & Up & 0.01950186 & 0.67753348 \\
\hline Inc-DDHDI-7:I & 1.07032663 & Up & 0.00334132 & 0.54489032 \\
\hline TMEMI8-DT-20I & 1.07452939 & Up & 0.00488947 & 0.55695089 \\
\hline ENST000006 | 4227 & 1.07841048 & Up & 0.00384573 & 0.55546359 \\
\hline Inc-NADKDI-2:I & 1.07972767 & Up & 0.02298006 & 0.69368951 \\
\hline Inc-WDR96-3:I & 1.08166487 & Up & 0.02824276 & 0.70952942 \\
\hline ACI06028.4-20I & 1.08382489 & Up & 0.04826811 & 0.75909133 \\
\hline Inc-LYSMD3-2:I & 1.0840707 & Up & 0.01190799 & 0.63705043 \\
\hline RNU6-709P-20I & 1.08652258 & Up & $0.004466 \mathrm{I}$ & 0.55695089 \\
\hline Inc-FAM75B-2:I & 1.08830209 & Up & 0.02084896 & 0.68520443 \\
\hline Inc-CDYL-9:I & 1.08948305 & Up & 0.00637373 & 0.55695089 \\
\hline Inc-PDEIC-2:3 & 1.09008387 & $U_{p}$ & 0.00078375 & 0.41823037 \\
\hline Inc-SRRMI-4:I & 1.09019086 & Up & 0.01620667 & 0.65476375 \\
\hline AC0I $2363.1-20 I$ & 1.09404463 & Up & 0.04399763 & 0.74634378 \\
\hline Inc-RPILI-3:3 & 1.09943997 & $U_{p}$ & 0.01034911 & 0.62720663 \\
\hline Inc-TBPL2-3:I & I.09976307 & Up & 0.02214589 & 0.6889443 \\
\hline AC09I078.I-20I & I.10348942 & Up & 0.01206545 & 0.63775317 \\
\hline AC079456.I-20I & I. 10454579 & Up & 0.00559852 & 0.55695089 \\
\hline Inc-ASPH-5:3 & I.10750927 & $U_{p}$ & 0.02233274 & $0.6901846 \mid$ \\
\hline Inc-EXTL3-I:3 & 1.11176621 & Up & 0.02864791 & 0.71004105 \\
\hline TMEMI08-ASI-20I & I.II243266 & Up & 0.04766525 & 0.75874445 \\
\hline LINC00463 & I.II30875 & $U_{p}$ & 0.02148542 & 0.6875777 \\
\hline TYMSOS-202 & I.II588444 & $U_{p}$ & 0.00210834 & 0.49624236 \\
\hline Inc-FAMI9A3-7:I & 1.11995024 & Up & $4.32 \times 10^{-5}$ & 0.23823842 \\
\hline AC0II48I.I-20I & I. 1228884 & Up & 0.02649529 & 0.7025969 \\
\hline ATRNLI & I.1236853I & Up & 0.01534705 & 0.65476375 \\
\hline SNHGI4-22I & 1.12569191 & Up & 0.04719373 & 0.75708084 \\
\hline Inc-ACAD I I-2:I & 1.12954103 & Up & 0.01867463 & 0.66684299 \\
\hline ACI 27496.3-20I & 1.13361155 & Up & 0.00180459 & 0.49597572 \\
\hline Inc-FAM27B-9:I & I.13529595 & Up & 0.01792398 & 0.65667837 \\
\hline Inc-ANO6-2:I & 1.14150763 & $U_{p}$ & 0.00932979 & 0.6151086 \\
\hline Inc-BAG4-4:I & I.14644342 & Up & 0.02936139 & 0.71263333 \\
\hline Inc-ABCAI-I:2 & I. 14734442 & Up & 0.00747609 & 0.58535254 \\
\hline Inc-GGPSI-7:I & 1.14849917 & Up & 0.01982258 & 0.68003654 \\
\hline Inc-ITPRIP-7:I & $1.1488 \mid 355$ & Up & 0.02032843 & 0.68302181 \\
\hline Inc-PCBPI-4:I & 1.15063821 & Up & 0.00625079 & 0.55695089 \\
\hline Inc-PABPC3-3:2 & 1.15120963 & Up & 0.00091832 & 0.41823037 \\
\hline Inc-HERC4-I:I & I.15522135 & Up & 0.03708868 & 0.73471054 \\
\hline Inc-IQCH-7:I & 1.1603186 & $U_{p}$ & 0.01001295 & 0.62174278 \\
\hline Inc-CI6orf78-5:3 & I.16644237 & $U_{p}$ & $8.81 \times 10^{-5}$ & 0.23823842 \\
\hline CIQTNF7-ASI-202 & 1.17053152 & Up & 0.00916598 & 0.6151086 \\
\hline Inc-VASH2-I:I & I. 17220568 & Up & 0.04245329 & 0.74199989 \\
\hline LINCOI255-204 & I.17230984 & Up & 0.00953825 & 0.6151086 \\
\hline Inc-ATG3-2:I & I.17235799 & $U_{p}$ & 0.01125376 & 0.63705043 \\
\hline Inc-GLTIDI-5:I & 1.17473163 & Up & 0.04455268 & $0.748594 \mid 3$ \\
\hline Inc-CR392000.I-2:I & I. 17483834 & Up & 0.04007513 & 0.74199989 \\
\hline Inc-HMGB2-5: I & I.17590206 & $U_{p}$ & 0.02536006 & 0.69966978 \\
\hline Inc-CDY2A-I3:I & 1.18188326 & $U_{p}$ & 0.01485835 & 0.65476375 \\
\hline
\end{tabular}

(Continued) 
Table 5 (Continued).

\begin{tabular}{|c|c|c|c|c|}
\hline IncRNAs & Log2FoldChange & Regulation & P-value & P-adj \\
\hline AC023355.I-202 & 1.1849183 & Up & 0.01732148 & 0.65667837 \\
\hline Inc-USPI 2-5:2 & I.18622738 & Up & 0.04358103 & 0.74483576 \\
\hline LOCI01929080 & I. 19054239 & Up & 0.01223231 & 0.63775317 \\
\hline Inc-KCNC2-5:I & 1.19486915 & Up & 0.009252 & 0.6151086 \\
\hline Inc-KNTCI-4:I & I.195359II & Up & 0.00297243 & 0.53593679 \\
\hline Inc-JAM3-8:I & I. 19852384 & Up & 0.00257337 & 0.51224337 \\
\hline Inc-NKX6-I-I0:I & 1.20120674 & Up & 0.01409262 & 0.64546217 \\
\hline Inc-ARG2-I:2 & 1.20386745 & Up & 0.00126408 & $0.43 I 52476$ \\
\hline Inc-KALRN-4:I & 1.20497602 & Up & 0.00582578 & 0.55695089 \\
\hline Inc-CORO2B-I:2 & 1.20602566 & $U_{p}$ & 0.00664972 & 0.56471029 \\
\hline Inc-URB2-I:3 & $1.207 \mid 15033$ & Up & 0.02185892 & 0.68799902 \\
\hline Inc-HS3ST3AI-5:2 & 1.21290644 & Up & 0.04894508 & 0.75985275 \\
\hline Z98885.3-20I & 1.21299164 & Up & 0.01621373 & 0.65476375 \\
\hline Inc-WDR64-2:2 & 1.21682833 & Up & 0.04578576 & 0.75240869 \\
\hline LOCI01929452 & 1.2233498 & Up & 0.03206412 & 0.72031743 \\
\hline Inc-PAICS-4:I & 1.22411726 & Up & 0.0006972 & 0.39679443 \\
\hline AL020994.I-20I & 1.2262244 & Up & 0.00037776 & 0.33998788 \\
\hline Inc-CNIH3-I:I & 1.2316038 & $U_{p}$ & 0.00010236 & 0.23823842 \\
\hline PRNCRI & I.240687| & Up & 0.00123666 & $0.43 I 52476$ \\
\hline AC024475.I-20I & $|.2454329|$ & Up & 0.02421639 & 0.69616967 \\
\hline Inc-DDX52-2:I & 1.24950582 & Up & 0.00022629 & 0.32373463 \\
\hline Inc-SLFNI 2-6:I & I.24993787 & $U_{p}$ & 0.03623298 & 0.73000405 \\
\hline AC093297.I-20I & 1.26032356 & Up & 0.03683869 & 0.73355985 \\
\hline UI.I8-20I & I.26323937 & Up & 0.00602542 & 0.55695089 \\
\hline Inc-GTPBP5-3:I & 1.26463283 & Up & 0.03100912 & 0.72031743 \\
\hline LINC02773-20I & 1.27552196 & Up & 0.00387626 & 0.55546359 \\
\hline Inc-FAM I 04A-4:2 & 1.2756097 & $U_{p}$ & 0.00884705 & 0.6151086 \\
\hline Inc-SYCP2-3:I & 1.27585656 & $U_{p}$ & 0.01741856 & 0.65667837 \\
\hline NONHSATI 06296 & 1.28232348 & Up & 0.02753193 & 0.70738927 \\
\hline Inc-TMEMI23-3:2 & $1.2843|2| 4$ & Up & 0.00636859 & 0.55695089 \\
\hline LOCI02723833 & I.28894382 & Up & 0.00193168 & 0.49624236 \\
\hline Inc-GPR |49-4:I & I.29256774 & Up & 0.00893933 & 0.6151086 \\
\hline Inc-SLCO4CI-4:I & 1.29343344 & Up & 0.02627738 & 0.7025969 \\
\hline AL35954I.I-20I & 1.2959487 & Up & 0.009658 & 0.6151086 \\
\hline ATP2BI-ASI-20I & $1.297669 \mid 8$ & Up & 0.00356845 & 0.54892347 \\
\hline AP000879.I-202 & I.29942575 & Up & 0.00578872 & 0.55695089 \\
\hline Inc-RP| I-6L6.2.I-3:I & 1.3032656 & Up & 0.01303033 & 0.63911018 \\
\hline LOCI02546298 & I.30753746 & Up & $0.000 \mid 4704$ & 0.27188326 \\
\hline Inc-ZCCHC9-2:5 & I.30773357 & Up & 0.01041044 & 0.62720663 \\
\hline Inc-ITGB I-4:2 & 1.31033236 & Up & 0.00767846 & 0.59393265 \\
\hline Inc-RPII-257K9.7.I-3:I & 1.31103839 & Up & 0.01633508 & 0.65588218 \\
\hline LINC02864-202 & 1.31194048 & Up & 0.01303027 & 0.63911018 \\
\hline LINC0I855-20I & I.32678547 & Up & 0.00081184 & 0.41823037 \\
\hline Inc-C8orf4-I:2 & 1.33018827 & Up & 0.00593828 & 0.55695089 \\
\hline Inc-RPII-I50OI2.3.I-I:4 & $1.340|765|$ & $U_{p}$ & 0.01509456 & 0.65476375 \\
\hline Inc-ZNF503-AS2-9:I & I.3448875| & Up & 0.03296829 & 0.7208645 \\
\hline ACI 20498.2-20I & 1.34578622 & Up & 0.00320335 & 0.53930742 \\
\hline Inc-HEATR4-5:I & $1.346 \mid 323$ & Up & 0.03623736 & 0.73000405 \\
\hline Inc-CNRI-I:2 & 1.35206876 & Up & 0.00627778 & 0.55695089 \\
\hline
\end{tabular}

(Continued) 
Table 5 (Continued).

\begin{tabular}{|c|c|c|c|c|}
\hline IncRNAs & Log2FoldChange & Regulation & P-value & P-adj \\
\hline Inc-SMYD5-I:4 & 1.35280066 & $U_{p}$ & 0.00601195 & 0.55695089 \\
\hline Inc-NUPLI-I:I & 1.35697674 & $U_{p}$ & 0.02392115 & 0.69616967 \\
\hline Inc-AKRIE2-5:I & 1.35847468 & $U_{p}$ & 0.01769903 & 0.65667837 \\
\hline ACOI23I4.2-20I & 1.35985353 & $U_{p}$ & 0.03496286 & 0.72695785 \\
\hline Inc-CNDP2-I:I & 1.36188928 & $U_{p}$ & 0.00584868 & 0.55695089 \\
\hline Inc-CHGA-2:I & 1.3642467 & $U_{p}$ & 0.02269448 & 0.69285761 \\
\hline MIRI307 & $|.3648424|$ & $U_{p}$ & $0.036 \mid 3377$ & 0.73000405 \\
\hline Inc-SRGN-3:I & $1.37|1729|$ & $U_{p}$ & 0.00300049 & 0.53721319 \\
\hline Inc-XRCC2-3:I & 1.38735139 & $U_{p}$ & 0.00200603 & 0.49624236 \\
\hline LRRK2-DT-202 & 1.39577039 & $U_{p}$ & 0.01113659 & 0.63705043 \\
\hline $\mathrm{ACI} / 3133 . \mathrm{I}-203$ & 1.43171942 & Up & 0.00455193 & 0.55695089 \\
\hline Inc-CCL3-I:2 & 1.43199469 & $U_{p}$ & 0.02692513 & 0.70508418 \\
\hline Inc-RTKN2-5:I & 1.43324395 & $U_{p}$ & 0.00204034 & 0.49624236 \\
\hline BX005019.I-201 & 1.43992263 & $U_{p}$ & 0.0338234 & 0.72437788 \\
\hline Inc-CI4orfl 82-4:I & 1.45445268 & $U_{p}$ & 0.01695345 & 0.65667837 \\
\hline AC034I99.I-20I & 1.47353584 & $U_{p}$ & 0.0213565 & 0.6875777 \\
\hline Inc-LYZLI-I0:I & 1.48259417 & Up & 0.00124885 & 0.43152476 \\
\hline Inc-LRIG I-3:I & 1.48289858 & $U_{p}$ & 0.00178858 & 0.49597572 \\
\hline AL39I24I.I-20I & 1.48399065 & $U_{p}$ & 0.03316116 & 0.72302337 \\
\hline Inc-MAPILC3B2-4:3 & 1.49320191 & $U_{p}$ & 0.00670515 & 0.56471029 \\
\hline Inc-RAD5IB-I:I & 1.49785989 & $U_{p}$ & 0.00014303 & 0.27188326 \\
\hline MIR425 & 1.50669826 & $U_{p}$ & 0.0042996 & 0.55695089 \\
\hline Inc-SIM2-2:I & 1.52947082 & $U_{p}$ & 0.04100787 & 0.74199989 \\
\hline PRMT5-ASI-206 & 1.53103618 & Up & $0.0085380 I$ & 0.6151086 \\
\hline AC0270I8.I-202 & 1.53360865 & $U_{p}$ & 0.01496409 & 0.65476375 \\
\hline Inc-ARF6-5:I & 1.5402516 & $U_{p}$ & 0.00426956 & 0.55695089 \\
\hline Inc-KCNK9-3:I & 1.55537316 & $U_{p}$ & $8.35 \times 10^{-5}$ & 0.23823842 \\
\hline LOC 102724484 & 1.55813648 & $U_{p}$ & 0.00484276 & 0.55695089 \\
\hline Inc-VAMP3-3:I & 1.55906933 & $U_{p}$ & 0.01857284 & 0.66521456 \\
\hline Inc-MRPSI8A-2:6 & 1.5595027 & $U_{p}$ & 0.00187188 & 0.49624236 \\
\hline Inc-TACR2-5:I & 1.56018317 & Up & 0.03954105 & 0.74199989 \\
\hline Inc-APCDDIL-I:I & 1.56781969 & $U_{p}$ & 0.00071636 & 0.3987193 \\
\hline Inc-RILP-I:5 & 1.57733865 & $U_{p}$ & 0.01232391 & 0.63775317 \\
\hline NONHSATI06835 & 1.58291652 & $U_{p}$ & 0.04113014 & 0.74199989 \\
\hline AC066595.I-20I & 1.59152878 & $U_{p}$ & 0.00607687 & 0.55695089 \\
\hline AP002505.2-20I & 1.65111587 & Up & 0.00528326 & 0.55695089 \\
\hline Inc-ROPNIB-8:I & 1.66748053 & $U_{p}$ & 0.0033198 & 0.54489032 \\
\hline Inc-NLK-I:2 & 1.66793517 & $U_{p}$ & 0.01702869 & 0.65667837 \\
\hline Inc-SH3BGRL2-8: I & $1.6825676 \mid$ & $U_{p}$ & 0.01627335 & 0.65588218 \\
\hline LINC00862 & 1.69076057 & $U_{p}$ & $0.0436085 I$ & 0.74483576 \\
\hline Inc-XK-2:I & 1.69608599 & $U_{p}$ & 0.02384559 & 0.69616967 \\
\hline AL606468.I-20I & 1.72966979 & $U_{p}$ & 0.00784283 & 0.59809568 \\
\hline Inc-DRD3-2:I & 1.73507434 & $U_{p}$ & 0.0002036 & 0.32373463 \\
\hline Inc-ASMT-7:I & I.77985488 & $U_{p}$ & 0.00534204 & 0.55695089 \\
\hline AC02200I.3-20I & 1.78553082 & $U_{p}$ & 0.01479698 & 0.65476375 \\
\hline AC090643.2-20I & 1.83684053 & $U_{p}$ & 0.00079596 & 0.41823037 \\
\hline NONHSATI 06837 & 1.84066898 & Up & 0.02058467 & 0.68445357 \\
\hline ACI03993.I-202 & $|.854| 423 \mid$ & $U_{p}$ & 0.00017245 & 0.29435292 \\
\hline
\end{tabular}

(Continued) 
Table 5 (Continued).

\begin{tabular}{|c|c|c|c|c|}
\hline IncRNAs & Log2FoldChange & Regulation & P-value & P-adj \\
\hline Inc-CPXM2-2:2 & 1.86775188 & Up & 0.01688355 & 0.65667837 \\
\hline SNHG2I & 1.90852825 & $U_{p}$ & 0.00532982 & 0.55695089 \\
\hline$A C I \mid 602 I . I-20 I$ & 1.93990875 & Up & 0.02097564 & 0.68579407 \\
\hline LOCI024672I7 & 1.9623212 & $U_{p}$ & 0.00440907 & 0.55695089 \\
\hline Inc-C2orf62-I:I & 1.96238874 & $U_{p}$ & 0.01728119 & 0.65667837 \\
\hline PELATON-20I & $2.00463 \mid 47$ & Up & 0.03488425 & 0.72695785 \\
\hline Inc-C5-I:I & 2.0585191 & $U_{p}$ & 0.01120735 & 0.63705043 \\
\hline Inc-PON2-3:I & 2.06777784 & $U_{p}$ & 0.02628724 & 0.7025969 \\
\hline Inc-IFNA2-2:I & 2.17522445 & $U_{p}$ & 0.0119433 & 0.63705043 \\
\hline AL359258.I-20I & 2.17725336 & $U_{p}$ & 0.01246482 & 0.63775317 \\
\hline Inc-CEACAM6-I:I & $2.22056 \mid 45$ & Up & 0.02321939 & 0.69449317 \\
\hline Inc-EVI2A-I:I & 2.25337956 & $U_{p}$ & 0.00361694 & 0.54892347 \\
\hline Inc-CXCL3-I:I & 2.28502109 & Up & 0.03936439 & 0.74199989 \\
\hline Inc-SRGN-4:I & 2.3926122 & $U_{p}$ & 0.00530242 & 0.55695089 \\
\hline
\end{tabular}

Table 6 Results of Gene Ontology (GO) Enrichment Analysis of Differentially Expressed IncRNAs

\begin{tabular}{|l|l|l|l|l|}
\hline Ontology & Term & Description & Gene Number & P-adj \\
\hline BP & GO:0042116 & Macrophage activation & 5 & 0.01572240 \\
BP & GO:0043030 & Regulation of macrophage activation & 4 & 0.01885761 \\
CC & GO:0005902 & Microvillus & 4 & 0.02267082 \\
\hline
\end{tabular}

Abbreviations: BP, biological process; CC, cellular component.

the cellular localization of hsa_circ_0002202 in THP1-derived macrophages. The results showed that hsa_circ_0002202 was mainly localized in the cytoplasm of THP-1-derived macrophage. Furthermore, we found that knockdown of hsa_circ_0002202 suppressed the IFN-I-induced inflammation, which was in line with the previous study. ${ }^{51}$ These findings suggested that hsa_circ_0002202 play an important role in T1DM, which will be verified by tissue sample experiments, cell lines experiments, and even animal model construction in future studies.

The miRNAs in the T1DM-related ceRNA regulatory network are also important regulators in T1DM. In this study, we found that hsa_circ_0002207 could sponge miR-487a-3p, miR-576-5p, miR-326, miR$186-5 \mathrm{p}$, and many other miRNAs. Among the miRNAs, miR-487a-3p is reported to be up-regulated in the PBMCs of T1DM patients. ${ }^{52}$ Increased expression of miR-326 was identified in $\mathrm{PBMSCs}^{53}$ and peripheral blood lymphocytes ${ }^{54}$ of patients with T1DM. Another study showed a decreased expression level of miR-326 in PBMCs of patients with T1DM. ${ }^{55}$ These same or opposite results may be related to the genetic or environmental factors of T1DM patients. Besides, some other miRNAs in the ceRNA network may be related to other autoimmune disorders, which have strong complications with T1DM. The pro-inflammatory cytokines promote the miR-455-3p expression and then cause the inflammatory $\beta$-cell failure. ${ }^{56} \mathrm{miR}$ 31-5p, miR-665, miR-326, miR-224 are associated with inflammatory bowel disease. ${ }^{56-59}$ We will also conduct verification and analysis in future experimental studies to obtain more in-depth and comprehensive scientific conclusions.

In the present study, the biological function and potential pathways of the DEcircRNAs and DElncRNAs were also analyzed. The enriched GO terms were mainly related to extracellular matrix 
A

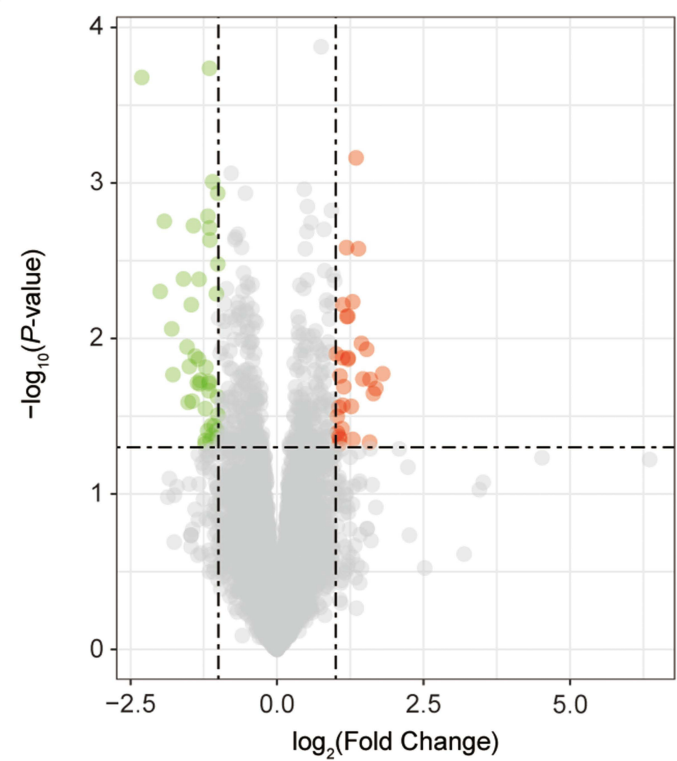

B

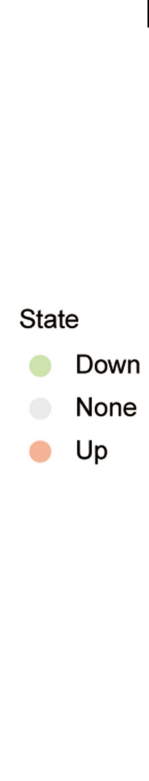

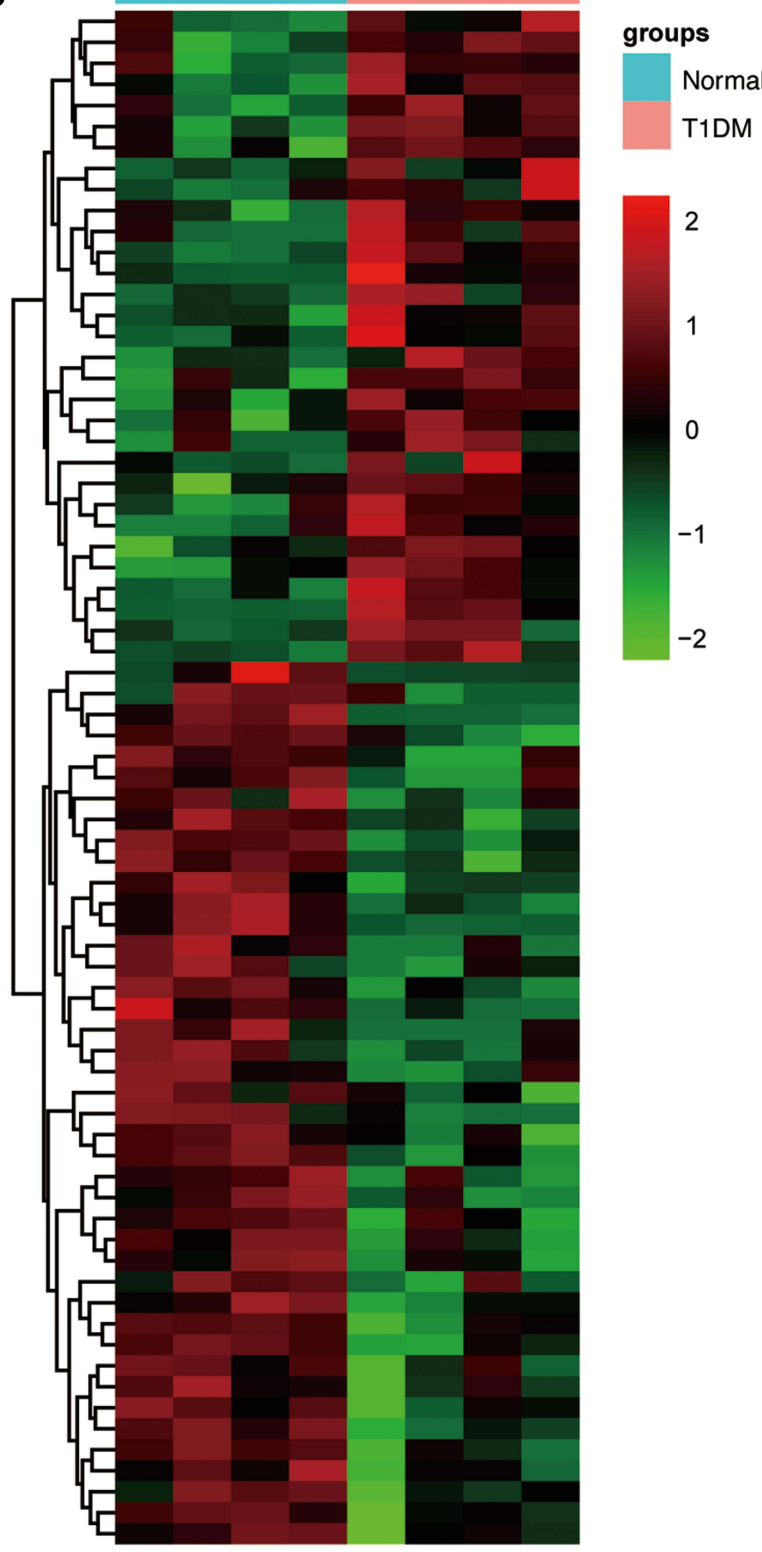

Figure 4 The differentially expressed mRNAs in TIDM and healthy patients. (A) Volcano plot of DEmRNAs between normal and TIDM groups. Red and green indicate upand downregulation, respectively. (B) Heatmap of DEmRNAs between normal and TIDM groups. Red color represents increased expression, and green color represents decreased expression. The darker the color, the greater the difference in mRNA expression. (C) Enrichment analysis of the DEmRNAs in the category molecular function.

components (ECM) and macrophage activation. The ECM contributes to islet inflammation and creates a permissive environment for immune cells to infiltrate the pancreatic islets and impair $\beta$-cell survival. ${ }^{60}$ Macrophages participate in interacting, directing, or restricting trafficking of the autoreactive-specific $\mathrm{T}$ cells into the islets via the IFN-I signaling during the development of T1DM. ${ }^{61}$ Thus, our in vitro experiments focused on macrophage inflammation, and the results indicated the anti-inflammatory effect of hsa circ_0002202 inhibition. In terms of the KEGG pathway, the protein digestion and absorption were enriched, indicating that the activation of these genes was closely related to the T1DM progression. 
Table 7 Differentially Expressed mRNAs Between the Control Group and TIDM Group

\begin{tabular}{|c|c|c|c|c|}
\hline mRNAs & Log2FoldChange & Regulation & P-value & P-adj \\
\hline RBFOXI & $-2.3079|2|$ & Down & 0.00020929 & 0.48868466 \\
\hline FAMI32B & -1.9940805 & Down & 0.00498156 & 0.78986357 \\
\hline SLFNLI & -1.9223862 & Down & 0.00176092 & 0.71338425 \\
\hline CLDN22 & -1.7965769 & Down & 0.00866828 & 0.8200064 \\
\hline GNAL & -1.7755788 & Down & 0.01707197 & 0.82671544 \\
\hline MUCI5 & -1.5965074 & Down & 0.00413517 & 0.78986357 \\
\hline CA8 & -1.5357652 & Down & 0.01131812 & 0.82671544 \\
\hline PTGDR & $-1.5|6536|$ & Down & 0.0257405 I & 0.82671544 \\
\hline TEXI3A & -1.495935 & Down & 0.01513717 & 0.82671544 \\
\hline TAAR2 & -1.4635903 & Down & 0.00606045 & 0.78986357 \\
\hline KIR3DLI & -1.4479969 & Down & 0.02527008 & 0.82671544 \\
\hline COL6A3 & -1.4263206 & Down & 0.0018801 & 0.71338425 \\
\hline B4GALNT2 & -1.3947065 & Down & 0.01298325 & 0.82671544 \\
\hline PARD3 & -1.3582746 & Down & 0.01926125 & $0.8267 \mid 544$ \\
\hline SORBSI & -1.341606 & Down & 0.01356421 & 0.82671544 \\
\hline GPR32 & -1.3306212 & Down & 0.0041603 & 0.78986357 \\
\hline DUOX2 & -1.3208459 & Down & 0.01957626 & 0.82671544 \\
\hline HPGD & -1.3088539 & Down & 0.01853011 & 0.82671544 \\
\hline NNMT & -1.2274537 & Down & 0.04763409 & 0.82671544 \\
\hline TMEM74 & $-1.225520 \mid$ & Down & 0.04530755 & 0.82671544 \\
\hline COL6A2 & -1.2224407 & Down & 0.02822533 & 0.82671544 \\
\hline HSPB8 & -1.2153355 & Down & 0.01531611 & 0.82671544 \\
\hline BFSPI & -1.1831939 & Down & 0.03922896 & 0.82671544 \\
\hline GNGII & -1.179029 & Down & 0.00163335 & 0.71338425 \\
\hline GPRI & -1.1631327 & Down & 0.02166454 & $0.8267 \mid 544$ \\
\hline B3GALT5 & -1.1584697 & Down & $0.0194624 \mathrm{I}$ & $0.8267 / 544$ \\
\hline C20orfI 73 & -1.1568903 & Down & 0.01892708 & $0.8267 / 544$ \\
\hline CYPIIBI & -1.152487 & Down & 0.00018277 & 0.48868466 \\
\hline $\mathrm{DHH}$ & -1.1517769 & Down & 0.00194518 & 0.71338425 \\
\hline CRYM & -1.1448803 & Down & 0.00232428 & 0.71338425 \\
\hline SMKRI & -1.138156 & Down & 0.04519693 & 0.82671544 \\
\hline BPIFB3 & -1.1194083 & Down & 0.03620019 & 0.82671544 \\
\hline FAMI50B & -1.1105272 & Down & 0.04200652 & $0.8267 / 544$ \\
\hline TEXII & -1.0988657 & Down & 0.00098015 & 0.71338425 \\
\hline NPR3 & -1.0702284 & Down & 0.03640359 & $0.8267 / 544$ \\
\hline ATPI3A4 & -1.0357916 & Down & 0.04369288 & 0.82671544 \\
\hline CAMK2N2 & -1.0343627 & Down & 0.00515457 & 0.78986357 \\
\hline KIR2DL3 & -1.0313509 & Down & 0.03893691 & $0.8267 / 544$ \\
\hline CACNA2D2 & $-1.015540 \mid$ & Down & 0.02373916 & $0.8267 / 544$ \\
\hline WDR76 & -1.0120059 & Down & 0.00116238 & 0.71338425 \\
\hline GPRI 80 & -1.0109237 & Down & 0.00332039 & 0.78986357 \\
\hline AMHR2 & $-|.008992|$ & Down & 0.03106529 & 0.82671544 \\
\hline PCDHBI5 & I.0075442। & $U_{p}$ & 0.01257116 & $0.8267 / 544$ \\
\hline MARCKS & $1.0270 \mid 386$ & $U_{p}$ & 0.03176063 & $0.8267 \mid 544$ \\
\hline DEFBI 23 & 1.02969089 & Up & 0.04036705 & $0.8267 / 544$ \\
\hline DNAJB7 & I.05742045 & Up & 0.04277946 & $0.8267 / 544$ \\
\hline C2CD4B & I.0577298 & Up & 0.02772476 & 0.82671544 \\
\hline IL6 & I.05994453 & Up & 0.04788638 & 0.82671544 \\
\hline OR2LI3 & 1.07004067 & $U_{p}$ & 0.04427013 & 0.82671544 \\
\hline
\end{tabular}

(Continued) 
Table 7 (Continued).

\begin{tabular}{|c|c|c|c|c|}
\hline mRNAs & Log2FoldChange & Regulation & P-value & P-adj \\
\hline Cl7orf64 & 1.07345437 & $U_{p}$ & 0.01738992 & 0.82671544 \\
\hline SGKI & I.10667874 & $U_{p}$ & 0.03799544 & 0.82671544 \\
\hline COMP & 1.11621286 & Up & 0.01328963 & 0.82671544 \\
\hline RASSF8 & 1.1211237 & $U_{p}$ & 0.0268822 & 0.82671544 \\
\hline SLC6AI4 & I.12250998 & $U_{p}$ & 0.00605879 & 0.78986357 \\
\hline RAPGEF5 & I.13847388 & Up & 0.02042072 & 0.82671544 \\
\hline GREM2 & I.18378203 & $U_{p}$ & 0.00260009 & 0.71338425 \\
\hline ARRDC3 & I.18980995 & $U_{p}$ & 0.00721663 & 0.78986357 \\
\hline FILIPIL & 1.20891948 & $U_{p}$ & 0.00719201 & 0.78986357 \\
\hline ADGRG4 & I.20976755 & $U_{p}$ & 0.01358302 & 0.82671544 \\
\hline ASIC2 & 1.21384452 & $U_{p}$ & 0.01333649 & 0.82671544 \\
\hline MARCHI & 1.26722335 & Up & 0.02731178 & 0.82671544 \\
\hline AGXT & 1.28961174 & Up & 0.00580496 & 0.78986357 \\
\hline HSPAI $2 A$ & 1.29400344 & $U_{p}$ & 0.04457166 & 0.82671544 \\
\hline C9orfl52 & 1.34773511 & $U_{p}$ & 0.00068838 & 0.71338425 \\
\hline MAPT & 1.38818206 & $U_{p}$ & 0.00264472 & 0.71338425 \\
\hline DOCK 4 & 1.43931041 & Up & 0.01074391 & 0.82671544 \\
\hline SKORI & 1.46453595 & Up & 0.01818479 & 0.82671544 \\
\hline CXCLI3 & 1.52896795 & $U_{p}$ & 0.01173074 & $0.8267 \mid 544$ \\
\hline SLC5A7 & I.5790437| & $U_{p}$ & $0.0464531 I$ & 0.82671544 \\
\hline LOC388780 & I.58723305 & $U_{p}$ & 0.01831872 & $0.8267 \mid 544$ \\
\hline CCL8 & I.643|4463 & Up & 0.02259273 & 0.82671544 \\
\hline PDK4 & I.68428709 & $U_{p}$ & 0.02094083 & 0.82671544 \\
\hline MYO7B & 1.80214766 & $U_{p}$ & 0.01684902 & $0.8267 \mid 544$ \\
\hline
\end{tabular}

Table 8 Results of Gene Ontology (GO) Enrichment Analysis of Differentially Expressed mRNAs

\begin{tabular}{|l|l|l|l|l|}
\hline Ontology & Term & Description & Gene Number & P-adj \\
\hline MF & GO:0004955 & Prostaglandin receptor activity & 2 & 0.043054 \\
MF & GO:0004954 & Prostanoid receptor activity & 2 & 0.043054 \\
MF & GO:0036I22 & BMP binding & 2 & 0.04305384 \\
\hline
\end{tabular}

Abbreviation: MF, molecular function.

We also identified some genes associated with T1DM, including GREM2, RAPGEF5, C9orf152, SMKR1, SORBS1, HSPB8, CACNA2D2, SKOR1, and SLFNL1. Among these, SORBS1 and CACNA2D2 are previously reported to be related to diabetes. SORBS1, a human homologue for c-Cbl-associated protein (CAP), is an important adaptor protein in the insulin-signaling pathway, and its genetic polymorphism is related to insulin resistance. $^{62}$
Meanwhile, another study showed that SORBS1 might be a new susceptibility gene for diabetic nephropathy. ${ }^{63}$ CACNA2D2 is the voltage-gated calcium channel auxiliary subunit alpha2delta2 gene that encodes a calcium channel protein, ${ }^{64}$ and Huang et al identified CACNA2D2 as a diabetes-related atherogenesis gene. ${ }^{65}$ Besides, GREM2 and HSPB8 are closely related to inflammatory response, ${ }^{66,67}$ suggesting a regulatory role in the T1DM macrophages inflammation. These 


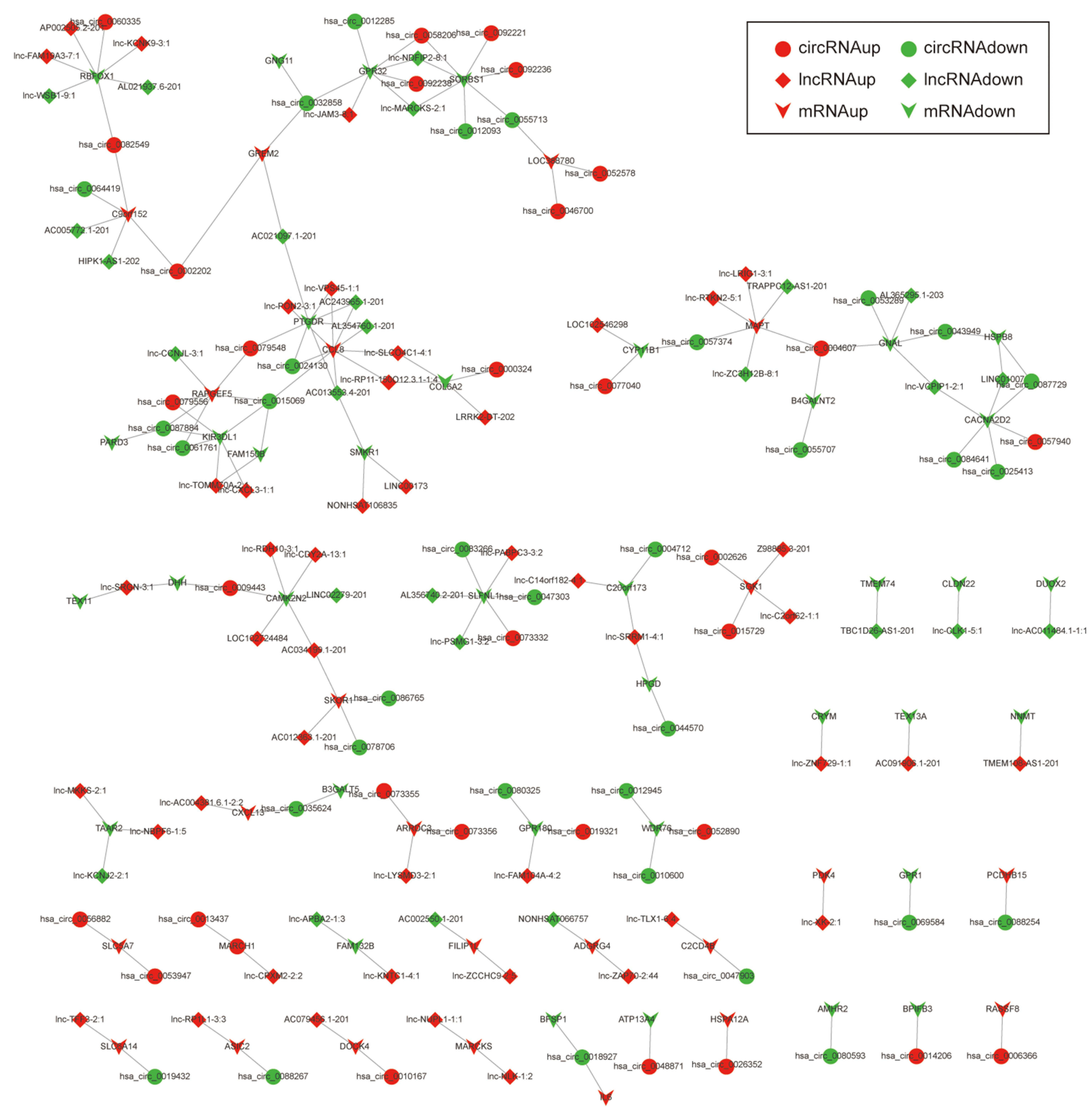

Figure 5 The circRNA-IncRNA-mRNA network. The round nodes represent DEcircRNAs, the rhombic nodes represent DElncRNAs, the v-type nodes represent DEmRNAs. The up or down-regulated genes are represented in red and green, respectively.

genes with special functions may be the latest molecular markers of T1DM and need further experimental verification.

Despite the above results, there are still some limitations in the current study. First, the small sample size may affect the number of altered RNAs. Further studies based on a larger sample size should be performed to confirm our conclusions. Second, besides acting as miRNA sponges, circRNAs or lncRNAs can also regulate gene expression by affecting transcription. ${ }^{68,69}$ At the same time, most of the results are obtained using bioinformatics analysis. Thus, experimental studies are needed to validate our results and further investigate the deeper mechanism. Third, the progress of T1DM is not 
Table 9 The Fold Change of All miRNAs Between the Control Group and TIDM Group

\begin{tabular}{|c|c|c|c|c|}
\hline miRNAs & Log2FoldChange & Regulation & P-value & P-adj \\
\hline miR-I-3p & -1.5165809 & Down & 0.00670577 & 0.40231598 \\
\hline miR-I33b & $-|.285048|$ & Down & 0.00702946 & 0.40231598 \\
\hline miR-99b-5p & -0.8676123 & Down & 0.03302164 & 0.59871978 \\
\hline let-7e-5p & -0.7792672 & Down & 0.03530638 & $0.5987 \mid 978$ \\
\hline$m i R-125 a-5 p$ & -0.7761986 & Down & 0.05533304 & 0.64056718 \\
\hline miR-6885-5p & -0.637504 & Down & $1.38 \times 10^{-6}$ & 0.00354783 \\
\hline$m i R-55 I b-3 p$ & $-0.5977 \mid 45$ & Down & 0.01706182 & 0.53220869 \\
\hline miR-10a-5p & -0.5042807 & Down & 0.04874767 & $0.6141250 \mid$ \\
\hline$m i R-181 a-2-3 p$ & -0.4754952 & Down & 0.00897879 & 0.40696367 \\
\hline miR-27b-3p & -0.4519303 & Down & 0.07953434 & 0.68910078 \\
\hline$m i R-15 \mid a-3 p$ & -0.4402091 & Down & 0.02080807 & 0.53734625 \\
\hline miR-139-5p & $-0.438 \mid 705$ & Down & 0.04665277 & $0.614|250|$ \\
\hline$m i R-I 5 I b$ & -0.4337404 & Down & 0.00166047 & 0.32405862 \\
\hline miR-584-5p & -0.4115123 & Down & $0.2422695 I$ & 0.80715125 \\
\hline miR-15Ia-5p & -0.4054726 & Down & 0.00857359 & 0.40696367 \\
\hline miR-5787 & -0.4016736 & Down & 0.00690767 & 0.40231598 \\
\hline miR-130a-3p & -0.3983994 & Down & $0.065|842|$ & 0.65598378 \\
\hline miR-133a-3p & -0.3784617 & Down & 0.01526582 & 0.49284222 \\
\hline$m i R-43 I-5 p$ & -0.376715 & Down & 0.26619136 & 0.82438552 \\
\hline miR-30a-5p & -0.3686175 & Down & 0.04189063 & 0.60118096 \\
\hline miR-23b-3p & -0.3650878 & Down & 0.06512818 & 0.65598378 \\
\hline miR-598-3p & -0.354844 I & Down & 0.00450404 & 0.34045223 \\
\hline miR-98-5p & $-0.35 \mid 2228$ & Down & 0.02419159 & 0.55019809 \\
\hline miR-126-5p & $-0.34607 / 3$ & Down & 0.10065779 & 0.73085631 \\
\hline$m i R-3120-3 p$ & -0.3425895 & Down & 0.00372022 & 0.34045223 \\
\hline miR-330-3p & -0.3418255 & Down & 0.04863712 & 0.61412501 \\
\hline miR-6085 & -0.3367 & Down & 0.01118897 & 0.43937422 \\
\hline miR-196b-5p & $-0.335288 \mathrm{I}$ & Down & 0.05202185 & 0.63253996 \\
\hline miR-324-5p & -0.3283337 & Down & 0.0599078 I & 0.64832335 \\
\hline miR-210-3p & -0.3265283 & Down & 0.30730975 & 0.84469096 \\
\hline miR-326 & -0.3226899 & Down & 0.04104522 & 0.60118096 \\
\hline miR-18Id-5p & -0.3203042 & Down & 0.04674485 & $0.614|250|$ \\
\hline miR-335-5p & -0.2992288 & Down & 0.28050619 & $0.8310854 \mid$ \\
\hline miR-5739 & $-0.298|96|$ & Down & 0.00422458 & 0.34045223 \\
\hline miR-126-3p & -0.293697 & Down & 0.0543304 I & 0.64056718 \\
\hline miR-590-5p & -0.2899434 & Down & 0.04821654 & $0.614|250|$ \\
\hline$m i R-146 a-5 p$ & -0.2839604 & Down & 0.1538926 & 0.75393664 \\
\hline$m i R-148 a-3 p$ & -0.28039 & Down & 0.23451265 & 0.80105138 \\
\hline$m i R-6756-5 p$ & -0.2760184 & Down & 0.00400347 & 0.34045223 \\
\hline miR-199a-3p & -0.2669967 & Down & 0.18245092 & $0.7735088 \mathrm{I}$ \\
\hline miR-28-5p & -0.2650704 & Down & 0.00020694 & 0.11527458 \\
\hline miR-128-3p & -0.2599197 & Down & 0.04105635 & 0.60118096 \\
\hline$m i R-301 a-3 p$ & -0.2587877 & Down & 0.1947074 & 0.7799749 | \\
\hline miR-652-3p & $-0.257 \mid 135$ & Down & 0.014974 & 0.49284222 \\
\hline$m i R-130 \mid-3 p$ & $-0.25575 \mathrm{II}$ & Down & 0.01179643 & 0.43937422 \\
\hline miR-379-5p & -0.2531956 & Down & 0.355301 & 0.8588192 \\
\hline$m i R-18 \mid c-5 p$ & -0.2521543 & Down & 0.13770206 & 0.75376261 \\
\hline miR-432-5p & -0.2503287 & Down & 0.48408203 & 0.90449982 \\
\hline miR-18b-5p & -0.2457859 & Down & 0.00032042 & $0.1|76394|$ \\
\hline miR-194-5p & -0.2451756 & Down & $0.22699|3|$ & $0.79|0224|$ \\
\hline
\end{tabular}

(Continued) 
Table 9 (Continued).

\begin{tabular}{|c|c|c|c|c|}
\hline miRNAs & Log2FoldChange & Regulation & P-value & P-adj \\
\hline miR-127-3p & -0.2446309 & Down & 0.33136688 & 0.85489649 \\
\hline miR-223-3p & -0.2440992 & Down & 0.01055265 & 0.43937422 \\
\hline miR-32-5p & -0.2428206 & Down & 0.0475609 & $0.6|4| 250 \mid$ \\
\hline miR-328-3p & -0.2426408 & Down & 0.04244989 & 0.60118096 \\
\hline miR-132-3p & -0.2426316 & Down & 0.07861796 & 0.68910078 \\
\hline let-7f-5p & $-0.242570 \mathrm{I}$ & Down & 0.01112698 & 0.43937422 \\
\hline$m i R-493-5 p$ & -0.2404954 & Down & $0.29956|9|$ & 0.83872374 \\
\hline miR-27a-3p & -0.2375163 & Down & 0.07375852 & 0.68406327 \\
\hline miR-I307-5p & -0.2345506 & Down & $0.048377 \mid 4$ & $0.614|250|$ \\
\hline$m i R-495-3 p$ & -0.2335808 & Down & 0.44444021 & 0.88952595 \\
\hline miR-6I65 & -0.2328824 & Down & 0.04757592 & $0.614|250|$ \\
\hline$m i R-22 I-3 p$ & -0.2320004 & Down & 0.19653159 & 0.7799749 I \\
\hline miR-43I7 & -0.2301339 & Down & $9.82 \times 10^{-5}$ & 0.08408842 \\
\hline miR-374c-5p & -0.2292803 & Down & 0.02737626 & $0.58|4627|$ \\
\hline miR-152-3p & $-0.2286|8|$ & Down & 0.06717209 & 0.65890175 \\
\hline$m i R-497-5 p$ & -0.2266935 & Down & 0.08775824 & $0.7109058 \mathrm{I}$ \\
\hline miR-195-5p & $-0.2257 \mid 44$ & Down & $0.1|86795|$ & 0.74226488 \\
\hline miR-744-5p & -0.2244425 & Down & 0.11526467 & 0.74226488 \\
\hline miR-543 & $-0.216885 \mid$ & Down & 0.13054129 & 0.74892227 \\
\hline miR-7-I-3p & -0.2147568 & Down & 0.00184957 & 0.32405862 \\
\hline miR-I7-3p & $-0.2|2603|$ & Down & 0.07709504 & 0.68910078 \\
\hline miR-22-5p & -0.2119735 & Down & 0.00065497 & 0.15302397 \\
\hline miR-6073 & -0.2022809 & Down & 0.47456765 & 0.8954764 \\
\hline$m i R-26 a-5 p$ & -0.2017921 & Down & 0.03685477 & 0.59871978 \\
\hline miR-148b-3p & -0.2014556 & Down & 0.27332485 & 0.83107769 \\
\hline miR-22-3p & -0.2014406 & Down & 0.00809954 & 0.40696367 \\
\hline miR-136-5p & -0.1997377 & Down & 0.06492627 & 0.65598378 \\
\hline miR-376c-3p & -0.1996448 & Down & 0.64319632 & $0.952746 \mid 4$ \\
\hline miR-199a-5p & -0.1994898 & Down & $0.359 \mid 2007$ & 0.8588192 \\
\hline$m i R-340-5 p$ & -0.198198 & Down & 0.28288034 & 0.83193599 \\
\hline miR-769-5p & -0.1956911 & Down & $0.2258 \mid 488$ & $0.79|0224|$ \\
\hline miR-I27I-5p & -0.1944416 & Down & 0.19561173 & 0.7799749 I \\
\hline miR-224-5p & -0.1934675 & Down & 0.68365226 & 0.95853044 \\
\hline miR-6852-5p & -0.191647 & Down & 0.00589685 & $0.3885875 \mathrm{I}$ \\
\hline miR-337-5p & -0.1901206 & Down & 0.36210628 & 0.8588192 \\
\hline$m i R-625-5 p$ & -0.1893543 & Down & 0.54798536 & 0.92261663 \\
\hline miR-376a-3p & -0.1877679 & Down & $0.650 \mid 4785$ & 0.95321673 \\
\hline miR-10I-3p & -0.1823045 & Down & 0.36292288 & 0.8588192 \\
\hline miR-487a-3p & -0.1822157 & Down & 0.16729778 & $0.769|508|$ \\
\hline$m i R-4 \mid I-5 p$ & -0.1820523 & Down & 0.11624338 & 0.74226488 \\
\hline miR-33a-5p & -0.1803366 & Down & 0.1091139 & 0.74226488 \\
\hline miR-2355-5p & -0.1784709 & Down & 0.01806897 & 0.53734625 \\
\hline miR-182-5p & -0.1775695 & Down & 0.00022427 & 0.11527458 \\
\hline miR-42I & $-0.177 \mid 404$ & Down & 0.03728568 & $0.5987 / 978$ \\
\hline miR-339-5p & -0.1761355 & Down & 0.1359837 & 0.75376261 \\
\hline miR-186-5p & $-0.1734 \mid 46$ & Down & 0.14060276 & $0.7537626 \mathrm{I}$ \\
\hline miR-10b-5p & -0.1714752 & Down & 0.00027399 & 0.11735825 \\
\hline miR-5584-3p & -0.1686341 & Down & 0.01165649 & 0.43937422 \\
\hline$m i R-215-5 p$ & -0.1682987 & Down & $0.3002365 I$ & 0.83872374 \\
\hline
\end{tabular}

(Continued) 
Table 9 (Continued).

\begin{tabular}{|c|c|c|c|c|}
\hline miRNAs & Log2FoldChange & Regulation & P-value & P-adj \\
\hline $\mathrm{miR}-320 \mathrm{c}$ & -0.1660735 & Down & 0.14388218 & 0.75376261 \\
\hline miR-I54-3p & -0.1638598 & Down & 0.20356636 & 0.77997491 \\
\hline$m i R-30 b-5 p$ & -0.1626831 & Down & 0.00529837 & 0.36854655 \\
\hline miR-18a-5p & -0.1601252 & Down & $0.3050349 \mid$ & 0.83933588 \\
\hline miR-505-5p & -0.159129 & Down & 0.03015598 & 0.59616058 \\
\hline miR-I46b-5p & -0.1575493 & Down & 0.29357044 & 0.83872374 \\
\hline miR-339-3p & -0.1564803 & Down & 0.09255755 & $0.7228707 \mid$ \\
\hline miR-382-5p & -0.1556784 & Down & 0.57309052 & 0.93390911 \\
\hline miR-24-I-5p & -0.1555473 & Down & 0.0214993 & 0.53734625 \\
\hline let-7g-5p & -0.1552145 & Down & 0.0589026 & 0.64832335 \\
\hline$m i R-|4|-3 p$ & -0.1552026 & Down & 0.2682486 & 0.82860446 \\
\hline$m i R-4 \mid 0-3 p$ & -0.1546221 & Down & 0.67560231 & 0.95648223 \\
\hline miR-4484 & -0.1524305 & Down & 0.15907323 & 0.75988515 \\
\hline miR-29c-3p & -0.1520516 & Down & 0.27357655 & 0.83107769 \\
\hline miR-I43-3p & -0.1517947 & Down & $0.5724,9896$ & 0.93390911 \\
\hline let-7a-5p & -0.1495243 & Down & 0.03567474 & $0.5987 / 978$ \\
\hline$m i R-185-5 p$ & -0.1490736 & Down & 0.02508744 & 0.55568816 \\
\hline miR-320e & -0.1469298 & Down & 0.28061814 & $0.8310854 I$ \\
\hline miR-I 202 & -0.1447347 & Down & 0.21813416 & 0.78840757 \\
\hline miR-340-3p & -0.143247 & Down & 0.33859524 & 0.85489649 \\
\hline miR-33I-3p & $-0.1428 \mid 54$ & Down & 0.14940403 & 0.75393664 \\
\hline$m i R-5196-5 p$ & -0.1427908 & Down & 0.00386774 & 0.34045223 \\
\hline$m i R-20 a-3 p$ & -0.142275 & Down & 0.0577224 & 0.64832335 \\
\hline$m i R-628-5 p$ & -0.1421981 & Down & 0.13722984 & 0.75376261 \\
\hline miR-4443 & -0.1404353 & Down & 0.3747999 & $0.8649907 \mid$ \\
\hline miR-38I-3p & -0.1404195 & Down & 0.62104792 & 0.94829596 \\
\hline$m i R-628-3 p$ & $-0.140085 \mid$ & Down & 0.16633836 & 0.76915081 \\
\hline$m i R-22 I-5 p$ & -0.1390408 & Down & 0.13217561 & 0.74892227 \\
\hline miR-429I & $-0.137|73|$ & Down & 0.22098446 & 0.79098893 \\
\hline miR-4323 & -0.1368585 & Down & 0.31730879 & 0.84917098 \\
\hline$m i R-30 e-3 p$ & -0.136426 & Down & 0.09755372 & 0.73085631 \\
\hline$m i R-29 c-5 p$ & -0.1359778 & Down & $0.16497 \mid 45$ & 0.76915081 \\
\hline miR-409-5p & -0.1346939 & Down & 0.30048022 & 0.83872374 \\
\hline miR-200b-3p & -0.1335934 & Down & 0.19960532 & 0.77997491 \\
\hline miR-15b-5p & -0.133299 & Down & $0.1460444 \mid$ & 0.75376261 \\
\hline$m i R-6763-5 p$ & -0.1325476 & Down & 0.05511816 & 0.64056718 \\
\hline miR-200c-3p & -0.1319846 & Down & 0.1147884 & 0.74226488 \\
\hline$m i R-652-5 p$ & -0.131862 & Down & 0.03226253 & $0.5987 / 978$ \\
\hline miR-103a-3p & -0.130681 & Down & 0.15260836 & 0.75393664 \\
\hline miR-548am-5p & -0.1286466 & Down & 0.14518387 & 0.75376261 \\
\hline miR-192-5p & -0.1269884 & Down & 0.43987942 & 0.88952595 \\
\hline miR-I07 & -0.1264259 & Down & 0.17675208 & 0.77062416 \\
\hline miR-29b-3p & -0.1262268 & Down & 0.44560284 & 0.88952595 \\
\hline$m i R-548 u$ & -0.1254266 & Down & 0.00389978 & 0.34045223 \\
\hline miR-18Ic-3p & -0.1246398 & Down & 0.38653847 & $0.8696 / 208$ \\
\hline$m i R-130 b-3 p$ & -0.1231865 & Down & 0.2852138 & 0.83289761 \\
\hline miR-320d & -0.123168 & Down & 0.24893553 & 0.80715125 \\
\hline miR-4788 & -0.1222474 & Down & 0.07291182 & 0.68406327 \\
\hline miR-484 & -0.1219113 & Down & 0.12894549 & 0.74892227 \\
\hline
\end{tabular}

(Continued) 
Table 9 (Continued).

\begin{tabular}{|c|c|c|c|c|}
\hline miRNAs & Log2FoldChange & Regulation & P-value & P-adj \\
\hline miR-323a-3p & -0.1208658 & Down & $0.268 \mid 7005$ & 0.82860446 \\
\hline miR-505-3p & -0.1202214 & Down & 0.3035112 & 0.83872374 \\
\hline miR-4749-3p & -0.1199575 & Down & 0.15869565 & 0.75988515 \\
\hline miR-I5a-3p & -0.1183105 & Down & 0.03708407 & $0.5987 / 978$ \\
\hline miR-320a & -0.1182161 & Down & 0.30403967 & 0.83872374 \\
\hline miR-642b-5p & -0.1180559 & Down & 0.1856993 & 0.77677007 \\
\hline miR-607I & -0.1133686 & Down & 0.00487931 & 0.35828046 \\
\hline miR-17-5p & -0.1130513 & Down & 0.37019062 & 0.8625475 \\
\hline miR-I307-3p & -0.1127969 & Down & 0.05488986 & 0.64056718 \\
\hline miR-I8Ia-3p & -0.1127909 & Down & 0.56807995 & 0.9303065 \\
\hline miR-363-3p & -0.1111045 & Down & 0.60447515 & 0.94188142 \\
\hline miR-6778-5p & -0.1085699 & Down & 0.1468648 & $0.7537626 \mathrm{I}$ \\
\hline miR-6766-3p & -0.1081158 & Down & 0.11137142 & 0.74226488 \\
\hline miR-370-3p & -0.1079054 & Down & 0.248405 I 4 & $0.807|5| 25$ \\
\hline miR-39|2-5p & -0.1077988 & Down & 0.11473463 & 0.74226488 \\
\hline miR-374a-5p & -0.107496 & Down & 0.38675744 & $0.8696 \mid 208$ \\
\hline miR-548as-3p & -0.1065386 & Down & 0.00778767 & 0.40696367 \\
\hline$m i R-627-5 p$ & -0.1061354 & Down & 0.02263304 & 0.53734625 \\
\hline miR-625-3p & -0.1049602 & Down & 0.16316836 & 0.76802692 \\
\hline miR-766-3p & -0.1048801 & Down & 0.39499968 & $0.873|074|$ \\
\hline miR-362-3p & -0.1026706 & Down & 0.45364747 & 0.89093879 \\
\hline miR-487b-3p & -0.1012303 & Down & 0.74956338 & 0.97742837 \\
\hline miR-95-3p & -0.1007765 & Down & 0.35316418 & 0.8588192 \\
\hline$m i R-545-3 p$ & -0.1005441 & Down & 0.06176618 & 0.65102084 \\
\hline$m i R-423-3 p$ & -0.0987889 & Down & $0.38|9455|$ & $0.866 \mid 2247$ \\
\hline miR-19b-3p & -0.0986038 & Down & 0.3747445 I & 0.8649907 I \\
\hline miR-548aj-3p & -0.0984553 & Down & 0.02308954 & 0.53734625 \\
\hline miR-6724-5p & -0.0973248 & Down & 0.05694408 & 0.64832335 \\
\hline miR-I 299 & -0.097018 & Down & 0.09632474 & 0.7308563 I \\
\hline miR-I306-5p & -0.0968363 & Down & 0.10062764 & 0.73085631 \\
\hline miR-302a-3p & -0.0965015 & Down & $0.026 \mid 2879$ & 0.55959148 \\
\hline miR-376b-3p & -0.096174 & Down & 0.33797387 & 0.85489649 \\
\hline miR-39I6 & -0.0958997 & Down & 0.01268218 & 0.45268334 \\
\hline miR-I287-5p & $-0.0954 \mid 45$ & Down & 0.06349926 & 0.65539395 \\
\hline miR-374b-5p & -0.0947825 & Down & 0.09792616 & 0.7308563 I \\
\hline miR-3I-5p & -0.0934179 & Down & $0.81 \mid 29553$ & 0.99064883 \\
\hline miR-4422 & -0.0933521 & Down & 0.07548529 & 0.68726073 \\
\hline miR-4682 & -0.0928982 & Down & 0.12060406 & 0.74226488 \\
\hline miR-3680-3p & -0.0922184 & Down & 0.00244716 & 0.34045223 \\
\hline miR-19I-5p & -0.0910243 & Down & 0.12444248 & 0.74454632 \\
\hline$m i R-36 I-3 p$ & -0.090964 & Down & 0.52487295 & 0.91825968 \\
\hline miR-26b-5p & -0.0908946 & Down & 0.21108356 & 0.78263372 \\
\hline miR-6727-5p & $-0.09082 \mid 4$ & Down & 0.0219247 & 0.53734625 \\
\hline miR-6888-3p & $-0.0908 \mid 22$ & Down & 0.02132992 & 0.53734625 \\
\hline $\mathrm{miR}-320 \mathrm{~b}$ & -0.0903495 & Down & 0.4217382 & 0.8823614 \\
\hline miR-106a-3p & -0.0896953 & Down & 0.0139619 & 0.46600109 \\
\hline miR-4639-3p & -0.0890044 & Down & 0.02945662 & 0.59616058 \\
\hline miR-548L & -0.0889439 & Down & 0.01327019 & 0.46067162 \\
\hline miR-6856-3p & -0.088886 & Down & 0.01234784 & 0.44695703 \\
\hline
\end{tabular}

(Continued) 
Table 9 (Continued).

\begin{tabular}{|c|c|c|c|c|}
\hline miRNAs & Log2FoldChange & Regulation & P-value & P-adj \\
\hline miR-3192-3p & -0.0887498 & Down & 0.01718806 & 0.53220869 \\
\hline miR-550a-3p & $-0.08804 I$ & Down & 0.36796855 & 0.86195734 \\
\hline miR-I25b-I-3p & -0.0877112 & Down & 0.24414246 & 0.80715125 \\
\hline miR-4668-3p & -0.0875502 & Down & 0.02816443 & 0.59258245 \\
\hline miR-6082 & -0.0865329 & Down & 0.15071067 & 0.75393664 \\
\hline miR-574-3p & -0.0854029 & Down & 0.47265855 & $0.8939601 \mathrm{I}$ \\
\hline miR-3606-5p & -0.0844866 & Down & 0.02275562 & 0.53734625 \\
\hline miR-148a-5p & -0.0830521 & Down & 0.01814262 & 0.53734625 \\
\hline miR-664a-3p & -0.0828903 & Down & 0.55610772 & 0.9268462 \\
\hline$m i R-6826-5 p$ & -0.0828478 & Down & 0.28421782 & 0.83193599 \\
\hline$m i R-54 I-5 p$ & -0.0823983 & Down & $0.0389 \mid 282$ & 0.59871978 \\
\hline miR-6884-3p & -0.0821635 & Down & 0.15347294 & 0.75393664 \\
\hline$m i R-3 \mid 24-5 p$ & -0.082068 & Down & 0.11585557 & 0.74226488 \\
\hline$m i R-3616-3 p$ & -0.0817705 & Down & 0.0990518 & 0.73085631 \\
\hline miR-6I8 & $-0.08 \mid 4829$ & Down & 0.01137484 & 0.43937422 \\
\hline miR-629-5p & $-0.08 \mid 454$ & Down & 0.32855493 & 0.85489649 \\
\hline miR-6784-5p & $-0.08|4| 67$ & Down & 0.03529383 & $0.5987 / 978$ \\
\hline$m i R-425-5 p$ & -0.0813279 & Down & 0.23355464 & 0.80019585 \\
\hline miR-426I & -0.0812625 & Down & 0.16684807 & $0.769 \mid 5081$ \\
\hline$m i R-4482-3 p$ & -0.0811715 & Down & 0.04757793 & $0.614 \mid 2501$ \\
\hline miR-|29I & -0.0810705 & Down & 0.14627622 & 0.75376261 \\
\hline miR-3I58-3p & -0.0800994 & Down & 0.077668898 & 0.68910078 \\
\hline miR-590-3p & -0.0792172 & Down & 0.03519026 & 0.59871978 \\
\hline miR-26a-I-3p & $-0.079|54|$ & Down & 0.17249915 & 0.77062416 \\
\hline$m i R-68 \mid 3-5 p$ & -0.0783623 & Down & 0.02180293 & 0.53734625 \\
\hline let-7c-3p & -0.0778908 & Down & 0.11832515 & 0.74226488 \\
\hline miR-150-5p & -0.07768 & Down & 0.4987205 I & 0.91032268 \\
\hline miR-5I90 & -0.0774762 & Down & 0.15597493 & 0.75633126 \\
\hline miR-20b-5p & -0.0772418 & Down & 0.1460428 & 0.75376261 \\
\hline miR-4270 & -0.0770622 & Down & 0.38633037 & $0.8696 \mid 208$ \\
\hline miR-3I-3p & -0.076925 & Down & 0.2362799 & 0.80380192 \\
\hline miR-5088-3p & -0.0768011 & Down & 0.09382537 & 0.72555728 \\
\hline miR-65I Ib-3p & -0.076708 & Down & 0.02162468 & 0.53734625 \\
\hline miR-196a-5p & -0.0765723 & Down & 0.27611079 & 0.83108541 \\
\hline miR-I249-3p & -0.0757546 & Down & 0.2545288 & 0.81031406 \\
\hline miR-9-5p & -0.0751537 & Down & 0.12923434 & 0.74892227 \\
\hline$m i R-6516-5 p$ & -0.0745078 & Down & 0.06014748 & 0.64832335 \\
\hline$m i R-4687-5 p$ & -0.0740003 & Down & 0.30255863 & 0.83872374 \\
\hline miR-673I-5p & -0.0739289 & Down & 0.03193883 & $0.5987 \mid 978$ \\
\hline miR-|4|-5p & -0.0733955 & Down & $0.0795745 \mathrm{I}$ & 0.68910078 \\
\hline miR-4780 & -0.073278 & Down & 0.1286269 & 0.74892227 \\
\hline miR-374b-3p & -0.0731784 & Down & 0.06692731 & 0.65890175 \\
\hline miR-369-5p & -0.0731604 & Down & 0.57647798 & 0.93390911 \\
\hline let-7d-3p & -0.0728777 & Down & 0.17184823 & 0.77062416 \\
\hline miR-66I & -0.0728391 & Down & 0.03624906 & $0.5987 / 978$ \\
\hline miR-649 & -0.0727858 & Down & 0.04419742 & $0.614|250|$ \\
\hline miR-4298 & -0.0724915 & Down & 0.47222756 & 0.89396011 \\
\hline miR-4683 & -0.0724638 & Down & 0.0546774 & 0.64056718 \\
\hline miR-5692c & -0.0723209 & Down & 0.08824084 & 0.71090581 \\
\hline
\end{tabular}

(Continued) 
Table 9 (Continued).

\begin{tabular}{|c|c|c|c|c|}
\hline miRNAs & Log2FoldChange & Regulation & P-value & P-adj \\
\hline miR-4688 & -0.0717924 & Down & 0.1290666 & 0.74892227 \\
\hline miR-874-5p & -0.0713546 & Down & 0.03569465 & 0.59871978 \\
\hline miR-654-3p & $-0.07 \mid 2607$ & Down & 0.77258369 & 0.98440262 \\
\hline miR-6772-5p & -0.0708382 & Down & 0.14810668 & $0.7537626 \mathrm{I}$ \\
\hline miR-36I-5p & -0.0707358 & Down & 0.46871632 & $0.8939601 \mathrm{I}$ \\
\hline miR-526b-3p & -0.069705 I & Down & 0.14424637 & $0.7537626 \mathrm{I}$ \\
\hline miR-5I5-5p & -0.069631 & Down & $0.03|5665|$ & 0.59871978 \\
\hline miR-7I56-5p & -0.0690574 & Down & 0.1545765 & 0.75393664 \\
\hline miR-6886-3p & -0.0685914 & Down & 0.12854264 & 0.74892227 \\
\hline miR-16-2-3p & -0.0684503 & Down & 0.21995732 & 0.78841048 \\
\hline miR-473I-3p & -0.0681986 & Down & 0.30047948 & 0.83872374 \\
\hline $\operatorname{miR}-548 a x$ & -0.0680894 & Down & 0.14111828 & $0.7537626 \mathrm{I}$ \\
\hline miR-296-3p & -0.0678209 & Down & 0.10482914 & 0.74226488 \\
\hline miR-664a-5p & -0.0677982 & Down & $0.4525 \mid 254$ & 0.89093879 \\
\hline miR-655-5p & -0.06773 II & Down & 0.10801005 & 0.74226488 \\
\hline miR-4680-3p & -0.0675655 & Down & 0.21604469 & 0.7853205 \\
\hline miR-6749-5p & -0.0673259 & Down & 0.33082272 & 0.85489649 \\
\hline miR-337-3p & -0.06711 & Down & 0.55060733 & 0.92349191 \\
\hline miR-504-3p & -0.0669662 & Down & 0.14725315 & $0.7537626 \mathrm{I}$ \\
\hline miR-758-3p & -0.0665848 & Down & 0.62687523 & $0.949834 \mid$ \\
\hline miR-937-3p & $-0.066544 \mid$ & Down & 0.05297921 & 0.63842356 \\
\hline miR-548av-3p & -0.066494 & Down & 0.08687757 & 0.70907074 \\
\hline miR-5572 & $-0.066|79|$ & Down & 0.05630095 & 0.64832335 \\
\hline miR-140-5p & -0.066004 I & Down & 0.45375538 & 0.89093879 \\
\hline miR-I227-5p & -0.0658486 & Down & $0.0369 \mid 231$ & 0.59871978 \\
\hline miR-4776-5p & $-0.065786 \mid$ & Down & 0.03971689 & 0.59871978 \\
\hline miR-548ab & -0.0655982 & Down & 0.10855319 & 0.74226488 \\
\hline miR-15b-3p & -0.0655518 & Down & 0.07743768 & 0.68910078 \\
\hline miR-6796-3p & -0.0655189 & Down & 0.12426931 & 0.74454632 \\
\hline miR-I233-5p & -0.0654842 & Down & 0.15416195 & 0.75393664 \\
\hline miR-922 & -0.0653003 & Down & 0.08895039 & $0.7|4| 1 \mid 286$ \\
\hline miR-5189-3p & -0.0649979 & Down & $0.1555889 \mid$ & 0.75588563 \\
\hline miR-4267 & -0.0648969 & Down & 0.03895436 & 0.59871978 \\
\hline miR-7I62-5p & -0.0647263 & Down & $0 .|8856| 2 \mid$ & $0.7799749 \mid$ \\
\hline$m i R-33 a-3 p$ & -0.0642384 & Down & 0.11657237 & 0.74226488 \\
\hline miR-942-5p & -0.0637306 & Down & 0.06524443 & 0.65598378 \\
\hline miR-134-5p & -0.0637176 & Down & 0.44938568 & 0.88964976 \\
\hline miR-539-5p & -0.0636818 & Down & 0.46151992 & 0.89396011 \\
\hline miR-28-3p & -0.063576 & Down & 0.19253465 & $0.7799749 \mid$ \\
\hline miR-608I & -0.0634098 & Down & 0.2394534 & 0.80380192 \\
\hline miR-7854-3p & -0.0633369 & Down & 0.08215352 & 0.69839839 \\
\hline miR-624-5p & -0.0632708 & Down & 0.26163925 & 0.81989814 \\
\hline miR-2II5-3p & -0.0630218 & Down & 0.07815829 & 0.68910078 \\
\hline miR-4652-3p & -0.063004 & Down & 0.24882332 & $0.807|5| 25$ \\
\hline$m i R-489-5 p$ & -0.0629817 & Down & 0.05217839 & 0.63253996 \\
\hline $\operatorname{miR}-212-5 p$ & -0.062839 & Down & 0.19944415 & 0.7799749 | \\
\hline miR-1273e & -0.0623133 & Down & 0.10094184 & 0.73085631 \\
\hline miR-134-3p & -0.0622349 & Down & $0 .|772474|$ & 0.77062416 \\
\hline miR-6875-3p & -0.0617556 & Down & 0.1375524 & $0.7537626 \mathrm{I}$ \\
\hline
\end{tabular}

(Continued) 
Table 9 (Continued).

\begin{tabular}{|c|c|c|c|c|}
\hline miRNAs & Log2FoldChange & Regulation & P-value & P-adj \\
\hline miR-219a-5p & -0.0616904 & Down & 0.2533948 & 0.80998089 \\
\hline miR-493-3p & -0.0611597 & Down & 0.09806579 & 0.73085631 \\
\hline$m i R-688 I-5 p$ & -0.0609269 & Down & 0.09484994 & 0.72765477 \\
\hline miR-125a-3p & -0.0608378 & Down & 0.21286419 & 0.78263372 \\
\hline miR-4439 & $-0.060587 \mid$ & Down & 0.1233437 & 0.74454632 \\
\hline miR-4730 & -0.0605398 & Down & 0.06998566 & 0.67187824 \\
\hline miR-383-3p & -0.0604946 & Down & 0.09662856 & 0.73085631 \\
\hline miR-548ay-3p & -0.060446 & Down & 0.03093749 & $0.5978|47|$ \\
\hline miR-3।80-3p & -0.0601756 & Down & 0.07181798 & $0.68344 \mid 28$ \\
\hline miR-10b-3p & $-0.0598 \mid 46$ & Down & 0.11849442 & 0.74226488 \\
\hline miR-539-3p & -0.05978 & Down & 0.12258423 & 0.74454632 \\
\hline let-7d-5p & -0.0595976 & Down & 0.2632232 & 0.82197281 \\
\hline miR-18Ib-2-3p & -0.0595461 & Down & 0.05810103 & 0.64832335 \\
\hline miR-5683 & -0.0595096 & Down & 0.29822618 & 0.83872374 \\
\hline$m i R-5 \mid 7 c-3 p$ & $-0.059 \mid 456$ & Down & 0.111974 & 0.74226488 \\
\hline miR-II85-I-3p & -0.0591174 & Down & $0.73750 \mid 48$ & 0.97354829 \\
\hline$m i R-432-3 p$ & -0.0589101 & Down & 0.15090885 & 0.75393664 \\
\hline miR-192-3p & -0.0588899 & Down & 0.20081511 & 0.77997491 \\
\hline miR-30lb-3p & -0.0588816 & Down & 0.41071015 & 0.8793356 \\
\hline miR-550a-3-5p & -0.0588116 & Down & 0.30975262 & 0.84687685 \\
\hline miR-5090 & -0.0587267 & Down & 0.09431345 & 0.72570526 \\
\hline miR-4700-3p & $-0.0586 \mid 54$ & Down & 0.16039599 & $0.76082 \mid 42$ \\
\hline$m i R-26 b-3 p$ & -0.058326 & Down & 0.13777214 & 0.75376261 \\
\hline miR-8087 & -0.0582875 & Down & 0.11944341 & 0.74226488 \\
\hline miR-138-2-3p & -0.0580683 & Down & 0.04430432 & $0.614|250|$ \\
\hline miR-3I63 & -0.05801 & Down & $0.065343 \mid 3$ & 0.65598378 \\
\hline miR-877-5p & -0.0578883 & Down & 0.02966172 & 0.59616058 \\
\hline miR-3|87-3p & -0.0574442 & Down & 0.06122058 & 0.6501524 \\
\hline miR-6752-5p & $-0.057 \mid 494$ & Down & 0.23470494 & 0.80105138 \\
\hline$m i R-684 I-3 p$ & -0.0569437 & Down & 0.25203953 & 0.80715125 \\
\hline miR-I 322 & -0.056926 & Down & 0.2324719 & 0.79873366 \\
\hline$m i R-668-3 p$ & -0.0565419 & Down & 0.29465932 & 0.83872374 \\
\hline miR-4668-5p & $-0.056380 \mathrm{I}$ & Down & 0.07717657 & 0.68910078 \\
\hline miR-36I5 & -0.0563464 & Down & 0.17425676 & 0.77062416 \\
\hline miR-409-3p & -0.0559845 & Down & $0.87|64| 83$ & $0.998987 \mid 2$ \\
\hline miR-154-5p & -0.0555697 & Down & 0.75862106 & 0.98140055 \\
\hline miR-5699-3p & -0.0554852 & Down & 0.17600494 & 0.77062416 \\
\hline miR-632 & -0.0552967 & Down & 0.07228476 & $0.68344 \mid 28$ \\
\hline miR-7I57-5p & -0.0549047 & Down & 0.07072583 & 0.67570774 \\
\hline miR-4464 & -0.0548509 & Down & 0.22364029 & 0.79102241 \\
\hline$m i R-4446-3 p$ & -0.0545982 & Down & 0.41195333 & 0.8793356 \\
\hline miR-8083 & -0.0545119 & Down & $0.2004 I 269$ & 0.77997491 \\
\hline miR-30e-5p & -0.0545001 & Down & 0.60176327 & 0.93953261 \\
\hline miR-595 & $-0.054295 I$ & Down & 0.07233309 & $0.68344 \mid 28$ \\
\hline miR-6505-5p & -0.0539884 & Down & 0.09749892 & 0.73085631 \\
\hline$m i R-4686$ & -0.0539254 & Down & 0.04492707 & $0.614|250|$ \\
\hline miR-449b-5p & -0.0538689 & Down & $0.0993349 \mid$ & 0.73085631 \\
\hline$m i R-4693-5 p$ & -0.0538069 & Down & 0.09863654 & 0.73085631 \\
\hline miR-4297 & -0.0537088 & Down & $0.148 \mid 4504$ & 0.75376261 \\
\hline
\end{tabular}

(Continued) 
Table 9 (Continued).

\begin{tabular}{|c|c|c|c|c|}
\hline miRNAs & Log2FoldChange & Regulation & P-value & P-adj \\
\hline miR-1237-3p & -0.053556 & Down & 0.0995295 & 0.73085631 \\
\hline miR-6739-3p & -0.053498 I & Down & 0.21199749 & 0.78263372 \\
\hline miR-5I3a-3p & -0.0534758 & Down & 0.27013449 & 0.83107769 \\
\hline miR-369I-3p & -0.0526913 & Down & 0.08393675 & 0.70009532 \\
\hline miR-6764-5p & -0.0526382 & Down & 0.06180898 & $0.65 I 02084$ \\
\hline miR-I245b-5p & -0.052259 & Down & 0.18642439 & 0.77777707 \\
\hline miR-5I94 & -0.0519302 & Down & 0.31143873 & $0.8475 \mid 25$ \\
\hline miR-4520-2-3p & -0.0519039 & Down & 0.0572582 & 0.64832335 \\
\hline miR-I52-5p & -0.0518119 & Down & 0.27837305 & $0.83|0854|$ \\
\hline miR-4446-5p & -0.0517576 & Down & 0.11542673 & 0.74226488 \\
\hline$m i R-449 c-5 p$ & -0.0517263 & Down & 0.17444718 & 0.77062416 \\
\hline miR-4708-3p & $-0.0517 \mid 26$ & Down & $0.087 \mid 8535$ & 0.70907074 \\
\hline miR-4659b-3p & -0.051703 & Down & 0.18474022 & 0.77578819 \\
\hline miR-7706 & -0.051404 & Down & 0.03554956 & $0.5987 / 978$ \\
\hline miR-6837-3p & -0.0513624 & Down & 0.07988723 & 0.68910078 \\
\hline miR-6757-5p & -0.051295 & Down & 0.37187056 & $0.8648935 I$ \\
\hline miR-4798-5p & -0.0512733 & Down & 0.16679352 & $0.769|508|$ \\
\hline miR-6832-3p & $-0.05 \mid 2067$ & Down & 0.20749798 & 0.78263372 \\
\hline miR-30c-5p & $-0.05 I 185$ & Down & 0.3283045 & 0.85489649 \\
\hline miR-I266-3p & -0.051152 & Down & 0.15251966 & 0.75393664 \\
\hline miR-I 262 & -0.0506055 & Down & 0.1481356 & $0.7537626 \mathrm{I}$ \\
\hline miR-885-5p & -0.0505099 & Down & 0.23316458 & 0.80004402 \\
\hline miR-3529-3p & -0.0503778 & Down & 0.29076307 & 0.83872374 \\
\hline miR-2052 & -0.0503661 & Down & $0.2437297 \mid$ & $0.807 / 5 I 25$ \\
\hline$m i R-4743-5 p$ & -0.0503648 & Down & 0.33589778 & 0.85489649 \\
\hline miR-125b-2-3p & -0.0502628 & Down & 0.1992263 & 0.7799749 I \\
\hline miR-4275 & -0.0501719 & Down & 0.23383155 & 0.80019585 \\
\hline miR-3I66 & $-0.050 I 55$ & Down & 0.11485278 & 0.74226488 \\
\hline miR-4492 & -0.0499947 & Down & 0.23805179 & 0.80380192 \\
\hline miR-6864-5p & -0.0499078 & Down & 0.21015153 & 0.78263372 \\
\hline miR-384 & -0.049905 & Down & 0.19524287 & 0.7799749 | \\
\hline miR-6885-3p & -0.0495386 & Down & 0.2725345 & 0.83107769 \\
\hline miR-4665-5p & -0.0494764 & Down & 0.1995748 & 0.7799749 I \\
\hline miR-6865-5p & -0.0494428 & Down & 0.20017313 & 0.7799749 I \\
\hline miR-3977 & -0.0491608 & Down & 0.217245 & 0.7863657 I \\
\hline miR-6873-3p & -0.0490828 & Down & 0.4649756 & $0.893960 \mathrm{II}$ \\
\hline miR-3|36-5p & -0.0490032 & Down & 0.1782882 & 0.77062416 \\
\hline miR-550b-3p & -0.0489921 & Down & 0.21365444 & $0.7844 I 702$ \\
\hline miR-II 97 & -0.0487257 & Down & 0.25155855 & $0.807|5| 25$ \\
\hline miR-4487 & -0.048424 & Down & 0.16645497 & $0.769|508|$ \\
\hline miR-769-3p & -0.0484013 & Down & $0.3 \mid 433383$ & 0.84917098 \\
\hline miR-45I 9 & -0.0483057 & Down & 0.07649724 & 0.68910078 \\
\hline miR-647 & -0.0482847 & Down & 0.35163792 & 0.8588192 \\
\hline miR-6728-5p & -0.0481616 & Down & 0.39964052 & 0.87364718 \\
\hline$m i R-2 I I 4-5 p$ & $-0.04806 I I$ & Down & $0.104|378|$ & 0.74226488 \\
\hline miR-488-3p & $-0.0479|2|$ & Down & 0.22846615 & $0.79|0224|$ \\
\hline$m i R-4633-5 p$ & -0.0477808 & Down & 0.14610901 & $0.7537626 \mathrm{I}$ \\
\hline miR-6794-3p & -0.0476669 & Down & 0.28649085 & $0.8328976 \mathrm{I}$ \\
\hline miR-4522 & -0.0476432 & Down & 0.23913101 & 0.80380192 \\
\hline
\end{tabular}

(Continued) 
Table 9 (Continued).

\begin{tabular}{|c|c|c|c|c|}
\hline miRNAs & Log2FoldChange & Regulation & P-value & P-adj \\
\hline miR-95-5p & -0.0471746 & Down & 0.28640069 & 0.83289761 \\
\hline miR-65I0-5p & $-0.047 \mid 527$ & Down & 0.22252947 & 0.79102241 \\
\hline miR-6738-5p & -0.0466691 & Down & 0.292255 & 0.83872374 \\
\hline miR-887-5p & -0.0465034 & Down & 0.20770552 & 0.78263372 \\
\hline miR-52I & -0.046491 & Down & 0.14795142 & 0.75376261 \\
\hline miR-316I & -0.0464806 & Down & 0.22632853 & 0.79102241 \\
\hline miR-4754 & -0.0463985 & Down & 0.19022343 & 0.77997491 \\
\hline miR-3680-5p & -0.0463564 & Down & 0.08455247 & 0.70096725 \\
\hline miR-503-3p & -0.0463332 & Down & 0.18006004 & 0.77062416 \\
\hline miR-3074-5p & -0.0463157 & Down & 0.05369175 & 0.63883244 \\
\hline miR-I 254 & -0.046131 & Down & 0.09134723 & 0.72012996 \\
\hline miR-578 & $-0.046 \mid I 82$ & Down & $0.2368 \mid 458$ & 0.80380192 \\
\hline miR-3667-3p & -0.0459469 & Down & 0.20349294 & 0.77997491 \\
\hline miR-1273h-5p & -0.0459311 & Down & 0.17028256 & 0.77062416 \\
\hline$m i R-1224-3 p$ & -0.0457998 & Down & 0.14643085 & 0.75376261 \\
\hline miR-6793-5p & -0.0457419 & Down & $0.25446 \mid 21$ & 0.81031406 \\
\hline miR-6770-5p & -0.045575 & Down & 0.11368816 & 0.74226488 \\
\hline$m i R-423-5 p$ & -0.0455524 & Down & 0.63293665 & 0.95096807 \\
\hline miR-99a-3p & -0.0455344 & Down & 0.17224632 & 0.77062416 \\
\hline miR-205-3p & $-0.04531 \mid 4$ & Down & 0.10376792 & 0.74226488 \\
\hline miR-58I & -0.0451704 & Down & 0.20455373 & 0.7799749 \\
\hline miR-1269a & -0.0451647 & Down & 0.16385234 & 0.76892691 \\
\hline miR-5690 & -0.0451396 & Down & 0.45378945 & 0.89093879 \\
\hline miR-6830-5p & -0.0450438 & Down & 0.16855939 & 0.77062416 \\
\hline miR-6758-3p & -0.044947 & Down & 0.30113939 & 0.83872374 \\
\hline miR-3I33 & -0.044896 & Down & 0.24881996 & $0.807|5| 25$ \\
\hline$m i R-1468-5 p$ & -0.0447274 & Down & 0.35320208 & 0.8588192 \\
\hline miR-675-5p & -0.0446309 & Down & $0.0756789 \mid$ & 0.68726073 \\
\hline miR-6808-3p & -0.0446026 & Down & 0.17878595 & 0.77062416 \\
\hline miR-558 & -0.0445564 & Down & 0.20364686 & 0.7799749 \\
\hline miR-4282 & -0.0445282 & Down & 0.3024577 I & 0.83872374 \\
\hline$m i R-584-3 p$ & $-0.044494 I$ & Down & 0.1360289 & 0.75376261 \\
\hline miR-577 & -0.0442789 & Down & 0.22696156 & 0.79102241 \\
\hline miR-802 & -0.044274 & Down & 0.32802998 & 0.85489649 \\
\hline miR-I303 & -0.0440308 & Down & 0.06970506 & 0.67187824 \\
\hline miR-89lb & -0.0439852 & Down & 0.14633769 & 0.75376261 \\
\hline miR-4483 & $-0.04388 \mid 8$ & Down & $0.17627 \mid 44$ & 0.77062416 \\
\hline miR-302b-5p & -0.0438292 & Down & 0.19508308 & 0.77997491 \\
\hline miR-I343-5p & -0.0436694 & Down & 0.20551786 & 0.78047751 \\
\hline miR-4742-5p & -0.0432328 & Down & $0.287 \mid 3222$ & 0.83381898 \\
\hline miR-6785-3p & -0.043203 & Down & 0.53395073 & 0.9213181 \\
\hline$m i R-5000-5 p$ & -0.0430893 & Down & 0.16801664 & 0.77062416 \\
\hline miR-222-5p & -0.0429509 & Down & $0.1878825 \mid$ & 0.7799749I \\
\hline miR-II78-5p & -0.0428242 & Down & 0.29950566 & 0.83872374 \\
\hline miR-548k & -0.042777 & Down & 0.11181738 & 0.74226488 \\
\hline$m i R-486-3 p$ & -0.042765 & Down & $0.35734 \mid 47$ & 0.8588192 \\
\hline miR-516a-5p & -0.0425529 & Down & 0.42420656 & 0.8823614 \\
\hline miR-487b-5p & -0.0424956 & Down & $0.2752760 \mathrm{I}$ & $0.8310854 \mid$ \\
\hline miR-4470 & -0.0424497 & Down & 0.18738773 & 0.77997491 \\
\hline
\end{tabular}

(Continued) 
Table 9 (Continued).

\begin{tabular}{|c|c|c|c|c|}
\hline miRNAs & Log2FoldChange & Regulation & P-value & P-adj \\
\hline miR-3197 & -0.0424123 & Down & 0.40575495 & 0.87475982 \\
\hline miR-223-5p & -0.0423677 & Down & 0.77529043 & 0.985258 \\
\hline miR-593-3p & -0.0423211 & Down & 0.18015658 & 0.77062416 \\
\hline$m i R-65 I-5 p$ & $-0.0422 \mid 48$ & Down & 0.33409291 & 0.85489649 \\
\hline miR-6854-3p & -0.0421954 & Down & $0.3735 \mid 408$ & 0.8649907| \\
\hline miR-44I9b & -0.04196 & Down & 0.22498221 & $0.79|0224|$ \\
\hline miR-4728-3p & -0.0419086 & Down & 0.11897989 & 0.74226488 \\
\hline miR-4268 & -0.0419035 & Down & 0.13293728 & 0.74892227 \\
\hline miR-518c-3p & $-0.04|8| 5 \mid$ & Down & 0.29336201 & 0.83872374 \\
\hline$m i R-47 \mid 2-3 p$ & -0.0416429 & Down & $0.2256407 \mid$ & $0.79|0224|$ \\
\hline miR-47II-5p & -0.0416366 & Down & 0.19518099 & $0.7799749 \mid$ \\
\hline miR-3692-3p & -0.0416267 & Down & 0.1156964 & 0.74226488 \\
\hline miR-4280 & -0.0416189 & Down & 0.25672311 & 0.81105473 \\
\hline $\operatorname{miR}-4314$ & $-0.04|4| 78$ & Down & 0.15047172 & 0.75393664 \\
\hline miR-6732-5p & $-0.04|392|$ & Down & 0.33293924 & 0.85489649 \\
\hline miR-7I06-3p & -0.0413412 & Down & 0.25137777 & $0.807|5| 25$ \\
\hline miR-559I-3p & -0.04123 & Down & 0.29994998 & 0.83872374 \\
\hline miR-7705 & -0.041217 & Down & 0.0634476 & 0.65539395 \\
\hline miR-6855-3p & -0.0410619 & Down & 0.45728436 & $0.8924444 \mid$ \\
\hline miR-568 & -0.0408745 & Down & 0.33324655 & 0.85489649 \\
\hline miR-1343-3p & -0.0408367 & Down & 0.24415703 & 0.80715125 \\
\hline miR-478I-5p & -0.0407069 & Down & 0.13214069 & 0.74892227 \\
\hline$m i R-49 I-3 p$ & -0.0406686 & Down & 0.22868858 & $0.79|0224|$ \\
\hline miR-4707-5p & -0.0405516 & Down & 0.22322314 & $0.79|0224|$ \\
\hline miR-3 I29-5p & -0.0401239 & Down & 0.25161482 & $0.807|5| 25$ \\
\hline miR-6086 & -0.0401014 & Down & 0.4948927 & 0.90848159 \\
\hline miR-203a-3p & -0.0400958 & Down & 0.34293226 & 0.8578521 \\
\hline NC2_00I2273I & -0.0399138 & Down & 0.19213775 & $0.7799749 \mid$ \\
\hline miR-3065-3p & -0.0399098 & Down & $0.224858 I I$ & $0.79|0224|$ \\
\hline miR-5585-5p & -0.0399008 & Down & 0.3792633 & 0.8649907| \\
\hline$m i R-576-5 p$ & -0.0397585 & Down & 0.35921288 & 0.8588192 \\
\hline miR-374c-3p & -0.0395254 & Down & 0.35134596 & 0.8588192 \\
\hline miR-5009-3p & -0.0391632 & Down & 0.31867916 & $0.8495907 \mid$ \\
\hline miR-4303 & -0.0390723 & Down & 0.0998174 & 0.73085631 \\
\hline$m i R-4662 a-5 p$ & -0.0390549 & Down & 0.48355794 & 0.90449982 \\
\hline miR-6868-3p & -0.0390428 & Down & 0.11010451 & 0.74226488 \\
\hline miR-4525 & -0.0389599 & Down & 0.21635149 & 0.7853205 \\
\hline miR-I269b & -0.038945 I & Down & 0.31640306 & 0.84917098 \\
\hline miR-329-3p & -0.038917 & Down & 0.71268513 & 0.96505269 \\
\hline miR-6500-3p & -0.0388368 & Down & 0.21551632 & 0.7853205 \\
\hline miR-3I77-3p & -0.0387482 & Down & 0.23821507 & 0.80380192 \\
\hline miR-142-3p & -0.0387095 & Down & 0.80992531 & 0.99064883 \\
\hline$m i R-2 \mid 2-3 p$ & -0.0386542 & Down & 0.4975312 & 0.91032268 \\
\hline miR-623 & -0.0385079 & Down & 0.19978928 & | 0.7799749 \\
\hline$m i R-518 e-3 p$ & -0.038422 & Down & 0.3563435 & 0.8588192 \\
\hline miR-3658 & -0.0384105 & Down & 0.27866195 & $0.83|0854|$ \\
\hline miR-6744-3p & -0.0384055 & Down & $0.2007 \mid 422$ & $0.7799749 \mid$ \\
\hline miR-6755-3p & -0.0382967 & Down & 0.19995738 & $0.7799749 \mid$ \\
\hline miR-4423-3p & -0.03821 & Down & 0.3154744 & 0.84917098 \\
\hline
\end{tabular}

(Continued) 
Table 9 (Continued).

\begin{tabular}{|c|c|c|c|c|}
\hline miRNAs & Log2FoldChange & Regulation & P-value & P-adj \\
\hline miR-4445-3p & $-0.038 \mid 457$ & Down & 0.38961402 & $0.8728 \mid 803$ \\
\hline miR-5582-5p & -0.0380637 & Down & 0.12812294 & 0.74892227 \\
\hline miR-39l7 & -0.037903 & Down & 0.26173436 & 0.81989814 \\
\hline miR-873-5p & -0.0377577 & Down & 0.41493701 & 0.8804535 \\
\hline miR-480I & -0.0377502 & Down & $0.346302 \mid 4$ & 0.8588192 \\
\hline$m i R-548 e-3 p$ & -0.0376306 & Down & $0.3382061 \mathrm{I}$ & 0.85489649 \\
\hline miR-I285-3p & -0.0374564 & Down & 0.19647604 & 0.77997491 \\
\hline miR-4790-3p & -0.03735 & Down & 0.20157842 & 0.77997491 \\
\hline$m i R-683 I-3 p$ & $-0.037278 I$ & Down & 0.58185088 & 0.93436563 \\
\hline miR-508-3p & -0.037225 & Down & 0.23101382 & 0.79502561 \\
\hline miR-204-3p & -0.0371964 & Down & 0.45469283 & 0.891297 \\
\hline miR-8079 & $-0.037 \mid 74$ & Down & 0.21082788 & 0.78263372 \\
\hline miR-6726-3p & -0.0370802 & Down & 0.27602556 & 0.83108541 \\
\hline miR-3938 & -0.0369838 & Down & 0.15952768 & 0.76064219 \\
\hline$m i R-3152-3 p$ & -0.0368768 & Down & 0.21985777 & 0.78841048 \\
\hline miR-54I-3p & -0.0368742 & Down & 0.25219551 & 0.80715125 \\
\hline miR-2I-5p & -0.0368457 & Down & $0.89594 \mid 26$ & $0.998987 \mid 2$ \\
\hline miR-4257 & -0.0367867 & Down & 0.53676574 & 0.9213181 \\
\hline miR-3I73-5p & -0.036755 & Down & 0.43342459 & 0.88898739 \\
\hline miR-6866-3p & -0.0365902 & Down & 0.260655 & 0.8189283 \\
\hline$m i R-130 I-5 p$ & -0.0365849 & Down & 0.39157163 & $0.8728 \mid 803$ \\
\hline miR-608 & -0.0364538 & Down & 0.20435884 & 0.77997491 \\
\hline miR-3923 & -0.0363088 & Down & 0.43136219 & 0.88759074 \\
\hline miR-5I93 & -0.0362094 & Down & $0.334|270|$ & 0.85489649 \\
\hline miR-509-5p & -0.0361572 & Down & 0.31939227 & 0.84972893 \\
\hline miR-4509 & -0.0360699 & Down & 0.22833098 & 0.79102241 \\
\hline miR-I 289 & -0.0360075 & Down & 0.30327032 & 0.83872374 \\
\hline$m i R-6503-5 p$ & -0.0359935 & Down & 0.50191361 & 0.91147569 \\
\hline miR-106b-5p & $-0.035946 \mid$ & Down & 0.61507233 & 0.94689808 \\
\hline let-7a-3p & -0.0357979 & Down & $0.5629640 \mathrm{I}$ & 0.92970327 \\
\hline$m i R-5 \mid 8 f-5 p$ & -0.0357722 & Down & 0.33145655 & 0.85489649 \\
\hline miR-47I8 & -0.0357336 & Down & 0.43521457 & $0.8894 I 554$ \\
\hline miR-2682-5p & -0.0357322 & Down & 0.15095123 & 0.75393664 \\
\hline miR-766-5p & $-0.0357 \mid 83$ & Down & 0.31558575 & 0.84917098 \\
\hline NegativeControl & -0.035584 & Down & 0.13903884 & 0.75376261 \\
\hline miR-5587-3p & -0.0355696 & Down & 0.1775629 & 0.77062416 \\
\hline$m i R-27 b-5 p$ & -0.0355676 & Down & 0.39363988 & 0.87310741 \\
\hline miR-500b-3p & -0.0355306 & Down & 0.36316867 & 0.8588192 \\
\hline miR-4735-5p & $-0.0355 \mid 25$ & Down & 0.4156067 & 0.8804535 \\
\hline miR-67।6-5p & -0.0354956 & Down & 0.19348116 & 0.77997491 \\
\hline miR-676-5p & -0.0354686 & Down & 0.44701629 & 0.88952595 \\
\hline miR-7III-3p & -0.0353822 & Down & 0.52941856 & 0.92119547 \\
\hline$m i R-422 a$ & -0.0352602 & Down & 0.43842522 & 0.88952595 \\
\hline miR-4722-3p & -0.0349901 & Down & 0.36975335 & 0.86231045 \\
\hline miR-7I06-5p & -0.0349495 & Down & 0.48820261 & 0.90656383 \\
\hline miR-299-5p & -0.0348796 & Down & 0.79516654 & 0.98759926 \\
\hline miR-6733-3p & -0.0348434 & Down & 0.43778503 & 0.88952595 \\
\hline miR-4322 & -0.0347927 & Down & $0.1987|57|$ & 0.77997491 \\
\hline miR-8062 & -0.0346104 & Down & 0.24081785 & 0.80586181 \\
\hline
\end{tabular}

(Continued) 
Table 9 (Continued).

\begin{tabular}{|c|c|c|c|c|}
\hline miRNAs & Log2FoldChange & Regulation & P-value & P-adj \\
\hline miR-6853-3p & -0.0346078 & Down & 0.25116815 & $0.807 / 5125$ \\
\hline miR-4757-5p & -0.0345925 & Down & 0.20637386 & 0.78227259 \\
\hline miR-3।44-3p & -0.0345136 & Down & 0.37885574 & 0.8649907 I \\
\hline miR-4799-3p & -0.0344395 & Down & 0.32812539 & 0.85489649 \\
\hline$m i R-4 \mid 2-3 p$ & -0.0343588 & Down & 0.31780759 & 0.84917098 \\
\hline$m i R-4703-5 p$ & -0.0341043 & Down & $0.4270085 I$ & 0.88287358 \\
\hline miR-4632-5p & -0.0340734 & Down & 0.2568866 & 0.81105473 \\
\hline miR-5689 & $-0.033697 \mid$ & Down & 0.33937179 & 0.85489649 \\
\hline$m i R-4708-5 p$ & -0.0334763 & Down & 0.19560352 & 0.7799749 I \\
\hline miR-674I-5p & -0.0334723 & Down & 0.38032665 & 0.8649907 I \\
\hline miR-4278 & -0.0334465 & Down & 0.24981153 & 0.80715125 \\
\hline miR-4636 & -0.033388 I & Down & 0.20375987 & 0.7799749 I \\
\hline miR-200a-5p & -0.0333265 & Down & 0.3457056 & 0.8588192 \\
\hline$m i R-5 \mid 3 c-3 p$ & -0.0332212 & Down & $0.44 I I 8445$ & 0.88952595 \\
\hline miR-5008-3p & -0.0331369 & Down & $0.29943 \mid 33$ & 0.83872374 \\
\hline$m i R-4666 a-5 p$ & -0.0331367 & Down & 0.41658812 & 0.8804535 \\
\hline miR-20a-5p & -0.033089 & Down & 0.47247238 & $0.893960 \mathrm{II}$ \\
\hline miR-3I75 & -0.033046 & Down & 0.37087896 & 0.86336859 \\
\hline miR-I324 & $-0.03298 \mid 8$ & Down & 0.31023739 & 0.84730085 \\
\hline miR-129-2-3p & -0.0328576 & Down & 0.62385115 & 0.9492584 I \\
\hline miR-I8b-3p & -0.032854 & Down & $0.5462 \mid 221$ & 0.92261663 \\
\hline miR-106b-3p & -0.0328101 & Down & $0.417 \mid 275$ & 0.88086907 \\
\hline miR-92I & -0.0327982 & Down & 0.33915794 & 0.85489649 \\
\hline miR-609 & -0.032727 & Down & 0.53862579 & 0.9213181 \\
\hline miR-1911-3p & -0.0326997 & Down & 0.46298065 & $0.893960 \mathrm{II}$ \\
\hline miR-637 & -0.0326982 & Down & $0.44 \mid 35535$ & 0.88952595 \\
\hline miR-430I & -0.0326704 & Down & 0.33862313 & 0.85489649 \\
\hline miR-19b-I-5p & -0.0326675 & Down & 0.43172229 & 0.88762102 \\
\hline miR-65I l b-5p & -0.0324825 & Down & 0.2042628 & 0.7799749 I \\
\hline miR-6I3 & -0.0322523 & Down & 0.48200927 & 0.9030564 \\
\hline miR-208a-5p & -0.0322369 & Down & 0.41564505 & 0.8804535 \\
\hline miR-6892-3p & -0.0322175 & Down & 0.65570287 & 0.95321673 \\
\hline miR-50I0-3p & -0.0321995 & Down & 0.49798778 & 0.91032268 \\
\hline miR-4737 & -0.0321658 & Down & 0.14010987 & $0.7537626 \mathrm{I}$ \\
\hline miR-548a-3p & -0.0321257 & Down & 0.53056959 & 0.9213181 \\
\hline miR-4259 & -0.0320631 & Down & $0.35247 \mid 29$ & 0.8588192 \\
\hline miR-4666b & -0.0319996 & Down & 0.31607306 & 0.84917098 \\
\hline miR-4747-3p & -0.0319254 & Down & 0.41641835 & 0.8804535 \\
\hline miR-6877-5p & -0.0319219 & Down & 0.37987848 & 0.8649907 I \\
\hline miR-548at-3p & $-0.03|8| 7 \mid$ & Down & 0.35020919 & 0.8588192 \\
\hline$m i R-678 I-5 p$ & -0.0317665 & Down & 0.33930583 & 0.85489649 \\
\hline miR-3686 & -0.0317569 & Down & 0.22396794 & $0.79|0224|$ \\
\hline miR-6842-5p & $-0.0317|0|$ & Down & 0.36890788 & $0.86 \mid 95734$ \\
\hline$m i R-6803-3 p$ & $-0.03 \mid 4802$ & Down & $0.40 \mid 46977$ & 0.87364718 \\
\hline miR-190a-5p & -0.0314616 & Down & 0.63180181 & 0.95096807 \\
\hline miR-450b-5p & $-0.03 \mid 4507$ & Down & 0.42557178 & 0.8823614 \\
\hline miR-24-3p & $-0.0314 \mid 97$ & Down & 0.75498569 & $0.98 \mid 40055$ \\
\hline miR-I8Id-3p & $-0.03|39|$ & Down & 0.27560752 & $0.83|0854|$ \\
\hline miR-477I & -0.0313002 & Down & 0.35849906 & 0.8588192 \\
\hline
\end{tabular}

(Continued) 
Table 9 (Continued).

\begin{tabular}{|c|c|c|c|c|}
\hline miRNAs & Log2FoldChange & Regulation & P-value & P-adj \\
\hline miR-25-5p & -0.0312979 & Down & 0.44199334 & 0.88952595 \\
\hline$m i R-2 \mid 8-5 p$ & -0.0312859 & Down & 0.33000051 & 0.85489649 \\
\hline miR-366I & -0.0312469 & Down & 0.15243214 & 0.75393664 \\
\hline miR-520b & -0.0312442 & Down & 0.44366687 & 0.88952595 \\
\hline miR-889-3p & $-0.03 \mid 2205$ & Down & $0.6408287 \mid$ & 0.95096807 \\
\hline miR-1912 & -0.0310354 & Down & 0.28102885 & 0.83108541 \\
\hline$m i R-65 \mid 2-5 p$ & -0.0309975 & Down & 0.48851369 & 0.90656383 \\
\hline miR-4709-5p & -0.0307474 & Down & 0.33313348 & 0.85489649 \\
\hline miR-520h & -0.0306452 & Down & 0.44723114 & 0.88952595 \\
\hline miR-6797-5p & -0.0306123 & Down & 0.35491367 & 0.8588192 \\
\hline$m i R-122-3 p$ & -0.0306052 & Down & 0.64051333 & 0.95096807 \\
\hline miR-4328 & -0.0306045 & Down & 0.30282113 & 0.83872374 \\
\hline miR-548ac & -0.0304654 & Down & 0.36242627 & 0.8588192 \\
\hline miR-6077 & -0.0304061 & Down & 0.33304636 & 0.85489649 \\
\hline$m i R-6853-5 p$ & -0.0303791 & Down & 0.3260448 & 0.85489649 \\
\hline miR-129-I-3p & -0.0303223 & Down & 0.60193873 & 0.93953261 \\
\hline miR-4540 & -0.030299 & Down & 0.20100239 & 0.77997491 \\
\hline$m i R-519 b-3 p$ & $-0.030 I 566$ & Down & 0.38005484 & $0.8649907 \mid$ \\
\hline miR-643 & -0.0301015 & Down & 0.44674239 & 0.88952595 \\
\hline miR-508-5p & -0.0299621 & Down & 0.39987343 & 0.87364718 \\
\hline miR-3। 83 & -0.0299339 & Down & 0.26463599 & 0.82438552 \\
\hline miR-6863 & -0.0298423 & Down & 0.2562445 I & 0.81102287 \\
\hline miR-5093 & -0.0297343 & Down & 0.3211177 & 0.85167439 \\
\hline miR-3II7-5p & -0.0296979 & Down & $0.3735 \mid 236$ & I 0.8649907 \\
\hline miR-1915-5p & -0.0295722 & Down & $0.386|459|$ & $0.8696 \mid 208$ \\
\hline$m i R-467 I-5 p$ & -0.0293784 & Down & 0.48029366 & 0.90296614 \\
\hline miR-7II $2-3 p$ & -0.0293344 & Down & $0.4069266 \mathrm{I}$ & 0.87475982 \\
\hline miR-6842-3p & -0.0292299 & Down & 0.37489059 & $0.8649907 \mid$ \\
\hline miR-7848-3p & -0.0291606 & Down & $0.47|7369|$ & 0.89396011 \\
\hline miR-3925-3p & $-0.029 \mid 496$ & Down & 0.44297755 & 0.88952595 \\
\hline miR-6075 & -0.0290637 & Down & 0.34793883 & 0.8588192 \\
\hline miR-6II & -0.0290556 & Down & 0.40338581 & 0.87475982 \\
\hline miR-3688-3p & -0.029053 & Down & 0.33899315 & 0.85489649 \\
\hline miR-7I5I-3p & -0.02883 II & Down & $0.2947 \mid 257$ & 0.83872374 \\
\hline$m i R-672 I-5 p$ & -0.0286954 & Down & $0.3367 \mid 486$ & 0.85489649 \\
\hline miR-499a-3p & -0.0286779 & Down & 0.3975788 & 0.87364718 \\
\hline miR-4330 & -0.0285087 & Down & 0.30886419 & 0.84601313 \\
\hline miR-133a-5p & -0.0284487 & Down & 0.24051057 & $0.80586|8|$ \\
\hline miR-2II0 & -0.0283739 & Down & 0.33449566 & 0.85489649 \\
\hline miR-6776-5p & -0.0283318 & Down & 0.39853903 & 0.87364718 \\
\hline miR-93-5p & -0.028325 & Down & 0.77082806 & 0.98290443 \\
\hline miR-4305 & -0.0282488 & Down & 0.45028902 & 0.88964976 \\
\hline$m i R-4 \mid I-3 p$ & -0.0282225 & Down & 0.61100763 & 0.94386479 \\
\hline miR-3189-5p & $-0.028 \mid 667$ & Down & 0.21137683 & 0.78263372 \\
\hline miR-6773-3p & $-0.028|4|$ & Down & 0.50601389 & 0.912181 \\
\hline$m i R-6762-5 p$ & -0.0280596 & Down & 0.32984973 & 0.85489649 \\
\hline miR-549a & -0.028046 & Down & 0.54673169 & 0.92261663 \\
\hline miR-3I50a-5p & -0.0280455 & Down & 0.32033013 & 0.85046325 \\
\hline miR-650 & -0.0279112 & Down & 0.27951311 & 0.83108541 \\
\hline
\end{tabular}

(Continued) 
Table 9 (Continued).

\begin{tabular}{|c|c|c|c|c|}
\hline miRNAs & Log2FoldChange & Regulation & P-value & P-adj \\
\hline miR-473I-5p & -0.0277768 & Down & 0.36275575 & 0.8588192 \\
\hline miR-4436a & -0.0276167 & Down & $0.3495337 \mid$ & 0.8588192 \\
\hline miR-639 & -0.0275526 & Down & 0.36815025 & 0.86195734 \\
\hline miR-548av-5p & -0.0275382 & Down & $0.33395 I 29$ & 0.85489649 \\
\hline miR-39|3-5p & -0.0274806 & Down & 0.42347246 & 0.8823614 \\
\hline miR-4662b & -0.0274059 & Down & 0.48890952 & 0.90656383 \\
\hline miR-45II & -0.027387 & Down & 0.55883847 & 0.92752708 \\
\hline miR-4650-5p & -0.0272772 & Down & 0.6722039 & 0.95648223 \\
\hline miR-4726-3p & $-0.02726 I I$ & Down & $0.4922006 I$ & 0.90695643 \\
\hline $\operatorname{miR}-3610$ & -0.0270911 & Down & 0.45237699 & 0.89093879 \\
\hline miR-2277-3p & -0.0270691 & Down & 0.55095919 & 0.92349191 \\
\hline miR-320I & -0.0270669 & Down & $0.2|4||4| 4$ & 0.78498335 \\
\hline miR-6I2 & -0.0269448 & Down & 0.52255245 & 0.91732227 \\
\hline miR-62I & -0.0269064 & Down & 0.42966946 & 0.8869482 \\
\hline miR-4503 & -0.0268456 & Down & 0.51622064 & 0.91376348 \\
\hline miR-19a-5p & -0.0268393 & Down & 0.41567275 & 0.8804535 \\
\hline miR-3939 & -0.0267855 & Down & 0.4278704 & 0.88394447 \\
\hline miR-6834-3p & -0.0267572 & Down & $0.4527078 I$ & 0.89093879 \\
\hline miR-3160-3p & $-0.026737 \mid$ & Down & 0.34553725 & 0.8588192 \\
\hline$m i R-548 j-3 p$ & -0.026724 & Down & 0.39225869 & $0.8728 \mid 803$ \\
\hline miR-4264 & -0.0267094 & Down & 0.36033208 & 0.8588192 \\
\hline miR-2II5-5p & -0.0266976 & Down & 0.46939468 & $0.893960 \mathrm{II}$ \\
\hline miR-6782-3p & -0.0266664 & Down & 0.50624103 & 0.912181 \\
\hline miR-4707-3p & -0.0266129 & Down & 0.6122267 & 0.94386479 \\
\hline$m i R-659-5 p$ & -0.0266029 & Down & 0.51175645 & 0.91376348 \\
\hline miR-I273c & -0.0265915 & Down & $0.5637 \mid 885$ & 0.92988283 \\
\hline miR-4778-3p & -0.026464 & Down & 0.56324824 & 0.92970327 \\
\hline miR-548a-5p & -0.0263646 & Down & 0.54053286 & 0.9213181 \\
\hline miR-8076 & -0.0263375 & Down & 0.3907435 I & $0.8728 \mid 803$ \\
\hline miR-6774-3p & -0.0262921 & Down & 0.21570366 & 0.7853205 \\
\hline miR-187-3p & -0.0262687 & Down & 0.61864354 & 0.94829596 \\
\hline miR-6780b-3p & -0.0262058 & Down & 0.55359073 & 0.92384946 \\
\hline miR-4654 & -0.0262048 & Down & 0.40631365 & 0.87475982 \\
\hline miR-4479 & -0.0261019 & Down & 0.40303872 & 0.87475982 \\
\hline miR-548ao-5p & -0.0260327 & Down & 0.36119964 & 0.8588192 \\
\hline miR-I229-3p & -0.0259448 & Down & 0.62982461 & 0.95065239 \\
\hline miR-4999-3p & -0.0259058 & Down & 0.51235821 & 0.91376348 \\
\hline miR-6794-5p & -0.0258639 & Down & 0.64968367 & 0.95321673 \\
\hline miR-5I8a-3p & -0.0258487 & Down & 0.46803021 & $0.8939601 \mathrm{I}$ \\
\hline miR-185-3p & -0.0257747 & Down & 0.58499315 & 0.93513803 \\
\hline miR-4667-3p & -0.0257646 & Down & 0.38641099 & $0.8696 / 208$ \\
\hline NCI_00000I97 & -0.0257608 & Down & $0.5366080 \mathrm{I}$ & 0.9213181 \\
\hline miR-200b-5p & -0.0257468 & Down & 0.28862915 & 0.83439474 \\
\hline$m i R-518 c-5 p$ & $-0.0257|5|$ & Down & 0.37392524 & 0.8649907 I \\
\hline$m i R-1911-5 p$ & -0.0257045 & Down & $0.4726898 I$ & 0.89396011 \\
\hline$m i R-36 \mid 3-5 p$ & -0.0256137 & Down & 0.52153217 & 0.91732227 \\
\hline miR-548y & -0.0255985 & Down & 0.36507913 & 0.8588192 \\
\hline miR-6755-5p & $-0.025567 \mid$ & Down & 0.39163753 & $0.8728 \mid 803$ \\
\hline miR-548as-5p & $-0.0254 \mid 77$ & Down & 0.34432168 & 0.8588192 \\
\hline
\end{tabular}

(Continued) 
Table 9 (Continued).

\begin{tabular}{|c|c|c|c|c|}
\hline miRNAs & Log2FoldChange & Regulation & P-value & P-adj \\
\hline miR-4468 & -0.0253343 & Down & 0.50380212 & 0.91147569 \\
\hline miR-4664-5p & -0.0252439 & Down & 0.52252164 & 0.91732227 \\
\hline miR-3|29-3p & -0.0252211 & Down & 0.48803069 & 0.90656383 \\
\hline miR-548ay-5p & $-0.025 \mid 434$ & Down & 0.52928404 & 0.92119547 \\
\hline miR-6770-3p & -0.0249958 & Down & 0.4626474 & 0.89396011 \\
\hline miR-I-5p & -0.024974 & Down & 0.54095921 & 0.9213181 \\
\hline miR-6807-3p & -0.0249379 & Down & 0.4241687 & $0.88236 \mid 4$ \\
\hline miR-7I60-3p & -0.0249049 & Down & 0.36478516 & 0.8588192 \\
\hline miR-934 & $-0.024895 I$ & Down & 0.36815835 & 0.86195734 \\
\hline miR-7|I2-5p & -0.0248867 & Down & 0.53293019 & 0.9213181 \\
\hline miR-8078 & -0.0247883 & Down & 0.36500255 & 0.8588192 \\
\hline$m i R-2 \mid 4-5 p$ & -0.0247315 & Down & 0.31406257 & 0.84917098 \\
\hline$m i R-68 \mid 4-5 p$ & -0.0247263 & Down & 0.48666242 & 0.90650744 \\
\hline$m i R-268 I-3 p$ & -0.0245218 & Down & 0.35525362 & 0.8588192 \\
\hline miR-4256 & -0.0245094 & Down & $0.364|37| 4$ & 0.8588192 \\
\hline miR-3619-5p & -0.0242098 & Down & 0.57698708 & 0.93390911 \\
\hline miR-6727-3p & $-0.024 \mid 848$ & Down & 0.46978366 & $0.8939601 \mathrm{I}$ \\
\hline$m i R-558 I-3 p$ & -0.024105 & Down & 0.43590749 & $0.8894 \mid 554$ \\
\hline$m i R-682 I-3 p$ & -0.0240699 & Down & 0.53920376 & 0.9213181 \\
\hline miR-5I9I & -0.0240566 & Down & 0.34090188 & 0.85516921 \\
\hline miR-6728-3p & -0.0240234 & Down & 0.61921683 & 0.94829596 \\
\hline NC2_00092197 & -0.0239201 & Down & 0.26857562 & $0.8286 \mid 865$ \\
\hline miR-I286 & -0.0238976 & Down & $0.4236|73|$ & 0.8823614 \\
\hline$m i R-548 e-5 p$ & -0.0238883 & Down & $0.48489 \mid 72$ & 0.90449982 \\
\hline miR-219a-I-3p & -0.0237329 & Down & 0.56052984 & 0.92768099 \\
\hline miR-64I & -0.0237023 & Down & 0.67361652 & 0.95648223 \\
\hline$m i R-145-5 p$ & -0.0236869 & Down & 0.9434724 & $0.998987 \mid 2$ \\
\hline$m i R-6744-5 p$ & -0.0236591 & Down & 0.54886022 & 0.92261663 \\
\hline$m i R-4649-5 p$ & $-0.023605 I$ & Down & 0.61179602 & 0.94386479 \\
\hline miR-3|7I & -0.0235034 & Down & 0.67350194 & 0.95648223 \\
\hline miR-149-3p & -0.023432 & Down & 0.39448524 & $0.8731074 \mid$ \\
\hline$m i R-4524 b-5 p$ & -0.0234286 & Down & 0.42410749 & 0.8823614 \\
\hline miR-I265 & -0.0233405 & Down & 0.4928787 I & 0.90695643 \\
\hline miR-7I52-3p & -0.023304 & Down & 0.57739485 & 0.93390911 \\
\hline miR-4720-3p & -0.0233017 & Down & 0.37809124 & $0.8649907 \mid$ \\
\hline miR-I537-5p & -0.0232765 & Down & 0.63968834 & 0.95096807 \\
\hline miR-4735-3p & -0.0232067 & Down & 0.38580088 & 0.86961208 \\
\hline$m i R-683 I-5 p$ & -0.0231041 & Down & 0.47232408 & $0.8939601 \mathrm{I}$ \\
\hline miR-302c-3p & -0.0230396 & Down & 0.36861128 & 0.86195734 \\
\hline miR-6I7 & -0.0229629 & Down & 0.72003158 & 0.96558066 \\
\hline miR-6877-3p & -0.0229476 & Down & 0.63855179 & 0.95096807 \\
\hline miR-5692a & -0.0229447 & Down & 0.423124 & 0.8823614 \\
\hline miR-640 & -0.0229348 & Down & 0.7008013 & 0.96268515 \\
\hline miR-3922-5p & -0.0228789 & Down & 0.29212106 & 0.83872374 \\
\hline$m i R-329-5 p$ & -0.0228768 & Down & 0.5137625 & 0.91376348 \\
\hline miR-4797-3p & -0.0228032 & Down & 0.33996273 & 0.85489649 \\
\hline miR-59I & -0.0227442 & Down & 0.51170058 & 0.91376348 \\
\hline$m i R-1255 b-2-3 p$ & -0.0227272 & Down & 0.64792269 & 0.95321673 \\
\hline miR-554 & -0.0226667 & Down & 0.5165602 & 0.91376348 \\
\hline
\end{tabular}

(Continued) 
Table 9 (Continued).

\begin{tabular}{|c|c|c|c|c|}
\hline miRNAs & Log2FoldChange & Regulation & P-value & P-adj \\
\hline miR-7850-5p & -0.0226549 & Down & $0.4208847 \mid$ & 0.8823614 \\
\hline miR-624-3p & -0.022569 & Down & 0.44792099 & 0.88961123 \\
\hline miR-50I-3p & $-0.02256 \mathrm{I}$ & Down & 0.72779336 & 0.96963657 \\
\hline miR-3I78 & -0.0225061 & Down & 0.35728742 & 0.8588192 \\
\hline miR-5II-3p & -0.0223247 & Down & 0.47990324 & 0.90289262 \\
\hline miR-4434 & -0.0222334 & Down & 0.481052 & 0.9030564 \\
\hline miR-6777-3p & -0.022166 & Down & 0.63978023 & 0.95096807 \\
\hline miR-1207-3p & -0.0219647 & Down & 0.54804246 & 0.92261663 \\
\hline miR-3|36-3p & -0.0219553 & Down & 0.61990964 & 0.94829596 \\
\hline miR-8080 & -0.0219457 & Down & 0.4413316 & 0.88952595 \\
\hline miR-6833-3p & -0.0218518 & Down & 0.61529953 & 0.94689808 \\
\hline miR-65I la-3p & -0.0218305 & Down & 0.62779774 & $0.949834 I$ \\
\hline miR-4782-3p & -0.021813 & Down & $0.389 \mid 3329$ & 0.87266367 \\
\hline miR-4759 & -0.021747 & Down & 0.47690937 & 0.89857557 \\
\hline miR-6788-3p & -0.0217395 & Down & 0.42326016 & 0.8823614 \\
\hline miR-4457 & -0.0217289 & Down & 0.35904329 & 0.8588192 \\
\hline$m i R-2114-3 p$ & -0.0217087 & Down & 0.46680529 & $0.893960 \mathrm{II}$ \\
\hline miR-208a-3p & -0.0216887 & Down & $0.5737|99|$ & 0.93390911 \\
\hline miR-924 & -0.0216297 & Down & 0.48498084 & 0.90449982 \\
\hline miR-68।9-5p & -0.0216207 & Down & $0.60973|3|$ & 0.94386479 \\
\hline miR-4782-5p & -0.0216032 & Down & 0.53467106 & 0.9213181 \\
\hline miR-6753-5p & -0.0215961 & Down & $0.5|328|$ & 0.91376348 \\
\hline miR-4779 & -0.0215243 & Down & 0.35563879 & 0.8588192 \\
\hline miR-6847-5p & -0.0214709 & Down & $0.493|4| 74$ & 0.90695643 \\
\hline miR-302b-3p & -0.0213073 & Down & $0.464344 I I$ & $0.893960 \mathrm{II}$ \\
\hline miR-583 & -0.0212315 & Down & 0.56736377 & 0.9303065 \\
\hline miR-6878-3p & -0.0212105 & Down & 0.36926655 & 0.86195734 \\
\hline miR-548ag & $-0.02 I 2064$ & Down & 0.51578333 & 0.91376348 \\
\hline miR-3।94-5p & -0.021182 & Down & $0.702348 I I$ & 0.96268515 \\
\hline miR-6828-5p & -0.0211656 & Down & 0.39486429 & $0.8731074 \mid$ \\
\hline miR-7I09-5p & -0.0211595 & Down & 0.50943748 & 0.91230009 \\
\hline miR-196b-3p & -0.0210447 & Down & $0.55285|4|$ & 0.92384946 \\
\hline miR-662 & -0.0210389 & Down & 0.71800577 & 0.96505269 \\
\hline miR-5I92 & -0.020993 & Down & $0.46504 \mid 84$ & $0.8939601 \mathrm{I}$ \\
\hline miR-1266-5p & -0.0209397 & Down & 0.55339061 & 0.92384946 \\
\hline$m i R-548 f-5 p$ & -0.020819 & Down & 0.60725348 & 0.94355588 \\
\hline miR-89la-5p & -0.0206623 & Down & $0.506 \mid 4706$ & 0.912181 \\
\hline$m i R-5 \mid 4 a-5 p$ & -0.0203138 & Down & 0.49325699 & 0.90695643 \\
\hline miR-567 & -0.020309 I & Down & 0.60896204 & 0.94386479 \\
\hline miR-5I9d-5p & -0.0203012 & Down & 0.51104339 & 0.91376348 \\
\hline miR-6742-5p & -0.0200957 & Down & 0.53027998 & 0.9213181 \\
\hline miR-5087 & -0.0200794 & Down & 0.52357825 & $0.917869 \mid$ \\
\hline miR-16-I-3p & -0.0200778 & Down & $0.33434|4|$ & 0.85489649 \\
\hline miR-3202 & -0.0200496 & Down & 0.52892652 & 0.92119547 \\
\hline miR-6888-5p & $-0.02004 \mid 8$ & Down & 0.46309567 & 0.89396011 \\
\hline miR-2276-5p & -0.0200027 & Down & 0.47050027 & $0.893960 \mathrm{II}$ \\
\hline miR-37I4 & -0.0199413 & Down & 0.45375523 & 0.89093879 \\
\hline miR-5708 & -0.0198854 & Down & 0.48209859 & 0.9030564 \\
\hline miR-208b-3p & -0.0198438 & Down & 0.43849767 & 0.88952595 \\
\hline
\end{tabular}

(Continued) 
Table 9 (Continued).

\begin{tabular}{|c|c|c|c|c|}
\hline miRNAs & Log2FoldChange & Regulation & P-value & P-adj \\
\hline miR-8070 & -0.0198259 & Down & 0.56288068 & 0.92970327 \\
\hline miR-4524a-5p & $-0.019750 \mid$ & Down & 0.53816235 & 0.9213181 \\
\hline miR-3189-3p & -0.0196763 & Down & $0.4669157 \mid$ & 0.89396011 \\
\hline miR-4695-5p & -0.0196329 & Down & 0.72755483 & 0.96963657 \\
\hline$m i R-519 d-3 p$ & -0.0196262 & Down & 0.51976908 & 0.91632037 \\
\hline miR-39I8 & -0.0196255 & Down & $0.4|13765|$ & 0.8793356 \\
\hline miR-6883-3p & -0.0195894 & Down & 0.64183749 & 0.95128163 \\
\hline miR-298 & -0.0195803 & Down & 0.55851468 & 0.92752708 \\
\hline miR-5095 & -0.0194938 & Down & 0.59415038 & 0.93829717 \\
\hline miR-373-3p & -0.0194035 & Down & 0.47647192 & 0.89841001 \\
\hline miR-3622b-3p & -0.0193703 & Down & 0.67771735 & 0.95648223 \\
\hline $\mathrm{miR}-3656$ & $-0.019348 \mid$ & Down & 0.82855543 & 0.99440018 \\
\hline miR-4265 & -0.019335 & Down & 0.44210537 & 0.88952595 \\
\hline miR-589-3p & -0.0192968 & Down & 0.6412559 & 0.95096807 \\
\hline$m i R-6722-3 p$ & -0.0192565 & Down & 0.43605587 & $0.8894 \mid 554$ \\
\hline miR-I248 & -0.0192199 & Down & 0.48145878 & 0.9030564 \\
\hline miR-807I & -0.0191373 & Down & $0.5794 I 186$ & 0.93436563 \\
\hline miR-6822-3p & -0.0190523 & Down & 0.53678876 & 0.9213181 \\
\hline$m i R-3689 d$ & -0.0190456 & Down & 0.4142069 & 0.8804535 \\
\hline miR-548ar-3p & -0.0189736 & Down & 0.55852155 & 0.92752708 \\
\hline$m i R-548 d-5 p$ & -0.0189594 & Down & 0.4817617 & 0.9030564 \\
\hline miR-6802-3p & -0.0189422 & Down & 0.51874876 & 0.91564857 \\
\hline $\mathrm{miR}-4504$ & -0.0189187 & Down & 0.49111452 & 0.90695643 \\
\hline miR-6864-3p & -0.0188964 & Down & 0.54207416 & 0.92199245 \\
\hline $\operatorname{miR}-3618$ & -0.0187864 & Down & 0.69179636 & 0.96077402 \\
\hline miR-103b & -0.0187592 & Down & $0.5739581 \mathrm{I}$ & 0.93390911 \\
\hline miR-6844 & -0.018755 & Down & 0.77362276 & $0.985238 I$ \\
\hline$m i R-43 I-3 p$ & -0.0187068 & Down & 0.79929465 & 0.98820926 \\
\hline miR-I 200 & -0.018706 & Down & $0.567582 \mathrm{II}$ & 0.9303065 \\
\hline miR-7II0-3p & -0.0186295 & Down & 0.46493742 & 0.89396011 \\
\hline$m i R-5004-5 p$ & -0.0186198 & Down & 0.40300059 & 0.87475982 \\
\hline miR-3617-3p & -0.0186036 & Down & 0.66555667 & 0.95557578 \\
\hline miR-6779-5p & -0.0185894 & Down & 0.51792705 & 0.91482647 \\
\hline$m i R-454-3 p$ & -0.018482 & Down & 0.76305689 & 0.98140055 \\
\hline miR-5688 & -0.0183095 & Down & 0.54890305 & 0.92261663 \\
\hline miR-4777-3p & -0.0182984 & Down & $0.5844 \mid 507$ & 0.93513803 \\
\hline miR-I38-I-3p & -0.0182606 & Down & 0.72136765 & 0.96558066 \\
\hline miR-467I-3p & -0.0182268 & Down & 0.69455307 & 0.96174643 \\
\hline miR-4463 & -0.0182222 & Down & 0.48413304 & 0.90449982 \\
\hline miR-4424 & -0.0181867 & Down & 0.56815021 & 0.9303065 \\
\hline miR-1268b & -0.0179837 & Down & 0.8287958 & 0.99440018 \\
\hline$m i R-4732-3 p$ & -0.0179263 & Down & 0.58509804 & 0.93513803 \\
\hline$m i R-6746-5 p$ & -0.0179198 & Down & $0.58247 \mid 49$ & 0.93436563 \\
\hline$m i R-6892-5 p$ & -0.017886 & Down & 0.59755189 & 0.93869704 \\
\hline$m i R-38 I-5 p$ & -0.0178605 & Down & 0.65525257 & 0.95321673 \\
\hline miR-I26I & -0.0178075 & Down & 0.48991008 & 0.90695643 \\
\hline$m i R-6 I 6-3 p$ & -0.0177903 & Down & 0.47341623 & 0.89396011 \\
\hline miR-4676-3p & $-0.017780 I$ & Down & $0.6744 \mid 895$ & 0.95648223 \\
\hline$m i R-4645-3 p$ & -0.017748 & Down & 0.64695212 & 0.95321673 \\
\hline
\end{tabular}

(Continued) 
Table 9 (Continued).

\begin{tabular}{|c|c|c|c|c|}
\hline miRNAs & Log2FoldChange & Regulation & P-value & P-adj \\
\hline miR-4293 & -0.0177317 & Down & 0.66240796 & 0.95510802 \\
\hline$m i R-688 I-3 p$ & -0.0177258 & Down & 0.76287968 & $0.98 \mid 40055$ \\
\hline miR-744-3p & -0.0176999 & Down & $0.71123,168$ & 0.96505269 \\
\hline miR-216b-3p & -0.0176221 & Down & 0.51523309 & 0.91376348 \\
\hline miR-3622a-3p & -0.0175364 & Down & 0.57580661 & 0.93390911 \\
\hline miR-39I9 & $-0.017507 \mid$ & Down & 0.5510952 & 0.92349191 \\
\hline miR-470I-3p & $-0.01748 \mid 8$ & Down & 0.44866694 & 0.88964976 \\
\hline miR-3|3| & -0.0174639 & Down & 0.52596692 & 0.91954761 \\
\hline miR-4760-5p & -0.0173869 & Down & 0.67234291 & 0.95648223 \\
\hline miR-4433b-3p & -0.0172945 & Down & 0.54300728 & 0.9222099 \\
\hline$m i R-476 I-5 p$ & -0.0172715 & Down & 0.5875354 & 0.93675929 \\
\hline miR-892a & -0.0172692 & Down & 0.65506354 & 0.95321673 \\
\hline miR-345-3p & -0.0172563 & Down & 0.64008036 & 0.95096807 \\
\hline miR-3I47 & -0.0172107 & Down & $0.6406645 I$ & 0.95096807 \\
\hline miR-378h & -0.0171729 & Down & $0.5046 \mid 254$ & 0.91199313 \\
\hline miR-689I-3p & $-0.0|7| 45 \mid$ & Down & 0.76246977 & $0.98 \mid 40055$ \\
\hline miR-188-3p & -0.0170843 & Down & 0.65777789 & 0.95321673 \\
\hline miR-2277-5p & $-0.017066 \mid$ & Down & 0.56519544 & 0.9303065 \\
\hline miR-7I08-3p & -0.0170624 & Down & 0.63080393 & 0.95086097 \\
\hline miR-6850-5p & -0.016963 & Down & 0.81070492 & 0.99064883 \\
\hline miR-767-3p & -0.0169514 & Down & $0.7569 \mid 552$ & $0.98 \mid 40055$ \\
\hline$m i R-345-5 p$ & -0.0168958 & Down & 0.81256332 & 0.99064883 \\
\hline miR-6889-5p & -0.0168908 & Down & 0.37711739 & 0.8649907 I \\
\hline miR-I45-3p & -0.0168628 & Down & 0.70862699 & 0.96360966 \\
\hline miR-4802-3p & -0.0168272 & Down & $0.5732830 \mathrm{I}$ & 0.93390911 \\
\hline miR-376a-2-5p & -0.0168259 & Down & 0.59327345 & 0.93770773 \\
\hline miR-6I33 & -0.0167772 & Down & $0.5089 \mid 359$ & 0.91230009 \\
\hline$m i R-469 I-3 p$ & -0.0165958 & Down & 0.50664374 & 0.912181 \\
\hline miR-4783-5p & -0.0165943 & Down & 0.67050849 & 0.95648223 \\
\hline miR-45I4 & -0.0165234 & Down & 0.54631268 & 0.92261663 \\
\hline miR-6745 & -0.0165196 & Down & 0.52870674 & 0.92119547 \\
\hline miR-548b-3p & -0.0164887 & Down & $0.56978 \mid 74$ & 0.93210635 \\
\hline miR-4435 & -0.0164779 & Down & 0.56561035 & 0.9303065 \\
\hline miR-3I65 & -0.0164702 & Down & 0.5816504 & 0.93436563 \\
\hline$m i R-219 b-5 p$ & -0.0164568 & Down & 0.59560483 & 0.9386847 I \\
\hline miR-548v & -0.0164565 & Down & 0.70844026 & 0.96360966 \\
\hline miR-2467-3p & -0.0164015 & Down & 0.57742474 & 0.93390911 \\
\hline miR-3924 & -0.0162979 & Down & 0.70061417 & 0.96268515 \\
\hline miR-4766-5p & -0.016287 & Down & 0.48436282 & 0.90449982 \\
\hline miR-4673 & -0.0162448 & Down & 0.59710592 & 0.9386847 I \\
\hline$m i R-65 \mid 3-3 p$ & -0.0162307 & Down & 0.60967682 & 0.94386479 \\
\hline miR-465I & -0.0160382 & Down & $0.8276 \mid 866$ & 0.99438053 \\
\hline$m i R-485-3 p$ & -0.0160167 & Down & 0.87943283 & $0.998987 / 2$ \\
\hline miR-4662a-3p & -0.015971 & Down & 0.49625711 & 0.90968672 \\
\hline$m i R-6845-3 p$ & -0.0158811 & Down & 0.67837672 & 0.95648223 \\
\hline miR-3I44-5p & -0.015822 & Down & 0.53056015 & 0.9213181 \\
\hline miR-II 84 & -0.0158072 & Down & 0.59828657 & 0.93870358 \\
\hline miR-6839-5p & $-0.0|5655|$ & Down & 0.6119096 & 0.94386479 \\
\hline miR-I 258 & -0.015538 & Down & 0.58766102 & 0.93675929 \\
\hline
\end{tabular}

(Continued) 
Table 9 (Continued).

\begin{tabular}{|c|c|c|c|c|}
\hline miRNAs & Log2FoldChange & Regulation & P-value & P-adj \\
\hline miR-5697 & -0.0154575 & Down & 0.68886204 & 0.96036263 \\
\hline miR-8065 & -0.015457 & Down & 0.74994003 & 0.97742837 \\
\hline miR-6870-5p & -0.0154516 & Down & $0.66323 \mid 38$ & 0.95510802 \\
\hline miR-206 & -0.0153486 & Down & 0.55904258 & 0.92752708 \\
\hline miR-4452 & -0.0153427 & Down & 0.58344668 & 0.93436563 \\
\hline miR-5I0-3p & -0.0153235 & Down & 0.66778315 & 0.95631006 \\
\hline miR-146b-3p & -0.0153142 & Down & 0.57687517 & 0.93390911 \\
\hline miR-45I3 & -0.0152798 & Down & 0.6014067 & 0.93953261 \\
\hline miR-7847-3p & $-0.015276 \mid$ & Down & 0.77823881 & 0.985258 \\
\hline miR-6739-5p & -0.0152297 & Down & $0.776|263|$ & 0.985258 \\
\hline$m i R-4646-3 p$ & -0.0151834 & Down & 0.84117716 & 0.99654281 \\
\hline miR-2।I6-5p & -0.0151732 & Down & 0.6849632 & 0.95932176 \\
\hline miR-68I7-3p & -0.0150789 & Down & 0.61008554 & 0.94386479 \\
\hline miR-4660 & -0.0150254 & Down & 0.50800372 & 0.91230009 \\
\hline miR-3।60-5p & -0.0149645 & Down & 0.67722872 & 0.95648223 \\
\hline miR-50II-5p & -0.0149335 & Down & 0.59054265 & 0.93675929 \\
\hline miR-5685 & -0.0149228 & Down & 0.59141154 & 0.93707007 \\
\hline miR-4729 & -0.0149005 & Down & 0.74047795 & 0.97402999 \\
\hline miR-585-3p & -0.0148846 & Down & 0.741324 & 0.97402999 \\
\hline miR-3I8I & -0.0148665 & Down & 0.57151784 & 0.93363213 \\
\hline miR-7I59-5p & -0.0148501 & Down & 0.60210514 & 0.93953261 \\
\hline miR-1236-5p & -0.0148479 & Down & 0.73719766 & 0.97354829 \\
\hline$m i R-655-3 p$ & -0.0148337 & Down & 0.67655901 & 0.95648223 \\
\hline miR-6773-5p & -0.0148148 & Down & 0.63769818 & 0.95096807 \\
\hline$m i R-452-3 p$ & -0.0146011 & Down & 0.62426449 & 0.9493253 \\
\hline miR-4532 & -0.0145244 & Down & 0.79484255 & 0.98759926 \\
\hline miR-I 276 & -0.0145182 & Down & 0.66431616 & 0.95510802 \\
\hline$m i R-495-5 p$ & -0.014511 & Down & 0.64839579 & 0.95321673 \\
\hline miR-3।84-5p & -0.0144988 & Down & 0.67280274 & 0.95648223 \\
\hline miR-6873-5p & $-0.0|4497|$ & Down & 0.63738698 & 0.95096807 \\
\hline miR-1237-5p & -0.014493 & Down & 0.63864074 & 0.95096807 \\
\hline$m i R-605-5 p$ & -0.0144929 & Down & 0.64097092 & 0.95096807 \\
\hline miR-548b-5p & $-0.01446 \mid 4$ & Down & 0.76570467 & 0.98147681 \\
\hline miR-7856-5p & -0.0143765 & Down & 0.72382632 & 0.96691758 \\
\hline miR-5582-3p & -0.0143693 & Down & 0.65392278 & 0.95321673 \\
\hline miR-6786-3p & -0.0143516 & Down & 0.6934944 & 0.96137172 \\
\hline miR-4723-3p & -0.0140957 & Down & 0.88560625 & $0.998987 \mid 2$ \\
\hline$m i R-6855-5 p$ & -0.0140764 & Down & 0.68879875 & 0.96036263 \\
\hline miR-I46a-3p & -0.0140732 & Down & 0.63408847 & 0.95096807 \\
\hline miR-4726-5p & -0.0140357 & Down & $0.6203480 I$ & 0.94829596 \\
\hline miR-6782-5p & -0.0140077 & Down & 0.4999732 & 0.91121111 \\
\hline miR-I 278 & -0.013964 & Down & 0.81623945 & 0.99127826 \\
\hline miR-346 & -0.0139568 & Down & 0.57180427 & 0.93363213 \\
\hline miR-190b & $-0.013945 \mid$ & Down & 0.55975316 & 0.9276427 \\
\hline miR-887-3p & -0.0138433 & Down & 0.80365516 & 0.99063213 \\
\hline $\mathrm{miR}-6068$ & -0.0137686 & Down & 0.82973935 & 0.99448905 \\
\hline miR-63I & -0.0137437 & Down & $0.67728 \mid 43$ & 0.95648223 \\
\hline miR-6737-5p & -0.0137008 & Down & 0.74839715 & 0.97742837 \\
\hline miR-7702 & $-0.0|3645|$ & Down & 0.68775489 & 0.96036263 \\
\hline
\end{tabular}

(Continued) 
Table 9 (Continued).

\begin{tabular}{|c|c|c|c|c|}
\hline miRNAs & Log2FoldChange & Regulation & P-value & P-adj \\
\hline miR-4709-3p & -0.0135279 & Down & 0.57658086 & 0.93390911 \\
\hline miR-6874-3p & -0.0135163 & Down & 0.59284104 & 0.93770773 \\
\hline miR-6846-5p & -0.0134762 & Down & 0.6527056 & 0.95321673 \\
\hline miR-2278 & -0.0133521 & Down & $0.57674 I$ & 0.93390911 \\
\hline miR-130a-5p & -0.013298 & Down & 0.70637716 & 0.96360966 \\
\hline miR-6836-5p & -0.0131042 & Down & 0.54763293 & 0.92261663 \\
\hline miR-6882-5p & -0.0130684 & Down & 0.5242369 & 0.91825968 \\
\hline miR-3193 & $-0.013058 \mid$ & Down & 0.59827391 & 0.93870358 \\
\hline miR-68I2-3p & -0.013037 & Down & 0.826037 & 0.99340905 \\
\hline miR-6819-3p & -0.012997 & Down & 0.80469099 & 0.99064883 \\
\hline miR-6857-5p & -0.0129467 & Down & 0.69145376 & 0.96077402 \\
\hline miR-4655-5p & $-0.012925 \mid$ & Down & $0.687874 \mid$ & 0.96036263 \\
\hline miR-44I8 & -0.0129231 & Down & $0.532 \mid 4975$ & 0.9213181 \\
\hline miR-8066 & -0.0128877 & Down & 0.79360278 & 0.98694309 \\
\hline miR-I28-I-5p & -0.0128078 & Down & 0.87264725 & 0.99898712 \\
\hline miR-470I-5p & -0.0127842 & Down & 0.75883109 & $0.98 \mid 40055$ \\
\hline miR-6750-3p & -0.0127744 & Down & 0.70392756 & 0.96360966 \\
\hline miR-I30b-5p & -0.0127465 & Down & $0.7575|85|$ & $0.98 \mid 40055$ \\
\hline miR-3653-5p & -0.0127035 & Down & $0.797|568|$ & 0.98759926 \\
\hline miR-I I99-5p & -0.0126985 & Down & 0.71040964 & 0.96505269 \\
\hline miR-548s & $-0.012665 \mid$ & Down & 0.74107714 & 0.97402999 \\
\hline miR-4799-5p & -0.0126574 & Down & 0.70111791 & 0.96268515 \\
\hline miR-665 & -0.0126447 & Down & 0.69642239 & 0.96226104 \\
\hline miR-548d-3p & -0.0126426 & Down & 0.71618248 & 0.96505269 \\
\hline miR-5047 & -0.0126237 & Down & $0.653684 I$ & 0.95321673 \\
\hline miR-2I4-3p & -0.0125973 & Down & 0.66824257 & 0.95631006 \\
\hline miR-5007-5p & -0.0125917 & Down & $0.645 \mid 4439$ & 0.95321673 \\
\hline miR-6824-5p & $-0.0|2555|$ & Down & 0.77703408 & 0.985258 \\
\hline miR-6835-5p & -0.0125379 & Down & 0.66382121 & 0.95510802 \\
\hline miR-3976 & -0.0125279 & Down & 0.67920619 & 0.9566446 \\
\hline miR-5706 & -0.0125053 & Down & 0.69376627 & $0.96137 \mid 72$ \\
\hline miR-187-5p & -0.0124375 & Down & 0.72563224 & 0.96826128 \\
\hline miR-557I-5p & -0.0124326 & Down & 0.8556283 & 0.99898712 \\
\hline miR-6862-3p & -0.0124127 & Down & 0.74999562 & 0.97742837 \\
\hline miR-2276-3p & -0.0123549 & Down & 0.65208401 & 0.95321673 \\
\hline miR-5579-3p & -0.0123529 & Down & 0.6752284 & 0.95648223 \\
\hline miR-650I-5p & -0.0123046 & Down & 0.61453806 & 0.94686019 \\
\hline miR-183-5p & -0.0122913 & Down & 0.761594 & $0.98 \mid 40055$ \\
\hline miR-3I23 & -0.012244 & Down & 0.66679721 & 0.95631006 \\
\hline miR-519e-5p & -0.0122324 & Down & 0.58087819 & 0.93436563 \\
\hline miR-5587-5p & -0.0122294 & Down & $0.65|9779|$ & 0.95321673 \\
\hline miR-4423-5p & -0.0121935 & Down & 0.73278235 & 0.9725372 \\
\hline miR-378c & -0.0121663 & Down & $0.6500880 I$ & 0.95321673 \\
\hline miR-4758-5p & -0.0121174 & Down & 0.77102542 & 0.98290443 \\
\hline miR-I468-3p & -0.0120833 & Down & 0.78243923 & 0.98667434 \\
\hline miR-7855-5p & -0.0120272 & Down & 0.76376796 & $0.98 \mid 40055$ \\
\hline miR-4647 & -0.0119836 & Down & 0.7564745 I & $0.98 \mid 40055$ \\
\hline miR-506-5p & -0.0119826 & Down & 0.74052913 & 0.97402999 \\
\hline miR-4776-3p & -0.0119525 & Down & 0.71186538 & 0.96505269 \\
\hline
\end{tabular}

(Continued) 
Table 9 (Continued).

\begin{tabular}{|c|c|c|c|c|}
\hline miRNAs & Log2FoldChange & Regulation & P-value & P-adj \\
\hline miR-3666 & -0.0119274 & Down & 0.58255769 & 0.93436563 \\
\hline miR-6827-5p & -0.011866 & Down & 0.68812192 & 0.96036263 \\
\hline miR-6790-3p & -0.011811 & Down & 0.7844565 & 0.98667434 \\
\hline miR-5583-3p & -0.0|| $797 \mid$ & Down & 0.79156515 & 0.98667434 \\
\hline miR-I295b-5p & -0.0117206 & Down & 0.6919816 & 0.96077402 \\
\hline miR-3677-3p & -0.0117104 & Down & 0.67769257 & 0.95648223 \\
\hline $\operatorname{miR}-4527$ & -0.0116914 & Down & 0.57519673 & 0.93390911 \\
\hline miR-3II5 & $-0.0|1647|$ & Down & 0.79078548 & 0.98667434 \\
\hline miR-143-5p & -0.0116368 & Down & 0.73628333 & 0.97354829 \\
\hline miR-4637 & -0.0115636 & Down & 0.71639324 & 0.96505269 \\
\hline miR-II 82 & -0.0114596 & Down & 0.74557094 & $0.976409 \mid$ \\
\hline$m i R-548 t-5 p$ & -0.0114263 & Down & 0.72567052 & 0.96826128 \\
\hline miR-4260 & -0.0114243 & Down & 0.71608133 & 0.96505269 \\
\hline$m i R-520 g-5 p$ & -0.0113337 & Down & 0.71718692 & 0.96505269 \\
\hline miR-605-3p & -0.0113136 & Down & 0.68422057 & 0.95880418 \\
\hline miR-873-3p & $-0.01|292|$ & Down & 0.73299788 & 0.9725372 \\
\hline miR-I 244 & -0.0112603 & Down & 0.6232706 & $0.9492584 \mid$ \\
\hline miR-8055 & -0.0109754 & Down & 0.66758111 & 0.95631006 \\
\hline miR-3662 & -0.0109397 & Down & 0.69726598 & 0.96239183 \\
\hline$m i R-6862-5 p$ & $-0.01084 \mid$ & Down & 0.72132034 & 0.96558066 \\
\hline miR-4658 & -0.0108322 & Down & 0.70165965 & $0.962685 I 5$ \\
\hline miR-6767-3p & -0.0108203 & Down & 0.74011199 & 0.97402999 \\
\hline miR-99a-5p & -0.0107907 & Down & 0.96731243 & $0.998987 \mid 2$ \\
\hline miR-6768-3p & -0.0106522 & Down & 0.78842766 & 0.98667434 \\
\hline miR-4659a-5p & $-0.010645 \mid$ & Down & 0.79792473 & 0.98759926 \\
\hline miR-2III & -0.0106433 & Down & $0.7750 \mid 288$ & 0.985258 \\
\hline miR-6735-5p & -0.0105937 & Down & $0.683|904|$ & 0.95853044 \\
\hline miR-3I35a & -0.0105893 & Down & 0.69622559 & 0.96226104 \\
\hline $\operatorname{miR}-4295$ & -0.010552 & Down & 0.72600758 & 0.96826128 \\
\hline miR-7107-3p & -0.0105176 & Down & 0.84262259 & $0.9965428 \mathrm{I}$ \\
\hline miR-128-2-5p & -0.0105073 & Down & 0.66830072 & 0.95631006 \\
\hline miR-6859-5p & -0.0104776 & Down & $0.750787 / 2$ & 0.97770354 \\
\hline miR-3684 & -0.0104013 & Down & 0.83539086 & $0.9965428 \mathrm{I}$ \\
\hline miR-30d-3p & -0.0103853 & Down & 0.71051853 & 0.96505269 \\
\hline$m i R-4638-5 p$ & -0.0103425 & Down & 0.70833184 & 0.96360966 \\
\hline miR-4789-3p & -0.0103283 & Down & $0.746 \mid 3358$ & $0.976409 \mid$ \\
\hline miR-190a-3p & -0.0102881 & Down & 0.84773027 & $0.9965428 \mathrm{I}$ \\
\hline miR-3689f & -0.0101659 & Down & 0.70864679 & 0.96360966 \\
\hline miR-3913-3p & -0.0101642 & Down & 0.72958913 & 0.97152542 \\
\hline$m i R-4633-3 p$ & -0.0101254 & Down & 0.85388096 & 0.99898712 \\
\hline$m i R-486-5 p$ & -0.0099604 & Down & 0.95866057 & $0.998987 \mid 2$ \\
\hline miR-6747-3p & -0.009957 & Down & $0.64086|7|$ & 0.95096807 \\
\hline$m i R-125 I-3 p$ & -0.0099478 & Down & 0.74176683 & $0.974 \mid I 382$ \\
\hline miR-10a-3p & -0.0098892 & Down & 0.78024065 & 0.98667434 \\
\hline miR-658 & -0.0098766 & Down & 0.7897364 & 0.98667434 \\
\hline miR-944 & -0.0098706 & Down & 0.81797369 & $0.99 \mid 27826$ \\
\hline$m i R-47 \mid 3-5 p$ & -0.0098246 & Down & 0.83457819 & 0.99622199 \\
\hline miR-5006-3p & -0.009715 & Down & 0.78606427 & 0.98667434 \\
\hline miR-373-5p & -0.0095526 & Down & 0.73271645 & 0.9725372 \\
\hline
\end{tabular}

(Continued) 
Table 9 (Continued).

\begin{tabular}{|c|c|c|c|c|}
\hline miRNAs & Log2FoldChange & Regulation & P-value & P-adj \\
\hline $\operatorname{miR}-3169$ & -0.0095199 & Down & $0.7|7| 234 \mid$ & 0.96505269 \\
\hline miR-5I8a-5p & $-0.00948 I I$ & Down & $0.769 \mid 8477$ & 0.98237037 \\
\hline miR-464I & -0.0094703 & Down & 0.81044806 & 0.99064883 \\
\hline miR-3692-5p & $-0.0093 \mid 47$ & Down & 0.76433074 & $0.98 \mid 40055$ \\
\hline miR-3 $126-5 p$ & -0.0093056 & Down & $0.656 \mid 4493$ & 0.95321673 \\
\hline miR-576-3p & -0.009283 & Down & 0.78846763 & 0.98667434 \\
\hline miR-I285-5p & -0.0092817 & Down & 0.7864442 & 0.98667434 \\
\hline miR-4784 & -0.0092731 & Down & $0.768027 \mid 8$ & 0.98237037 \\
\hline miR-498 & -0.009158 & Down & 0.69256634 & 0.96106669 \\
\hline miR-219a-2-3p & -0.0091545 & Down & 0.82074845 & 0.99168948 \\
\hline miR-450b-3p & -0.0090465 & Down & $0.8524484 I$ & 0.99898712 \\
\hline$m i R-615-3 p$ & -0.0090186 & Down & 0.80687156 & 0.99064883 \\
\hline miR-657 & -0.009012 & Down & 0.82272197 & 0.99252298 \\
\hline miR-50II-3p & -0.0089942 & Down & 0.70445092 & 0.96360966 \\
\hline miR-4792 & -0.008984 & Down & 0.83702003 & $0.9965428 \mathrm{I}$ \\
\hline miR-3944-3p & -0.0089807 & Down & 0.81317265 & 0.99092163 \\
\hline miR-4769-3p & -0.0089698 & Down & 0.92653983 & 0.99898712 \\
\hline miR-6872-5p & -0.0089365 & Down & 0.66920104 & 0.95648223 \\
\hline miR-4727-3p & -0.008894 & Down & 0.78361029 & 0.98667434 \\
\hline miR-6876-5p & -0.0088936 & Down & 0.75998162 & $0.98 \mid 40055$ \\
\hline miR-205-5p & -0.0088756 & Down & 0.6575788 & 0.95321673 \\
\hline$m i R-494-5 p$ & $-0.008859 \mid$ & Down & 0.81959201 & 0.99127826 \\
\hline miR-2355-3p & -0.008706 & Down & 0.85986073 & 0.99898712 \\
\hline miR-4474-3p & -0.0086358 & Down & 0.7670933 & 0.98227692 \\
\hline miR-7852-3p & -0.008608 & Down & $0.735 \mid 3737$ & 0.97354829 \\
\hline miR-383-5p & -0.0085958 & Down & 0.85496752 & 0.99898712 \\
\hline miR-875-5p & -0.0085243 & Down & 0.8839679 & 0.99898712 \\
\hline miR-6822-5p & -0.0082844 & Down & $0.8190 \mid 427$ & $0.99 \mid 27826$ \\
\hline miR-569I & -0.0082025 & Down & 0.78963006 & 0.98667434 \\
\hline miR-7I55-3p & -0.0081525 & Down & 0.79710014 & 0.98759926 \\
\hline miR-4448 & -0.0081318 & Down & $0.7|75038|$ & 0.96505269 \\
\hline miR-3।82 & -0.0080719 & Down & 0.7367675 & 0.97354829 \\
\hline miR-5680 & -0.0080707 & Down & 0.8196367 & $0.99 \mid 27826$ \\
\hline miR-654-5p & -0.0080072 & Down & 0.87347525 & 0.99898712 \\
\hline miR-4794 & -0.007985 I & Down & 0.81435699 & 0.99122915 \\
\hline miR-4772-5p & -0.0079826 & Down & 0.80753955 & 0.99064883 \\
\hline miR-100-5p & $-0.007834 \mid$ & Down & 0.98391904 & 0.99898712 \\
\hline let-7g-3p & -0.0077228 & Down & 0.75985452 & $0.98 \mid 40055$ \\
\hline miR-4645-5p & -0.0076272 & Down & $0.828 \mid 2993$ & 0.99440018 \\
\hline miR-188-5p & $-0.0076 \mid 42$ & Down & 0.87848853 & 0.99898712 \\
\hline miR-338-5p & $-0.0076 \mid 22$ & Down & 0.81671067 & $0.99 \mid 27826$ \\
\hline miR-I 256 & -0.0076115 & Down & $0.843|I 54|$ & $0.9965428 I$ \\
\hline miR-708-5p & -0.0075769 & Down & 0.80679807 & 0.99064883 \\
\hline miR-6789-5p & -0.0075666 & Down & 0.92606518 & 0.99898712 \\
\hline miR-6787-3p & -0.0075105 & Down & 0.89165272 & 0.99898712 \\
\hline miR-68I5-5p & -0.0074763 & Down & $0.816 \mid 4637$ & $0.99 \mid 27826$ \\
\hline miR-7I8 & -0.0074274 & Down & 0.83027884 & 0.99448905 \\
\hline miR-3622a-5p & -0.0073929 & Down & $0.83 \mid 49808$ & 0.99505896 \\
\hline$m i R-629-3 p$ & -0.0073902 & Down & $0.9044 \mid 834$ & 0.99898712 \\
\hline
\end{tabular}

(Continued) 
Table 9 (Continued).

\begin{tabular}{|c|c|c|c|c|}
\hline miRNAs & Log2FoldChange & Regulation & P-value & P-adj \\
\hline$m i R-4318$ & $-0.007 \mid 434$ & Down & 0.79009082 & 0.98667434 \\
\hline miR-I224-5p & $-0.007 \mid 228$ & Down & 0.89904345 & $0.998987 \mid 2$ \\
\hline$m i R-368 I-5 p$ & $-0.007|2| 2$ & Down & 0.85999628 & $0.998987 \mid 2$ \\
\hline miR-5580-5p & -0.0070837 & Down & 0.87394584 & $0.998987 \mid 2$ \\
\hline miR-767-5p & $-0.00708 \mid 8$ & Down & 0.78482358 & 0.98667434 \\
\hline miR-8067 & -0.0070745 & Down & 0.78450315 & 0.98667434 \\
\hline miR-675I-5p & $-0.00704 \mid 4$ & Down & 0.82126995 & 0.99185328 \\
\hline miR-6748-3p & -0.0069865 & Down & 0.77607548 & 0.985258 \\
\hline miR-374a-3p & -0.006964 & Down & 0.8671137 & $0.998987 / 2$ \\
\hline miR-1247-5p & -0.0069552 & Down & 0.79780852 & 0.98759926 \\
\hline miR-I206 & -0.0069354 & Down & 0.88342507 & $0.998987 \mid 2$ \\
\hline miR-67/6-3p & -0.0069342 & Down & 0.88604018 & $0.998987 \mid 2$ \\
\hline miR-7I53-3p & -0.0068762 & Down & 0.80682369 & 0.99064883 \\
\hline miR-635 & -0.0068419 & Down & 0.87010589 & $0.998987 / 2$ \\
\hline miR-6I6-5p & -0.0067948 & Down & 0.87844038 & 0.99898712 \\
\hline $\operatorname{miR}-548 q$ & -0.0067864 & Down & 0.80815498 & 0.99064883 \\
\hline miR-6832-5p & -0.0067224 & Down & $0.8577830 \mathrm{I}$ & $0.998987 \mid 2$ \\
\hline miR-2682-3p & -0.0066297 & Down & $0.7994 \mid I 31$ & 0.98820926 \\
\hline miR-4I2-5p & -0.00662 & Down & 0.89035774 & 0.99898712 \\
\hline miR-302f & -0.0066155 & Down & $0.88|32| 5 \mid$ & $0.998987 \mid 2$ \\
\hline miR-3064-3p & -0.0065595 & Down & 0.82587428 & 0.99340905 \\
\hline miR-34a-3p & -0.0063862 & Down & $0.87642 \mid 48$ & $0.998987 / 2$ \\
\hline miR-68I0-5p & -0.0063765 & Down & 0.84789718 & 0.99654281 \\
\hline miR-65I2-3p & -0.0063262 & Down & 0.81724812 & 0.99127826 \\
\hline miR-4756-3p & -0.0062289 & Down & 0.86071954 & 0.99898712 \\
\hline miR-6772-3p & -0.0062016 & Down & 0.8176216 & 0.99127826 \\
\hline miR-I I78-3p & -0.0060641 & Down & 0.83603672 & 0.99654281 \\
\hline miR-3I56-3p & -0.0060097 & Down & 0.87659355 & $0.998987 \mid 2$ \\
\hline miR-92a-2-5p & -0.0059985 & Down & 0.89337705 & $0.998987 \mid 2$ \\
\hline miR-3192-5p & -0.0059849 & Down & 0.85140192 & 0.99898712 \\
\hline$m i R-3616-5 p$ & -0.0059161 & Down & 0.87556977 & 0.99898712 \\
\hline$m i R-216 b-5 p$ & -0.0059046 & Down & 0.78988169 & 0.98667434 \\
\hline miR-1273g-5p & -0.0058234 & Down & 0.84875466 & 0.99693761 \\
\hline miR-454-5p & -0.0056392 & Down & 0.87659395 & $0.998987 \mid 2$ \\
\hline miR-604 & -0.0056228 & Down & 0.84115808 & 0.99654281 \\
\hline miR-5003-3p & -0.0055939 & Down & 0.77709499 & 0.985258 \\
\hline miR-4646-5p & -0.0055568 & Down & 0.91343785 & $0.998987 \mid 2$ \\
\hline miR-I I 85-5p & -0.0054925 & Down & 0.8750581 & $0.998987 \mid 2$ \\
\hline miR-6809-3p & -0.0054802 & Down & 0.90618848 & 0.99898712 \\
\hline miR-5695 & $-0.0054 \mid 38$ & Down & 0.84065578 & 0.99654281 \\
\hline miR-1976 & -0.0053957 & Down & $0.8978774 \mid$ & $0.998987 \mid 2$ \\
\hline miR-563 & -0.0053882 & Down & 0.91226822 & $0.998987 / 2$ \\
\hline miR-6734-3p & -0.0053774 & Down & 0.87471748 & $0.998987 / 2$ \\
\hline miR-43I9 & -0.0053747 & Down & 0.86232995 & 0.99898712 \\
\hline miR-342-3p & -0.0052338 & Down & 0.97293249 & 0.99898712 \\
\hline miR-6895-5p & -0.005215 & Down & 0.8608465 & $0.998987 / 2$ \\
\hline miR-4723-5p & -0.0051688 & Down & 0.81224515 & 0.99064883 \\
\hline $\mathrm{miR}-4528$ & -0.0051614 & Down & 0.92980523 & 0.99898712 \\
\hline NC2_000792I5 & -0.0051563 & Down & 0.87570139 & $0.998987 \mid 2$ \\
\hline
\end{tabular}

(Continued) 
Table 9 (Continued).

\begin{tabular}{|c|c|c|c|c|}
\hline miRNAs & Log2FoldChange & Regulation & P-value & P-adj \\
\hline miR-I538 & -0.0050997 & Down & 0.87810329 & 0.99898712 \\
\hline miR-6872-3p & -0.0050274 & Down & 0.88748977 & $0.998987 \mid 2$ \\
\hline $\operatorname{miR}-3668$ & -0.0050174 & Down & 0.90089115 & 0.99898712 \\
\hline miR-4420 & -0.004945 & Down & 0.85326 & $0.998987 / 2$ \\
\hline miR-37la-5p & -0.0049431 & Down & 0.81677945 & 0.99127826 \\
\hline miR-200c-5p & -0.0048674 & Down & 0.86744806 & $0.998987 / 2$ \\
\hline miR-I55-3p & -0.0047673 & Down & 0.92489206 & $0.998987 \mid 2$ \\
\hline miR-6806-5p & -0.0047274 & Down & 0.87183868 & $0.998987 / 2$ \\
\hline miR-920 & -0.0047252 & Down & 0.86831937 & 0.99898712 \\
\hline $\operatorname{miR}-4705$ & -0.0047207 & Down & $0.877|3| 85$ & 0.99898712 \\
\hline miR-9500 & -0.004718 & Down & 0.87680729 & 0.99898712 \\
\hline miR-5704 & -0.0047001 & Down & 0.91499362 & 0.99898712 \\
\hline miR-5694 & -0.0046743 & Down & 0.83419238 & 0.99622199 \\
\hline miR-4685-5p & -0.0046459 & Down & 0.88067889 & $0.998987 / 2$ \\
\hline miR-6504-5p & -0.0043974 & Down & 0.90204635 & $0.998987 / 2$ \\
\hline$m i R-3|5|-5 p$ & $-0.0043 \mid 47$ & Down & 0.8748898 & 0.99898712 \\
\hline miR-6722-5p & -0.0042257 & Down & 0.89460988 & 0.99898712 \\
\hline miR-603 & $-0.004 \mid I 55$ & Down & 0.8667461 & 0.99898712 \\
\hline miR-8052 & -0.0040761 & Down & 0.91117923 & $0.998987 / 2$ \\
\hline miR-4287 & -0.0040522 & Down & 0.89608593 & $0.998987 / 2$ \\
\hline $\operatorname{miR}-4320$ & -0.0040192 & Down & 0.89469173 & $0.998987 / 2$ \\
\hline miR-6838-3p & -0.0039418 & Down & 0.8910569 & $0.998987 / 2$ \\
\hline $\mathrm{miR}-4266$ & -0.0039263 & Down & 0.90249915 & 0.99898712 \\
\hline miR-183-3p & -0.0039116 & Down & 0.90249053 & 0.99898712 \\
\hline miR-770-5p & -0.0038988 & Down & 0.90826697 & 0.99898712 \\
\hline miR-3064-5p & -0.0038875 & Down & $0.89804 \mid 42$ & $0.998987 \mid 2$ \\
\hline miR-634 & -0.0038805 & Down & 0.96723926 & $0.998987 / 2$ \\
\hline miR-660-3p & $-0.00387 / 2$ & Down & 0.9097167 & $0.998987 / 2$ \\
\hline miR-644a & -0.0038296 & Down & 0.9175064 & $0.998987 / 2$ \\
\hline$m i R-5586-3 p$ & $-0.0038 \mid 26$ & Down & 0.86274889 & $0.998987 / 2$ \\
\hline miR-3659 & -0.0037935 & Down & 0.90826795 & $0.998987 \mid 2$ \\
\hline$m i R-512-5 p$ & -0.0037682 & Down & 0.92327364 & 0.99898712 \\
\hline miR-572 & -0.0037248 & Down & 0.91415349 & $0.998987 / 2$ \\
\hline miR-3। 38 & -0.0037108 & Down & 0.91265307 & $0.998987 / 2$ \\
\hline miR-7703 & -0.0037 & Down & 0.9192547 & $0.998987 / 2$ \\
\hline miR-659-3p & -0.0036919 & Down & 0.90801691 & $0.998987 \mid 2$ \\
\hline miR-392I & -0.0036138 & Down & 0.91702854 & 0.99898712 \\
\hline $\operatorname{miR}-23 c$ & $-0.003546 I$ & Down & 0.93580094 & 0.99898712 \\
\hline miR-6769a-5p & -0.003528 & Down & 0.92503826 & $0.998987 / 2$ \\
\hline miR-94I & -0.0035254 & Down & 0.9033374 & $0.998987 / 2$ \\
\hline miR-I 323 & -0.0035254 & Down & 0.9254549 & $0.998987 / 2$ \\
\hline miR-490-3p & -0.0035039 & Down & $0.9059 \mid 256$ & 0.99898712 \\
\hline miR-6504-3p & -0.0034827 & Down & $0.9090686 \mathrm{I}$ & $0.998987 / 2$ \\
\hline miR-I 263 & -0.0034603 & Down & 0.91405859 & $0.998987 / 2$ \\
\hline $\operatorname{miR}-449 a$ & -0.0034566 & Down & 0.90031562 & 0.99898712 \\
\hline miR-3944-5p & -0.003421 & Down & 0.91384492 & 0.99898712 \\
\hline miR-II99-3p & -0.003396 & Down & 0.92236772 & $0.998987 / 2$ \\
\hline miR-3674 & -0.003396 & Down & 0.9206664 & $0.998987 / 2$ \\
\hline$m i R-4659 b-5 p$ & -0.0033209 & Down & 0.93880961 & $0.998987 / 2$ \\
\hline
\end{tabular}

(Continued) 
Table 9 (Continued).

\begin{tabular}{|c|c|c|c|c|}
\hline miRNAs & Log2FoldChange & Regulation & P-value & P-adj \\
\hline miR-3200-3p & -0.0032855 & Down & 0.92266612 & $0.998987 / 2$ \\
\hline miR-450a-I-3p & -0.0032779 & Down & $0.94 \mid 25452$ & $0.998987 \mid 2$ \\
\hline miR-30c-I-3p & $-0.003|64|$ & Down & 0.93461856 & 0.99898712 \\
\hline miR-548w & -0.0031582 & Down & 0.92537786 & 0.99898712 \\
\hline miR-4690-3p & $-0.003|05|$ & Down & 0.92408199 & 0.99898712 \\
\hline miR-6849-5p & -0.0030846 & Down & 0.91722387 & 0.99898712 \\
\hline miR-7II3-3p & -0.0030742 & Down & $0.932277 \mid I$ & 0.99898712 \\
\hline miR-606 & -0.0030314 & Down & 0.91669444 & 0.99898712 \\
\hline miR-455-5p & -0.0030307 & Down & 0.94950521 & 0.99898712 \\
\hline miR-5197-5p & -0.0030231 & Down & 0.96329908 & 0.99898712 \\
\hline miR-6726-5p & -0.0030115 & Down & 0.89240213 & 0.99898712 \\
\hline miR-135b-3p & -0.0029845 & Down & 0.93259063 & 0.99898712 \\
\hline miR-445I & -0.0029258 & Down & 0.90566083 & $0.998987 \mid 2$ \\
\hline miR-5196-3p & -0.0028833 & Down & 0.9410239 & 0.99898712 \\
\hline miR-30a-3p & -0.0028798 & Down & 0.96173026 & 0.99898712 \\
\hline miR-7I59-3p & -0.0028746 & Down & 0.93108389 & 0.99898712 \\
\hline$m i R-144-5 p$ & -0.0028729 & Down & 0.98291237 & 0.99898712 \\
\hline$m i R-204-5 p$ & -0.0027756 & Down & 0.96390565 & 0.99898712 \\
\hline miR-6729-5p & -0.0027536 & Down & 0.9342249 & 0.99898712 \\
\hline miR-524-3p & -0.0027175 & Down & 0.93463627 & $0.998987 \mid 2$ \\
\hline miR-4692 & -0.0027174 & Down & 0.93330804 & $0.998987 \mid 2$ \\
\hline miR-4254 & -0.0027026 & Down & 0.94607389 & $0.998987 / 2$ \\
\hline$m i R-197-3 p$ & $-0.00268 \mid 4$ & Down & 0.97446832 & 0.99898712 \\
\hline miR-6808-5p & -0.0026431 & Down & 0.94489125 & $0.998987 / 2$ \\
\hline miR-6795-5p & -0.0026283 & Down & 0.91449896 & $0.998987 \mid 2$ \\
\hline miR-4324 & -0.00262 & Down & 0.9400151 & $0.998987 \mid 2$ \\
\hline miR-5I3b-3p & -0.0024873 & Down & 0.93432363 & 0.99898712 \\
\hline miR-193b-3p & -0.0024777 & Down & 0.98359496 & 0.99898712 \\
\hline miR-3664-3p & -0.0024542 & Down & 0.92943662 & 0.99898712 \\
\hline miR-6743-5p & -0.0024529 & Down & 0.9027108 & 0.99898712 \\
\hline miR-6507-5p & -0.0024365 & Down & 0.93017877 & 0.99898712 \\
\hline miR-5692b & -0.0024253 & Down & 0.90975261 & 0.99898712 \\
\hline miR-3I58-5p & -0.0024079 & Down & 0.93792342 & 0.99898712 \\
\hline miR-758-5p & -0.002329 & Down & 0.95116671 & 0.99898712 \\
\hline miR-I 283 & -0.0023082 & Down & 0.95634844 & 0.99898712 \\
\hline miR-65I0-3p & $-0.002277 \mid$ & Down & 0.94891395 & 0.99898712 \\
\hline$m i R-3|2|-5 p$ & -0.0022103 & Down & 0.97235279 & $0.998987 / 2$ \\
\hline miR-25-3p & -0.0021921 & Down & 0.97945036 & 0.99898712 \\
\hline miR-368I-3p & -0.0021322 & Down & 0.9550712 & 0.99898712 \\
\hline miR-6887-3p & -0.0020516 & Down & 0.95483442 & 0.99898712 \\
\hline$m i R-10 I-5 p$ & -0.0020024 & Down & 0.96328694 & $0.998987 \mid 2$ \\
\hline miR-6799-5p & -0.0019956 & Down & 0.96608755 & $0.998987 / 2$ \\
\hline miR-219b-3p & $-0.00|964|$ & Down & 0.95601519 & 0.99898712 \\
\hline miR-580-3p & -0.0019317 & Down & 0.94837611 & 0.99898712 \\
\hline miR-6792-3p & -0.0019166 & Down & 0.956652 & 0.99898712 \\
\hline NCl_000002I5 & $-0.001884 \mid$ & Down & 0.96373938 & 0.99898712 \\
\hline miR-68II-5p & -0.0018232 & Down & 0.96188232 & 0.99898712 \\
\hline miR-4704-3p & -0.0017889 & Down & 0.96132284 & $0.998987 \mid 2$ \\
\hline miR-4309 & $-0.001766 \mid$ & Down & 0.95105748 & $0.998987 \mid 2$ \\
\hline
\end{tabular}

(Continued) 
Table 9 (Continued).

\begin{tabular}{|c|c|c|c|c|}
\hline miRNAs & Log2FoldChange & Regulation & P-value & P-adj \\
\hline miR-2392 & -0.0017598 & Down & 0.980127 & 0.99898712 \\
\hline miR-4632-3p & -0.0017532 & Down & 0.94929584 & 0.99898712 \\
\hline miR-2|8-2-3p & -0.0017235 & Down & 0.9674829 & 0.99898712 \\
\hline miR-7II & -0.0016486 & Down & $0.9573 \mid 286$ & 0.99898712 \\
\hline miR-4290 & -0.0016305 & Down & 0.97739464 & $0.998987 \mid 2$ \\
\hline miR-5I89-5p & -0.0016252 & Down & $0.962|508|$ & 0.99898712 \\
\hline miR-500b-5p & -0.001612 & Down & 0.95268854 & 0.99898712 \\
\hline miR-3648 & -0.0014824 & Down & 0.96589432 & 0.99898712 \\
\hline $\operatorname{miR}-4674$ & -0.0014518 & Down & 0.94880466 & 0.99898712 \\
\hline miR-I 208 & -0.0013905 & Down & 0.96583714 & 0.99898712 \\
\hline miR-475I & -0.0013897 & Down & 0.95352909 & 0.99898712 \\
\hline$m i R-433-3 p$ & -0.0013693 & Down & 0.97819644 & 0.99898712 \\
\hline miR-4308 & -0.0013122 & Down & 0.95611105 & 0.99898712 \\
\hline$m i R-548 j-5 p$ & -0.0012819 & Down & 0.97553329 & 0.99898712 \\
\hline miR-8068 & -0.0012147 & Down & 0.95925337 & 0.99898712 \\
\hline miR-6759-5p & -0.0011223 & Down & 0.96731 & 0.99898712 \\
\hline $\operatorname{miR}-67 \mid 5 b-3 p$ & -0.0011213 & Down & 0.97036889 & 0.99898712 \\
\hline miR-548an & -0.0011134 & Down & 0.96318494 & 0.99898712 \\
\hline miR-67I5b-5p & -0.0010821 & Down & 0.9703719 & 0.99898712 \\
\hline$m i R-510-5 p$ & -0.0010532 & Down & $0.97075 \mid 47$ & $0.998987 \mid 2$ \\
\hline miR-4694-5p & -0.0010357 & Down & 0.98024922 & $0.998987 \mid 2$ \\
\hline miR-68I7-5p & -0.0010043 & Down & 0.96991745 & 0.99898712 \\
\hline miR-3606-3p & -0.0009929 & Down & 0.98932447 & 0.99904195 \\
\hline $\operatorname{miR}-3609$ & -0.0009853 & Down & 0.97323962 & 0.99898712 \\
\hline$m i R-5 I 8 b$ & -0.000889 & Down & 0.98279757 & 0.99898712 \\
\hline miR-124-3p & -0.0008157 & Down & 0.97080446 & 0.99898712 \\
\hline miR-I 204 & -0.0007893 & Down & $0.9856 \mid 309$ & 0.99898712 \\
\hline miR-I255b-5p & -0.0007106 & Down & 0.98083044 & 0.99898712 \\
\hline miR-6837-5p & -0.0006794 & Down & 0.98080458 & 0.99898712 \\
\hline miR-507 & -0.0006167 & Down & 0.98680757 & 0.99904195 \\
\hline miR-96-3p & -0.0005904 & Down & 0.98939967 & 0.99904195 \\
\hline miR-3 I86-5p & -0.0005172 & Down & 0.98303093 & 0.99898712 \\
\hline miR-370-5p & -0.0005016 & Down & $0.9854034 I$ & 0.99898712 \\
\hline miR-4273 & -0.00045 & Down & 0.99087488 & 0.99904195 \\
\hline miR-6798-3p & -0.0004329 & Down & 0.99242048 & 0.99904195 \\
\hline miR-3I 22 & -0.0004157 & Down & 0.98547863 & 0.99898712 \\
\hline miR-39II & -0.0003118 & Down & 0.99522027 & 0.99904195 \\
\hline miR-37lb-3p & -0.0003055 & Down & 0.99364604 & 0.99904195 \\
\hline miR-6868-5p & $-0.00028 I I$ & Down & 0.99205446 & 0.99904195 \\
\hline miR-299-3p & -0.0002789 & Down & 0.99354178 & 0.99904195 \\
\hline miR-302c-5p & -0.0002069 & Down & 0.99238835 & 0.99904195 \\
\hline miR-6505-3p & -0.0002018 & Down & 0.99670956 & 0.99904195 \\
\hline miR-548bb-3p & $4.80 \times 10^{-5}$ & $U_{p}$ & 0.99929046 & 1 \\
\hline$m i R-627-3 p$ & $4.86 \times 10^{-5}$ & $U_{p}$ & 0.99946876 & I \\
\hline miR-67/8-5p & 0.00012601 & $U_{p}$ & 0.99652509 & 0.99904195 \\
\hline miR-6790-5p & 0.00014766 & Up & 0.9958867 & 0.99904195 \\
\hline miR-4666a-3p & 0.00017412 & $U_{p}$ & 0.99603995 & 0.99904195 \\
\hline $\mathrm{miR}-302 \mathrm{e}$ & 0.00019522 & Up & 0.99636872 & 0.99904195 \\
\hline miR-6787-5p & 0.00026324 & Up & $0.993 \mid 2444$ & 0.99904195 \\
\hline
\end{tabular}

(Continued) 
Table 9 (Continued).

\begin{tabular}{|c|c|c|c|c|}
\hline miRNAs & Log2FoldChange & Regulation & P-value & P-adj \\
\hline miR-I47b & 0.00029406 & Up & 0.99417609 & 0.99904195 \\
\hline miR-4740-5p & 0.00029998 & Up & 0.99473955 & 0.99904195 \\
\hline miR-4800-3p & 0.0003067 & Up & 0.99309816 & 0.99904195 \\
\hline miR-3683 & 0.00036807 & Up & $0.9909276 \mathrm{I}$ & 0.99904195 \\
\hline miR-3689a-5p & 0.00038906 & Up & 0.98808154 & 0.99904195 \\
\hline miR-6827-3p & 0.0004124 & Up & 0.99192316 & 0.99904195 \\
\hline miR-5089-5p & 0.00043586 & Up & 0.99014203 & 0.99904195 \\
\hline miR-3I46 & 0.00045422 & Up & 0.98491092 & 0.99898712 \\
\hline miR-479I & 0.00046421 & Up & 0.98577094 & $0.998987 / 2$ \\
\hline miR-4670-3p & 0.00049717 & Up & 0.98246602 & 0.99898712 \\
\hline miR-6I 29 & 0.00052485 & Up & 0.98770083 & 0.99904195 \\
\hline miR-4798-3p & 0.00052915 & Up & 0.98085346 & $0.998987 \mid 2$ \\
\hline miR-7845-5p & $0.00054 \mid 49$ & $U_{p}$ & 0.98878954 & 0.99904195 \\
\hline miR-587 & 0.00056284 & Up & 0.98476648 & $0.998987 / 2$ \\
\hline miR-4706 & 0.00057658 & Up & 0.98417593 & 0.99898712 \\
\hline miR-3650 & 0.00058852 & Up & 0.98065605 & $0.998987 / 2$ \\
\hline miR-4537 & 0.00060113 & Up & 0.98049055 & $0.998987 \mid 2$ \\
\hline miR-6735-3p & 0.00062976 & Up & 0.98562082 & 0.99898712 \\
\hline miR-4749-5p & 0.00064826 & Up & 0.98398478 & $0.998987 / 2$ \\
\hline miR-3। 85 & 0.00065819 & Up & 0.97570375 & $0.998987 \mid 2$ \\
\hline miR-89la-3p & 0.00066649 & Up & $0.984707 \mid 3$ & $0.998987 / 2$ \\
\hline miR-3529-5p & 0.00069812 & Up & 0.98705831 & 0.99904195 \\
\hline miR-520f-3p & 0.00079577 & $U_{p}$ & 0.98055025 & $0.998987 / 2$ \\
\hline miR-5703 & 0.00079607 & $U_{p}$ & 0.97556576 & $0.998987 / 2$ \\
\hline miR-4535 & $0.00083 \mid 55$ & Up & 0.98315972 & $0.998987 / 2$ \\
\hline$m i R-4793-3 p$ & 0.00087755 & Up & 0.98068497 & $0.998987 \mid 2$ \\
\hline miR-3649 & 0.00090916 & Up & 0.96843892 & $0.998987 \mid 2$ \\
\hline miR-3655 & 0.0009646 & Up & 0.97337242 & $0.998987 / 2$ \\
\hline miR-6806-3p & 0.00100569 & Up & 0.98161655 & $0.998987 / 2$ \\
\hline miR-I304-5p & 0.00104513 & Up & $0.96964 \mid 37$ & $0.998987 / 2$ \\
\hline miR-3। 48 & 0.001135 & $U_{p}$ & 0.96629547 & 0.99898712 \\
\hline miR-I298-3p & 0.00116617 & $U_{p}$ & 0.96906897 & $0.998987 / 2$ \\
\hline miR-378a-5p & 0.00119067 & Up & 0.99508311 & 0.99904195 \\
\hline miR-7I6I-3p & 0.00122334 & Up & 0.97003457 & $0.998987 \mid 2$ \\
\hline miR-23a-3p & 0.00125302 & Up & 0.99132986 & 0.99904195 \\
\hline miR-6849-3p & 0.00127333 & Up & 0.97699099 & $0.998987 \mid 2$ \\
\hline miR-3|9|-5p & 0.00129186 & $U_{p}$ & $0.96|2572|$ & $0.998987 \mid 2$ \\
\hline$m i R-4699-5 p$ & 0.00129705 & Up & 0.97103377 & $0.998987 \mid 2$ \\
\hline miR-3|26-3p & 0.00139265 & Up & 0.95926798 & $0.998987 / 2$ \\
\hline miR-3|40-5p & 0.00140044 & Up & 0.9636057 & $0.998987 / 2$ \\
\hline$m i R-6723-5 p$ & 0.00142556 & Up & $0.97534 \mid I 3$ & $0.998987 \mid 2$ \\
\hline miR-573 & $0.0014267 \mid$ & Up & 0.95512489 & $0.998987 / 2$ \\
\hline$m i R-4733-5 p$ & 0.00143716 & Up & 0.97172874 & $0.998987 / 2$ \\
\hline miR-92b-3p & 0.00146746 & Up & 0.97672286 & $0.998987 / 2$ \\
\hline miR-II80-5p & 0.00147286 & Up & 0.95840719 & $0.998987 / 2$ \\
\hline$m i R-4768-3 p$ & 0.00148174 & Up & 0.96684936 & $0.998987 / 2$ \\
\hline miR-6804-3p & 0.00150994 & Up & $0.963|259|$ & $0.998987 / 2$ \\
\hline$m i R-50 I 0-5 p$ & 0.00150997 & Up & 0.95909548 & $0.998987 / 2$ \\
\hline miR-5007-3p & 0.00153164 & $U_{p}$ & 0.98261968 & $0.998987 / 2$ \\
\hline
\end{tabular}

(Continued) 
Table 9 (Continued).

\begin{tabular}{|c|c|c|c|c|}
\hline miRNAs & Log2FoldChange & Regulation & P-value & P-adj \\
\hline miR-7I53-5p & 0.00153251 & $U_{p}$ & 0.95803798 & 0.99898712 \\
\hline miR-4789-5p & 0.00153826 & Up & 0.97000256 & $0.998987 \mid 2$ \\
\hline miR-149-5p & 0.00169817 & Up & 0.95691905 & 0.99898712 \\
\hline miR-8077 & 0.00173739 & Up & $0.9622858 I$ & $0.998987 / 2$ \\
\hline miR-127-5p & 0.00173908 & Up & 0.95183069 & $0.998987 / 2$ \\
\hline miR-450I & 0.00175705 & Up & 0.95082433 & $0.998987 / 2$ \\
\hline$m i R-376 a-5 p$ & 0.00176507 & Up & 0.96843308 & $0.998987 \mid 2$ \\
\hline miR-4279 & 0.00178895 & Up & 0.9671217 & $0.998987 / 2$ \\
\hline miR-I 282 & 0.00180239 & Up & 0.9642268 & $0.998987 / 2$ \\
\hline$m i R-5583-5 p$ & 0.00182263 & $U_{p}$ & 0.97743092 & 0.99898712 \\
\hline miR-4437 & 0.0018404 & Up & 0.95314944 & $0.998987 / 2$ \\
\hline miR-443I & 0.00185753 & Up & 0.94002562 & 0.99898712 \\
\hline miR-548ap-3p & 0.00191567 & Up & 0.96952875 & 0.99898712 \\
\hline miR-30b-3p & 0.00192048 & Up & 0.96868055 & $0.998987 / 2$ \\
\hline miR-588 & 0.00194739 & Up & 0.95288558 & $0.998987 / 2$ \\
\hline miR-1228-5p & 0.00201888 & $U_{p}$ & 0.92703988 & 0.99898712 \\
\hline miR-5687 & 0.0020738 & $U_{p}$ & 0.96196251 & 0.99898712 \\
\hline$m i R-6816-3 p$ & 0.00215482 & $U_{p}$ & 0.95631146 & 0.99898712 \\
\hline miR-519c-3p & 0.00215986 & $U_{p}$ & 0.95475733 & $0.998987 / 2$ \\
\hline miR-4703-3p & 0.00216083 & $U_{p}$ & 0.96161646 & $0.998987 / 2$ \\
\hline miR-4655-3p & 0.00219685 & $U_{p}$ & 0.94507478 & $0.998987 / 2$ \\
\hline miR-I295a & 0.00219999 & $U_{p}$ & 0.93784315 & $0.998987 / 2$ \\
\hline miR-3940-3p & $0.0022904 I$ & $U_{p}$ & 0.96273026 & 0.99898712 \\
\hline $\operatorname{miR}-4719$ & 0.00229203 & $U_{p}$ & 0.96654653 & 0.99898712 \\
\hline$m i R-4677-5 p$ & 0.0022962 & $U_{p}$ & 0.94525282 & 0.99898712 \\
\hline miR-6847-3p & 0.00230232 & $U_{p}$ & 0.96282302 & $0.998987 \mid 2$ \\
\hline miR-4684-5p & 0.00237006 & Up & 0.94302918 & $0.998987 / 2$ \\
\hline$m i R-4769-5 p$ & 0.00238533 & Up & 0.93039316 & $0.998987 / 2$ \\
\hline miR-4289 & 0.00239412 & Up & 0.95728448 & $0.998987 / 2$ \\
\hline miR-300 & 0.00239907 & $U_{p}$ & 0.94468572 & $0.998987 / 2$ \\
\hline$m i R-6829-3 p$ & 0.00243816 & $U_{p}$ & $0.9296 \mid 757$ & $0.998987 \mid 2$ \\
\hline$m i R-4432$ & $0.0024506 I$ & Up & 0.90864201 & 0.99898712 \\
\hline miR-4524b-3p & 0.002466 & Up & 0.94444986 & $0.998987 / 2$ \\
\hline$m i R-552-5 p$ & 0.00249528 & Up & 0.94630486 & $0.998987 / 2$ \\
\hline miR-1247-3p & 0.00258503 & Up & 0.9230124 & $0.998987 / 2$ \\
\hline miR-44I7 & 0.0027244 & Up & 0.94819632 & $0.998987 \mid 2$ \\
\hline miR-195-3p & 0.00280151 & Up & 0.94134865 & $0.998987 / 2$ \\
\hline$m i R-4763-5 p$ & 0.00283221 & Up & $0.94567 \mid 28$ & 0.99898712 \\
\hline miR-559 & 0.00283475 & Up & 0.93246615 & $0.998987 / 2$ \\
\hline miR-1288-5p & 0.00287294 & Up & 0.91069106 & $0.998987 / 2$ \\
\hline miR-3I 28 & 0.00290191 & $U_{p}$ & 0.91664185 & $0.998987 / 2$ \\
\hline miR-335-3p & 0.0030629 & Up & 0.96743191 & 0.99898712 \\
\hline miR-92a-3p & 0.00309934 & Up & 0.966666665 & $0.998987 / 2$ \\
\hline miR-328-5p & $0.003|875|$ & Up & 0.91996491 & $0.998987 / 2$ \\
\hline $\mathrm{miR}-8058$ & 0.00322494 & $U_{p}$ & 0.93576164 & 0.99898712 \\
\hline miR-3654 & 0.00324715 & Up & 0.9154113 & $0.998987 / 2$ \\
\hline miR-330-5p & 0.00325701 & $U_{p}$ & 0.94317225 & $0.998987 / 2$ \\
\hline miR-5089-3p & 0.00328345 & Up & 0.92178001 & $0.998987 / 2$ \\
\hline miR-6830-3p & 0.0032993 & $U_{p}$ & 0.92150396 & $0.998987 / 2$ \\
\hline
\end{tabular}

(Continued) 
Table 9 (Continued).

\begin{tabular}{|c|c|c|c|c|}
\hline miRNAs & Log2FoldChange & Regulation & P-value & P-adj \\
\hline$m i R-4752$ & 0.00330367 & Up & 0.93028464 & 0.99898712 \\
\hline$m i R-33 I-5 p$ & 0.00331525 & Up & 0.92813569 & 0.99898712 \\
\hline $\mathrm{miR}-663 \mathrm{~b}$ & 0.00331973 & Up & 0.91301131 & 0.99898712 \\
\hline miR-4764-3p & 0.00336323 & Up & 0.93474718 & 0.99898712 \\
\hline let-7b-3p & 0.00337109 & Up & 0.95072657 & 0.99898712 \\
\hline miR-548aw & 0.00338056 & $U_{p}$ & 0.93884878 & 0.99898712 \\
\hline$m i R-|27|-3 p$ & 0.0034496 & $U_{p}$ & 0.89829719 & $0.998987 \mid 2$ \\
\hline miR-5590-3p & 0.00347784 & Up & 0.92848311 & 0.99898712 \\
\hline miR-65। la-5p & 0.00354836 & Up & 0.89798915 & 0.99898712 \\
\hline miR-9-3p & 0.00359347 & Up & 0.92054605 & 0.99898712 \\
\hline miR-5II-5p & 0.00361469 & $U_{p}$ & 0.9155092 & 0.99898712 \\
\hline$m i R-516 a-3 p$ & 0.00364134 & $U_{p}$ & 0.91537755 & 0.99898712 \\
\hline miR-5I86 & 0.00366302 & $U_{p}$ & 0.8725406 & 0.99898712 \\
\hline miR-885-3p & 0.00369562 & $U_{p}$ & 0.85885838 & 0.99898712 \\
\hline miR-4456 & 0.00380003 & $U_{p}$ & 0.9123011 & 0.99898712 \\
\hline miR-8086 & 0.00380099 & $U_{p}$ & 0.88474931 & 0.99898712 \\
\hline$m i R-6816-5 p$ & 0.0038261 & $U_{p}$ & 0.92075975 & 0.99898712 \\
\hline $\operatorname{miR}-4274$ & 0.00389749 & Up & 0.9244925 & 0.99898712 \\
\hline miR-3909 & 0.003979 & $U_{p}$ & $0.9103956 \mid$ & $0.998987 / 2$ \\
\hline miR-3978 & 0.00405802 & $U_{p}$ & 0.91465819 & $0.998987 \mid 2$ \\
\hline$m i R-6743-3 p$ & 0.00407426 & $U_{p}$ & $0.8897 \mid 427$ & $0.998987 \mid 2$ \\
\hline miR-4732-5p & 0.00428378 & $U_{p}$ & 0.86998959 & $0.998987 \mid 2$ \\
\hline miR-610 & 0.00430615 & $U_{p}$ & $0.878 \mid 7427$ & $0.998987 / 2$ \\
\hline miR-3157-5p & 0.0043107 & $U_{p}$ & 0.87760033 & $0.998987 \mid 2$ \\
\hline$m i R-16-5 p$ & $0.00434 \mid 27$ & $U_{p}$ & 0.93567182 & $0.998987 \mid 2$ \\
\hline miR-379-3p & $0.0044 \mid 424$ & $U_{p}$ & 0.90423587 & $0.998987 \mid 2$ \\
\hline miR-6796-5p & 0.00443933 & $U_{p}$ & $0.87 \mid 39356$ & $0.998987 \mid 2$ \\
\hline miR-4450 & 0.00444525 & $U_{p}$ & 0.91178 & $0.998987 \mid 2$ \\
\hline miR-8059 & 0.00447583 & $U_{p}$ & $0.9126362 \mid$ & 0.99898712 \\
\hline$m i R-6746-3 p$ & 0.00448799 & $U_{p}$ & 0.90899954 & 0.99898712 \\
\hline miR-4255 & 0.00458999 & Up & 0.84328656 & $0.9965428 \mid$ \\
\hline miR-II 83 & 0.0047103 & $U_{p}$ & 0.856079 & 0.99898712 \\
\hline miR-760 & 0.00477938 & Up & 0.92370615 & 0.99898712 \\
\hline miR-4495 & 0.00479005 & $U_{p}$ & 0.86428986 & 0.99898712 \\
\hline miR-7I52-5p & $0.0048 \mid 184$ & $U_{p}$ & 0.93769352 & $0.998987 \mid 2$ \\
\hline miR-8064 & 0.00496846 & $U_{p}$ & $0.8845986 I$ & $0.998987 \mid 2$ \\
\hline$m i R-7-5 p$ & 0.00499621 & $U_{p}$ & 0.96784354 & $0.998987 \mid 2$ \\
\hline$m i R-6748-5 p$ & 0.00501023 & $U_{p}$ & 0.84754757 & $0.9965428 I$ \\
\hline miR-4462 & 0.00504316 & $U_{p}$ & 0.9290548 & $0.998987 / 2$ \\
\hline miR-1908-5p & 0.00506377 & Up & 0.84310963 & $0.9965428 \mathrm{I}$ \\
\hline miR-4306 & 0.00507078 & $U_{p}$ & 0.88556954 & $0.998987 \mid 2$ \\
\hline miR-2I7 & 0.00510601 & $U_{p}$ & 0.84588598 & $0.9965428 \mathrm{I}$ \\
\hline$m i R-4640-5 p$ & $0.005|3| 23$ & $U_{p}$ & $0.8745 \mid 458$ & $0.998987 \mid 2$ \\
\hline miR-3928-3p & $0.005 \mid 8066$ & $U_{p}$ & 0.83609674 & $0.9965428 \mathrm{I}$ \\
\hline miR-520g-3p & 0.00520766 & $U_{p}$ & 0.90908556 & $0.998987 \mid 2$ \\
\hline miR-6890-5p & 0.00522115 & Up & 0.85094437 & $0.998987 \mid 2$ \\
\hline miR-I288-3p & 0.00522151 & $U_{p}$ & 0.93793162 & 0.99898712 \\
\hline miR-548aq-5p & 0.00523516 & $U_{p}$ & 0.88953513 & $0.998987 / 2$ \\
\hline miR-6078 & 0.00525155 & $U_{p}$ & 0.87577069 & $0.998987 / 2$ \\
\hline
\end{tabular}

(Continued) 
Table 9 (Continued).

\begin{tabular}{|c|c|c|c|c|}
\hline miRNAs & Log2FoldChange & Regulation & P-value & P-adj \\
\hline miR-39I5 & 0.00526613 & $U_{p}$ & 0.83166795 & 0.99505896 \\
\hline miR-67/9-3p & 0.00527162 & Up & 0.88460055 & 0.99898712 \\
\hline miR-4436b-3p & 0.00527661 & Up & 0.84266097 & 0.99654281 \\
\hline miR-4283 & 0.00527869 & Up & 0.8450852 & $0.9965428 \mathrm{I}$ \\
\hline miR-593-5p & 0.00531576 & Up & $0.847890 \mid 4$ & $0.9965428 I$ \\
\hline miR-6839-3p & 0.00532191 & Up & 0.87262952 & 0.99898712 \\
\hline miR-3927-5p & 0.00548928 & Up & 0.86784757 & 0.99898712 \\
\hline miR-4643 & 0.00549469 & Up & 0.89871642 & $0.998987 / 2$ \\
\hline miR-626 & 0.0055202 & Up & 0.82249207 & 0.99252298 \\
\hline miR-6070 & 0.00553692 & Up & 0.86897821 & 0.99898712 \\
\hline miR-6074 & 0.00554496 & Up & 0.83968303 & $0.9965428 I$ \\
\hline miR-548ak & 0.00559526 & Up & 0.86340327 & 0.99898712 \\
\hline miR-26a-2-3p & 0.00561578 & Up & 0.93206718 & 0.99898712 \\
\hline miR-6758-5p & $0.0056784 \mid$ & Up & 0.81085101 & 0.99064883 \\
\hline miR-37I3 & 0.00577598 & Up & 0.77736592 & 0.985258 \\
\hline$m i R-2116-3 p$ & 0.00580625 & Up & 0.90984233 & 0.99898712 \\
\hline miR-785I-3p & $0.00588 \mid 84$ & Up & $0.844 \mid 5095$ & 0.99654281 \\
\hline miR-4803 & 0.00597366 & Up & 0.88088685 & $0.998987 \mid 2$ \\
\hline$m i R-5 \mid 4 a-3 p$ & 0.00604114 & Up & 0.86688525 & $0.998987 / 2$ \\
\hline miR-1972 & 0.00604884 & Up & 0.91631474 & 0.99898712 \\
\hline miR-875-3p & $0.00605 \mid 47$ & Up & 0.81226805 & 0.99064883 \\
\hline miR-302d-5p & $0.0060967 \mid$ & Up & 0.82642356 & 0.99340905 \\
\hline miR-679I-3p & 0.00611437 & Up & 0.82015659 & 0.99144047 \\
\hline miR-23a-5p & 0.00611826 & $U_{p}$ & 0.84035989 & 0.99654281 \\
\hline miR-4690-5p & $0.0062734 I$ & Up & 0.88491742 & 0.99898712 \\
\hline miR-685I-5p & $0.0064 \mid 48$ & Up & 0.91852166 & $0.998987 / 2$ \\
\hline miR-548x-3p & $0.00647 \mid 26$ & Up & 0.78579776 & 0.98667434 \\
\hline miR-4263 & 0.00647493 & Up & 0.82323114 & 0.99252298 \\
\hline miR-30c-2-3p & 0.00658433 & Up & 0.78325746 & 0.98667434 \\
\hline miR-4253 & 0.00662912 & $U_{p}$ & 0.86666295 & $0.998987 / 2$ \\
\hline miR-8085 & 0.0066686 & Up & 0.80768958 & 0.99064883 \\
\hline miR-7I6I-5p & 0.00667439 & Up & 0.83283078 & 0.9955233 \\
\hline miR-6763-3p & 0.00667776 & Up & $0.8786225 I$ & $0.998987 / 2$ \\
\hline miR-3 I 27-3p & 0.00671663 & Up & 0.8253678 & 0.99340905 \\
\hline miR-II 79 & 0.00675695 & Up & 0.8391941 & $0.9965428 I$ \\
\hline miR-4469 & 0.00679696 & Up & 0.79739667 & 0.98759926 \\
\hline miR-4678 & 0.00682196 & Up & $0.84 \mid 56448$ & 0.99654281 \\
\hline miR-6843-3p & 0.00693783 & Up & 0.81870342 & 0.99127826 \\
\hline miR-5590-5p & 0.00699154 & Up & $0.885 \mid 9385$ & $0.998987 / 2$ \\
\hline miR-367-5p & 0.00701992 & Up & $0.762 \mid 473$ & $0.98 \mid 40055$ \\
\hline miR-3657 & $0.00705 \mid 38$ & Up & $0.8678 \mid 429$ & 0.99898712 \\
\hline miR-6500-5p & 0.00709102 & Up & 0.88389959 & 0.99898712 \\
\hline miR-7109-3p & 0.00715258 & Up & 0.86817229 & $0.998987 / 2$ \\
\hline miR-76I & $0.00725|4|$ & Up & 0.80469338 & 0.99064883 \\
\hline miR-3685 & 0.00726365 & Up & 0.79280404 & 0.98676309 \\
\hline miR-47I4-3p & 0.00729509 & Up & 0.85892014 & $0.998987 / 2$ \\
\hline$m i R-6866-5 p$ & 0.00730567 & Up & $0.8462 I 505$ & 0.99654281 \\
\hline miR-4526 & 0.00733506 & $U_{p}$ & 0.76375104 & $0.98 \mid 40055$ \\
\hline miR-4436b-5p & 0.00741513 & Up & 0.88151895 & $0.998987 \mid 2$ \\
\hline
\end{tabular}

(Continued) 
Table 9 (Continued).

\begin{tabular}{|c|c|c|c|c|}
\hline miRNAs & Log2FoldChange & Regulation & P-value & P-adj \\
\hline miR-3I53 & 0.0074168 & Up & $0.7694728 \mathrm{I}$ & 0.98237037 \\
\hline miR-4772-3p & $0.00754 I I$ & Up & 0.81766753 & 0.99127826 \\
\hline miR-3663-5p & 0.00762725 & Up & 0.80974872 & 0.99064883 \\
\hline miR-3065-5p & 0.00770806 & Up & 0.73065485 & 0.97193735 \\
\hline miR-3|4I & 0.00776054 & Up & 0.90607938 & 0.99898712 \\
\hline miR-I292-3p & 0.00776917 & Up & 0.81851928 & 0.99127826 \\
\hline$m i R-4756-5 p$ & 0.00780377 & Up & 0.79286606 & 0.98676309 \\
\hline miR-7976 & $0.0078267 \mid$ & Up & 0.8670542 & 0.99898712 \\
\hline miR-3|40-3p & 0.0078855 & Up & 0.79720547 & 0.98759926 \\
\hline miR-444I & 0.00799964 & Up & 0.70676444 & 0.96360966 \\
\hline$m i R-2 I I-3 p$ & 0.00803022 & Up & 0.87470817 & $0.998987 \mid 2$ \\
\hline miR-3I 74 & $0.0081048 \mid$ & Up & 0.75961032 & 0.98140055 \\
\hline miR-6720-5p & 0.00818865 & $U_{p}$ & 0.84626862 & 0.99654281 \\
\hline miR-4304 & 0.0082361 & Up & 0.79025258 & 0.98667434 \\
\hline miR-I 264 & $0.0086 \mid 208$ & Up & 0.8411249 & $0.9965428 \mathrm{I}$ \\
\hline miR-39|2-3p & $0.008638 \mid 4$ & Up & 0.87433044 & 0.99898712 \\
\hline miR-4506 & 0.00870046 & Up & 0.90739398 & $0.998987 \mid 2$ \\
\hline miR-I 284 & 0.008722 & Up & 0.79085029 & 0.98667434 \\
\hline miR-568Ib & $0.008764 I I$ & Up & $0.8584|3|$ & 0.99898712 \\
\hline miR-548aa & 0.00890056 & Up & 0.84798854 & 0.99654281 \\
\hline miR-4269 & 0.00892899 & Up & 0.81451596 & 0.99122915 \\
\hline miR-7I58-5p & 0.00894845 & Up & 0.86729561 & $0.998987 / 2$ \\
\hline$m i R-452-5 p$ & 0.00895862 & $U_{p}$ & 0.85771099 & 0.99898712 \\
\hline miR-4490 & 0.00898673 & $U_{p}$ & 0.73250027 & 0.9725372 \\
\hline$m i R-656-5 p$ & 0.00904604 & Up & 0.74755094 & 0.97721563 \\
\hline miR-6506-5p & 0.00904757 & Up & 0.67559786 & 0.95648223 \\
\hline$m i R-4755-5 p$ & 0.0090802 & Up & 0.76114498 & 0.98140055 \\
\hline miR-I273a & 0.00909625 & Up & 0.70701626 & 0.96360966 \\
\hline miR-6878-5p & 0.00916874 & Up & 0.83254408 & 0.9955233 \\
\hline$m i R-6840-5 p$ & 0.00917447 & $U_{p}$ & $0.78853 \mid 45$ & 0.98667434 \\
\hline miR-888-5p & 0.00930898 & $U_{p}$ & 0.6728992 & 0.95648223 \\
\hline miR-4494 & 0.00934109 & Up & 0.65694105 & 0.95321673 \\
\hline miR-3689b-3p & 0.00939617 & Up & 0.78293583 & 0.98667434 \\
\hline miR-6804-5p & 0.00939724 & Up & 0.65264057 & 0.95321673 \\
\hline miR-4698 & 0.00943298 & Up & $0.84|4294|$ & 0.99654281 \\
\hline miR-3I 34 & 0.00954703 & Up & 0.73284844 & 0.9725372 \\
\hline$m i R-6880-5 p$ & 0.00956249 & $U_{p}$ & 0.84058856 & 0.99654281 \\
\hline miR-6764-3p & 0.00958155 & Up & 0.7778236 & 0.985258 \\
\hline miR-6840-3p & 0.00960503 & Up & 0.80087863 & 0.98907164 \\
\hline miR-596 & 0.0096344 & Up & 0.6972447 & 0.96239183 \\
\hline$m i R-6823-3 p$ & 0.0096698 & Up & 0.83033428 & 0.99448905 \\
\hline miR-3200-5p & 0.00979753 & Up & 0.82584554 & 0.99340905 \\
\hline$m i R-6828-3 p$ & 0.00989754 & Up & 0.76984199 & 0.98237037 \\
\hline$m i R-4783-3 p$ & 0.00995837 & Up & 0.73689972 & 0.97354829 \\
\hline miR-4670-5p & 0.01004604 & Up & 0.76449957 & 0.98140055 \\
\hline miR-136-3p & 0.01006505 & $U_{p}$ & 0.85730812 & $0.998987 / 2$ \\
\hline miR-365a-5p & 0.01010965 & Up & 0.70048463 & 0.96268515 \\
\hline miR-4748 & 0.01013589 & Up & 0.79150405 & 0.98667434 \\
\hline miR-4742-3p & 0.0101833 & $U_{p}$ & 0.77805094 & 0.985258 \\
\hline
\end{tabular}

(Continued) 
Table 9 (Continued).

\begin{tabular}{|c|c|c|c|c|}
\hline miRNAs & Log2FoldChange & Regulation & P-value & P-adj \\
\hline miR-4520-5p & 0.0102179 & $U_{p}$ & 0.8304177 & 0.99448905 \\
\hline miR-II $80-3 p$ & 0.01021945 & Up & 0.66003638 & 0.95397723 \\
\hline miR-369l-5p & 0.01022264 & Up & 0.72226246 & 0.96627512 \\
\hline$m i R-268 I-5 p$ & 0.01028915 & Up & 0.71152528 & 0.96505269 \\
\hline miR-203a-5p & 0.01029073 & Up & 0.74476166 & 0.97605174 \\
\hline miR-4536-5p & 0.01032611 & Up & $0.798 \mid 4929$ & 0.98759926 \\
\hline miR-548aj-5p & 0.01034554 & Up & 0.76659882 & 0.98213308 \\
\hline miR-3I50a-3p & 0.01034608 & Up & $0.72387 \mid 37$ & 0.96691758 \\
\hline miR-4433a-3p & 0.01036148 & Up & 0.83417748 & 0.99622199 \\
\hline miR-450a-5p & 0.01036798 & Up & 0.93799632 & 0.99898712 \\
\hline miR-5096 & 0.01038371 & Up & 0.71906775 & 0.96505269 \\
\hline miR-4762-5p & 0.01039264 & Up & 0.77610745 & 0.985258 \\
\hline miR-6809-5p & 0.01040392 & Up & 0.79202691 & 0.98667434 \\
\hline miR-3682-5p & 0.01042188 & Up & 0.81098701 & 0.99064883 \\
\hline miR-I295b-3p & 0.01043015 & Up & 0.71909568 & 0.96505269 \\
\hline miR-4444 & 0.01045387 & Up & 0.7631112 & $0.98 \mid 40055$ \\
\hline miR-888-3p & 0.01047353 & Up & 0.76818709 & 0.98237037 \\
\hline miR-302d-3p & 0.01049923 & Up & 0.80356561 & 0.99063213 \\
\hline miR-4520-3p & 0.01052429 & Up & 0.61164525 & 0.94386479 \\
\hline miR-365b-5p & 0.01054114 & Up & 0.79167801 & 0.98667434 \\
\hline miR-490-5p & 0.01063911 & Up & 0.69008095 & 0.96036263 \\
\hline miR-4796-3p & 0.01068572 & Up & $0.695 \mid 2286$ & 0.96185982 \\
\hline miR-6883-5p & 0.01079249 & Up & 0.65690962 & 0.95321673 \\
\hline miR-6766-5p & 0.01097063 & $U_{p}$ & 0.73980817 & 0.97402999 \\
\hline miR-5707 & 0.01098079 & Up & 0.7204253 & 0.96558066 \\
\hline miR-3/87-5p & 0.01105233 & Up & 0.61637057 & 0.94741169 \\
\hline miR-7846-3p & 0.0110547 & Up & 0.76558774 & $0.98|4768|$ \\
\hline miR-6777-5p & 0.01111177 & Up & 0.65793259 & 0.95321673 \\
\hline miR-4258 & 0.01123609 & Up & $0.8400 \mid 222$ & 0.99654281 \\
\hline miR-4797-5p & 0.01124647 & $U_{p}$ & 0.68924997 & 0.96036263 \\
\hline miR-18Ia-5p & 0.01129006 & Up & 0.93201014 & $0.998987 / 2$ \\
\hline miR-4734 & 0.0113266 & Up & 0.68320782 & 0.95853044 \\
\hline miR-5682 & 0.01146077 & Up & 0.54685586 & 0.92261663 \\
\hline miR-5589-5p & 0.01146606 & Up & 0.68330492 & 0.95853044 \\
\hline miR-1296-3p & 0.01148509 & Up & $0.7474880 \mathrm{I}$ & 0.97721563 \\
\hline miR-489-3p & 0.01149592 & Up & 0.76884233 & 0.98237037 \\
\hline miR-4496 & 0.01149734 & Up & 0.74960999 & 0.97742837 \\
\hline miR-7978 & 0.01150772 & Up & 0.78322993 & 0.98667434 \\
\hline miR-676-3p & 0.01153637 & Up & 0.80272434 & 0.99063213 \\
\hline miR-4650-3p & 0.01157133 & Up & $0.597 \mid 788$ & 0.9386847 I \\
\hline miR-216a-5p & 0.0116056 & Up & 0.6784294 & 0.95648223 \\
\hline miR-5197-3p & 0.01164863 & Up & 0.76952426 & 0.98237037 \\
\hline miR-3I32 & 0.01168148 & Up & $0.7596037 \mid$ & $0.98 \mid 40055$ \\
\hline$m i R-3150 b-5 p$ & 0.011704 & Up & 0.78040012 & 0.98667434 \\
\hline miR-668-5p & 0.01170815 & Up & 0.66036011 & 0.95397723 \\
\hline miR-5092 & 0.01171057 & Up & 0.65400126 & 0.95321673 \\
\hline$m i R-6834-5 p$ & 0.01173243 & Up & 0.63699015 & 0.95096807 \\
\hline miR-3I79 & 0.01173464 & Up & 0.70553052 & 0.96360966 \\
\hline miR-582-3p & 0.01180101 & Up & $0.65549 \mid 23$ & 0.95321673 \\
\hline
\end{tabular}

(Continued) 
Table 9 (Continued).

\begin{tabular}{|c|c|c|c|c|}
\hline miRNAs & Log2FoldChange & Regulation & P-value & P-adj \\
\hline miR-478I-3p & 0.01182106 & Up & $0.7 \mid 425667$ & 0.96505269 \\
\hline $\mathrm{miR}-4502$ & 0.01188395 & Up & 0.66321265 & 0.95510802 \\
\hline miR-3I49 & 0.01193411 & Up & 0.75330525 & 0.97975429 \\
\hline$m i R-47 \mid 2-5 p$ & 0.01193425 & Up & $0.7774 \mid 809$ & 0.985258 \\
\hline miR-3|30-3p & 0.01198522 & Up & 0.72070737 & 0.96558066 \\
\hline miR-4473 & 0.01201285 & $U_{p}$ & 0.61156537 & 0.94386479 \\
\hline miR-3।42 & 0.01202439 & $U_{p}$ & 0.82632704 & 0.99340905 \\
\hline miR-1910-5p & 0.01204555 & Up & 0.86979935 & 0.99898712 \\
\hline miR-5008-5p & 0.01210927 & Up & 0.67184466 & 0.95648223 \\
\hline miR-6769a-3p & 0.01231759 & Up & 0.67377095 & 0.95648223 \\
\hline miR-550b-2-5p & 0.0123235 & $U_{p}$ & 0.6803527 & 0.95756103 \\
\hline miR-132-5p & 0.01239979 & Up & 0.62785584 & $0.949834 I$ \\
\hline miR-377-5p & 0.01243349 & $U_{p}$ & 0.73963553 & 0.97402999 \\
\hline $\operatorname{miR}-3612$ & 0.01246368 & $U_{p}$ & $0.75708 \mid 45$ & $0.98 \mid 40055$ \\
\hline miR-3908 & 0.01253284 & $U_{p}$ & 0.71684988 & 0.96505269 \\
\hline miR-34c-5p & 0.01254468 & $U_{p}$ & $0.70448 \mid 88$ & 0.96360966 \\
\hline$m i R-6765-5 p$ & 0.01255238 & $U_{p}$ & 0.67419733 & 0.95648223 \\
\hline$m i R-4745-3 p$ & 0.01256461 & $U_{p}$ & $0.6989879 \mid$ & 0.96268515 \\
\hline miR-4738-3p & 0.01276161 & Up & 0.59320033 & 0.93770773 \\
\hline$m i R-4638-3 p$ & 0.01277387 & $U_{p}$ & 0.59292589 & 0.93770773 \\
\hline miR-1914-5p & 0.01282594 & $U_{p}$ & 0.73045802 & 0.97193735 \\
\hline miR-129-5p & 0.01283002 & $U_{p}$ & 0.63258914 & 0.95096807 \\
\hline miR-544a & 0.01283799 & $U_{p}$ & $0.746|74| \mid$ & $0.976409 \mid$ \\
\hline miR-579-5p & 0.01285453 & $U_{p}$ & 0.62994592 & 0.95065239 \\
\hline miR-3914 & 0.01286086 & $U_{p}$ & 0.62464601 & 0.94934372 \\
\hline miR-65I-3p & 0.01287047 & $U_{p}$ & 0.69538348 & $0.96 \mid 85982$ \\
\hline miR-6084 & 0.01293593 & $U_{p}$ & 0.65583838 & 0.95321673 \\
\hline miR-18Ib-3p & 0.01306301 & $U_{p}$ & 0.78935621 & 0.98667434 \\
\hline$m i R-410-5 p$ & 0.01310405 & $U_{p}$ & 0.51984245 & 0.91632037 \\
\hline miR-323b-5p & 0.01310415 & Up & 0.69390838 & 0.96137172 \\
\hline miR-7849-3p & 0.0131112 & $U_{p}$ & 0.61849336 & 0.94829596 \\
\hline$m i R-597-3 p$ & 0.01311716 & Up & 0.71900872 & 0.96505269 \\
\hline miR-124-5p & 0.0131234 & $U_{p}$ & 0.79377874 & 0.98694309 \\
\hline miR-363-5p & 0.01318634 & Up & 0.76210555 & 0.98140055 \\
\hline miR-3934-3p & 0.01322045 & $U_{p}$ & 0.67932933 & 0.9566446 \\
\hline miR-3687 & 0.01324644 & $U_{p}$ & 0.59548597 & 0.9386847 I \\
\hline $\operatorname{miR}-3167$ & 0.01333017 & $U_{p}$ & 0.63645436 & 0.95096807 \\
\hline miR-47I0 & 0.01333306 & $U_{p}$ & 0.52798647 & 0.92119547 \\
\hline miR-4307 & 0.01336258 & Up & 0.70485276 & 0.96360966 \\
\hline miR-4775 & 0.01343595 & Up & 0.54052103 & 0.9213181 \\
\hline miR-3928-5p & $0.0135178 \mid$ & $U_{p}$ & 0.80368404 & 0.99063213 \\
\hline miR-92a-I-5p & 0.01356483 & $U_{p}$ & 0.75096762 & 0.97770354 \\
\hline miR-5I7-5p & 0.01361653 & $U_{p}$ & 0.66420343 & 0.95510802 \\
\hline miR-I292-5p & 0.01366842 & $U_{p}$ & 0.63710706 & 0.95096807 \\
\hline miR-202-3p & 0.01377676 & $U_{p}$ & 0.62199514 & $0.9486810 \mathrm{I}$ \\
\hline miR-8056 & 0.01404629 & Up & 0.65642876 & 0.95321673 \\
\hline miR-3936 & 0.01405122 & Up & 0.69131766 & 0.96077402 \\
\hline$m i R-4661-3 p$ & 0.01406232 & $U_{p}$ & 0.53649807 & 0.9213181 \\
\hline miR-448I & $0.014 \mid 4048$ & $U_{p}$ & 0.65872097 & 0.95321673 \\
\hline
\end{tabular}

(Continued) 
Table 9 (Continued).

\begin{tabular}{|c|c|c|c|c|}
\hline miRNAs & Log2FoldChange & Regulation & P-value & P-adj \\
\hline miR-1298-5p & $0.014|48| 4$ & $U_{p}$ & 0.70116334 & 0.96268515 \\
\hline miR-429 & 0.01419675 & Up & $0.578502 \mid 4$ & 0.93436563 \\
\hline miR-4774-3p & 0.0142617 & Up & 0.64602929 & 0.95321673 \\
\hline miR-548ad-5p & $0.0142707 \mid$ & Up & 0.56433017 & 0.93029412 \\
\hline miR-4472 & 0.01428134 & Up & 0.54467602 & 0.92261663 \\
\hline miR-45I7 & 0.01429999 & Up & 0.65536784 & 0.95321673 \\
\hline miR-99b-3p & 0.01433655 & Up & 0.54153182 & 0.92167998 \\
\hline miR-4644 & 0.01436547 & Up & $0.64547 \mid 46$ & 0.95321673 \\
\hline miR-I250-5p & 0.01443761 & Up & 0.53908047 & 0.9213181 \\
\hline miR-372-3p & 0.0144946 & Up & 0.58151827 & 0.93436563 \\
\hline miR-656-3p & 0.01455139 & Up & 0.80755313 & 0.99064883 \\
\hline miR-4640-3p & 0.01456831 & Up & 0.78150428 & 0.98667434 \\
\hline miR-I05-3p & 0.01458433 & Up & 0.55080673 & 0.92349191 \\
\hline miR-4498 & 0.01461732 & Up & 0.60334631 & 0.94089807 \\
\hline miR-3688-5p & 0.01464264 & Up & 0.61124878 & 0.94386479 \\
\hline miR-680I-5p & 0.01464637 & Up & 0.60580933 & 0.94188142 \\
\hline miR-4656 & 0.01466241 & Up & $0.890 \mid 2295$ & $0.998987 / 2$ \\
\hline miR-6856-5p & 0.01469675 & Up & 0.5891102 & 0.93675929 \\
\hline miR-5586-5p & 0.01477128 & Up & 0.67846969 & 0.95648223 \\
\hline miR-6756-3p & 0.0148236 & Up & 0.84494113 & $0.9965428 I$ \\
\hline miR-6729-3p & 0.01488382 & Up & 0.76047908 & $0.98 \mid 40055$ \\
\hline miR-453I & 0.01488447 & Up & 0.59076862 & 0.93675929 \\
\hline miR-3937 & 0.01490706 & Up & 0.73739196 & 0.97354829 \\
\hline miR-3607-5p & 0.01492801 & $U_{p}$ & 0.68988789 & 0.96036263 \\
\hline miR-4744 & 0.01496713 & Up & 0.58205836 & 0.93436563 \\
\hline miR-1537-3p & 0.01497413 & Up & 0.74459697 & 0.97605174 \\
\hline miR-5584-5p & 0.015004 & Up & 0.6644876 & 0.95510802 \\
\hline miR-4778-5p & 0.01507405 & Up & $0.7079 \mid 484$ & 0.96360966 \\
\hline miR-6852-3p & 0.01514938 & Up & $0.630824 I I$ & 0.95086097 \\
\hline miR-938 & 0.01520606 & $U_{p}$ & 0.65801777 & 0.95321673 \\
\hline miR-3177-5p & 0.01524698 & Up & 0.51696969 & 0.91376348 \\
\hline miR-3199 & 0.0152907 & Up & 0.69019057 & 0.96036263 \\
\hline miR-4477a & 0.01533614 & Up & 0.6204992 & 0.94829596 \\
\hline miR-I I $85-2-3 p$ & 0.01534462 & Up & $0.7 \mid 296587$ & 0.96505269 \\
\hline miR-6509-3p & 0.01541891 & Up & 0.73653953 & 0.97354829 \\
\hline miR-6884-5p & 0.01546598 & Up & 0.44533113 & 0.88952595 \\
\hline miR-4765 & 0.01550301 & Up & 0.49293956 & 0.90695643 \\
\hline$m i R-502-5 p$ & 0.01550968 & Up & 0.80976632 & 0.99064883 \\
\hline miR-5588-3p & 0.0155201 & Up & 0.65535348 & 0.95321673 \\
\hline miR-3|9|-3p & 0.01552654 & Up & $0.5555086 \mathrm{I}$ & 0.9264485 \\
\hline miR-47I6-3p & 0.01552688 & Up & $0.88286 \mid 14$ & 0.99898712 \\
\hline miR-4426 & 0.01555663 & Up & $0.6020457 \mid$ & 0.93953261 \\
\hline miR-43II & 0.01562945 & Up & $0.6633234 I$ & 0.95510802 \\
\hline miR-6730-3p & 0.01579544 & Up & 0.63661012 & 0.95096807 \\
\hline$m i R-4796-5 p$ & 0.01579621 & $U_{p}$ & $0.8|253| 5 \mid$ & 0.99064883 \\
\hline miR-378j & 0.01582298 & Up & 0.59044664 & 0.93675929 \\
\hline miR-4276 & 0.01582809 & Up & 0.49987739 & 0.91121111 \\
\hline miR-509I & 0.01587546 & Up & 0.51631719 & 0.91376348 \\
\hline miR-622 & 0.01587548 & Up & 0.65431659 & 0.95321673 \\
\hline
\end{tabular}

(Continued) 
Table 9 (Continued).

\begin{tabular}{|c|c|c|c|c|}
\hline miRNAs & Log2FoldChange & Regulation & P-value & P-adj \\
\hline miR-4477b & 0.01595427 & Up & 0.67510492 & 0.95648223 \\
\hline $\mathrm{miR}-4480$ & 0.01605291 & Up & 0.68121945 & $0.958256 \mid 4$ \\
\hline miR-6730-5p & 0.0160569 & Up & 0.6543226 & 0.95321673 \\
\hline miR-4653-5p & 0.01607326 & Up & 0.60565483 & $0.94|88| 42$ \\
\hline miR-3934-5p & 0.01611412 & Up & 0.64022393 & 0.95096807 \\
\hline miR-30lb-5p & 0.01621443 & $U_{p}$ & 0.61120826 & 0.94386479 \\
\hline miR-4642 & 0.01624775 & $U_{p}$ & 0.68349862 & 0.95853044 \\
\hline$m i R-687 I-3 p$ & 0.01627526 & Up & 0.41542735 & 0.8804535 \\
\hline$m i R-2 I I-5 p$ & 0.01631686 & Up & 0.78984502 & 0.98667434 \\
\hline miR-448 & 0.01636933 & Up & 0.68645874 & 0.96036263 \\
\hline miR-548ai & 0.01646598 & $U_{p}$ & 0.76498329 & $0.98|4768|$ \\
\hline$m i R-6502-3 p$ & 0.01660247 & $U_{p}$ & $0.753 \mid 2865$ & 0.97975429 \\
\hline miR-4430 & 0.01667698 & $U_{p}$ & 0.87860394 & 0.99898712 \\
\hline miR-6740-3p & 0.01668376 & $U_{p}$ & 0.71732029 & 0.96505269 \\
\hline miR-4727-5p & 0.01668725 & $U_{p}$ & $0.59607 \mid 5 I$ & 0.9386847 I \\
\hline miR-5004-3p & 0.01670733 & $U_{p}$ & 0.5998042 & $0.9395326 \mathrm{I}$ \\
\hline miR-5684 & 0.01673479 & $U_{p}$ & $0.7850|76|$ & 0.98667434 \\
\hline miR-5002-3p & 0.01677134 & Up & $0.6464 I 587$ & 0.95321673 \\
\hline miR-I203 & 0.01680127 & Up & $0.6652878 I$ & 0.95557578 \\
\hline miR-369-3p & 0.01686971 & $U_{p}$ & 0.6097989 I & 0.94386479 \\
\hline miR-200a-3p & 0.01701242 & $U_{p}$ & 0.52452216 & 0.91825968 \\
\hline miR-375 & 0.01706379 & $U_{p}$ & 0.55983417 & 0.9276427 \\
\hline miR-30d-5p & 0.01708292 & $U_{p}$ & 0.7998693 & 0.98830004 \\
\hline miR-3689a-3p & 0.01718387 & $U_{p}$ & 0.49300838 & 0.90695643 \\
\hline miR-1909-5p & 0.0172932 & $U_{p}$ & 0.65705658 & 0.95321673 \\
\hline$m i R-4750-3 p$ & 0.01735663 & $U_{p}$ & 0.74220642 & $0.974|933|$ \\
\hline miR-3675-3p & 0.01737469 & $U_{p}$ & 0.80972313 & 0.99064883 \\
\hline miR-765 & 0.01737509 & $U_{p}$ & 0.69856279 & 0.96268515 \\
\hline miR-6775-5p & 0.01746273 & $U_{p}$ & 0.71605815 & 0.96505269 \\
\hline miR-497-3p & 0.0174979 & Up & 0.50358715 & 0.91147569 \\
\hline miR-4500 & 0.01753437 & $U_{p}$ & 0.62783916 & $0.949834 I$ \\
\hline miR-139-3p & 0.01757692 & Up & 0.86938452 & 0.99898712 \\
\hline miR-3194-3p & 0.01763165 & $U_{p}$ & 0.50079992 & 0.91121111 \\
\hline miR-4679 & 0.01782269 & Up & 0.5908509 & 0.93675929 \\
\hline miR-27a-5p & 0.01792569 & $U_{p}$ & 0.56305474 & 0.92970327 \\
\hline miR-1296-5p & 0.01793662 & $U_{p}$ & 0.58790108 & 0.93675929 \\
\hline miR-4755-3p & 0.01795334 & $U_{p}$ & 0.54032429 & 0.9213181 \\
\hline miR-67I-5p & 0.01798175 & $U_{p}$ & 0.91512111 & 0.99898712 \\
\hline$m i R-520 e$ & 0.01806457 & Up & 0.70204427 & 0.96268515 \\
\hline$m i R-670-3 p$ & 0.01811048 & Up & 0.63264444 & 0.95096807 \\
\hline miR-3929 & 0.01811785 & $U_{p}$ & 0.52196244 & 0.91732227 \\
\hline miR-936 & 0.01818701 & $U_{p}$ & 0.50295729 & 0.91147569 \\
\hline$m i R-203 b-5 p$ & 0.0183307 & $U_{p}$ & 0.54804896 & 0.92261663 \\
\hline miR-6776-3p & 0.01834543 & $U_{p}$ & 0.70605908 & 0.96360966 \\
\hline miR-36II & 0.01837621 & $U_{p}$ & 0.62549396 & $0.949834 \mid$ \\
\hline miR-376b-5p & 0.0184051 & Up & 0.58970873 & 0.93675929 \\
\hline$m i R-148 b-5 p$ & 0.01843913 & Up & 0.62829493 & $0.949834 \mathrm{I}$ \\
\hline miR-153-3p & 0.01844682 & $U_{p}$ & 0.60505783 & $0.94|88| 42$ \\
\hline miR-4795-5p & 0.01849838 & $U_{p}$ & 0.62346004 & $0.9492584 \mid$ \\
\hline
\end{tabular}

(Continued) 
Table 9 (Continued).

\begin{tabular}{|c|c|c|c|c|}
\hline miRNAs & Log2FoldChange & Regulation & P-value & P-adj \\
\hline miR-55lb-5p & 0.01851548 & $U_{p}$ & 0.47290103 & 0.89396011 \\
\hline miR-6083 & 0.0185183 & Up & 0.49043732 & 0.90695643 \\
\hline miR-6829-5p & 0.0185316 & Up & 0.68941596 & 0.96036263 \\
\hline miR-1245b-3p & 0.01889982 & Up & 0.59437657 & 0.93829717 \\
\hline miR-323a-5p & 0.01897084 & Up & 0.57029425 & 0.93235129 \\
\hline miR-4635 & 0.01898759 & Up & 0.51382454 & 0.91376348 \\
\hline miR-500I-3p & 0.01900715 & Up & $0.58|237| 3$ & 0.93436563 \\
\hline miR-939-3p & 0.01900859 & Up & $0.605307 \mid$ & $0.94|88| 42$ \\
\hline miR-646 & 0.01904427 & Up & 0.50975211 & 0.91230009 \\
\hline miR-4724-5p & 0.01904875 & Up & 0.46392595 & 0.89396011 \\
\hline miR-673I-3p & 0.01906737 & Up & 0.72346229 & 0.96691758 \\
\hline miR-5I6b-5p & 0.01910255 & Up & 0.48676275 & 0.90650744 \\
\hline miR-6779-3p & 0.01916462 & Up & 0.62653619 & $0.949834 I$ \\
\hline miR-4482-5p & 0.01920295 & Up & $0.5409607 \mid$ & 0.9213181 \\
\hline miR-6823-5p & 0.01922385 & Up & $0.46 \mid 36863$ & 0.89396011 \\
\hline$m i R-680 I-3 p$ & 0.01934473 & Up & 0.71769458 & 0.96505269 \\
\hline$m i R-520 c-3 p$ & 0.01935136 & Up & 0.59704876 & 0.9386847 I \\
\hline miR-68I5-3p & 0.01936625 & Up & $0.76197 \mid 25$ & $0.98 \mid 40055$ \\
\hline miR-65I4-3p & 0.01937438 & Up & 0.4584095 & $0.893 \mid 8606$ \\
\hline miR-686I-5p & 0.01947914 & Up & 0.53968436 & 0.9213181 \\
\hline miR-3II & 0.01953288 & Up & 0.36524879 & 0.8588192 \\
\hline miR-4700-5p & 0.01954516 & Up & $0.3973224 \mid$ & 0.87364718 \\
\hline miR-6795-3p & 0.01964065 & Up & $0.70|47| 27$ & 0.96268515 \\
\hline miR-5698 & 0.01965235 & $U_{p}$ & 0.43976949 & 0.88952595 \\
\hline$m i R-4508$ & 0.01972715 & Up & 0.40121234 & 0.87364718 \\
\hline$m i R-55$ la & 0.01972754 & Up & 0.61849932 & 0.94829596 \\
\hline miR-I 279 & 0.01986827 & Up & 0.59078677 & 0.93675929 \\
\hline miR-3664-5p & 0.019903 & Up & 0.45946349 & $0.8939601 \mathrm{I}$ \\
\hline$m i R-674 I-3 p$ & 0.01992061 & Up & 0.58584764 & 0.93575415 \\
\hline miR-6I30 & 0.02000124 & $U_{p}$ & 0.41972578 & 0.8823614 \\
\hline miR-3|27-5p & 0.02001376 & Up & $0.66753|3|$ & 0.95631006 \\
\hline miR-3973 & 0.02007343 & Up & 0.46691545 & $0.8939601 \mathrm{I}$ \\
\hline miR-6854-5p & 0.02017661 & Up & 0.53693007 & 0.9213181 \\
\hline miR-520a-5p & 0.02025704 & Up & 0.46924916 & $0.8939601 \mathrm{I}$ \\
\hline miR-6805-5p & 0.02026553 & Up & 0.52305157 & 0.9175717 \\
\hline miR-4294 & 0.02033504 & Up & 0.35358085 & 0.8588192 \\
\hline miR-93-3p & 0.02033835 & Up & 0.76260214 & $0.98 \mid 40055$ \\
\hline$m i R-4684-3 p$ & 0.02043351 & Up & 0.43276033 & 0.88833759 \\
\hline$m i R-433-5 p$ & 0.02044078 & Up & 0.37902916 & 0.8649907 I \\
\hline miR-4757-3p & 0.02046866 & Up & 0.55122046 & 0.92349191 \\
\hline miR-506-3p & 0.02048703 & Up & $0.6377734 \mid$ & 0.95096807 \\
\hline miR-517a-3p & 0.02049334 & Up & 0.71781101 & 0.96505269 \\
\hline miR-548ar-5p & 0.02056456 & Up & 0.52876085 & 0.92119547 \\
\hline miR-544b & 0.02064575 & Up & 0.50912217 & 0.91230009 \\
\hline miR-194-3p & 0.02077382 & Up & 0.39578604 & $0.873|074|$ \\
\hline miR-708-3p & 0.02098796 & Up & 0.40708667 & 0.87475982 \\
\hline miR-4764-5p & 0.02118715 & Up & $0.568 \mid 2303$ & 0.9303065 \\
\hline miR-599 & 0.02121295 & Up & 0.43535852 & 0.88941554 \\
\hline$m i R-5003-5 p$ & $0.02|2474|$ & Up & 0.44566007 & 0.88952595 \\
\hline
\end{tabular}

(Continued) 
Table 9 (Continued).

\begin{tabular}{|c|c|c|c|c|}
\hline miRNAs & Log2FoldChange & Regulation & P-value & P-adj \\
\hline miR-1226-3p & 0.02132186 & Up & 0.53242448 & 0.9213181 \\
\hline miR-325 & 0.02133066 & Up & 0.52051665 & 0.91687991 \\
\hline miR-548ba & $0.02148 \mid 62$ & Up & 0.44559008 & 0.88952595 \\
\hline miR-6874-5p & 0.02148592 & Up & 0.54504845 & 0.92261663 \\
\hline miR-7I5I-5p & 0.02163544 & Up & 0.30004361 & 0.83872374 \\
\hline miR-499a-5p & 0.02164053 & Up & 0.5426569 & 0.9222099 \\
\hline miR-449I & 0.02166487 & Up & $0.47 \mid 54073$ & $0.893960 \mathrm{II}$ \\
\hline$m i R-518 f-3 p$ & 0.02169151 & Up & 0.6831004 & 0.95853044 \\
\hline miR-68II-3p & 0.02180674 & Up & 0.60084548 & 0.93953261 \\
\hline miR-3।84-3p & 0.02185842 & Up & 0.5673034 & 0.9303065 \\
\hline miR-6825-3p & 0.02186425 & Up & 0.65344952 & 0.95321673 \\
\hline$m i R-556-5 p$ & 0.02187098 & Up & 0.63388765 & 0.95096807 \\
\hline miR-3190-3p & $0.0220 \mid 55 I$ & Up & 0.50097307 & 0.91121111 \\
\hline miR-5693 & $0.02206 \mid 22$ & Up & 0.50386992 & 0.91147569 \\
\hline miR-499b-3p & 0.02210218 & Up & 0.58323296 & 0.93436563 \\
\hline miR-5700 & 0.02218527 & Up & 0.58994637 & 0.93675929 \\
\hline miR-4787-5p & 0.02221382 & Up & 0.65864707 & 0.95321673 \\
\hline miR-4676-5p & 0.02222161 & Up & 0.47930937 & 0.90243596 \\
\hline miR-6820-5p & 0.0222336 & Up & $0.583 \mid 9465$ & 0.93436563 \\
\hline miR-4804-5p & 0.02233079 & Up & 0.49202453 & 0.90695643 \\
\hline miR-380-5p & $0.02237 \mid 46$ & Up & 0.51536985 & 0.91376348 \\
\hline miR-6742-3p & 0.02237604 & Up & 0.58352406 & 0.93436563 \\
\hline miR-6833-5p & $0.0224395 I$ & $U_{p}$ & 0.50926238 & 0.91230009 \\
\hline miR-687I-5p & 0.02248366 & Up & $0.42648 \mid 47$ & 0.88249386 \\
\hline miR-19b-2-5p & 0.02252117 & Up & 0.64845823 & 0.95321673 \\
\hline miR-367I & 0.0225583 & Up & 0.56831954 & 0.9303065 \\
\hline miR-4447 & 0.02263427 & Up & 0.43937869 & 0.88952595 \\
\hline miR-525-3p & 0.02266101 & Up & 0.62137369 & 0.94829596 \\
\hline miR-686I-3p & 0.02274475 & Up & 0.58950393 & 0.93675929 \\
\hline miR-I 275 & $0.02289|2|$ & Up & 0.91458073 & 0.99898712 \\
\hline miR-5।8e-5p & 0.02294105 & Up & 0.45597788 & 0.89182889 \\
\hline miR-I287-3p & 0.02310958 & Up & 0.45535913 & 0.891297 \\
\hline miR-3617-5p & 0.02323344 & Up & 0.64968436 & 0.95321673 \\
\hline miR-6783-5p & 0.0233775 & Up & 0.43556048 & 0.88941554 \\
\hline miR-7I60-5p & 0.02343496 & Up & 0.5065038 & 0.912181 \\
\hline miR-5579-5p & 0.02344538 & Up & 0.55670065 & 0.92696334 \\
\hline miR-6760-3p & 0.02347367 & Up & $0.73868 \mid 43$ & 0.97402999 \\
\hline miR-7I54-3p & 0.02348532 & Up & 0.45486744 & 0.891297 \\
\hline miR-4292 & 0.02355298 & Up & 0.4574619 & $0.8924444 I$ \\
\hline$m i R-4753-5 p$ & 0.02367013 & Up & 0.42523878 & $0.88236 \mid 4$ \\
\hline miR-47I5-3p & 0.02377472 & $U_{p}$ & 0.67202426 & 0.95648223 \\
\hline miR-2467-5p & 0.02382854 & $U_{p}$ & 0.48887473 & 0.90656383 \\
\hline miR-8054 & 0.02383556 & Up & $0.44 \mid 48897$ & 0.88952595 \\
\hline $\operatorname{miR}-4313$ & 0.02387812 & Up & 0.69997027 & 0.96268515 \\
\hline miR-676I-5p & 0.02391023 & Up & 0.46096324 & 0.89396011 \\
\hline miR-I 272 & 0.02407068 & Up & 0.40836297 & 0.87603742 \\
\hline miR-942-3p & 0.02408566 & Up & 0.40786434 & 0.8756987 \\
\hline miR-425I & 0.02411161 & Up & 0.54820586 & 0.92261663 \\
\hline miR-210-5p & 0.02422365 & $U_{p}$ & 0.47292638 & 0.89396011 \\
\hline
\end{tabular}

(Continued) 
Table 9 (Continued).

\begin{tabular}{|c|c|c|c|c|}
\hline miRNAs & Log2FoldChange & Regulation & P-value & P-adj \\
\hline miR-3927-3p & 0.02423045 & $U_{p}$ & 0.6019432 & 0.93953261 \\
\hline miR-553 & 0.02423263 & Up & $0.5605792 I$ & 0.92768099 \\
\hline miR-6869-3p & 0.02424035 & Up & 0.52250599 & 0.91732227 \\
\hline miR-4999-5p & 0.02427566 & Up & 0.49857628 & 0.91032268 \\
\hline miR-324-3p & 0.02431653 & Up & 0.71762607 & 0.96505269 \\
\hline miR-1226-5p & 0.02438006 & Up & $0.4 \mid 293737$ & 0.8804535 \\
\hline miR-3619-3p & $0.0244037 \mid$ & Up & 0.44753193 & 0.88952595 \\
\hline miR-20b-3p & 0.02441946 & Up & 0.39889277 & 0.87364718 \\
\hline miR-4704-5p & 0.02442544 & Up & 0.46980283 & 0.89396011 \\
\hline miR-504-5p & 0.0244978 & Up & 0.4506903 & 0.88964976 \\
\hline miR-4746-5p & 0.02467118 & Up & 0.35223335 & 0.8588192 \\
\hline$m i R-522-3 p$ & 0.02469827 & Up & 0.55698033 & 0.92696334 \\
\hline miR-4804-3p & 0.024709 & Up & 0.36485338 & 0.8588192 \\
\hline miR-446I & $0.0247,6583$ & Up & 0.55726006 & 0.92696334 \\
\hline miR-138-5p & $0.0247987 \mid$ & Up & 0.48787964 & 0.90656383 \\
\hline$m i R-548 f-3 p$ & 0.02480375 & Up & 0.6616726 & 0.95510802 \\
\hline miR-5002-5p & 0.02480824 & Up & 0.32490062 & 0.85489649 \\
\hline $\mathrm{miR}-378 \mathrm{e}$ & 0.0248163 & Up & 0.44638568 & 0.88952595 \\
\hline miR-555 & 0.02482369 & Up & $0.4733437 \mid$ & $0.893960 \mathrm{II}$ \\
\hline miR-43I5 & 0.02486687 & Up & 0.49577156 & 0.90944534 \\
\hline miR-182-3p & 0.02490835 & Up & 0.4216688 & 0.8823614 \\
\hline miR-6780a-5p & 0.02508612 & Up & $0.65978 \mid 53$ & 0.95397723 \\
\hline miR-I53-5p & 0.02513058 & Up & $0.50956 \mid 46$ & 0.91230009 \\
\hline miR-6774-5p & 0.0252508 & $U_{p}$ & 0.38578973 & $0.8696 \mid 208$ \\
\hline$m i R-372-5 p$ & 0.025309 & Up & 0.40063252 & 0.87364718 \\
\hline miR-3I59 & 0.02532184 & Up & 0.4205062 & 0.8823614 \\
\hline miR-4467 & 0.02533318 & Up & 0.37976904 & 0.8649907 I \\
\hline miR-3I 68 & 0.02534439 & Up & 0.4408406 & 0.88952595 \\
\hline miR-6889-3p & 0.02542975 & Up & $0.79 \mid 27667$ & 0.98667434 \\
\hline miR-3I50b-3p & 0.02552629 & $U_{p}$ & 0.4247934 I & 0.8823614 \\
\hline$m i R-36 \mid 4-3 p$ & 0.02580515 & Up & $0.3|2463|$ & 0.8475125 \\
\hline miR-4724-3p & $0.025877 \mid 3$ & Up & $0.553 \mid 4262$ & 0.92384946 \\
\hline miR-3679-3p & $0.0259|2| 3$ & Up & 0.71514096 & 0.96505269 \\
\hline miR-4694-3p & 0.0260043 & Up & $0.2976 \mid 764$ & 0.83872374 \\
\hline miR-4252 & 0.02617889 & Up & 0.41182256 & 0.8793356 \\
\hline miR-4774-5p & 0.02618218 & Up & 0.37549511 & 0.8649907 I \\
\hline miR-548p & 0.02618724 & Up & 0.39523775 & $0.873|074|$ \\
\hline$m i R-65 \mid 4-5 p$ & 0.02620675 & Up & 0.37788582 & 0.8649907 I \\
\hline miR-4534 & 0.02621799 & Up & 0.51688833 & 0.91376348 \\
\hline miR-67I5a-3p & 0.02624311 & Up & $0.386 \mid 4243$ & $0.8696 / 208$ \\
\hline miR-3920 & 0.02624926 & Up & 0.69772176 & 0.96250398 \\
\hline miR-7|I3-5p & 0.02634294 & Up & 0.27562149 & $0.83|0854|$ \\
\hline miR-4277 & 0.02639359 & Up & 0.39668404 & 0.87358867 \\
\hline miR-208b-5p & 0.02643533 & Up & 0.53329665 & 0.9213181 \\
\hline$m i R-449 c-3 p$ & 0.02644331 & $U_{p}$ & 0.29568464 & 0.83872374 \\
\hline miR-6I 28 & 0.02667642 & Up & 0.50234964 & 0.91147569 \\
\hline miR-548n & 0.02670062 & Up & 0.31152037 & $0.8475 \mid 25$ \\
\hline miR-6783-3p & $0.0268 \mid 328$ & $U_{p}$ & 0.33060759 & 0.85489649 \\
\hline miR-4427 & $0.0268 I 8 I$ & Up & 0.43559298 & $0.8894 I 554$ \\
\hline
\end{tabular}

(Continued) 
Table 9 (Continued).

\begin{tabular}{|c|c|c|c|c|}
\hline miRNAs & Log2FoldChange & Regulation & P-value & P-adj \\
\hline miR-6802-5p & 0.02693858 & Up & $0.34|402| 8$ & 0.85516921 \\
\hline miR-4529-5p & 0.02696507 & Up & 0.5346817 & 0.9213181 \\
\hline miR-I37 & 0.02707829 & Up & 0.45509436 & 0.891297 \\
\hline miR-4440 & 0.02709012 & Up & 0.34362873 & 0.85823697 \\
\hline miR-75I5 & 0.02711302 & Up & $0.3089 \mid 275$ & 0.84601313 \\
\hline miR-7I54-5p & $0.027 \mid 424$ & Up & 0.37609847 & 0.8649907 I \\
\hline miR-675I-3p & 0.02718007 & Up & 0.5677343 & 0.9303065 \\
\hline miR-642a-5p & 0.02722384 & Up & $0.443|776|$ & 0.88952595 \\
\hline miR-6508-3p & 0.02726898 & Up & 0.54595956 & 0.92261663 \\
\hline $\operatorname{miR}-620$ & $0.0272725 I$ & Up & 0.49055899 & 0.90695643 \\
\hline miR-I 293 & 0.02763147 & Up & 0.35398018 & 0.8588192 \\
\hline let-7e-3p & 0.02772595 & Up & 0.44949219 & 0.88964976 \\
\hline miR-4740-3p & 0.02784092 & Up & 0.43135925 & 0.88759074 \\
\hline miR-548i & 0.02787387 & Up & 0.49872931 & 0.91032268 \\
\hline miR-103a-2-5p & 0.02787897 & Up & 0.51371703 & 0.91376348 \\
\hline miR-I273d & 0.02792954 & $U_{p}$ & 0.26610662 & 0.82438552 \\
\hline miR-4747-5p & 0.02795079 & Up & 0.31530614 & 0.84917098 \\
\hline miR-6749-3p & 0.02808208 & Up & 0.34686008 & 0.8588192 \\
\hline miR-442I & 0.02826019 & Up & 0.27677993 & $0.83 I 0854 \mid$ \\
\hline miR-483-5p & 0.02826129 & Up & 0.6279789 & $0.949834 I$ \\
\hline$m i R-6 / 34$ & 0.02834864 & Up & 0.38783827 & $0.87 \mid 28003$ \\
\hline miR-1294 & 0.02837677 & Up & 0.26192077 & 0.81989814 \\
\hline miR- 1257 & 0.02865524 & $U_{p}$ & 0.3398032 & 0.85489649 \\
\hline miR-6509-5p & 0.02865937 & Up & 0.38183201 & 0.86612247 \\
\hline $\operatorname{miR}-2909$ & $0.0288547 \mid$ & Up & 0.40484157 & 0.87475982 \\
\hline miR-202-5p & 0.02889478 & Up & 0.54327852 & 0.9222099 \\
\hline miR-4786-3p & 0.02906531 & Up & 0.4179162 & 0.88181004 \\
\hline miR-633 & 0.02906807 & Up & 0.34516107 & 0.8588192 \\
\hline miR-4736 & 0.02909211 & Up & 0.46430733 & 0.89396011 \\
\hline miR-548ao-3p & 0.0292578 & Up & 0.41656785 & 0.8804535 \\
\hline miR-6836-3p & 0.0293377 & Up & $0.5334840 \mathrm{I}$ & 0.9213181 \\
\hline$m i R-525-5 p$ & 0.02937872 & Up & $0.2264556 \mathrm{I}$ & 0.79102241 \\
\hline$m i R-125 I-5 p$ & 0.02938206 & Up & 0.38217233 & 0.86612247 \\
\hline miR-1306-3p & 0.02940317 & Up & 0.39910924 & 0.87364718 \\
\hline miR-|23I & $0.02946 \mid 45$ & Up & 0.23748454 & 0.80380192 \\
\hline miR-4302 & 0.02947784 & Up & 0.52691064 & $0.92057 \mid 28$ \\
\hline$m i R-485-5 p$ & 0.02948993 & $U_{p}$ & $0.377777 I I$ & 0.8649907| \\
\hline$m i R-56 I-3 p$ & 0.02950935 & Up & 0.50098883 & 0.91121111 \\
\hline$m i R-892 c-5 p$ & 0.02953377 & Up & 0.40024642 & 0.87364718 \\
\hline miR-7I58-3p & 0.02955477 & Up & $0.3|2856| I$ & 0.8475125 \\
\hline$m i R-3 \mid 24-3 p$ & 0.02959642 & Up & 0.19296399 & 0.7799749 I \\
\hline miR-4476 & 0.02966598 & $U_{p}$ & 0.53982754 & 0.9213181 \\
\hline miR-5195-3p & 0.02973844 & Up & 0.3286761 & 0.85489649 \\
\hline miR-6798-5p & 0.02986807 & Up & 0.53816839 & 0.9213181 \\
\hline miR-474I & 0.02987025 & $U_{p}$ & 0.63334498 & 0.95096807 \\
\hline miR-3620-5p & 0.02992468 & Up & 0.30788948 & 0.84538031 \\
\hline miR-3II6 & 0.03005477 & Up & $0.25045|4|$ & $0.807|5| 25$ \\
\hline miR-7973 & 0.03005652 & Up & 0.32648339 & 0.85489649 \\
\hline miR-500I-5p & 0.0300737 & $U_{p}$ & 0.55332437 & 0.92384946 \\
\hline
\end{tabular}

(Continued) 
Table 9 (Continued).

\begin{tabular}{|c|c|c|c|c|}
\hline miRNAs & Log2FoldChange & Regulation & P-value & P-adj \\
\hline miR-359l-5p & 0.03027396 & $U_{p}$ & $0.25|23| 75$ & $0.807 / 5125$ \\
\hline miR-6788-5p & 0.03031197 & Up & 0.25476983 & 0.81031406 \\
\hline miR-4770 & 0.03031384 & Up & 0.17626762 & 0.77062416 \\
\hline miR-4722-5p & 0.03041055 & Up & 0.27028076 & 0.83107769 \\
\hline miR-670-5p & 0.03047355 & Up & 0.20259859 & 0.7799749 I \\
\hline miR-468I & 0.03050059 & Up & $0.2009 \mid 977$ & 0.7799749 I \\
\hline$m i R-2 \mid 8-I-3 p$ & 0.03055934 & Up & 0.28342391 & 0.83193599 \\
\hline miR-570-3p & 0.0306498 & Up & 0.2948952 & 0.83872374 \\
\hline miR-653-3p & 0.03065132 & Up & $0.392|247|$ & $0.8728 \mid 803$ \\
\hline miR-6076 & 0.03066423 & Up & $0.82,336,926$ & 0.99252298 \\
\hline miR-I587 & 0.0306804 & Up & 0.68602982 & 0.96029228 \\
\hline miR-47I 7-3p & 0.03073293 & Up & 0.218466 & 0.78840757 \\
\hline miR-548ap-5p & 0.03080711 & Up & 0.44476972 & 0.88952595 \\
\hline miR-4445-5p & 0.03089658 & Up & 0.272994 & 0.83107769 \\
\hline miR-6754-3p & 0.03094721 & Up & 0.34121026 & $0.855 \mid 692 I$ \\
\hline miR-586 & 0.03105401 & Up & 0.43070548 & 0.88759074 \\
\hline miR-943 & 0.03113862 & Up & $0.14257 \mid 13$ & $0.7537626 \mathrm{I}$ \\
\hline miR-3972 & 0.03114344 & Up & 0.29560155 & 0.83872374 \\
\hline miR-44I9a & 0.03130364 & Up & $0.36743 \mid 34$ & 0.86195734 \\
\hline miR-6859-3p & 0.031345 & Up & 0.41109277 & 0.8793356 \\
\hline miR-302a-5p & 0.03155607 & Up & $0.405 \mid 9275$ & 0.87475982 \\
\hline miR-548au-3p & 0.03161619 & Up & 0.4213246 & 0.8823614 \\
\hline$m i R-4659 a-3 p$ & 0.03161724 & Up & 0.4496733 & 0.88964976 \\
\hline miR-6820-3p & 0.03164575 & $U_{p}$ & 0.534798 & 0.9213181 \\
\hline miR-203b-3p & 0.0317454 & Up & 0.30313818 & 0.83872374 \\
\hline miR-6720-3p & $0.03|7704|$ & Up & $0.343 \mid 4084$ & $0.857852 I$ \\
\hline miR-645 & 0.03182286 & Up & 0.40658308 & 0.87475982 \\
\hline$m i R-514 b-3 p$ & 0.03184083 & Up & 0.34095019 & $0.855 \mid 692 I$ \\
\hline miR-759 & 0.0318701 & Up & 0.44743825 & 0.88952595 \\
\hline miR-5I88 & 0.03190043 & $U_{p}$ & 0.3645565 I & 0.8588192 \\
\hline miR-47I7-5p & 0.03219936 & Up & 0.3201688 & 0.85046325 \\
\hline miR-5702 & 0.03233689 & Up & 0.27668467 & $0.83|0854|$ \\
\hline miR-548bb-5p & 0.03234506 & Up & 0.17156305 & 0.77062416 \\
\hline miR-677I-5p & 0.03246089 & Up & $0.2303588 I$ & 0.79466058 \\
\hline miR-3672 & 0.03253566 & Up & 0.50166291 & 0.91147569 \\
\hline miR-466 & 0.03254509 & Up & $0.46426 \mid 45$ & $0.893960 \mathrm{II}$ \\
\hline miR-4533 & 0.03257163 & Up & $0.2438257 \mid$ & $0.807|5| 25$ \\
\hline miR-6760-5p & 0.03259034 & Up & 0.83963668 & $0.9965428 \mathrm{I}$ \\
\hline miR-3I55a & 0.03263465 & Up & 0.24121568 & $0.806 \mid 4344$ \\
\hline miR-8057 & 0.0326443 & Up & 0.22658673 & $0.79|0224|$ \\
\hline miR-4425 & 0.03265373 & Up & 0.16032466 & $0.76082 \mid 42$ \\
\hline$m i R-503-5 p$ & $0.03279|3|$ & Up & 0.8542697 & 0.99898712 \\
\hline miR-874-3p & 0.03282655 & Up & 0.84803079 & $0.9965428 \mathrm{I}$ \\
\hline$m i R-542-3 p$ & 0.03283932 & Up & 0.57655026 & 0.93390911 \\
\hline miR-3|2I-3p & 0.03285515 & Up & 0.1695905 & 0.77062416 \\
\hline miR-1910-3p & 0.03289378 & Up & 0.41332006 & 0.8804535 \\
\hline$m i R-552-3 p$ & $0.03299 \mid 45$ & Up & 0.31753409 & 0.84917098 \\
\hline miR-7843-3p & 0.03299986 & Up & 0.36155796 & 0.8588192 \\
\hline miR-6792-5p & 0.03301146 & Up & 0.29107891 & 0.83872374 \\
\hline
\end{tabular}

(Continued) 
Table 9 (Continued).

\begin{tabular}{|c|c|c|c|c|}
\hline miRNAs & Log2FoldChange & Regulation & P-value & P-adj \\
\hline miR-5000-3p & 0.03312279 & Up & $0.20 I 7744 \mid$ & 0.7799749 I \\
\hline miR-II 93 & $0.033|804|$ & Up & 0.11602809 & 0.74226488 \\
\hline miR-3942-3p & 0.03318391 & Up & 0.49335607 & 0.90695643 \\
\hline miR-6845-5p & 0.03321475 & Up & 0.46960894 & $0.8939601 \mathrm{I}$ \\
\hline miR-889-5p & 0.03324309 & Up & 0.28522198 & 0.83289761 \\
\hline miR-|47| & 0.033333836 & $U_{p}$ & 0.31115147 & $0.8475 \mid 25$ \\
\hline miR-585-5p & 0.03336345 & $U_{p}$ & $0.362|328|$ & 0.8588192 \\
\hline miR-65।3-5p & 0.03337839 & Up & 0.29886864 & 0.83872374 \\
\hline $\operatorname{miR}-8074$ & 0.03352439 & Up & 0.35286528 & 0.8588192 \\
\hline miR-3907 & 0.03355366 & Up & 0.31295306 & 0.8475125 \\
\hline miR-3I70 & 0.03361297 & $U_{p}$ & 0.28328304 & 0.83193599 \\
\hline miR-476I-3p & 0.03364893 & Up & 0.3031921 & 0.83872374 \\
\hline miR-6887-5p & 0.03369513 & $U_{p}$ & 0.59653264 & $0.9386847 \mid$ \\
\hline miR-4695-3p & 0.03374892 & $U_{p}$ & 0.33789055 & 0.85489649 \\
\hline miR-487a-5p & 0.03377349 & $U_{p}$ & $0.4636941 I$ & 0.89396011 \\
\hline miR-47|4-5p & 0.03387409 & $U_{p}$ & 0.50397158 & 0.91147569 \\
\hline miR-3942-5p & 0.03395729 & $U_{p}$ & 0.42275918 & $0.88236 \mid 4$ \\
\hline miR-8088 & 0.03395917 & Up & 0.12762754 & 0.74892227 \\
\hline miR-4326 & 0.03399485 & Up & $0.3947 / 249$ & $0.8731074 \mid$ \\
\hline miR-I55-5p & 0.03404459 & $U_{p}$ & 0.80932045 & 0.99064883 \\
\hline miR-3690 & 0.03409675 & $U_{p}$ & 0.39488925 & $0.8731074 \mid$ \\
\hline miR-4685-3p & 0.03416231 & $U_{p}$ & $0.4576816 I$ & $0.8924444 I$ \\
\hline miR-1302 & 0.03425043 & $U_{p}$ & 0.22811873 & 0.79102241 \\
\hline miR-7-2-3p & $0.0344046 \mathrm{I}$ & $U_{p}$ & $0.3315405 I$ & 0.85489649 \\
\hline miR-4677-3p & $0.0344 I 566$ & $U_{p}$ & 0.33771595 & 0.85489649 \\
\hline miR-6754-5p & 0.03443972 & $U_{p}$ & 0.25391716 & 0.81031406 \\
\hline miR-3660 & 0.03456359 & $U_{p}$ & 0.21524655 & 0.7853205 \\
\hline miR-8073 & 0.03459958 & $U_{p}$ & 0.30226843 & 0.83872374 \\
\hline miR-36I4-5p & 0.03464852 & $U_{p}$ & 0.42573079 & $0.88236 \mid 4$ \\
\hline miR-5009-5p & 0.03469731 & Up & 0.2813402 & $0.83|0854|$ \\
\hline miR-4766-3p & 0.03472279 & Up & 0.17886448 & 0.77062416 \\
\hline$m i R-4664-3 p$ & 0.0347848 & Up & 0.42348632 & $0.88236 \mid 4$ \\
\hline miR-4296 & 0.03478611 & $U_{p}$ & 0.28417496 & 0.83193599 \\
\hline miR-684I-5p & 0.03480077 & Up & 0.36207326 & 0.8588192 \\
\hline miR-4699-3p & $0.03485|9|$ & $U_{p}$ & 0.2789268 & $0.8310854 \mid$ \\
\hline miR-105-5p & 0.03492293 & $U_{p}$ & 0.43276212 & 0.88833759 \\
\hline miR-1229-5p & 0.0350583 & $U_{p}$ & 0.70680678 & 0.96360966 \\
\hline miR-I 205 & 0.03512762 & $U_{p}$ & 0.17645303 & 0.77062416 \\
\hline miR-8082 & 0.03537106 & Up & 0.28859008 & 0.83439474 \\
\hline miR-6778-3p & 0.03537593 & Up & 0.35862587 & 0.8588192 \\
\hline miR-8075 & 0.03548293 & $U_{p}$ & 0.23935284 & 0.80380192 \\
\hline miR-520d-3p & 0.03557657 & $U_{p}$ & 0.20689493 & 0.78263372 \\
\hline miR-5 I87-5p & 0.0356064 I & $U_{p}$ & 0.19413706 & 0.7799749 I \\
\hline miR-6736-3p & 0.03566937 & $U_{p}$ & 0.29870046 & 0.83872374 \\
\hline miR-548at-5p & 0.03573935 & Up & 0.30468684 & 0.83927672 \\
\hline miR-557I-3p & 0.03574804 & Up & 0.25145867 & 0.80715125 \\
\hline miR-7853-5p & 0.0357678 & Up & 0.1331583 & 0.74892227 \\
\hline miR-29b-2-5p & 0.03586254 & $U_{p}$ & 0.30415974 & 0.83872374 \\
\hline miR-7844-5p & 0.03587377 & $U_{p}$ & 0.22386382 & $0.7910224 \mid$ \\
\hline
\end{tabular}

(Continued) 
Table 9 (Continued).

\begin{tabular}{|c|c|c|c|c|}
\hline miRNAs & Log2FoldChange & Regulation & P-value & P-adj \\
\hline miR-196a-3p & 0.03601832 & $U_{p}$ & 0.17803967 & 0.77062416 \\
\hline miR-4693-3p & 0.03607285 & Up & 0.19938898 & 0.7799749 I \\
\hline miR-496 & 0.03608936 & Up & 0.32422293 & 0.85489649 \\
\hline miR-6894-5p & 0.03610148 & Up & 0.18941797 & 0.7799749 I \\
\hline miR-7I57-3p & 0.03625689 & Up & 0.18990966 & 0.7799749 I \\
\hline miR-19|4-3p & 0.03627463 & Up & 0.62987625 & 0.95065239 \\
\hline miR-6860 & 0.03627669 & Up & 0.14797885 & $0.7537626 \mathrm{I}$ \\
\hline miR-6750-5p & 0.03627802 & Up & 0.2160179 & 0.7853205 \\
\hline miR-34b-3p & 0.03637661 & Up & 0.40605985 & 0.87475982 \\
\hline miR-6886-5p & 0.0365227 & Up & 0.27957753 & $0.83|0854|$ \\
\hline miR-I245a & 0.03668535 & Up & $0.4366286 I$ & 0.88952595 \\
\hline miR-4720-5p & 0.03681833 & Up & 0.13035282 & 0.74892227 \\
\hline miR-122-5p & $0.03687|7|$ & Up & 0.14472276 & $0.7537626 \mathrm{I}$ \\
\hline miR-1909-3p & 0.03687721 & Up & 0.1819375 & 0.77344537 \\
\hline miR-I 243 & 0.0369511 & Up & 0.34756238 & 0.8588192 \\
\hline miR-6848-5p & 0.03696994 & Up & $0.2724088 I$ & 0.83107769 \\
\hline miR-6736-5p & 0.03706158 & Up & 0.32377062 & 0.85489649 \\
\hline miR-3679-5p & 0.03734236 & Up & 0.73754807 & 0.97354829 \\
\hline miR-4285 & 0.03735561 & Up & $0.208|406|$ & 0.78263372 \\
\hline$m i R-56 I-5 p$ & 0.03752054 & Up & $0.2937964 \mid$ & 0.83872374 \\
\hline miR-6882-3p & 0.03761332 & Up & 0.15777935 & $0.759885 I 5$ \\
\hline miR-4474-5p & 0.03763317 & Up & 0.30910752 & 0.84601313 \\
\hline miR-I236-3p & $0.0377346 \mid$ & Up & 0.37922698 & 0.8649907 I \\
\hline miR-568Ia & 0.03801933 & $U_{p}$ & 0.24815225 & $0.807 / 5 I 25$ \\
\hline miR-39l0 & 0.03806447 & Up & 0.28096822 & $0.83|0854|$ \\
\hline miR-3678-3p & 0.03807823 & Up & 0.36396818 & 0.8588192 \\
\hline miR-6838-5p & 0.03811724 & Up & 0.13604868 & $0.7537626 \mathrm{I}$ \\
\hline miR-466I-5p & $0.0382028 I$ & Up & 0.23108332 & $0.7950256 \mathrm{I}$ \\
\hline miR-598-5p & 0.03830432 & Up & 0.15547245 & 0.75588563 \\
\hline miR-6825-5p & 0.03837002 & $U_{p}$ & 0.18588078 & 0.77677007 \\
\hline miR-579-3p & 0.0383978 & Up & 0.10369361 & 0.74226488 \\
\hline miR-6768-5p & 0.03840136 & Up & 0.29653583 & 0.83872374 \\
\hline miR-607 & 0.03844626 & Up & 0.43070991 & 0.88759074 \\
\hline miR-5I4b-5p & 0.03847919 & Up & 0.12575112 & 0.74465526 \\
\hline miR-376c-5p & 0.03868361 & Up & 0.3607905 & 0.8588192 \\
\hline miR-677I-3p & 0.03868435 & Up & 0.39572746 & $0.873|074|$ \\
\hline miR-67I-3p & 0.03879676 & Up & 0.4395678 & 0.88952595 \\
\hline miR-7974 & 0.0388885 & Up & 0.20181095 & 0.7799749 I \\
\hline miR-523-3p & 0.03900629 & Up & 0.14829019 & $0.7537626 \mathrm{I}$ \\
\hline miR-4760-3p & 0.03917813 & Up & 0.39896188 & 0.87364718 \\
\hline miR-675-3p & 0.03922992 & Up & 0.34598835 & 0.8588192 \\
\hline miR-I233-3p & 0.03932368 & Up & 0.49662437 & $0.9097 \mid 108$ \\
\hline miR-500a-3p & 0.03935554 & Up & 0.61879455 & 0.94829596 \\
\hline miR-3I80 & $0.0394|22|$ & Up & 0.16395796 & 0.7689269 I \\
\hline miR-33b-5p & 0.03959734 & Up & 0.31606259 & 0.84917098 \\
\hline miR-47II-3p & 0.03964566 & Up & 0.25101393 & $0.807|5| 25$ \\
\hline miR-4750-5p & $0.0397 \mid 147$ & Up & 0.08287334 & 0.69839839 \\
\hline miR-367-3p & 0.03984679 & $U_{p}$ & 0.25183159 & $0.807|5| 25$ \\
\hline miR-323b-3p & 0.03990502 & Up & 0.17721599 & 0.77062416 \\
\hline
\end{tabular}

(Continued) 
Table 9 (Continued).

\begin{tabular}{|c|c|c|c|c|}
\hline miRNAs & Log2FoldChange & Regulation & P-value & P-adj \\
\hline miR-|32| & 0.04002516 & Up & 0.13375694 & 0.74892227 \\
\hline miR-18a-3p & 0.04010365 & Up & 0.37562221 & $0.8649907 \mid$ \\
\hline miR-6072 & 0.04010963 & Up & 0.33186263 & 0.85489649 \\
\hline miR-449b-3p & 0.0401152 & Up & $0.1940 \mid 484$ & 0.7799749 I \\
\hline miR-3173-3p & 0.04036297 & Up & $0.2482506 I$ & 0.80715125 \\
\hline miR-3156-5p & 0.04040939 & Up & 0.38153022 & $0.866 \mid 2247$ \\
\hline miR-447I & 0.04042235 & Up & 0.33978286 & 0.85489649 \\
\hline miR-580-5p & $0.040565 I 5$ & Up & 0.32836793 & 0.85489649 \\
\hline miR-6079 & 0.04060488 & Up & 0.25071618 & $0.807|5| 25$ \\
\hline miR-5699-5p & 0.04066678 & Up & $0.26624 I 24$ & 0.82438552 \\
\hline$m i R-4272$ & 0.04067212 & Up & 0.39660658 & 0.87358867 \\
\hline miR-548h-3p & $0.040735 \mathrm{I}$ & Up & 0.18575176 & 0.77677007 \\
\hline miR-6876-3p & 0.04074924 & Up & 0.16606324 & $0.769|508|$ \\
\hline miR-892b & 0.04078216 & Up & 0.30408928 & 0.83872374 \\
\hline miR-6807-5p & 0.04078605 & Up & 0.53160743 & 0.9213181 \\
\hline $\operatorname{miR}-45 \mid 8$ & 0.04082069 & $U_{p}$ & 0.39033207 & $0.8728 \mid 803$ \\
\hline miR-100-3p & 0.04085695 & Up & 0.45761716 & $0.8924444 \mathrm{I}$ \\
\hline miR-4327 & 0.04089764 & Up & 0.40025816 & 0.87364718 \\
\hline miR-6765-3p & 0.04091817 & Up & 0.51555157 & 0.91376348 \\
\hline miR-380-3p & 0.04097196 & Up & 0.35794546 & 0.8588192 \\
\hline miR-6846-3p & 0.04101375 & Up & 0.27666692 & $0.83|0854|$ \\
\hline miR-808I & 0.04118224 & Up & 0.29757035 & 0.83872374 \\
\hline$m i R-4438$ & $0.04 \mid 25884$ & $U_{p}$ & 0.14766533 & $0.7537626 \mathrm{I}$ \\
\hline miR-559I-5p & 0.04127629 & Up & 0.13916892 & $0.7537626 \mathrm{I}$ \\
\hline miR-362I & 0.04129921 & Up & 0.15845074 & $0.759885 I 5$ \\
\hline miR-3I88 & 0.04129974 & Up & 0.18757745 & 0.7799749 I \\
\hline miR-68I8-5p & $0.04 \mid 32926$ & Up & 0.20318418 & 0.7799749 I \\
\hline miR-3926 & 0.04133196 & Up & 0.22292684 & $0.79|0224|$ \\
\hline miR-556-3p & 0.0413505 & Up & 0.54518596 & 0.92261663 \\
\hline miR-6506-3p & 0.04136633 & Up & 0.18207566 & 0.77344537 \\
\hline miR-5705 & $0.04|4| 633$ & Up & 0.20989329 & 0.78263372 \\
\hline miR-3605-5p & $0.04 \mid 47755$ & Up & 0.30032934 & 0.83872374 \\
\hline miR-92b-5p & 0.04159862 & Up & $0.0829376 I$ & 0.69839839 \\
\hline miR-5 I95-5p & 0.04165547 & Up & 0.16972762 & 0.77062416 \\
\hline miR-I250-3p & 0.04170601 & Up & 0.27258916 & 0.83107769 \\
\hline$m i R-49 I-5 p$ & 0.04204882 & Up & $0.097 \mid 4365$ & 0.7308563 I \\
\hline miR-5088-5p & 0.04206638 & $U_{p}$ & 0.44931883 & 0.88964976 \\
\hline miR-630 & 0.04207906 & Up & 0.31704859 & 0.84917098 \\
\hline miR-19a-3p & 0.04217759 & Up & 0.78509476 & 0.98667434 \\
\hline miR-3I54 & 0.04223574 & Up & 0.12130399 & 0.74226488 \\
\hline miR-68I3-3p & 0.04224463 & Up & 0.39270768 & 0.8730612 \\
\hline miR-3925-5p & 0.04246307 & $U_{p}$ & 0.27726756 & $0.83|0854|$ \\
\hline miR-193b-5p & $0.0425437 \mid$ & $U_{p}$ & 0.42377608 & 0.8823614 \\
\hline miR-3670 & 0.04282193 & $U_{p}$ & 0.11670866 & 0.74226488 \\
\hline miR-4453 & 0.04282594 & $U_{p}$ & 0.05055003 & 0.62458446 \\
\hline miR-427I & 0.0429164 & Up & $0.5525 \mid 367$ & 0.92384946 \\
\hline miR-6799-3p & 0.04300684 & Up & 0.07858756 & 0.68910078 \\
\hline miR-6753-3p & 0.04318932 & Up & 0.40952534 & 0.87779826 \\
\hline miR-4529-3p & 0.04328269 & $U_{p}$ & 0.16597797 & $0.7691508 \mid$ \\
\hline
\end{tabular}

(Continued) 
Table 9 (Continued).

\begin{tabular}{|c|c|c|c|c|}
\hline miRNAs & Log2FoldChange & Regulation & P-value & P-adj \\
\hline miR-6733-5p & 0.04335371 & $U_{p}$ & $0.2055966 \mid$ & 0.7804775 I \\
\hline miR-520f-5p & 0.04339194 & Up & 0.25109345 & $0.807|5| 25$ \\
\hline miR-4325 & 0.04340191 & Up & 0.15424249 & 0.75393664 \\
\hline miR-3667-5p & 0.04350045 & Up & 0.63222897 & 0.95096807 \\
\hline miR-3I86-3p & 0.0435136 & Up & $0.2353015 \mathrm{I}$ & 0.80202237 \\
\hline miR-650I-3p & 0.04367162 & Up & 0.09379904 & 0.72555728 \\
\hline miR-I 253 & 0.04375056 & Up & 0.1151096 & 0.74226488 \\
\hline miR-678I-3p & 0.04381511 & Up & 0.07438659 & 0.68406327 \\
\hline miR-5589-3p & 0.04405912 & Up & 0.2724389 & 0.83107769 \\
\hline miR-764 & 0.04417422 & Up & 0.11500808 & 0.74226488 \\
\hline miR-4262 & 0.04433845 & Up & 0.11386493 & 0.74226488 \\
\hline$m i R-68 \mid 4-3 p$ & $0.0443661 \mathrm{I}$ & Up & $0.2859361 \mathrm{I}$ & $0.8328976 \mathrm{I}$ \\
\hline miR-939-5p & 0.04444632 & Up & 0.61817978 & 0.94829596 \\
\hline miR-4648 & 0.04450342 & Up & $0.1454|4| 6$ & $0.7537626 \mathrm{I}$ \\
\hline miR-570I & 0.04456045 & Up & 0.73455339 & 0.97354829 \\
\hline miR-68।8-3p & 0.04464997 & Up & 0.21082098 & 0.78263372 \\
\hline miR-4777-5p & 0.0448246 & Up & 0.23921914 & 0.80380192 \\
\hline miR-597-5p & 0.04495088 & Up & 0.20999917 & 0.78263372 \\
\hline miR-4773 & $0.04495 \mid 22$ & Up & 0.17466302 & 0.77062416 \\
\hline miR-509-3-5p & 0.04497037 & Up & 0.13341303 & 0.74892227 \\
\hline miR-I539 & 0.04498088 & Up & $0.27863 \mid 26$ & $0.83|0854|$ \\
\hline miR-4733-3p & 0.04515748 & Up & 0.10700953 & 0.74226488 \\
\hline miR-I98 & 0.04517199 & Up & 0.1050005 & 0.74226488 \\
\hline miR-45I0 & 0.04528562 & $U_{p}$ & 0.11425075 & 0.74226488 \\
\hline$m i R-4743-3 p$ & $0.0454 I 78$ & Up & 0.06957496 & 0.67187824 \\
\hline miR-548az-3p & 0.04548397 & Up & 0.16890955 & 0.77062416 \\
\hline miR-7843-5p & 0.04554652 & Up & 0.06781769 & 0.66270515 \\
\hline miR-4672 & 0.045715 & Up & 0.6358909 & 0.95096807 \\
\hline miR-4486 & $0.04580 \mid 43$ & Up & 0.22915805 & $0.79 \mid 58088$ \\
\hline miR-4725-3p & 0.04588192 & $U_{p}$ & 0.09281998 & $0.7228707 \mid$ \\
\hline miR-3I76 & 0.04606993 & Up & 0.2120879 & 0.78263372 \\
\hline miR-6894-3p & 0.04622563 & Up & 0.3885454 & 0.87210628 \\
\hline miR-3I37 & 0.04657398 & Up & 0.36426293 & 0.8588192 \\
\hline miR-6893-3p & 0.0466731 & Up & $0.3260665 I$ & 0.85489649 \\
\hline miR-6780a-3p & 0.04669084 & Up & 0.39101807 & $0.8728 \mid 803$ \\
\hline miR-5696 & 0.04708647 & Up & $0.28288 \mid 92$ & 0.83193599 \\
\hline miR-296-5p & 0.04722118 & Up & 0.37763845 & 0.8649907 I \\
\hline miR-65I6-3p & $0.04725 \mid 37$ & Up & 0.29769649 & 0.83872374 \\
\hline$m i R-3613-3 p$ & 0.04725775 & Up & 0.24707509 & $0.807|5| 25$ \\
\hline miR-8053 & 0.0473133 & Up & 0.20270231 & 0.7799749 I \\
\hline miR-3I52-5p & 0.04732823 & Up & 0.19598155 & 0.7799749 I \\
\hline miR-57I & 0.04734154 & Up & 0.84533752 & $0.9965428 I$ \\
\hline $\mathrm{miR}-378 \mathrm{~b}$ & 0.04739432 & Up & 0.06860943 & 0.66790237 \\
\hline miR-499b-5p & 0.04800234 & Up & 0.27973448 & $0.83|0854|$ \\
\hline miR-I47a & $0.048 \mid 8405$ & Up & $0.066 \mid 8544$ & 0.65890175 \\
\hline miR-892c-3p & 0.04821604 & Up & 0.27352161 & 0.83107769 \\
\hline$m i R-6762-3 p$ & 0.04841079 & Up & 0.08410509 & 0.70009532 \\
\hline miR-6747-5p & 0.04842416 & Up & 0.05004196 & 0.62129395 \\
\hline miR-4523 & 0.04855634 & Up & 0.0620686 & 0.65108692 \\
\hline
\end{tabular}

(Continued) 
Table 9 (Continued).

\begin{tabular}{|c|c|c|c|c|}
\hline miRNAs & Log2FoldChange & Regulation & P-value & P-adj \\
\hline miR-2I5-3p & 0.04857459 & Up & 0.35706226 & 0.8588192 \\
\hline miR-3675-5p & 0.04879536 & Up & 0.25507552 & 0.81031406 \\
\hline$m i R-488-5 p$ & 0.04908388 & Up & 0.08417489 & 0.70009532 \\
\hline miR-548au-5p & 0.04911752 & Up & $0.083|556|$ & 0.69839839 \\
\hline miR-I827 & 0.04916334 & Up & 0.04790815 & $0.614|250|$ \\
\hline miR-4475 & 0.04922564 & Up & 0.24552818 & $0.807|5| 25$ \\
\hline $\mathrm{miR}-4538$ & 0.04929697 & Up & 0.15188194 & 0.75393664 \\
\hline$m i R-4524 a-3 p$ & 0.04946585 & Up & 0.16715218 & 0.76915081 \\
\hline miR-I 297 & 0.04951592 & Up & 0.13022476 & 0.74892227 \\
\hline miR-545-5p & 0.0495214 & Up & 0.21665067 & 0.7853205 \\
\hline miR-6848-3p & 0.0496268 & Up & 0.25624536 & 0.81102287 \\
\hline miR-592 & 0.04984516 & Up & 0.24492201 & 0.80715125 \\
\hline miR-4762-3p & $0.0499 \mid 254$ & Up & 0.19551925 & 0.77997491 \\
\hline miR-3I57-3p & 0.05023291 & Up & 0.06067089 & 0.64832335 \\
\hline miR-424-3p & 0.05037082 & Up & 0.13197357 & 0.74892227 \\
\hline miR-3180-5p & 0.05065927 & $U_{p}$ & 0.21258503 & 0.78263372 \\
\hline miR-432I & 0.05096856 & Up & 0.12083398 & 0.74226488 \\
\hline miR-564 & 0.05101648 & Up & 0.67354792 & 0.95648223 \\
\hline miR-7I55-5p & 0.05113808 & $U_{p}$ & 0.06047598 & 0.64832335 \\
\hline $\mathrm{miR}-4460$ & 0.05115466 & Up & 0.12549433 & 0.74465526 \\
\hline miR-4539 & 0.05115494 & Up & 0.0665464 & 0.65890175 \\
\hline miR-4793-5p & 0.05132612 & Up & 0.2768576 & $0.83|0854|$ \\
\hline miR-452I & 0.05141996 & $U_{p}$ & 0.17417833 & 0.77062416 \\
\hline miR-3I35b & $0.0517 \mid 743$ & $U_{p}$ & 0.67625049 & 0.95648223 \\
\hline miR-6793-3p & 0.05175787 & Up & 0.12086294 & 0.74226488 \\
\hline miR-3।43 & 0.05205933 & Up & 0.22745616 & $0.79|0224|$ \\
\hline $\mathrm{miR}-1273 \mathrm{f}$ & 0.05237566 & $U_{p}$ & 0.3795035 & | 0.8649907 \\
\hline miR-297 & $0.0524|93|$ & $U_{p}$ & 0.12491919 & 0.74465526 \\
\hline miR-4675 & 0.05253927 & Up & 0.14239401 & 0.75376261 \\
\hline miR-4488 & 0.05266286 & $U_{p}$ & 0.176305 & 0.77062416 \\
\hline miR-4489 & 0.05272056 & $U_{p}$ & 0.1245739 & 0.74454632 \\
\hline miR-6757-3p & 0.05273464 & Up & $0.4352728 I$ & 0.88941554 \\
\hline miR-569 & 0.0527518 & Up & 0.15673419 & $0.757 \mid 15578$ \\
\hline miR-2II 3 & $0.05283 \mid 36$ & Up & 0.18308273 & $0.7735088 \mathrm{I}$ \\
\hline miR-4696 & 0.05289934 & Up & 0.06296921 & 0.65539395 \\
\hline miR-216a-3p & 0.05300228 & $U_{p}$ & 0.11694725 & 0.74226488 \\
\hline miR-8069 & 0.05327677 & $U_{p}$ & 0.74358134 & 0.97549977 \\
\hline miR-378f & 0.05332519 & $U_{p}$ & 0.33432056 & 0.85489649 \\
\hline miR-562 & 0.05334213 & Up & 0.20140266 & | 0.7799749 \\
\hline miR-6732-3p & 0.05336692 & Up & 0.2016338 & | 0.7799749 \\
\hline miR-6879-3p & 0.05340118 & Up & 0.22409885 & $0.79|0224|$ \\
\hline miR-135b-5p & 0.05351659 & $U_{p}$ & 0.20225766 & | 0.7799749 \\
\hline miR-589-5p & $0.0537955 I$ & Up & 0.08919464 & $0.7|4| I 286$ \\
\hline miR-548m & 0.05393355 & Up & 0.35511374 & 0.8588192 \\
\hline$m i R-4316$ & 0.05396877 & $U_{p}$ & 0.13140033 & 0.74892227 \\
\hline miR-7/08-5p & $0.0542 I 148$ & Up & 0.58185476 & 0.93436563 \\
\hline miR-4758-3p & 0.05427522 & Up & 0.40499182 & 0.87475982 \\
\hline miR-3974 & 0.05440058 & Up & $0.03934 \mid 44$ & $0.5987 \mid 978$ \\
\hline miR-6499-3p & 0.05455931 & $U_{p}$ & 0.19238152 & | 0.7799749 \\
\hline
\end{tabular}

(Continued) 
Table 9 (Continued).

\begin{tabular}{|c|c|c|c|c|}
\hline miRNAs & Log2FoldChange & Regulation & P-value & P-adj \\
\hline miR-7I56-3p & 0.05474298 & $U_{p}$ & 0.11579842 & 0.74226488 \\
\hline miR-3677-5p & 0.05477731 & Up & 0.11357964 & 0.74226488 \\
\hline miR-4802-5p & 0.05510145 & Up & 0.12191501 & 0.74423174 \\
\hline$m i R-5 \mid 2-3 p$ & 0.05528425 & Up & $0.2807|56|$ & $0.83|0854|$ \\
\hline$m i R-548 g-3 p$ & 0.05529729 & Up & $0.4257 \mid 423$ & 0.8823614 \\
\hline miR-4768-5p & 0.05542136 & Up & 0.25826154 & 0.81339724 \\
\hline miR-548ad-3p & 0.05542262 & Up & 0.18370678 & $0.7735088 \mathrm{I}$ \\
\hline miR-4795-3p & 0.05570005 & Up & 0.11924634 & 0.74226488 \\
\hline miR-7III-5p & $0.0558|37|$ & Up & 0.04063447 & 0.60118096 \\
\hline miR-I469 & 0.05624999 & Up & 0.09534062 & 0.72924227 \\
\hline miR-3652 & 0.0564083 & Up & 0.2054363 & 0.7804775 I \\
\hline miR-933 & 0.05650599 & Up & 0.12011652 & 0.74226488 \\
\hline miR-3II7-3p & 0.05656766 & Up & 0.12111369 & 0.74226488 \\
\hline miR-3190-5p & 0.05684382 & Up & $0.2286 \mid 872$ & $0.79|0224|$ \\
\hline miR-676I-3p & 0.05710884 & Up & 0.16210545 & 0.76582906 \\
\hline miR-6835-3p & 0.05736794 & Up & 0.27628877 & $0.83|0854|$ \\
\hline miR-6080 & 0.05767708 & Up & 0.17847595 & 0.77062416 \\
\hline $\mathrm{miR}-4300$ & 0.05768393 & Up & 0.05365624 & 0.63883244 \\
\hline miR-4429 & 0.0577899 & Up & 0.04674782 & $0.6|4| 250 \mid$ \\
\hline miR-I 270 & 0.05788983 & Up & $0.01208 \mid 24$ & 0.44355407 \\
\hline miR-526b-5p & 0.05789863 & Up & 0.08022807 & 0.68958576 \\
\hline miR-4639-5p & 0.05791153 & Up & 0.14529474 & $0.7537626 \mathrm{I}$ \\
\hline miR-5588-5p & 0.05792606 & Up & $0.0358 \mid 121$ & 0.59871978 \\
\hline$m i R-615-5 p$ & 0.05796346 & $U_{p}$ & 0.1150281 & 0.74226488 \\
\hline miR-I249-5p & 0.05819312 & Up & 0.3756047 I & 0.8649907 I \\
\hline miR-1252-5p & 0.05822212 & Up & 0.10268676 & 0.73922964 \\
\hline miR-150-3p & 0.05825973 & Up & $0.2592485 I$ & 0.81550632 \\
\hline miR-4790-5p & 0.05830035 & Up & 0.10614619 & 0.74226488 \\
\hline miR-3678-5p & $0.0586658 I$ & Up & 0.30176797 & 0.83872374 \\
\hline miR-222-3p & 0.05867964 & $U_{p}$ & $0.3181913 \mid$ & 0.84917098 \\
\hline miR-4786-5p & 0.0587769 & Up & $0.088 \mid 7894$ & $0.7109058 \mathrm{I}$ \\
\hline miR-68I2-5p & 0.05917651 & Up & 0.56525274 & 0.9303065 \\
\hline$m i R-3 \mid 45-3 p$ & $0.0592808 I$ & Up & 0.17130088 & 0.77062416 \\
\hline miR-60I & 0.05930908 & Up & 0.11511752 & 0.74226488 \\
\hline miR-450a-2-3p & 0.0594454 & Up & 0.1464859 & $0.7537626 \mathrm{I}$ \\
\hline miR-32-3p & 0.05951562 & Up & 0.14474059 & $0.7537626 \mathrm{I}$ \\
\hline miR-1908-3p & $0.0596|36|$ & Up & 0.43830338 & 0.88952595 \\
\hline miR-3162-5p & 0.05982246 & Up & 0.45070972 & 0.88964976 \\
\hline miR-664b-3p & 0.05992559 & Up & 0.79741636 & 0.98759926 \\
\hline miR-3922-3p & 0.0599527 & Up & 0.15889256 & 0.75988515 \\
\hline miR-4458 & 0.06005394 & Up & 0.09890351 & 0.7308563 I \\
\hline miR-6826-3p & 0.06023102 & Up & 0.13036518 & 0.74892227 \\
\hline miR-3605-3p & 0.06051898 & Up & 0.16045339 & $0.76082 \mid 42$ \\
\hline miR-548al & $0.0605274 \mid$ & Up & 0.10733331 & 0.74226488 \\
\hline miR-30la-5p & $0.0605335 I$ & Up & 0.16686685 & $0.769|508|$ \\
\hline miR-566 & 0.06055698 & Up & 0.110184 & 0.74226488 \\
\hline$m i R-6775-3 p$ & 0.06081053 & Up & 0.32525563 & 0.85489649 \\
\hline$m i R-24-2-5 p$ & 0.06086829 & $U_{p}$ & 0.27107456 & 0.83107769 \\
\hline$m i R-4745-5 p$ & $0.06148 \mid 74$ & Up & 0.26320069 & $0.82|9728|$ \\
\hline
\end{tabular}

(Continued) 
Table 9 (Continued).

\begin{tabular}{|c|c|c|c|c|}
\hline miRNAs & Log2FoldChange & Regulation & P-value & P-adj \\
\hline miR-I42-5p & 0.0615305 & Up & 0.74062343 & 0.97402999 \\
\hline$m i R-3 \mid 45-5 p$ & 0.06155958 & Up & $0.2705365 I$ & 0.83107769 \\
\hline$m i R-619-3 p$ & 0.06191006 & Up & 0.04090207 & 0.60118096 \\
\hline miR-4697-3p & 0.06193714 & Up & 0.03581024 & $0.5987 \mid 978$ \\
\hline miR-1913 & 0.06196023 & Up & 0.14965706 & 0.75393664 \\
\hline miR-I227-3p & 0.0624576 & Up & 0.26520529 & 0.82438552 \\
\hline miR-6857-3p & 0.06249059 & Up & 0.23882079 & 0.80380192 \\
\hline miR-6789-3p & 0.0627565 & Up & 0.0762451 & 0.68910078 \\
\hline miR-520a-3p & $0.0633444 I$ & Up & 0.24890997 & 0.80715125 \\
\hline miR-7977 & 0.06345694 & Up & 0.74952299 & 0.97742837 \\
\hline miR-3622b-5p & 0.06355498 & Up & $0.046 \mid 498$ & $0.6|4| 250 \mid$ \\
\hline miR-1273h-3p & $0.0636431 \mathrm{I}$ & Up & 0.0940119 & 0.72555728 \\
\hline miR-I305 & $0.06377 \mid 45$ & $U_{p}$ & 0.32183809 & 0.85270505 \\
\hline miR-8089 & 0.06424523 & Up & 0.17909857 & 0.77062416 \\
\hline miR-4663 & 0.06434253 & Up & 0.02836095 & 0.59258245 \\
\hline miR-47I5-5p & $0.0644967 \mid$ & Up & 0.03872527 & $0.5987 / 978$ \\
\hline$m i R-6508-5 p$ & $0.06483 \mid 37$ & Up & 0.51403325 & 0.91376348 \\
\hline miR-3935 & 0.06495576 & Up & 0.33347485 & 0.85489649 \\
\hline miR-6867-3p & 0.06507568 & Up & 0.04850407 & $0.614|250|$ \\
\hline miR-492 & $0.065|685|$ & Up & 0.04991087 & 0.62129395 \\
\hline miR-5094 & 0.06523354 & Up & 0.06079608 & 0.64832335 \\
\hline miR-6865-3p & 0.06624252 & Up & 0.21168754 & 0.78263372 \\
\hline miR-4493 & 0.06642774 & $U_{p}$ & 0.0392186 & 0.59871978 \\
\hline miR-7II4-3p & 0.06671008 & $U_{p}$ & 0.13302163 & 0.74892227 \\
\hline miR-4288 & 0.06683348 & Up & 0.0299282 & 0.59616058 \\
\hline let-7i-3p & 0.06709042 & Up & 0.30201312 & 0.83872374 \\
\hline miR-3975 & 0.06765074 & Up & 0.11109946 & 0.74226488 \\
\hline$m i R-3 \mid 20-5 p$ & 0.0678456 & Up & 0.07987684 & 0.68910078 \\
\hline miR-7II4-5p & 0.06800749 & Up & 0.6569214 & 0.95321673 \\
\hline miR-548ah-5p & 0.06819495 & $U_{p}$ & 0.07413876 & 0.68406327 \\
\hline$m i R-5 \mid 3 c-5 p$ & 0.06843293 & $U_{p}$ & 0.40487621 & 0.87475982 \\
\hline let-7f-2-3p & 0.06854085 & Up & 0.18035401 & 0.77062416 \\
\hline miR-47।6-5p & 0.06872344 & Up & 0.20833589 & 0.78263372 \\
\hline miR-4787-3p & 0.06876198 & Up & 0.50943723 & 0.91230009 \\
\hline miR-600 & 0.06877777 & Up & 0.07989689 & 0.68910078 \\
\hline miR-7I50 & 0.06879546 & Up & 0.26528174 & 0.82438552 \\
\hline miR-29a-5p & 0.06883865 & $U_{p}$ & 0.14662388 & 0.75376261 \\
\hline miR-I84 & 0.06888405 & Up & 0.01862817 & 0.53734625 \\
\hline miR-6870-3p & 0.06911219 & Up & 0.23957676 & 0.80380192 \\
\hline miR-6850-3p & 0.06919394 & Up & 0.25777855 & 0.81287224 \\
\hline miR-4657 & 0.06968547 & Up & 0.12522401 & 0.74465526 \\
\hline miR-37lb-5p & 0.06969313 & Up & 0.23932344 & 0.80380192 \\
\hline$m i R-4680-5 p$ & 0.06974422 & Up & $0.0340474 \mid$ & $0.5987 \mid 978$ \\
\hline miR-6499-5p & 0.07006264 & Up & 0.04920309 & 0.61683874 \\
\hline miR-68I0-3p & 0.0701419 & Up & 0.06035347 & 0.64832335 \\
\hline miR-3682-3p & 0.07039938 & $U_{p}$ & 0.17881596 & 0.77062416 \\
\hline miR-6I 26 & 0.0704907 & Up & 0.28825395 & 0.83439474 \\
\hline$m i R-653-5 p$ & 0.07088698 & Up & 0.03087676 & $0.5978|47|$ \\
\hline miR-382-3p & 0.07116744 & $U_{p}$ & 0.18031035 & 0.77062416 \\
\hline
\end{tabular}

(Continued) 
Table 9 (Continued).

\begin{tabular}{|c|c|c|c|c|}
\hline miRNAs & Log2FoldChange & Regulation & P-value & P-adj \\
\hline miR-6858-3p & 0.07117186 & $U_{p}$ & 0.38553306 & 0.86961208 \\
\hline miR-4499 & $0.07 / 22958$ & Up & $0.2484 \mid I 2 I$ & $0.807|5| 25$ \\
\hline miR-5I87-3p & 0.07144019 & Up & $0.023 \mid 2432$ & 0.53734625 \\
\hline miR-557 & 0.07164553 & Up & 0.04546064 & $0.6141250 \mid$ \\
\hline miR-I28I & 0.07198433 & Up & 0.40118929 & 0.87364718 \\
\hline miR-6786-5p & 0.07206845 & Up & 0.43792054 & 0.88952595 \\
\hline miR-455-3p & 0.07223937 & Up & 0.12343738 & 0.74454632 \\
\hline miR-5I8d-3p & 0.07256516 & Up & 0.18334791 & $0.7735088 I$ \\
\hline miR-286I & 0.07268732 & Up & $0.356 \mid 5086$ & 0.8588192 \\
\hline miR-648 & 0.07270619 & Up & 0.03799809 & 0.59871978 \\
\hline$m i R-5 I 5-3 p$ & 0.07293229 & Up & 0.08596047 & 0.70807179 \\
\hline miR-4329 & 0.07336799 & Up & 0.07363192 & 0.68406327 \\
\hline miR-4652-5p & $0.07337|9|$ & Up & 0.02576632 & $0.55959 \mid 48$ \\
\hline miR-935 & 0.0735817 & Up & 0.18143568 & 0.77328308 \\
\hline miR-4738-5p & 0.07367585 & Up & 0.01998951 & 0.53734625 \\
\hline miR-6784-3p & 0.07382632 & Up & 0.03657297 & 0.59871978 \\
\hline miR-500a-5p & 0.07386426 & Up & 0.15142982 & 0.75393664 \\
\hline miR-2053 & 0.07413253 & Up & 0.1346475 & 0.75226974 \\
\hline $\mathrm{miR}-45 \mathrm{Ib}$ & 0.07445664 & Up & $0.0637571 I$ & $0.655423 \mathrm{II}$ \\
\hline miR-6800-3p & 0.07499934 & Up & 0.1424747 & 0.75376261 \\
\hline miR-558I-5p & 0.07511844 & Up & 0.21009407 & 0.78263372 \\
\hline miR-642b-3p & 0.07598197 & Up & 0.36915419 & 0.86195734 \\
\hline miR-37la-3p & 0.07664063 & Up & $0.087 \mid 1322$ & 0.70907074 \\
\hline miR-548ae-3p & 0.07679238 & $U_{p}$ & 0.21885155 & 0.78840757 \\
\hline miR-4753-3p & 0.077048 & Up & 0.00651694 & 0.40231598 \\
\hline miR-4653-3p & $0.077494 I I$ & Up & 0.12336065 & 0.74454632 \\
\hline miR-550a-5p & 0.07848802 & Up & $0.2479 \mid 286$ & $0.807 / 5125$ \\
\hline miR-509-3p & 0.07856046 & Up & 0.03093376 & $0.5978|47|$ \\
\hline miR-3607-3p & $0.0787|8| 4$ & Up & 0.6208883 & 0.94829596 \\
\hline miR-3620-3p & 0.07893846 & $U_{p}$ & 0.04506553 & $0.614|250|$ \\
\hline miR-3943 & 0.07894053 & Up & 0.02604419 & 0.55959148 \\
\hline miR-6502-5p & 0.07908038 & Up & 0.20157974 & $0.7799749 \mid$ \\
\hline$m i R-3|5|-3 p$ & 0.07967298 & Up & 0.12119654 & 0.74226488 \\
\hline miR-I255a & 0.07973658 & Up & 0.05181195 & 0.63253996 \\
\hline$m i R-34 c-3 p$ & 0.07994349 & Up & 0.09180651 & 0.72153737 \\
\hline miR-I252-3p & 0.08011389 & Up & 0.03880862 & 0.59871978 \\
\hline miR-186-3p & 0.08026808 & Up & 0.03610748 & 0.59871978 \\
\hline$m i R-689 \mid-5 p$ & 0.08058981 & Up & 0.28582416 & 0.83289761 \\
\hline miR-3646 & $0.08099 \mid 4$ & Up & $0.4 \mid 406383$ & 0.8804535 \\
\hline miR-7II0-5p & $0.08|43| 44$ & Up & 0.17902444 & 0.77062416 \\
\hline miR-6824-3p & 0.08146995 & Up & 0.12843883 & 0.74892227 \\
\hline miR-135a-3p & 0.08192344 & Up & 0.26560242 & 0.82438552 \\
\hline miR-I44-3p & 0.08254493 & Up & 0.81458209 & 0.99122915 \\
\hline miR-3I95 & 0.08270457 & Up & 0.24420862 & $0.807|5| 25$ \\
\hline miR-548az-5p & $0.0830347 \mid$ & Up & 0.0395986 & 0.59871978 \\
\hline miR-3945 & 0.08394863 & Up & 0.12433998 & 0.74454632 \\
\hline miR-3I39 & 0.08450308 & Up & 0.02320834 & 0.53734625 \\
\hline miR-679I-5p & 0.08464489 & $U_{p}$ & 0.4183558 & 0.88201345 \\
\hline miR-1915-3p & 0.08517208 & Up & 0.53746958 & 0.9213181 \\
\hline
\end{tabular}

(Continued) 
Table 9 (Continued).

\begin{tabular}{|c|c|c|c|c|}
\hline miRNAs & Log2FoldChange & Regulation & P-value & P-adj \\
\hline miR-542-5p & 0.08526668 & Up & 0.46783284 & 0.89396011 \\
\hline$m i R-519 e-3 p$ & $0.085674 \mid 8$ & Up & $0.1175949 \mid$ & 0.74226488 \\
\hline miR-5585-3p & 0.08579912 & Up & $0.4617884 \mid$ & 0.89396011 \\
\hline miR-378g & 0.08622678 & Up & 0.05316056 & 0.63842356 \\
\hline$m i R-548 h-5 p$ & $0.0864|97|$ & Up & 0.18294233 & $0.7735088 \mathrm{I}$ \\
\hline miR-6895-3p & 0.08645486 & Up & 0.02529787 & 0.55568816 \\
\hline miR-8060 & 0.08676058 & Up & 0.09109285 & 0.72012996 \\
\hline miR-6738-3p & $0.08676 \mid 32$ & Up & 0.07990351 & 0.68910078 \\
\hline miR-806I & 0.08705854 & Up & 0.01130452 & 0.43937422 \\
\hline miR-7I62-3p & 0.08711663 & Up & 0.02294984 & 0.53734625 \\
\hline miR-6737-3p & $0.08722 \mid 44$ & Up & 0.25604062 & 0.81102287 \\
\hline miR-6867-5p & $0.08729|8|$ & Up & 0.05940578 & 0.64832335 \\
\hline miR-I267 & $0.08783 \mid 25$ & $U_{p}$ & 0.01084837 & 0.43937422 \\
\hline miR-359I-3p & 0.08842998 & Up & 0.00854575 & 0.40696367 \\
\hline$m i R-65 I 5-5 p$ & $0.0889700 \mathrm{I}$ & Up & 0.04215507 & 0.60118096 \\
\hline let-7c-5p & 0.08921252 & Up & 0.50684609 & 0.912181 \\
\hline miR-377-3p & 0.08929848 & Up & 0.61601294 & 0.94741169 \\
\hline miR-602 & 0.08990339 & Up & 0.08499877 & $0.70240|4|$ \\
\hline miR-469I-5p & $0.0923398 I$ & Up & 0.06685589 & 0.65890175 \\
\hline miR-876-3p & 0.09288635 & Up & 0.04792887 & $0.614 \mid 2501$ \\
\hline$m i R-23 b-5 p$ & 0.0930458 & Up & 0.03197789 & $0.5987 / 978$ \\
\hline miR-548am-3p & 0.09494395 & Up & 0.00255307 & 0.34045223 \\
\hline miR-96-5p & 0.09510759 & $U_{p}$ & 0.13818989 & 0.75376261 \\
\hline miR-6I4 & 0.0955557 I & $U_{p}$ & 0.02218303 & 0.53734625 \\
\hline miR-29a-3p & $0.0957077 \mid$ & Up & 0.31794301 & 0.84917098 \\
\hline$m i R-532-5 p$ & 0.09574706 & Up & 0.53567007 & 0.9213181 \\
\hline miR-135a-5p & $0.0965465 I$ & Up & 0.0829293 & 0.69839839 \\
\hline miR-193a-5p & 0.09719116 & Up & 0.09703079 & 0.73085631 \\
\hline miR-4497 & 0.09803222 & Up & 0.17758974 & 0.77062416 \\
\hline miR-4433a-5p & 0.09851921 & $U_{p}$ & 0.03675037 & $0.5987 \mid 978$ \\
\hline miR-1277-3p & 0.09875703 & $U_{p}$ & 0.03483406 & $0.5987 \mid 978$ \\
\hline miR-6507-3p & 0.09886526 & Up & 0.09969864 & 0.73085631 \\
\hline miR-890 & $0.099524 \mid 4$ & Up & 0.0034835 & 0.34045223 \\
\hline miR-1234-3p & 0.09955079 & Up & 0.36352269 & 0.8588192 \\
\hline$m i R-685 I-3 p$ & 0.09975676 & Up & 0.19181651 & 0.77997491 \\
\hline miR-8084 & 0.09976723 & Up & 0.00359751 & 0.34045223 \\
\hline$m i R-4725-5 p$ & $0.0997807 \mid$ & Up & 0.21267843 & 0.78263372 \\
\hline miR-3I55b & $0.09979 \mid 22$ & Up & 0.00278283 & 0.34045223 \\
\hline miR-50I-5p & $0.1003276 \mathrm{I}$ & Up & 0.39061255 & $0.8728 \mid 803$ \\
\hline miR-4507 & 0.10099259 & Up & 0.31915037 & 0.84972893 \\
\hline$m i R-18 I b-5 p$ & 0.10183856 & Up & 0.35637515 & 0.8588192 \\
\hline$m i R-4800-5 p$ & 0.10217379 & Up & 0.22684006 & 0.79102241 \\
\hline miR-I973 & 0.10318636 & Up & 0.27355317 & 0.83107769 \\
\hline miR-876-5p & 0.10322575 & Up & 0.00648836 & 0.40231598 \\
\hline miR-4689 & 0.10347357 & Up & 0.15000646 & 0.75393664 \\
\hline$m i R-4485-5 p$ & $0.103767 / 2$ & Up & 0.42637587 & 0.88249386 \\
\hline miR-I 470 & 0.1042914 & Up & 0.14840618 & 0.75376261 \\
\hline$m i R-4536-3 p$ & 0.10440502 & Up & $0.007975 \mid 2$ & 0.40696367 \\
\hline miR-4478 & 0.10495 & $U_{p}$ & 0.0747843 & 0.68406327 \\
\hline
\end{tabular}

(Continued) 
Table 9 (Continued).

\begin{tabular}{|c|c|c|c|c|}
\hline miRNAs & Log2FoldChange & Regulation & P-value & P-adj \\
\hline miR-43I0 & 0.1058934 & $U_{p}$ & 0.10095486 & 0.73085631 \\
\hline miR-98-3p & 0.10626554 & Up & 0.02112264 & 0.53734625 \\
\hline miR-575 & 0.1064784 & Up & 0.35501009 & 0.8588192 \\
\hline miR-I5a-5p & 0.10652983 & Up & 0.35983269 & 0.8588192 \\
\hline miR-6858-5p & 0.10799872 & Up & 0.14672844 & 0.75376261 \\
\hline miR-224-3p & 0.10808806 & Up & 0.11591039 & 0.74226488 \\
\hline miR-3II 8 & 0.10852694 & Up & 0.0138673 & 0.46600109 \\
\hline miR-660-5p & 0.10883293 & Up & 0.46688213 & 0.89396011 \\
\hline miR-663a & 0.10974097 & Up & 0.28324328 & 0.83193599 \\
\hline miR-I238-5p & 0.11034519 & Up & 0.01534139 & 0.49284222 \\
\hline miR-4665-3p & 0.11195401 & Up & 0.49477477 & 0.90848159 \\
\hline miR-7704 & 0.11225656 & Up & 0.09124377 & 0.72012996 \\
\hline miR-43I2 & 0.1126684 & Up & 0.06065803 & 0.64832335 \\
\hline miR-4466 & 0.1128636 & Up & 0.44582227 & 0.88952595 \\
\hline miR-3074-3p & 0.1131561 & Up & 0.01597018 & 0.50670832 \\
\hline miR-6I31 & 0.11373774 & Up & 0.11445403 & 0.74226488 \\
\hline miR-394I & 0.1148302 & Up & 0.00122462 & 0.26227331 \\
\hline miR-4449 & 0.11599411 & Up & 0.33275425 & 0.85489649 \\
\hline$m i R-342-5 p$ & 0.11633261 & Up & 0.46550933 & $0.893960 \mathrm{II}$ \\
\hline$m i R-548 c-3 p$ & 0.11721243 & Up & 0.0425739 & 0.60118096 \\
\hline miR-638 & 0.11790044 & Up & 0.10193756 & 0.73589757 \\
\hline miR-937-5p & 0.11821584 & Up & 0.16926304 & 0.77062416 \\
\hline miR-6769b-3p & 0.1190295 & Up & $0.0006 \mid 825$ & 0.15302397 \\
\hline$m i R-483-3 p$ & 0.12066504 & $U_{p}$ & $0.06664 \mid 88$ & 0.65890175 \\
\hline miR-6752-3p & 0.1209839 & Up & $0.1174648 \mid$ & 0.74226488 \\
\hline miR-3I64 & 0.12126773 & Up & 0.02485249 & 0.55568816 \\
\hline miR-425-3p & 0.12135184 & Up & 0.07479447 & 0.68406327 \\
\hline$m i R-65 I 5-3 p$ & 0.12361515 & Up & $0.2989434 \mid$ & 0.83872374 \\
\hline miR-3I 25 & 0.12402533 & Up & $0.1545 \mid 361$ & 0.75393664 \\
\hline miR-I825 & 0.12797268 & $U_{p}$ & 0.05740226 & 0.64832335 \\
\hline miR-6797-3p & 0.13035638 & Up & 0.16601533 & 0.76915081 \\
\hline miR-II8I & 0.13038863 & Up & $0.1207|58|$ & 0.74226488 \\
\hline miR-682I-5p & 0.13109406 & Up & 0.37417957 & 0.8649907 I \\
\hline miR-I238-3p & 0.13295731 & Up & 0.08977201 & $0.7 \mid 650331$ \\
\hline miR-6890-3p & 0.13318201 & Up & 0.00293306 & 0.34045223 \\
\hline miR-I260a & 0.13337208 & Up & 0.32936032 & 0.85489649 \\
\hline miR-8072 & 0.13401883 & Up & 0.00902604 & 0.40696367 \\
\hline miR-6880-3p & 0.13423449 & Up & 0.01863114 & 0.53734625 \\
\hline miR-6734-5p & 0.13476722 & Up & 0.02094573 & 0.53734625 \\
\hline miR-5580-3p & 0.13672314 & Up & 0.05083706 & $0.625 I 2553$ \\
\hline miR-I 304-3p & 0.1371615 & Up & 0.00400137 & 0.34045223 \\
\hline miR-199b-5p & 0.13739765 & Up & 0.5988803 & 0.93906184 \\
\hline miR-4465 & 0.13988188 & Up & $0.355 \mid 3979$ & 0.8588192 \\
\hline miR-4746-3p & 0.14518648 & Up & 0.02208845 & 0.53734625 \\
\hline miR-6759-3p & 0.14532219 & Up & $0.083 \mid 3556$ & 0.69839839 \\
\hline let-7b-5p & 0.14614295 & Up & $0.3508 I 789$ & 0.8588192 \\
\hline miR-4634 & 0.14754824 & Up & 0.14617632 & 0.75376261 \\
\hline miR-4505 & 0.14761153 & Up & 0.04246345 & 0.60118096 \\
\hline miR-502-3p & $0.1478068 \mid$ & Up & 0.1566901 & 0.75715578 \\
\hline
\end{tabular}

(Continued) 
Table 9 (Continued).

\begin{tabular}{|c|c|c|c|c|}
\hline miRNAs & Log2FoldChange & Regulation & P-value & P-adj \\
\hline miR-4649-3p & 0.14788706 & Up & 0.01102337 & 0.43937422 \\
\hline $\operatorname{miR}-6 \mid 24$ & 0.15010733 & Up & 0.20156221 & 0.77997491 \\
\hline miR-4687-3p & 0.15063161 & Up & 0.33744806 & 0.85489649 \\
\hline let-7f-I-3p & 0.15074119 & Up & 0.05525242 & 0.64056718 \\
\hline miR-636 & 0.15213534 & Up & 0.00198209 & 0.32405862 \\
\hline miR-762 & 0.15570984 & $U_{p}$ & 0.08053749 & 0.68993782 \\
\hline miR-I228-3p & 0.15582107 & $U_{p}$ & 0.16159926 & 0.76484365 \\
\hline let-7i-5p & 0.1564672 & Up & 0.04506864 & $0.614|250|$ \\
\hline$m i R-6803-5 p$ & 0.15706346 & Up & 0.31237204 & 0.8475125 \\
\hline$m i R-6893-5 p$ & 0.15994903 & Up & 0.11245159 & 0.74226488 \\
\hline miR-33b-3p & 0.16368374 & $U_{p}$ & 0.15801165 & 0.75988515 \\
\hline$m i R-34 b-5 p$ & 0.16407042 & Up & 0.04167753 & 0.60118096 \\
\hline$m i R-5 \mid 3 b-5 p$ & 0.16733719 & $U_{p}$ & 0.14386933 & 0.75376261 \\
\hline miR-3665 & 0.16739733 & $U_{p}$ & 0.40603336 & 0.87475982 \\
\hline $\operatorname{miR}-4785$ & 0.16909089 & $U_{p}$ & 0.01306086 & 0.45981375 \\
\hline miR-140-3p & 0.17052129 & $U_{p}$ & 0.039837 & 0.59871978 \\
\hline miR-3196 & 0.17113537 & $U_{p}$ & $0.2162073 \mid$ & 0.7853205 \\
\hline miR-19I-3p & 0.17179396 & Up & 0.04487927 & 0.61412501 \\
\hline miR-45I5 & 0.17261046 & $U_{p}$ & 0.01939174 & 0.53734625 \\
\hline$m i R-125 b-5 p$ & 0.17326977 & $U_{p}$ & 0.58039482 & 0.93436563 \\
\hline miR-4284 & $0.1749924 I$ & $U_{p}$ & 0.13111512 & 0.74892227 \\
\hline miR-67I7-5p & 0.17647607 & $U_{p}$ & 0.07006357 & 0.67187824 \\
\hline miR-4455 & 0.17661528 & $U_{p}$ & 0.05721903 & 0.64832335 \\
\hline miR-4767 & 0.17813392 & $U_{p}$ & 0.00704444 & 0.40231598 \\
\hline miR-7107-5p & 0.17892282 & $U_{p}$ & 0.25114209 & $0.807|5| 25$ \\
\hline miR-I260b & 0.17907235 & $U_{p}$ & 0.14017212 & 0.75376261 \\
\hline miR-3।98 & 0.17929237 & $U_{p}$ & 0.02123337 & 0.53734625 \\
\hline miR- 1290 & 0.18406083 & $U_{p}$ & 0.03967176 & $0.5987 \mid 978$ \\
\hline miR-4485-3p & 0.1846673 & $U_{p}$ & 0.07340574 & 0.68406327 \\
\hline$m i R-6503-3 p$ & 0.18979698 & Up & 0.28804023 & 0.83439474 \\
\hline miR-582-5p & 0.19045558 & $U_{p}$ & 0.62677845 & $0.949834 \mid$ \\
\hline miR-3940-5p & 0.19057902 & Up & 0.08648205 & 0.70907074 \\
\hline miR-6879-5p & 0.19101714 & $U_{p}$ & 0.02231673 & 0.53734625 \\
\hline miR-6I25 & 0.19106394 & $U_{p}$ & 0.1196758 & 0.74226488 \\
\hline miR-3162-3p & 0.19525376 & $U_{p}$ & 0.06057967 & 0.64832335 \\
\hline miR-4442 & 0.19537199 & $U_{p}$ & 0.02357392 & 0.54093726 \\
\hline$m i R-4669$ & 0.19873977 & $U_{p}$ & 0.05792698 & 0.64832335 \\
\hline miR-8063 & 0.19905207 & Up & 0.33357986 & 0.85489649 \\
\hline miRNABrightCorner30 & 0.20171928 & Up & 0.3490045 & 0.8588192 \\
\hline miR-378d & $0.207 \mid 5078$ & Up & 0.16934925 & 0.77062416 \\
\hline dmr_285 & 0.20740733 & $U_{p}$ & 0.13348728 & 0.74892227 \\
\hline miR-428I & 0.21053809 & $U_{p}$ & 0.14469165 & 0.75376261 \\
\hline miR-3653-3p & 0.21583612 & $U_{p}$ & 0.20986195 & 0.78263372 \\
\hline dmr_3la & 0.21728525 & Up & 0.18051196 & 0.77062416 \\
\hline miR-6800-5p & 0.21733255 & Up & 0.1072548 & 0.74226488 \\
\hline $\operatorname{miR}-8485$ & 0.21842128 & Up & 0.13247253 & 0.74892227 \\
\hline miR-532-3p & 0.22031918 & Up & 0.17750992 & 0.77062416 \\
\hline miR-29b-I-5p & 0.22084105 & $U_{p}$ & 0.22324885 & $0.7910224 \mid$ \\
\hline miR-6069 & 0.22136576 & $U_{p}$ & $0.004|324|$ & 0.34045223 \\
\hline
\end{tabular}

(Continued) 
Table 9 (Continued).

\begin{tabular}{|c|c|c|c|c|}
\hline miRNAs & Log2FoldChange & Regulation & P-value & P-adj \\
\hline miR-574-5p & 0.22143706 & Up & $0.09257 \mid 17$ & $0.7228707 \mid$ \\
\hline miR-4739 & 0.22479676 & Up & 0.46193717 & 0.89396011 \\
\hline miR-7975 & 0.22883797 & Up & 0.21088885 & 0.78263372 \\
\hline miR-472I & 0.2307112 & Up & 0.0365265 & $0.5987 / 978$ \\
\hline miR-1207-5p & 0.23240555 & Up & 0.24200969 & $0.807|5| 25$ \\
\hline miR-4530 & $0.2334301 \mathrm{I}$ & Up & 0.13226628 & 0.74892227 \\
\hline miR-362-5p & 0.24215656 & Up & 0.28398808 & 0.83193599 \\
\hline miR-6767-5p & 0.24557824 & Up & 0.00430298 & 0.34045223 \\
\hline miR-5006-5p & $0.2466579 \mid$ & Up & 0.10970319 & 0.74226488 \\
\hline miR-940 & 0.24920107 & Up & 0.1206625 & 0.74226488 \\
\hline miR-45I2 & 0.24979984 & Up & 0.00061516 & 0.15302397 \\
\hline miR-5I00 & 0.25235575 & Up & 0.34779581 & 0.8588192 \\
\hline miR-6740-5p & 0.25563327 & Up & 0.00409229 & 0.34045223 \\
\hline miR-6088 & 0.25633403 & Up & 0.04560842 & $0.614|250|$ \\
\hline miR-365I & $0.2565736 \mid$ & Up & 0.09007374 & 0.71668583 \\
\hline miR-4428 & 0.26039231 & Up & 0.01098536 & 0.43937422 \\
\hline$m i R-619-5 p$ & 0.26233591 & Up & $0.0289064 \mid$ & 0.59616058 \\
\hline miR-4299 & 0.26378168 & Up & 0.16313713 & 0.76802692 \\
\hline miR-I268a & 0.26900448 & Up & 0.02969275 & 0.59616058 \\
\hline miR-4763-3p & $0.274 \mid I 287$ & Up & 0.15236304 & 0.75393664 \\
\hline miR-4728-5p & 0.27540238 & Up & 0.00902596 & 0.40696367 \\
\hline miR-I273g-3p & $0.2840 \mid 482$ & Up & 0.00853108 & 0.40696367 \\
\hline miR-6780b-5p & $0.2844595 I$ & Up & 0.00201749 & 0.32405862 \\
\hline miR-193a-3p & 0.29191057 & $U_{p}$ & 0.22860566 & $0.79|0224|$ \\
\hline miR-4697-5p & 0.29577233 & Up & 0.02066387 & 0.53734625 \\
\hline miR-338-3p & $0.304075 I 5$ & Up & 0.33547234 & 0.85489649 \\
\hline miR-47/3-3p & 0.31190821 & Up & $0.0044|4| I$ & 0.34045223 \\
\hline miR-6I 27 & 0.31227553 & Up & 0.02937864 & 0.59616058 \\
\hline miR-3663-3p & 0.32622686 & Up & 0.17739186 & 0.77062416 \\
\hline miR-197-5p & 0.32730894 & Up & 0.01165849 & 0.43937422 \\
\hline miR-I225-5p & 0.32794342 & Up & 0.04237249 & 0.60118096 \\
\hline miR-4667-5p & $0.330947 / 3$ & Up & 0.11216581 & 0.74226488 \\
\hline miR-6089 & 0.33321123 & Up & 0.06419436 & 0.65598378 \\
\hline miR-642a-3p & 0.33705253 & Up & $0.035465 I$ & $0.5987 / 978$ \\
\hline miR-3960 & 0.34205792 & Up & 0.21934296 & 0.78840757 \\
\hline miR-6087 & 0.34742973 & Up & 0.01824337 & 0.53734625 \\
\hline miR-4286 & 0.35393924 & Up & 0.15460101 & 0.75393664 \\
\hline miR-6875-5p & 0.35408709 & Up & 0.00825659 & 0.40696367 \\
\hline miR-664b-5p & 0.35527207 & Up & 0.00544933 & 0.36854655 \\
\hline miR-5I3a-5p & 0.37891789 & Up & 0.00367033 & 0.34045223 \\
\hline miR-4459 & 0.38291314 & Up & $0.036 \mid 3368$ & $0.5987 / 978$ \\
\hline$m i R-424-5 p$ & 0.39379877 & Up & $0.176 \mid 3337$ & 0.77062416 \\
\hline miR-6090 & 0.40130574 & Up & $0.1|48| 495$ & 0.74226488 \\
\hline$m i R-365 a-3 p$ & 0.40351876 & Up & 0.01344372 & 0.46067162 \\
\hline miR-I246 & 0.4191497 & Up & $0.00807 \mid I$ & 0.40696367 \\
\hline miR-6785-5p & 0.43652493 & Up & 0.02101025 & 0.53734625 \\
\hline miR-6869-5p & 0.44434072 & Up & 0.00970313 & 0.42994906 \\
\hline miR-34a-5p & 0.45746932 & Up & 0.20090282 & 0.7799749 I \\
\hline miR-6I32 & 0.46392014 & Up & 0.02511952 & 0.55568816 \\
\hline $\mathrm{miR}-45 \mathrm{Ia}$ & 0.5157088 & Up & 0.15065058 & 0.75393664 \\
\hline
\end{tabular}

(Continued) 
Table 9 (Continued).

\begin{tabular}{|c|c|c|c|c|}
\hline miRNAs & Log2FoldChange & Regulation & P-value & P-adj \\
\hline miR-494-3p & $0.52730|7|$ & Up & 0.00892038 & 0.40696367 \\
\hline miR-2I-3p & 0.55990013 & $U_{p}$ & 0.21932847 & 0.78840757 \\
\hline miR-764I & $0.6099726 I$ & $U_{p}$ & $5.26 \times 10^{-5}$ & 0.06753368 \\
\hline miR-378a-3p & 0.65466586 & $U_{p}$ & 0.07470725 & 0.68406327 \\
\hline miR-378i & 0.67902917 & $U_{p}$ & 0.06336176 & 0.65539395 \\
\hline$m i R-3 \mid 30-5 p$ & 0.7469237 & $U_{p}$ & 0.31234802 & $0.8475 \mathrm{I} 25$ \\
\hline $\operatorname{miR}-4516$ & 0.75421011 & $U_{p}$ & 0.12234989 & 0.74454632 \\
\hline miR-6769b-5p & 1.16196437 & Up & 0.00062927 & 0.15302397 \\
\hline
\end{tabular}

only related to M1 macrophage activation induced exacerbation of inflammatory responses but also related to the absolute insulin deficiency caused by pancreatic $\beta$-cells destruction. This study only studies the former, and the effect of these differentially expressed genes on the latter needs further studies.

\section{Conclusions}

In conclusion, we identified differentially expressed circRNAs, lncRNAs, and mRNAs in the PBMCs from T1DM patients and healthy patients and established a T1DM-related circRNA-lncRNA-miRNA-mRNA ceRNA regulatory network for the first time. The construction of

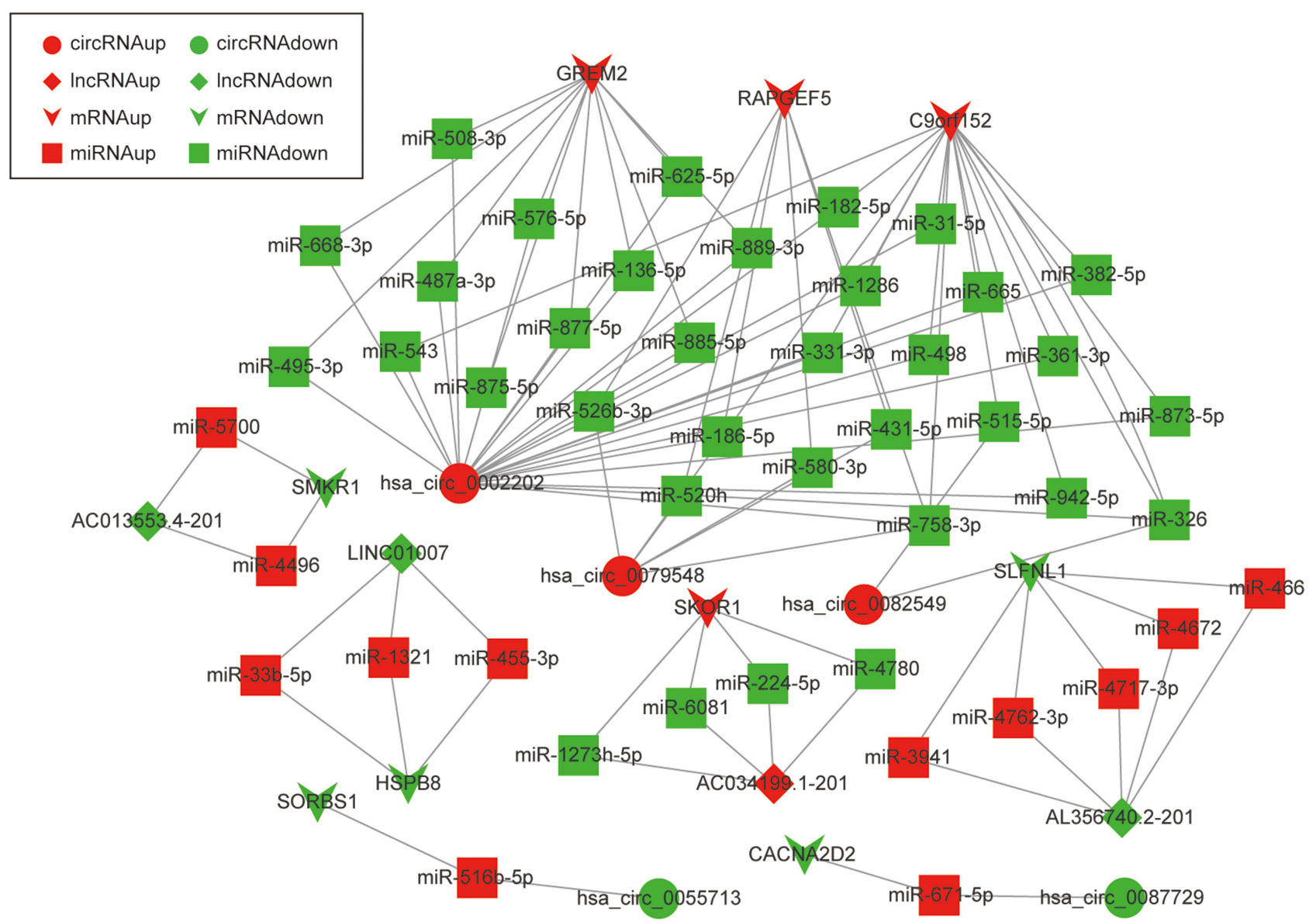

Figure 6 The circRNA-IncRNA-miRNA-mRNA network. The round nodes represent DEcircRNAs, the rhombic nodes represent DElncRNAs, the v-type nodes represent DEmRNAs, the square nodes represent DEmiRNAs. The up or down-regulated genes are represented in red and green, respectively. 

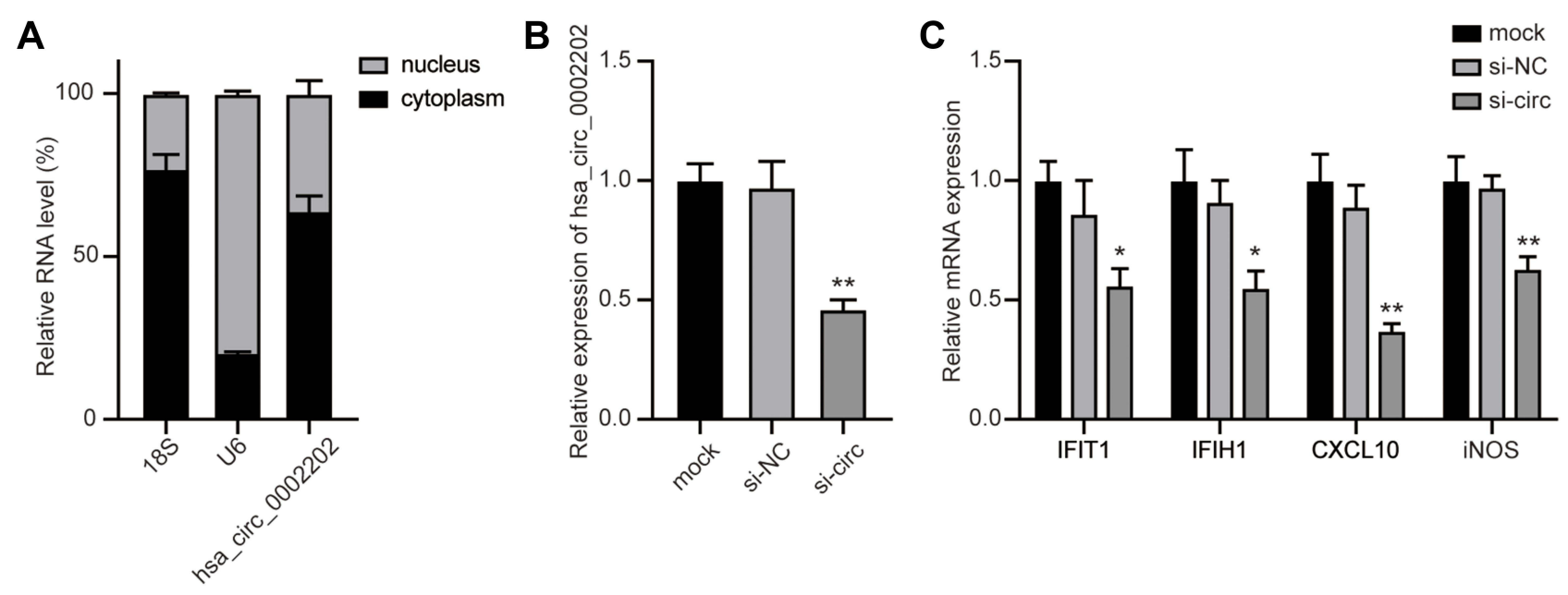

Figure 7 Inhibition of hsa_circ_0002202 suppressed IFN-I-induced inflammation. (A) Subcellular distribution of hsa_circ_0002202 in the nucleus and cytoplasm in THP-Iderived macrophages. (B) qRT-PCR detection of hsa_circ_0002202 expression after transfection with si-NC or si-circ. (C) qRT-PCR detection of IFITI, IFIHI, CXCLI0, and iNOS expression under IFN-I stimulation after treatment with hsa_circ_0002202 siRNA. Data are presented as the mean \pm SD of three experiments and analyzed using one-way ANOVA with Tukey's post-test. $* P<0.05, * * P<0.01$, compared with the si-NC group.

the ceRNA network can help to further analyze the interaction between ncRNAs and mRNAs and provide new insights into the molecular mechanisms in T1DM. The new ceRNA network in this study will contribute to the diagnosis and treatment of T1DM. Of course, our research findings are only the first step. In the follow-up experimental verification study, we will rely on multiple levels, including clinical tissue samples, cell line studies, and animal models, to analyze the internal function mechanism.

\section{Abbreviations}

$\mathrm{BP}$, biological processes; CAP, c-Cbl-associated protein; CC, cellular components; circRNA, circular RNA; ceRNA, competitive endogenous RNA; DEcircRNAs, differentially expressed circRNAs; DElncRNAs, differentially expressed lncRNAs; DEGs, differentially expressed genes; DEmRNAs, differentially expressed mRNAs; ECM, extracellular matrix components; GEO, Gene Expression Omnibus; GO, Gene Ontology; IFN-I, type I interferon; KEGG, Kyoto Encyclopedia of Genes and Genomes; lncRNA, long non-coding RNA; miRNA, microRNA; MRE, miRNA response element; MF, molecular functions; ncRNA, non-coding RNA; PBMCs, peripheral blood mononuclear cells; PMA, phorbol 12myristate 13-acetate; qRT-PCR, Quantitative real-time PCR; T1DM, Type 1 diabetes mellitus.

\section{Author Contributions}

Both authors made a significant contribution to the work reported, whether that is in the conception, study design, execution, acquisition of data, analysis and interpretation, or in all these areas; took part in drafting, revising or critically reviewing the article; gave final approval of the version to be published; have agreed on the journal to which the article has been submitted; and agree to be accountable for all aspects of the work.

\section{Funding}

There is no funding to report.

\section{Disclosure}

The authors declare that they have no conflicts of interest for this work.

\section{References}

1. Pozzilli P, Maddaloni E, Buzzetti R. Combination immunotherapies for type 1 diabetes mellitus. Nat Rev Endocrinol. 2015;11(5):289-297. doi: $10.1038 /$ nrendo.2015.8

2. Ni Q, Pham NB, Meng WS, Zhu G, Chen X. Advances in immunotherapy of type I diabetes. Adv Drug Deliv Rev. 2019;139:83-91. doi:10.1016/j.addr.2018.12.003

3. Cerna M. Epigenetic Regulation in Etiology of Type 1 Diabetes Mellitus. Int J Mol Sci. 2019;21(1):254. doi:10.3390/ijms21010036

4. Gastol J, Kapusta P, Polus A, et al. Epigenetic mechanism in search for the pathomechanism of diabetic neuropathy development in diabetes mellitus type 1 (T1DM). Endocrine. 2020;68(1):235-240. doi:10.1007/s12020-019-02172-9

5. Ilonen J, Lempainen J, Veijola R. The heterogeneous pathogenesis of type 1 diabetes mellitus. Nat Rev Endocrinol. 2019;15(11):635-650. doi:10.1038/s41574-019-0254-y

6. Paschou SA, Papadopoulou-Marketou N, Chrousos GP, KanakaGantenbein C. On type 1 diabetes mellitus pathogenesis. Endocr Connect. 2018;7(1):R38-R46. doi:10.1530/EC-17-0347 
7. Diaz-Valencia PA, Bougneres P, Valleron AJ. Global epidemiology of type 1 diabetes in young adults and adults: a systematic review. $B M C$ Public Health. 2015;15:255. doi:10.1186/s12889-015-1591-y

8. Thomas NJ, Jones SE, Weedon MN, Shields BM, Oram RA, Hattersley AT. Frequency and phenotype of type 1 diabetes in the first six decades of life: a cross-sectional, genetically stratified survival analysis from UK Biobank. Lancet Diabetes Endocrinol. 2018;6 (2):122-129. doi:10.1016/S2213-8587(17)30362-5

9. Chiang JL, Kirkman MS, Laffel LM, Peters AL. Type 1 Diabetes Sourcebook A. Type 1 diabetes through the life span: a position statement of the American Diabetes Association. Diabetes Care. 2014;37(7):2034-2054. doi:10.2337/dc14-1140

10. Patterson CC, Karuranga S, Salpea P, et al. Worldwide estimates of incidence, prevalence and mortality of type 1 diabetes in children and adolescents: results from the International Diabetes Federation Diabetes Atlas, 9th edition. Diabetes Res Clin Pract. 2019;157:107842. doi:10.1016/j.diabres.2019.107842

11. Liu J, Ren ZH, Qiang H, et al. Trends in the incidence of diabetes mellitus: results from the Global Burden of Disease Study 2017 and implications for diabetes mellitus prevention. BMC Public Health. 2020;20(1):1415. doi:10.1186/s12889-020-09502-x

12. Tao B, Pietropaolo M, Atkinson M, Schatz D, Taylor D. Estimating the cost of type 1 diabetes in the U.S.: a propensity score matching method. PLoS One. 2010;5(7):e11501. doi:10.1371/journal.pone.0011501

13. Lapolla A, Amaro F, Bruttomesso D, et al. Diabetic ketoacidosis: a consensus statement of the Italian Association of Medical Diabetologists (AMD), Italian Society of Diabetology (SID), Italian Society of Endocrinology and Pediatric Diabetoloy (SIEDP). Nutr Metab Cardiovasc Dis. 2020;30(10):1633-1644. doi:10.1016/j. numecd.2020.06.006

14. Williams SM, Eleftheriadou A, Alam U, Cuthbertson DJ, Jph W. Cardiac Autonomic Neuropathy in Obesity, the Metabolic Syndrome and Prediabetes: a Narrative Review. Diabetes Ther. 2019;10 (6):1995-2021. doi:10.1007/s13300-019-00693-0

15. Sousa GR, Pober D, Galderisi A, et al. Glycemic Control, Cardiac Autoimmunity, and Long-Term Risk of Cardiovascular Disease in Type 1 Diabetes Mellitus. Circulation. 2019;139(6):730-743. doi:10.1161/CIRCULATIONAHA.118.036068

16. Gillard P, Schnell O, Groop PH. The nephrological perspective on SGLT-2 inhibitors in type 1 diabetes. Diabetes Res Clin Pract. 2020;170:108462. doi:10.1016/j.diabres.2020.108462

17. Skyler JS, Bakris GL, Bonifacio E, et al. Differentiation of Diabetes by Pathophysiology, Natural History, and Prognosis. Diabetes. 2017;66(2):241-255. doi:10.2337/db16-0806

18. Crossen S, Xing G, Hoch JS. Changing costs of type 1 diabetes care among US children and adolescents. Pediatr Diabetes. 2020;21 (4):644-648. doi:10.1111/pedi.12996

19. Zou Q, Qu K, Luo Y, Yin D, Ju Y, Tang H. Predicting Diabetes Mellitus With Machine Learning Techniques. Front Genet. 2018;9:515. doi:10.3389/fgene.2018.00515

20. Taheri M, Eghtedarian R, Dinger ME, Ghafouri-Fard S. Emerging roles of non-coding RNAs in the pathogenesis of type 1 diabetes mellitus. Biomed Pharmacother. 2020;129:110509. doi:10.1016/j. biopha.2020.110509

21. Geng G, Zhang Z, Cheng L. Identification of a Multi-Long Noncoding RNA Signature for the Diagnosis of Type 1 Diabetes Mellitus. Front Bioeng Biotechnol. 2020;8:553. doi:10.3389/fbioe.2020.00553

22. Li Y, Zhou Y, Zhao M, et al. Differential Profile of Plasma Circular RNAs in Type 1 Diabetes Mellitus. Diabetes Metab J. 2020;44 (6):854-865. doi:10.4093/dmj.2019.0151

23. Gonzalez-Moro I, Olazagoitia-Garmendia A, Colli ML, et al. The T1D-associated lncRNA Lnc13 modulates human pancreatic beta cell inflammation by allele-specific stabilization of STAT1 mRNA. Proc Natl Acad Sci U S A. 2020;117(16):9022-9031. doi:10.1073/ pnas. 1914353117
24. Ding H, Wang F, Shi $\mathrm{X}$, et al. LncRNA MALAT1 induces the dysfunction of beta cells via reducing the histone acetylation of the PDX-1 promoter in type 1 diabetes. Exp Mol Pathol. 2020;114:104432. doi:10.1016/j.yexmp.2020.104432

25. Zhang C, Han X, Yang L, et al. Circular RNA circPPM1F modulates M1 macrophage activation and pancreatic islet inflammation in type 1 diabetes mellitus. Theranostics. 2020;10(24):10908-10924. doi:10.7150/ thno. 48264

26. Hansen TB, Jensen TI, Clausen BH, et al. Natural RNA circles function as efficient microRNA sponges. Nature. 2013;495 (7441):384-388. doi:10.1038/nature11993

27. Khorkova O, Hsiao J, Wahlestedt C. Basic biology and therapeutic implications of lncRNA. Adv Drug Deliv Rev. 2015;87:15-24. doi:10.1016/j.addr.2015.05.012

28. Salmena L, Poliseno L, Tay Y, Kats L, Pandolfi PP. A ceRNA hypothesis: the Rosetta Stone of a hidden RNA language? Cell. 2011;146(3):353-358. doi:10.1016/j.cell.2011.07.014

29. Yang F, Chen Y, Xue Z, et al. High-Throughput Sequencing and Exploration of the IncRNA-circRNA-miRNA-mRNA Network in Type 2 Diabetes Mellitus. Biomed Res Int. 2020;2020:8162524. doi: $10.1155 / 2020 / 8162524$

30. Hu W, Ding Y, Wang S, Xu L, Yu H. The Construction and Analysis of the Aberrant lncRNA-miRNA-mRNA Network in Adipose Tissue from Type 2 Diabetes Individuals with Obesity. J Diabetes Res. 2020;2020:3980742. doi:10.1155/2020/3980742

31. Lin Z, Li X, Zhan X, et al. Construction of competitive endogenous RNA network reveals regulatory role of long non-coding RNAs in type 2 diabetes mellitus. J Cell Mol Med. 2017;21(12):3204-3213. doi: $10.1111 / \mathrm{jcmm} .13224$

32. Kong X, Liu CX, Wang GD, et al. LncRNA LEGLTBC Functions as a ceRNA to Antagonize the Effects of miR-34a on the Downregulation of SIRT1 in Glucolipotoxicity-Induced INS-1 Beta Cell Oxidative Stress and Apoptosis. Oxid Med Cell Longev. 2019;2019:4010764. doi:10.1155/2019/4010764

33. Luo S, Deng M, Xie Z, Li X, Huang G, Zhou Z. Circulating circular RNAs profiles associated with type 1 diabetes. Diabetes Metab Res Rev. 2021;37(3):e3394. doi:10.1002/dmrr.3394

34. Shi Q, Yao H. Signature RNAS and related regulatory roles in type 1 diabetes mellitus based on competing endogenous RNA regulatory network analysis. BMC Med Genomics. 2021;14(1):133. doi:10.1186/ s12920-021-00931-0

35. Edgar R, Domrachev M, Lash AE. Gene Expression Omnibus: NCBI gene expression and hybridization array data repository. Nucleic Acids Res. 2002;30(1):207-210. doi:10.1093/nar/30.1.207

36. Yang L, Han X, Zhang C, et al. Hsa_circ_0060450 Negatively Regulates Type I Interferon-Induced Inflammation by Serving as miR-199a-5p Sponge in Type 1 Diabetes Mellitus. Front Immunol. 2020;11:576903. doi:10.3389/fimmu.2020.576903

37. Gautier L, Cope L, Bolstad BM, Irizarry RA. affy-analysis of Affymetrix GeneChip data at the probe level. Bioinformatics. 2004;20(3):307-315. doi:10.1093/bioinformatics/btg405

38. Ritchie ME, Phipson B, Wu D, et al. limma powers differential expression analyses for RNA-sequencing and microarray studies. Nucleic Acids Res. 2015;43(7):e47. doi:10.1093/nar/gkv007

39. Yu G, Wang LG, Han Y, He QY. clusterProfiler: an R package for comparing biological themes among gene clusters. OMICS. 2012;16 (5):284-287. doi:10.1089/omi.2011.0118

40. Ashburner M, Ball CA, Blake JA, et al. Gene ontology: tool for the unification of biology. The Gene Ontology Consortium. Nat Genet. 2000;25(1):25-29. doi:10.1038/75556

41. Kanehisa M, Goto S, Furumichi M, Tanabe M, Hirakawa M. KEGG for representation and analysis of molecular networks involving diseases and drugs. Nucleic Acids Res. 2010;38(Database issue):D355360. doi:10.1093/nar/gkp896 
42. Shannon P, Markiel A, Ozier O, et al. Cytoscape: a software environment for integrated models of biomolecular interaction networks. Genome Res. 2003;13(11):2498-2504. doi:10.1101/gr.1239303

43. Vandesompele J, De Preter K, Pattyn F, et al. Accurate normalization of real-time quantitative RT-PCR data by geometric averaging of multiple internal control genes. Genome Biol. 2002;3(7): RESEARCH0034. doi:10.1186/gb-2002-3-7-research0034

44. Andersen CL, Jensen JL, Orntoft TF. Normalization of real-time quantitative reverse transcription-PCR data: a model-based variance estimation approach to identify genes suited for normalization, applied to bladder and colon cancer data sets. Cancer Res. 2004;64 (15):5245-5250. doi:10.1158/0008-5472.CAN-04-0496

45. Zhou M, Gao M, Luo Y, Gui R, Ji H. Long non-coding RNA metallothionein 1 pseudogene 3 promotes p2y12 expression by sponging miR-126 to activate platelet in diabetic animal model. Platelets. 2019;30(4):452-459. doi:10.1080/09537104.2018.1457781

46. Chen W, Peng R, Sun Y, et al. The topological key lncRNA H2k2 from the ceRNA network promotes mesangial cell proliferation in diabetic nephropathy via the miR-449a/b/Trim11/Mek signaling pathway. FASEB J. 2019;33(10):11492-11506. doi:10.1096/fj.201900522R

47. Zhou B, Yu JW. A novel identified circular RNA, circRNA 010567 promotes myocardial fibrosis via suppressing miR-141 by targeting TGF-beta1. Biochem Biophys Res Commun. 2017;487(4):769-775. doi:10.1016/j.bbrc.2017.04.044

48. Li C, Wei B, Zhao J. Competing endogenous RNA network analysis explores the key lncRNAs, miRNAs, and mRNAs in type 1 diabetes. BMC Med Genomics. 2021;14(1):35. doi:10.1186/s12920-021-00877-3

49. Xie C, Chen B, Wu B, Guo J, Shi Y, Cao Y. CircSAMD4A regulates cell progression and epithelialmesenchymal transition by sponging miR3423p via the regulation of FZD7 expression in osteosarcoma. Int J Mol Med. 2020;46(1):107-118. doi:10.3892/ijmm.2020.4585

50. Liu Y, Liu H, Li Y, et al. Circular RNA SAMD4A controls adipogenesis in obesity through the miR-138-5p/EZH2 axis. Theranostics. 2020;10(10):4705-4719. doi:10.7150/thno.42417

51. Hu X, Ma R, Cao J, Du X, Cai X, Fan Y. CircSAMD4A aggravates H/Rinduced cardiomyocyte apoptosis and inflammatory response by sponging miR-138-5p. J Cell Mol Med. 2020. doi:10.1111/jcmm.16093

52. Zurawek M, Dzikiewicz-Krawczyk A, Izykowska K, et al. miR-487a$3 \mathrm{p}$ upregulated in type 1 diabetes targets CTLA4 and FOXO3. Diabetes Res Clin Pract. 2018;142:146-153. doi:10.1016/j. diabres.2018.05.044

53. Azhir Z, Dehghanian F, Hojati Z. Increased expression of microRNAs, miR-20a and miR-326 in PBMCs of patients with type 1 diabetes. Mol Biol Rep. 2018;45(6):1973-1980. doi:10.1007/ s11033-018-4352-z

54. Sebastiani G, Grieco FA, Spagnuolo I, Galleri L, Cataldo D, Dotta F. Increased expression of microRNA miR-326 in type 1 diabetic patients with ongoing islet autoimmunity. Diabetes Metab Res Rev. 2011;27(8):862-866. doi:10.1002/dmrr. 1262

55. Garcia-Diaz DF, Pizarro C, Camacho-Guillen P, Codner E, Soto N, Perez-Bravo F. Expression of miR-155, miR-146a, and miR-326 in T1D patients from Chile: relationship with autoimmunity and inflammatory markers. Arch Endocrinol Metab. 2018;62(1):34-40. doi:10.20945/2359-3997000000006
56. Wischer JL, Oermann MH, Zadvinskis IM, Kinney KC. Effects of iPad Video Education on Patient Knowledge, Satisfaction, and Cardiac Rehabilitation Attendance. Qual Manag Health Care. 2018;27(4):204-208. doi:10.1097/QMH.0000000000000185

57. Li M, Zhang S, Qiu Y, et al. Upregulation of miR-665 promotes apoptosis and colitis in inflammatory bowel disease by repressing the endoplasmic reticulum stress components XBP1 and ORMDL3. Cell Death Dis. 2017;8(3):e2699. doi:10.1038/cddis.2017.76

58. Wang G, Yuan J, Cai X, et al. HucMSC-exosomes carrying miR-326 inhibit neddylation to relieve inflammatory bowel disease in mice. Clin Transl Med. 2020;10(2):e113. doi:10.1002/ctm2.113

59. Olaru AV, Yamanaka S, Vazquez C, et al. MicroRNA-224 negatively regulates p21 expression during late neoplastic progression in inflammatory bowel disease. Inflamm Bowel Dis. 2013;19(3):471-480. doi:10.1097/MIB.0b013e31827e78eb

60. Bogdani M, Korpos E, Simeonovic CJ, Parish CR, Sorokin L, Wight TN. Extracellular matrix components in the pathogenesis of type 1 diabetes. Curr Diab Rep. 2014;14(12):552. doi:10.1007/s11892-0140552-7

61. Marro BS, Legrain S, Ware BC, Oldstone MB. Macrophage IFN-I signaling promotes autoreactive $\mathrm{T}$ cell infiltration into islets in type 1 diabetes model. JCI Insight. 2019;4(2):24. doi:10.1172/jci. insight. 125067

62. Chang TJ, Wang WC, Hsiung CA, et al. Genetic Variation in the Human SORBS1 Gene is Associated With Blood Pressure Regulation and Age at Onset of Hypertension: a SAPPHIRe Cohort Study. Medicine. 2016;95(10):e2970. doi:10.1097/MD.0000000000002970

63. Germain M, Pezzolesi MG, Sandholm N, et al. SORBS1 gene, a new candidate for diabetic nephropathy: results from a multi-stage genome-wide association study in patients with type 1 diabetes. Diabetologia. 2015;58(3):543-548. doi:10.1007/s00125-014-3459-6

64. Fu Y, Zhou N, Bai W, et al. Association of the CACNA2D2 gene with schizophrenia in Chinese Han population. PeerJ. 2020;8:e8521. doi: $10.7717 /$ peerj.8521

65. Huang Q, Deng G, Wei R, Wang Q, Zou D, Wei J. Comprehensive Identification of Key Genes Involved in Development of Diabetes Mellitus-Related Atherogenesis Using Weighted Gene Correlation Network Analysis. Front Cardiovasc Med. 2020;7:580573. doi:10.3389/fcvm.2020.580573

66. Sanders LN, Schoenhard JA, Saleh MA, et al. BMP Antagonist Gremlin 2 Limits Inflammation After Myocardial Infarction. Circ Res. 2016;119(3):434-449. doi:10.1161/CIRCRESAHA.116.308700

67. Bruinsma IB, de Jager M, Carrano A, et al. Small heat shock proteins induce a cerebral inflammatory reaction. $J$ Neurosci. 2011;31 (33):11992-12000. doi:10.1523/JNEUROSCI.0945-11.2011

68. Chen LL. The biogenesis and emerging roles of circular RNAs. Nat Rev Mol Cell Biol. 2016;17(4):205-211. doi:10.1038/nrm.2015.32

69. Engreitz JM, Haines JE, Perez EM, et al. Local regulation of gene expression by lncRNA promoters, transcription and splicing. Nature. 2016;539(7629):452-455. doi:10.1038/nature20149

Diabetes, Metabolic Syndrome and Obesity: Targets and Therapy

Dovepress

\section{Publish your work in this journal}

Diabetes, Metabolic Syndrome and Obesity: Targets and Therapy is an international, peer-reviewed open-access journal committed to the rapid publication of the latest laboratory and clinical findings in the fields of diabetes, metabolic syndrome and obesity research. Original research, review, case reports, hypothesis formation, expert opinion and commentaries are all considered for publication. The manuscript management system is completely online and includes a very quick and fair peer-review system, which is all easy to use. Visit http://www.dovepress.com/testimonials.php to read real quotes from published authors. 